\title{
Chiral Analysis Report
}

Sample Name:

Date Acquired:

Acq. Method Set:

Injection Volume:

Column name:

Co-Solvent:

Gradient (B\%) :

Back Pressure(psi):

Flow ( $\mathrm{ml} / \mathrm{min})$ :

Temperature :

Detector:
ANAL_SFC-PH-CYT-A-1096-0-5(56403-150A1)1T

7/1/2016 12:29:28

B4_7

$2.0 \overline{\mathrm{ul}}$

CHIRALPAK IA-3 100*3mm,3um

$\mathrm{MeOH}(20 \mathrm{mMNH} 3)$

$10 \%$ to $50 \%$ in $4.0 \mathrm{~min}$, hold $2.0 \mathrm{~min}$ at $50 \%$

1500.000

2

35

$220 \mathrm{~nm}$

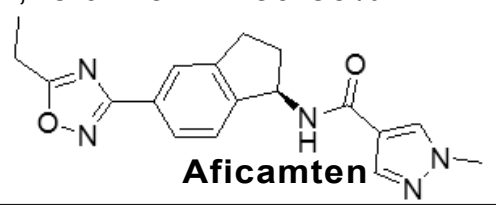

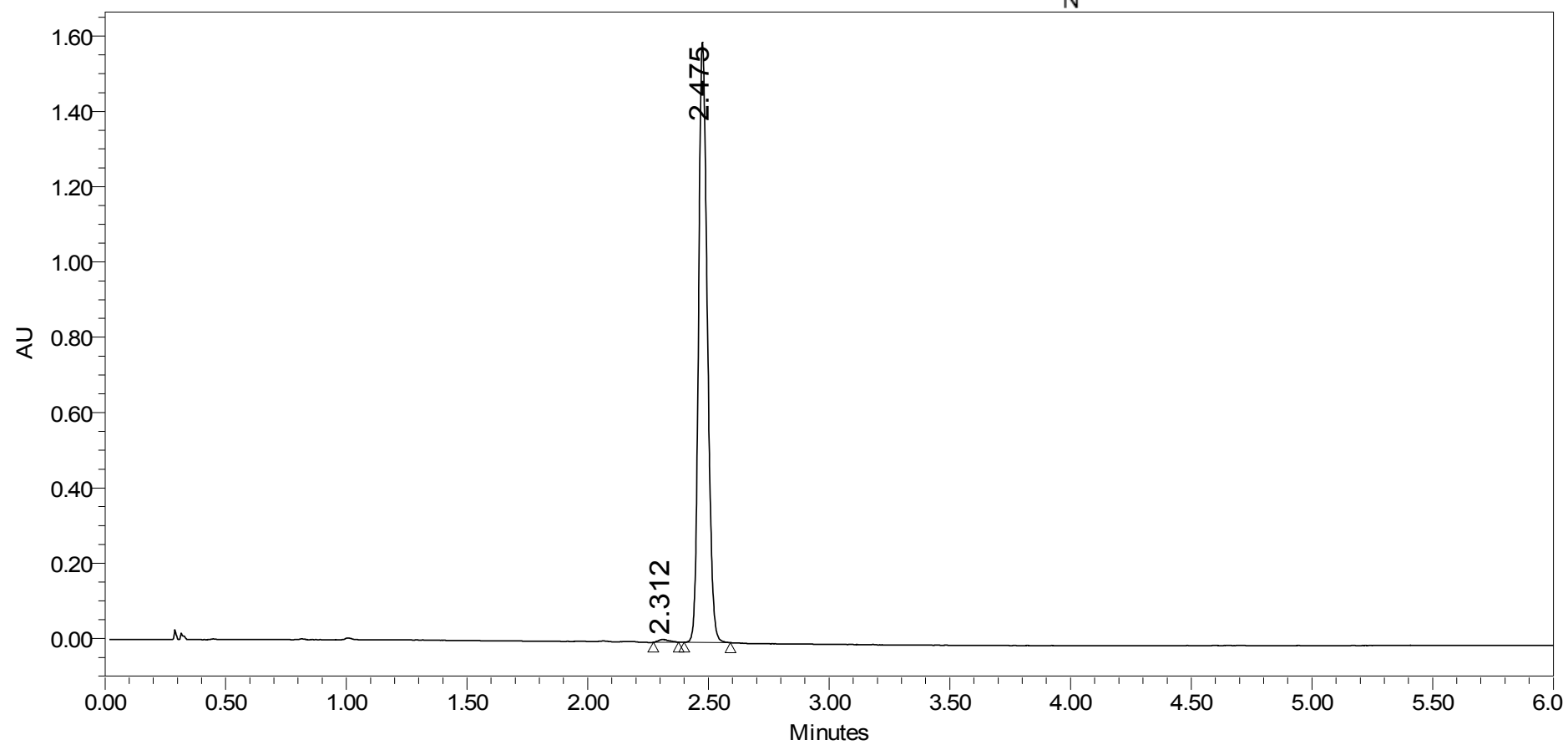

\begin{tabular}{|c|c|r|r|}
\hline & RT & Area & \% Area \\
\hline 1 & 2.312 & 21433 & 0.53 \\
\hline 2 & 2.475 & 4038223 & 99.47 \\
\hline
\end{tabular}

Reported by User: System

Project Name: 2016\201606

Report Method: Gradient

Date Printed:

Report Method II 1455

$7 / 1 / 2016$

Page: 1 of 1

18:12:14 PRC 


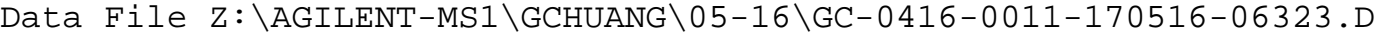

Sample Name: gc-0416-001

Easy-Access Method: '10M_POS'

$========================$
Acq. Operator : chemequip

Sample Operator : chemequip

Acq. Instrument : AGILENTMS1

Injection Date : 5/17/2016 8:34:36 AM

Location : Vial 87

Inj : 1

Method : C : \CHEM32 $2 \backslash$ METHODS $\backslash 10 M \_P O S . M$

Last changed : 5/17/2016 8:33:33 AM by chemequip

(modified after loading)

Sample Info : Easy-Access Method: '10M_Pos'
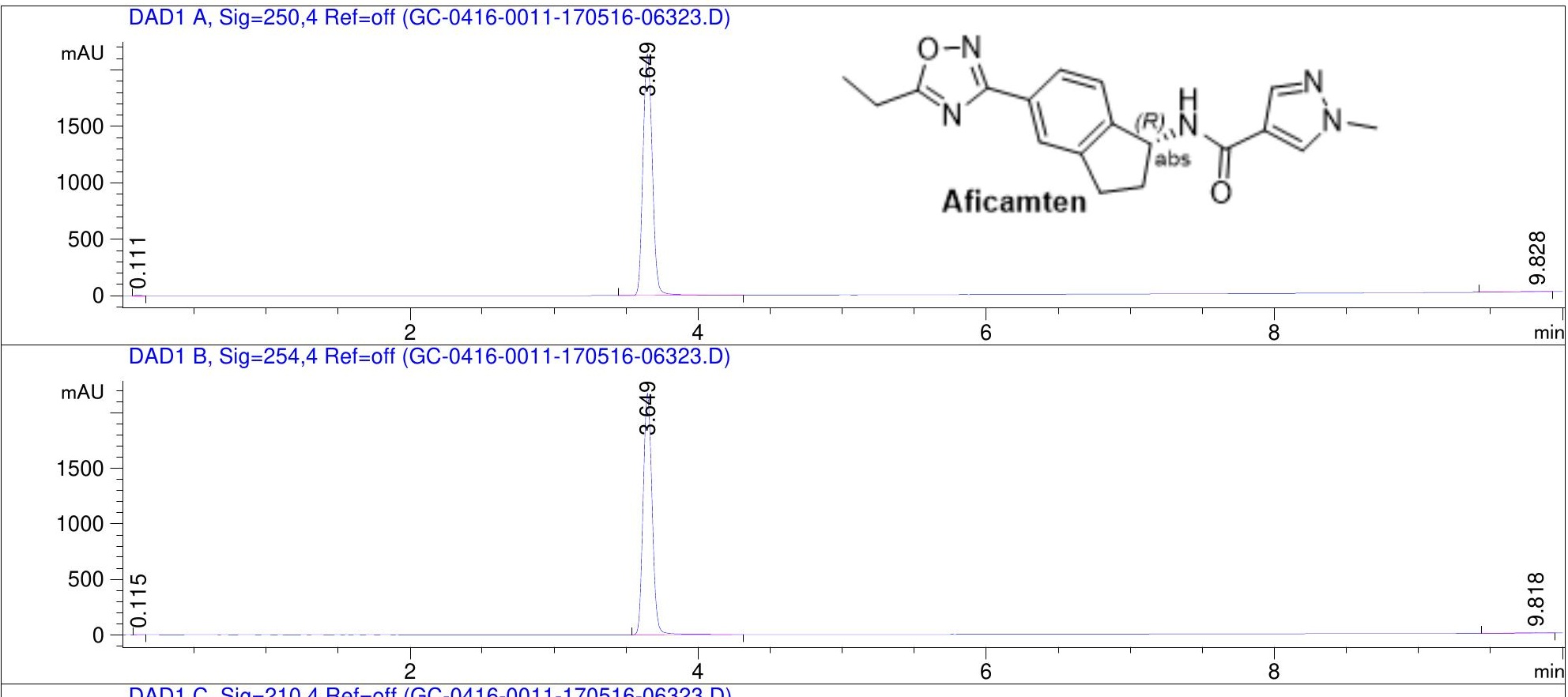

DAD1 C, Sig=210,4 Ref=off (GC-0416-0011-170516-06323.D)
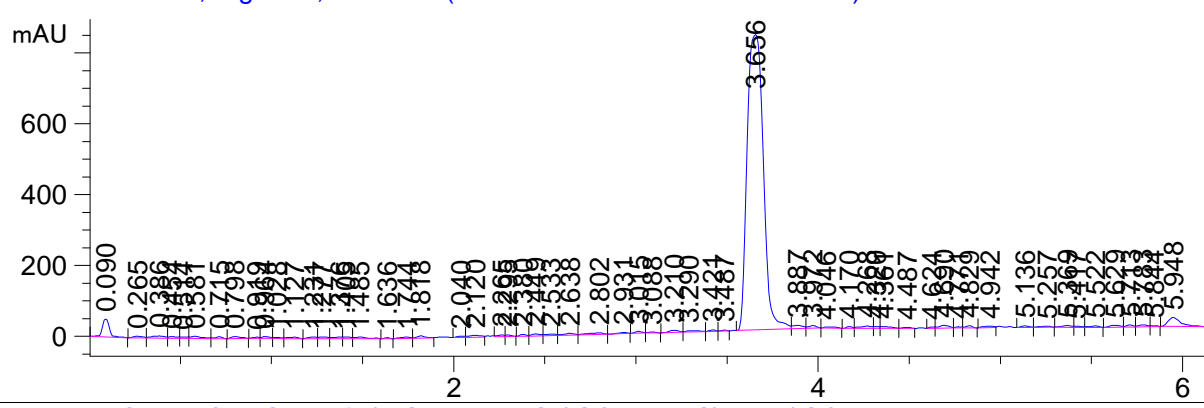

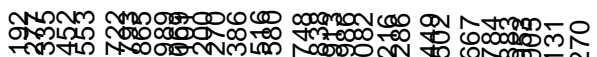

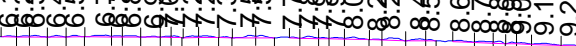

6

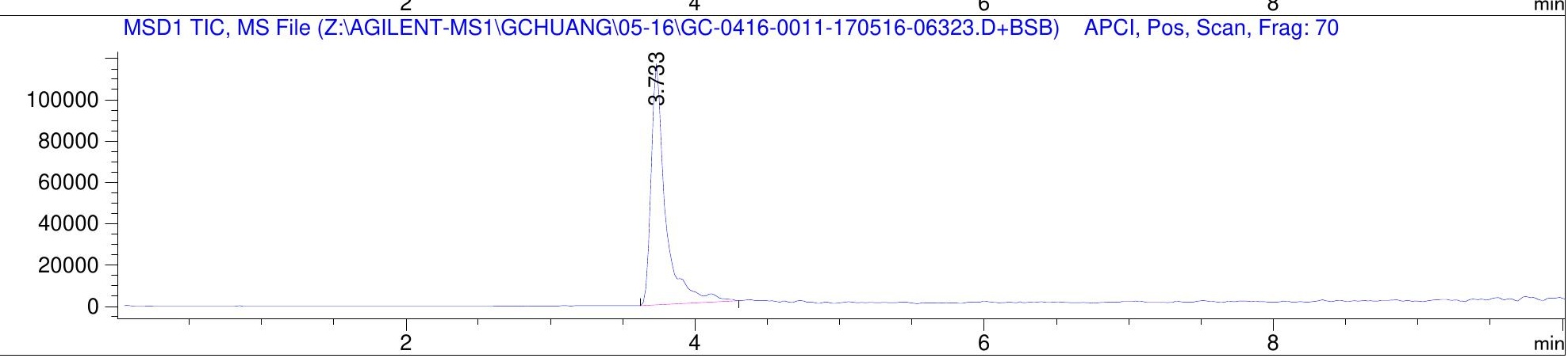




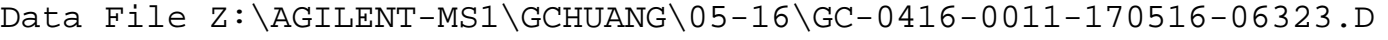

Sample Name: gc-0416-001

MS Signal: MSD1 TIC, MS File, APCI, Pos, Scan, Frag: 70

Subtracted background from 0.000 to $3.000 \mathrm{~min}$.

Spectra averaged over upper half of peaks.

Noise Cutoff: 1000 counts.

Reportable Ion Abundance: > 10\%.

LC Signal: DAD1 A, Sig=250,4 Ref=off

Peak matching window: $0.1 \mathrm{~min}$

$\begin{array}{crrrr}\begin{array}{c}\text { Retention } \\ \text { Time (LC) }\end{array} & \text { LC Area } & \begin{array}{l}\text { Retention } \\ \text { Time (MS) }\end{array} & \text { MS Area } & \text { Mol. Weight } \\ 0.111 & 8 & - & & - \\ 3.649 & 9629 & 3.733 & 807413 & 339.10 \text { I } \\ & & & & 338.15 \text { I } \\ 9.828 & 37 & - & -\end{array}$

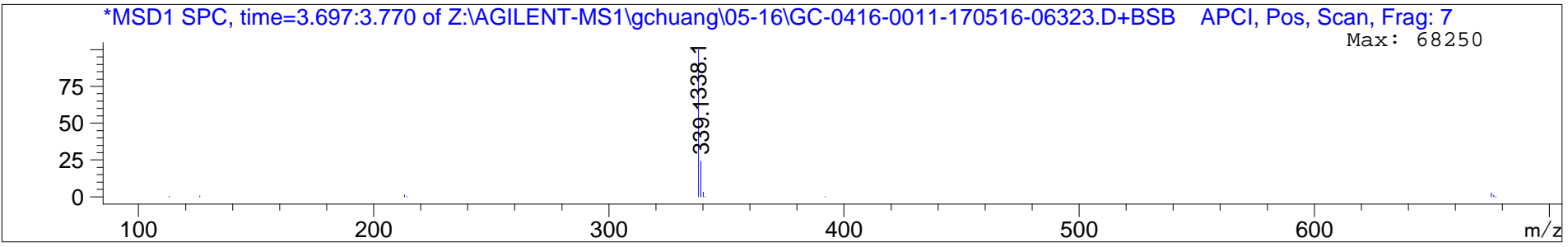




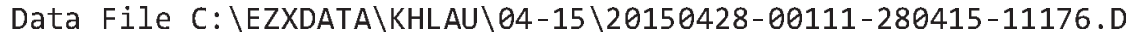

Sample Name: 20150428-0011

Easy-Access Method: '10M_POS'

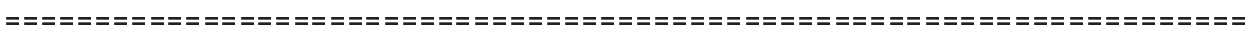

Acq. Operator : chemequip

Sample Operator : chemequip

Acq. Instrument : LCMS 2

Injection Date : 4/28/2015 2:12:50 PM

Location : Vial 32

Inj : 1

Inj Volume : $5.000 \mu \mathrm{I}$

Acq. Method : C: \CHEM32\1\METHODS \10M_POS.M

Last changed : 4/28/2015 2:12:07 PM by chemequip (modified after loading)

Analysis Method : C: $\backslash$ CHEM32\} \backslash 1 \backslash \text { METHODS } \backslash 3 M + P O S . M

Last changed

: 4/29/2015 11:45:32 AM by chemequip (modified after loading)

Sample Info : Easy-Access Method: '10M_POS'
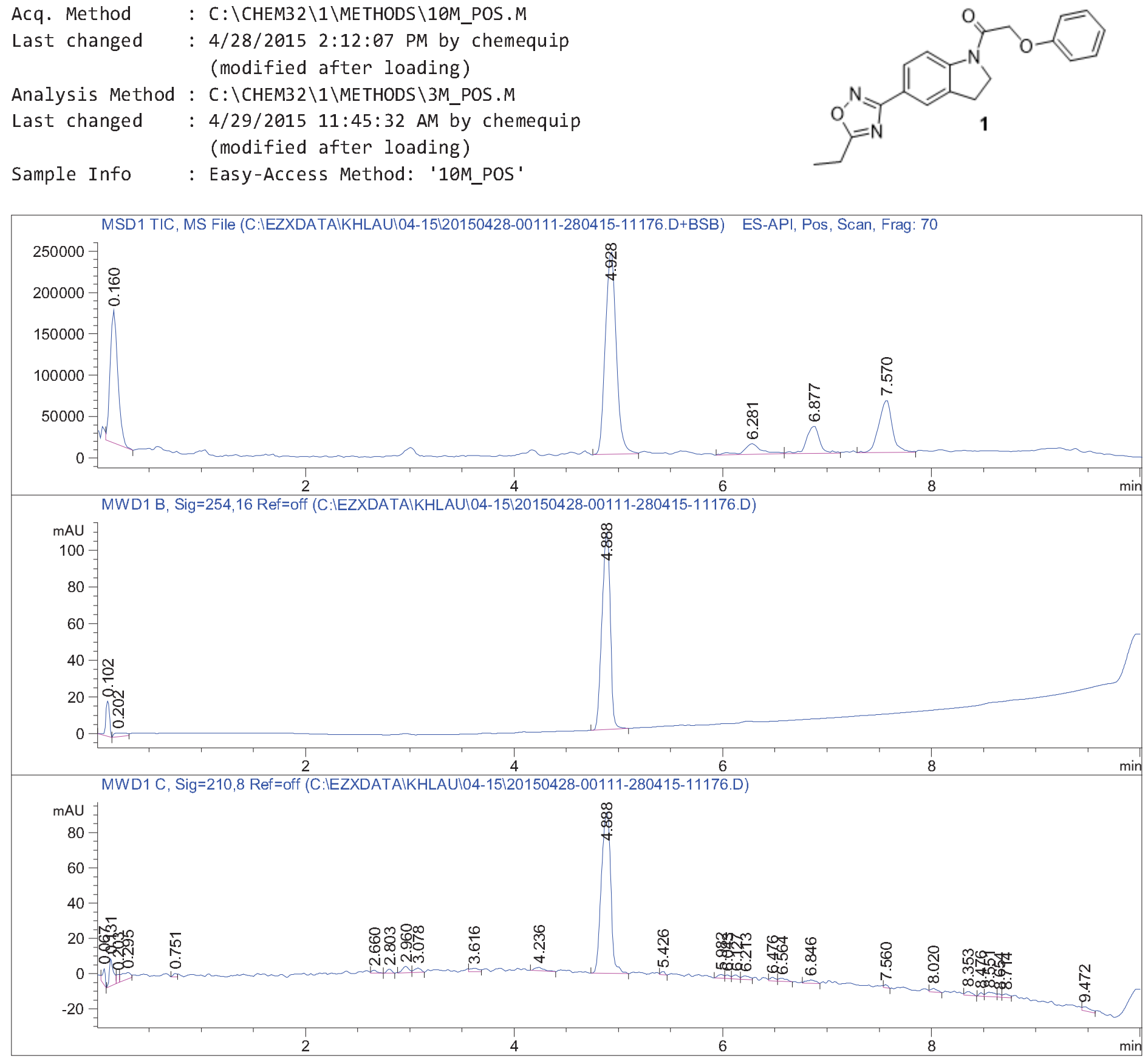
Data File C:\EZXDATA \KHLAU\04-15\20150428-00111-280415-11176.D

Sample Name: 20150428-0011

MS Signal: MSD1 TIC, MS File, ES-API, Pos, Scan, Frag: 70

Subtracted background from 0.000 to $3.000 \mathrm{~min}$.

Spectra averaged over upper half of peaks.

Noise Cutoff: 1000 counts.

Reportable Ion Abundance: >10\%.

LC Signal: MWD1 B, Sig=254, 16 Ref=off

Peak matching window: $0.1 \mathrm{~min}$

\begin{tabular}{|c|c|c|c|c|}
\hline Retention & & Retention & & Mol. Weig \\
\hline Time (LC) & LC Area & Time (MS) & MS Area & or Ion \\
\hline 0.102 & 48 & 0.160 & 820568 & $130.20 \mathrm{I}$ \\
\hline & & & & $100.15 \mathrm{I}$ \\
\hline 0.202 & 15 & - & - & \\
\hline 4.888 & 599 & 4.928 & 1792095 & $351.20 \mathrm{I}$ \\
\hline & & & & $350.20 \mathrm{I}$ \\
\hline - & - & 6.281 & 157304 & $376.10 \mathrm{I}$ \\
\hline & & & & $375.20 \mathrm{I}$ \\
\hline - & - & 6.877 & 287249 & $354.30 \mathrm{I}$ \\
\hline & & & & $353.30 \mathrm{I}$ \\
\hline & & & & $331.30 \mathrm{I}$ \\
\hline & & & & $314.25 \mathrm{I}$ \\
\hline & & & & $313.30 \mathrm{I}$ \\
\hline & & & & $144.95 \mathrm{I}$ \\
\hline - & - & 7.570 & 561285 & $557.60 \mathrm{I}$ \\
\hline & & & & $382.25 \mathrm{I}$ \\
\hline & & & & $381.30 \mathrm{I}$ \\
\hline & & & & $360.35 \mathrm{I}$ \\
\hline & & & & $359.30 \mathrm{I}$ \\
\hline & & & & $342.35 \mathrm{I}$ \\
\hline & & & & $341.30 \mathrm{I}$ \\
\hline
\end{tabular}
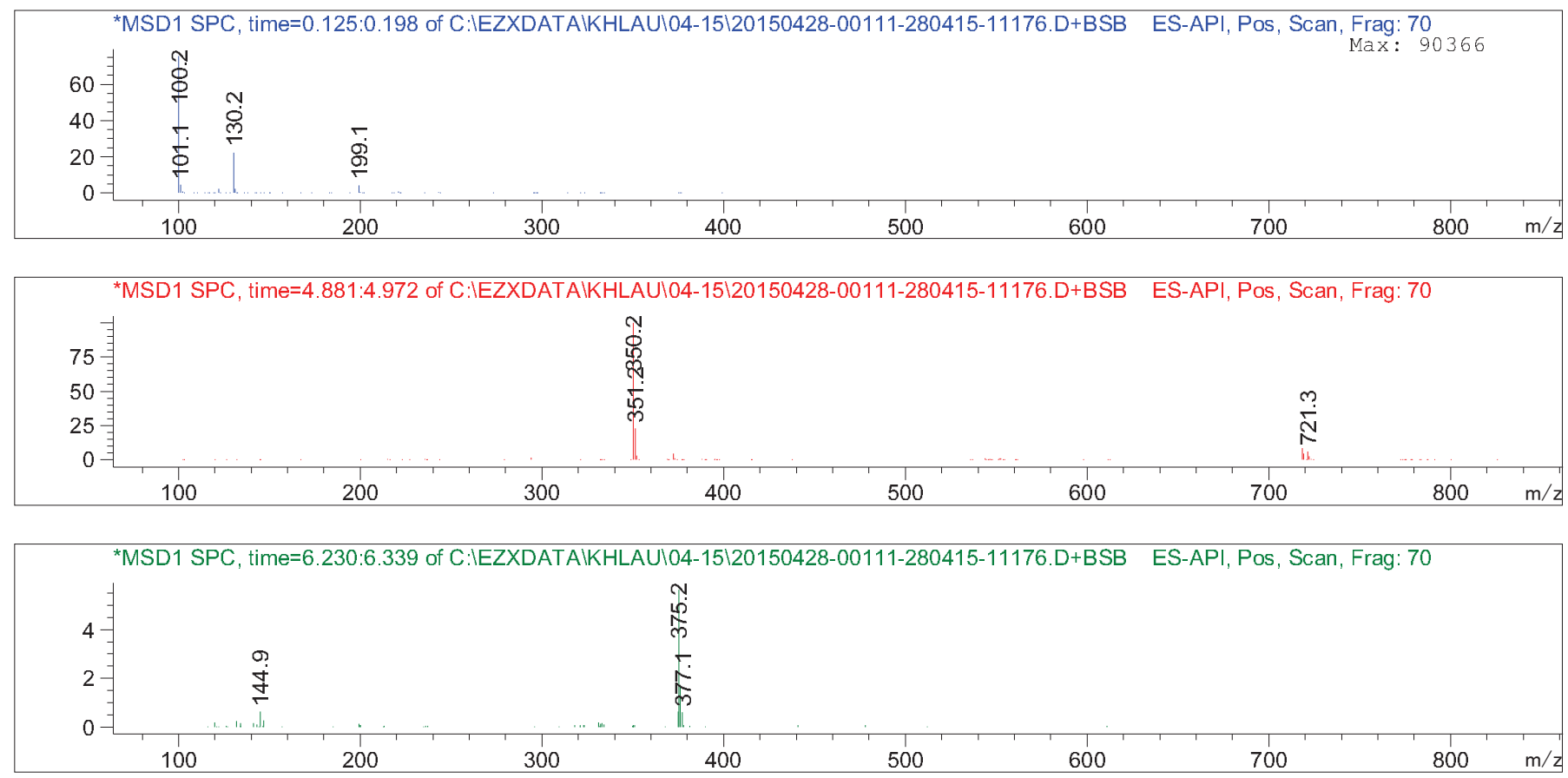


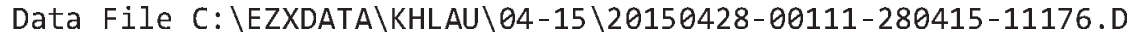

Sample Name: 20150428-0011
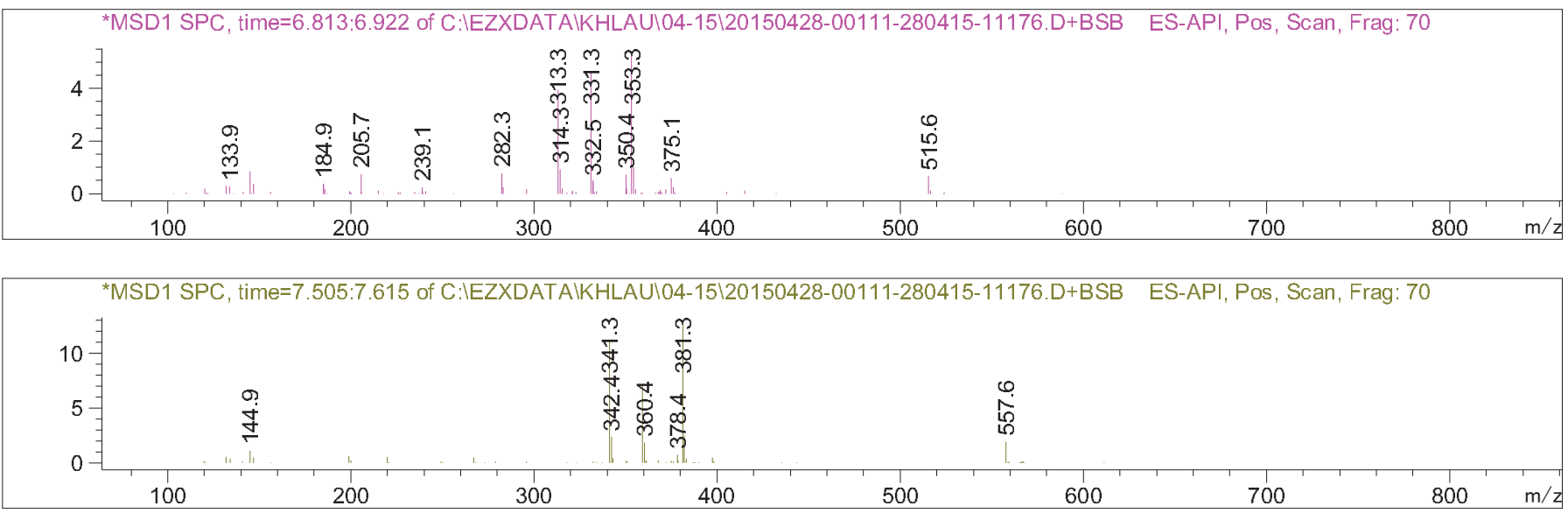


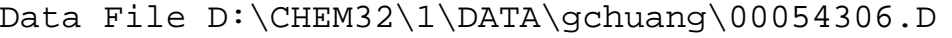

Sample Name: gc-0067-002
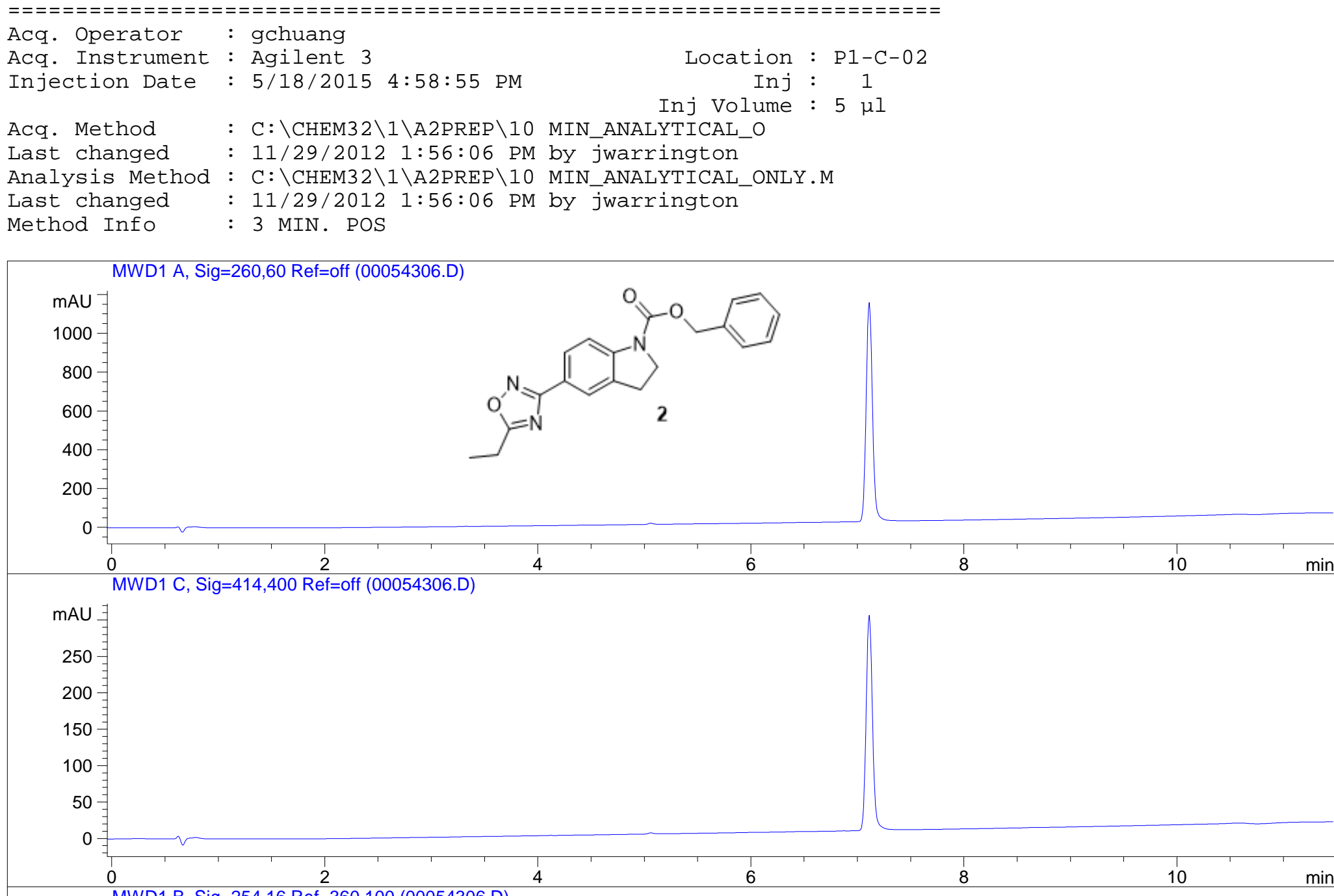
MWD1 B, Sig=254,16 Ref=360,100 (00054306.D)

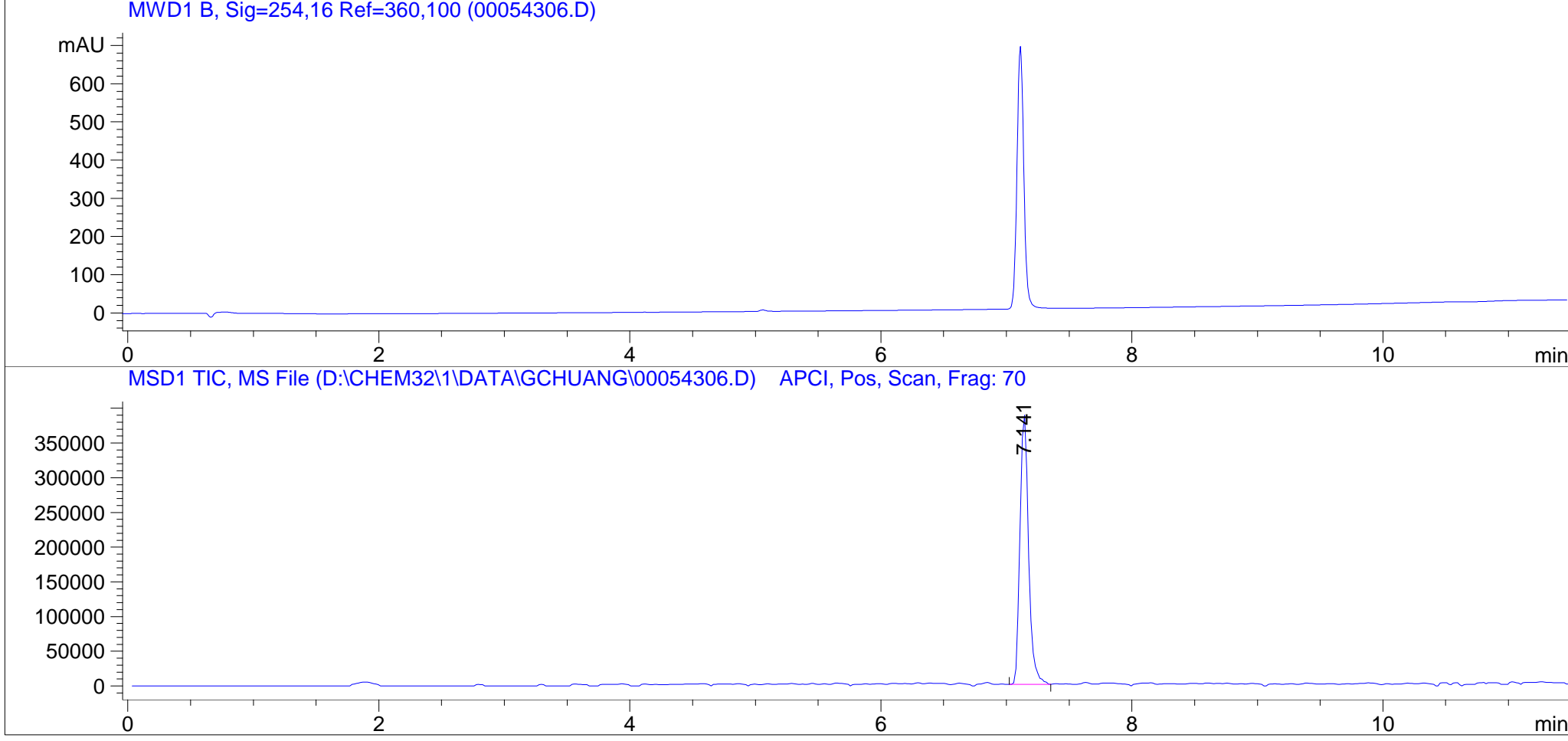




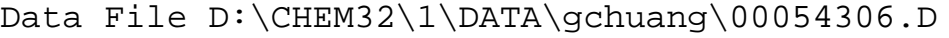

Sample Name: gc-0067-002

MS Signal: MSD1 TIC, MS File, APCI, Pos, Scan, Frag: 70 Spectra averaged over upper half of peaks. Noise Cutoff: 1000 counts.

Reportable Ion Abundance: > 10\%.

Retention

Time (MS)

7.141
MS Area

1857623
Mol. Weight

or Ion

$351.20 \mathrm{I}$

$350.20 \mathrm{I}$

$306.20 \mathrm{I}$

$223.20 \mathrm{I}$

$217.20 \mathrm{I}$

$216.20 \mathrm{I}$

$133.10 \mathrm{I}$

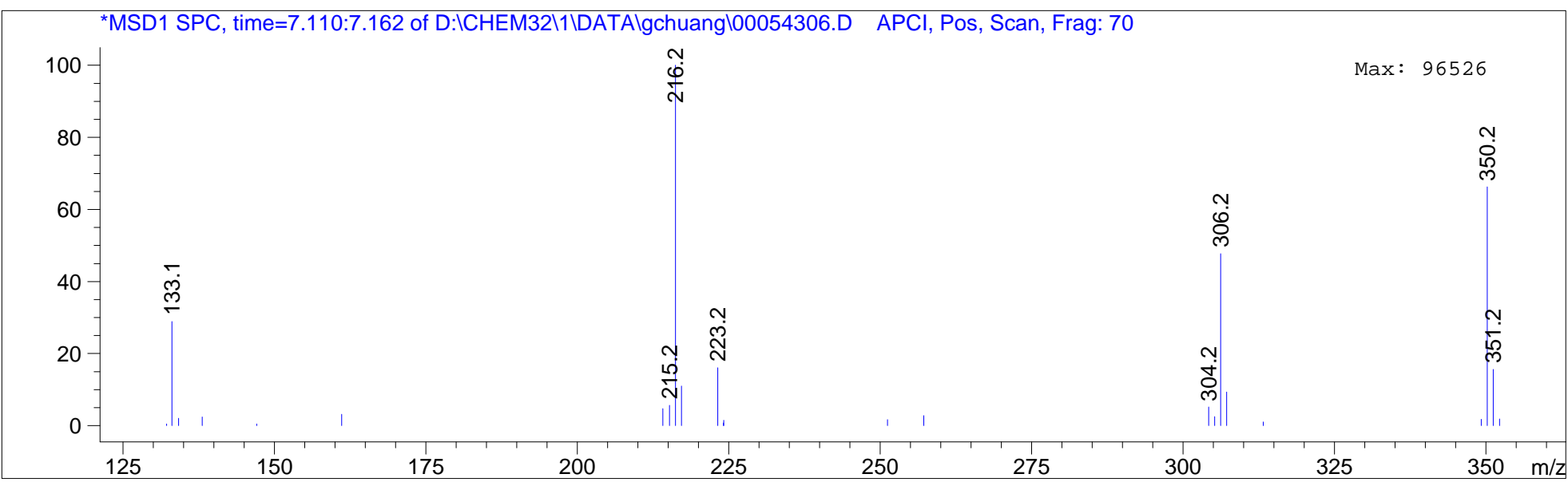




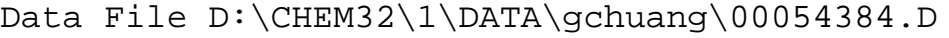

Sample Name: gc-0077-001
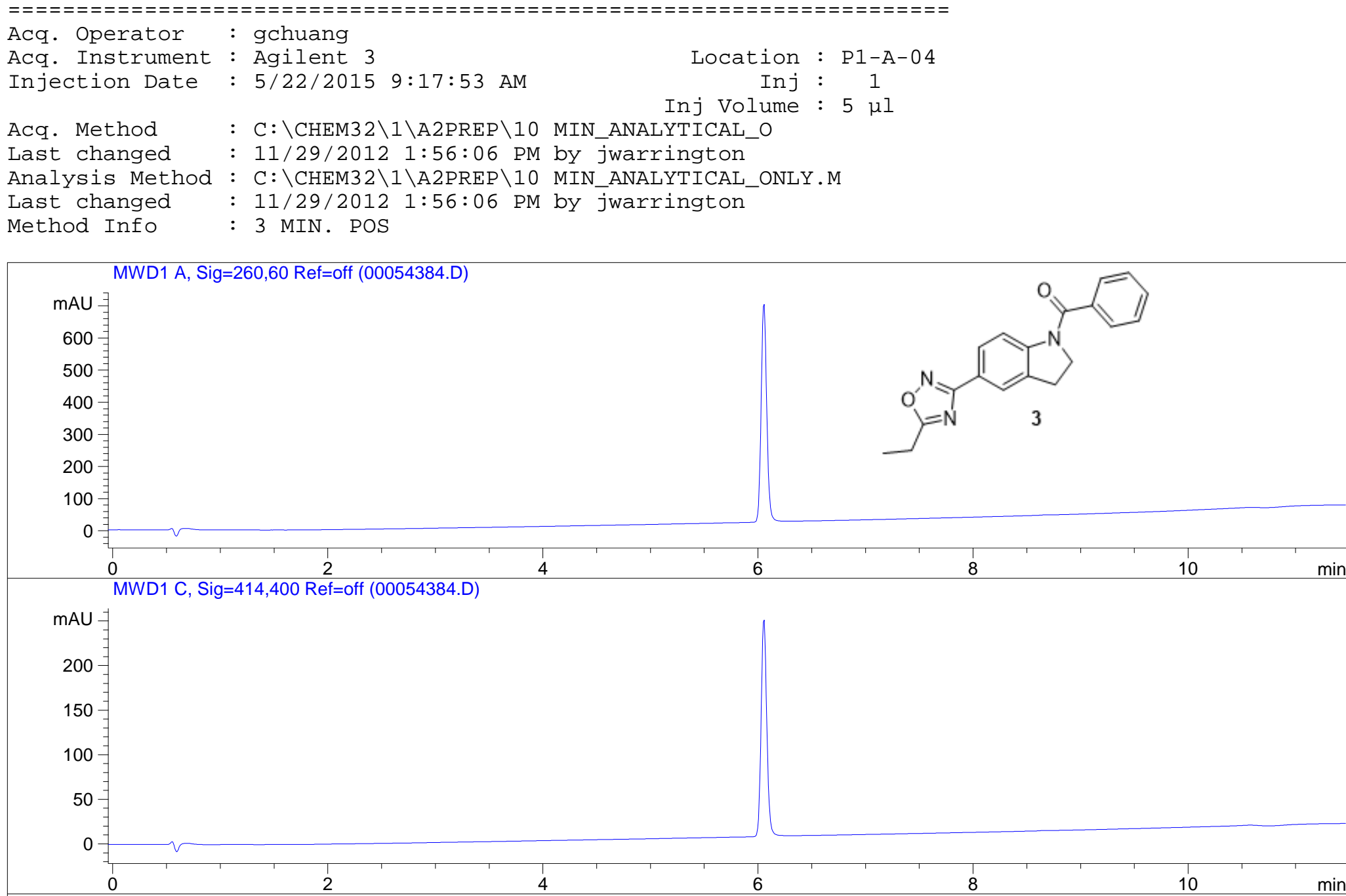
MWD1 B, Sig=254,16 Ref=360,100 (00054384.D)

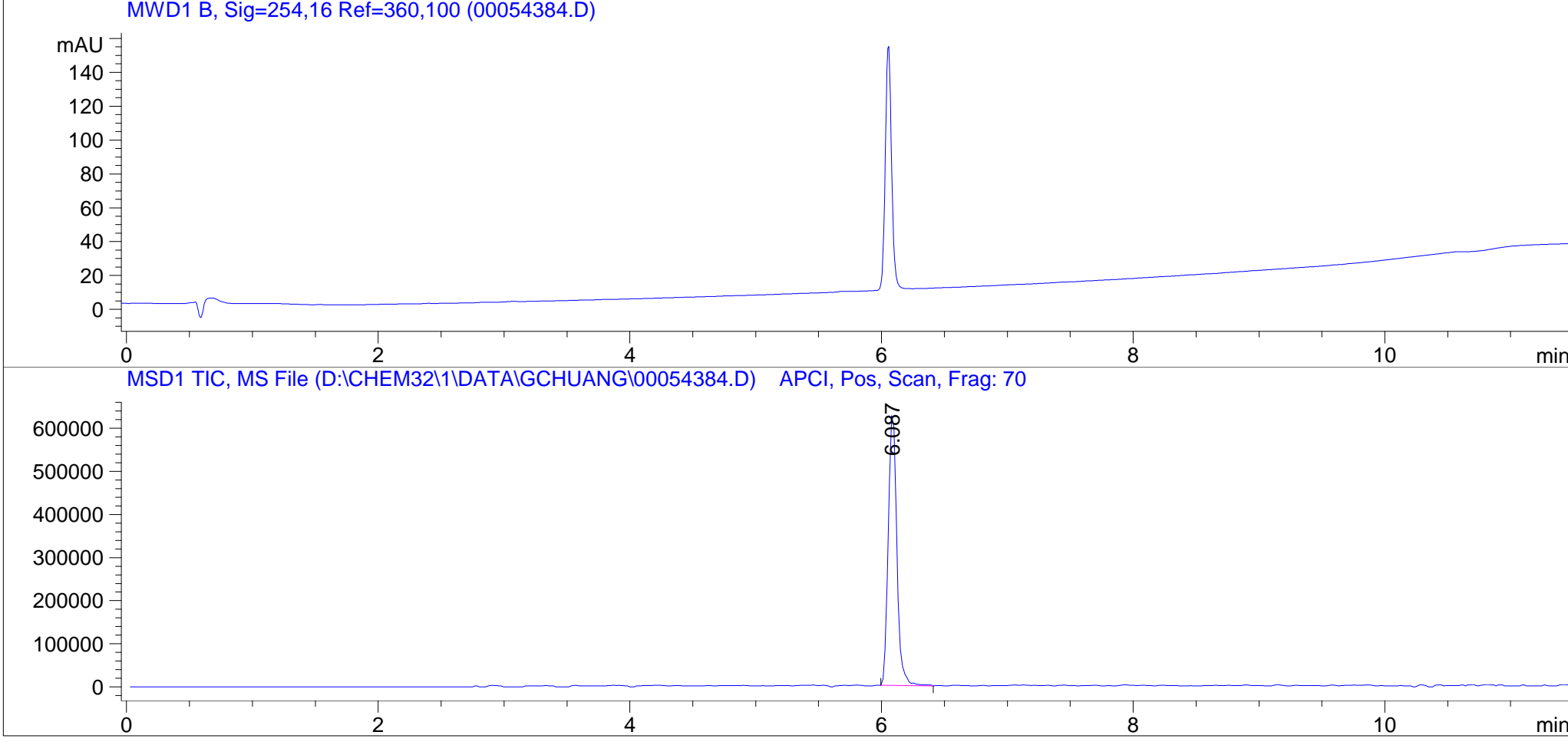




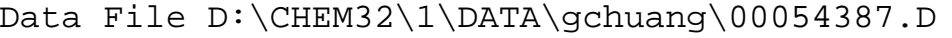

Sample Name: gc-0077-002
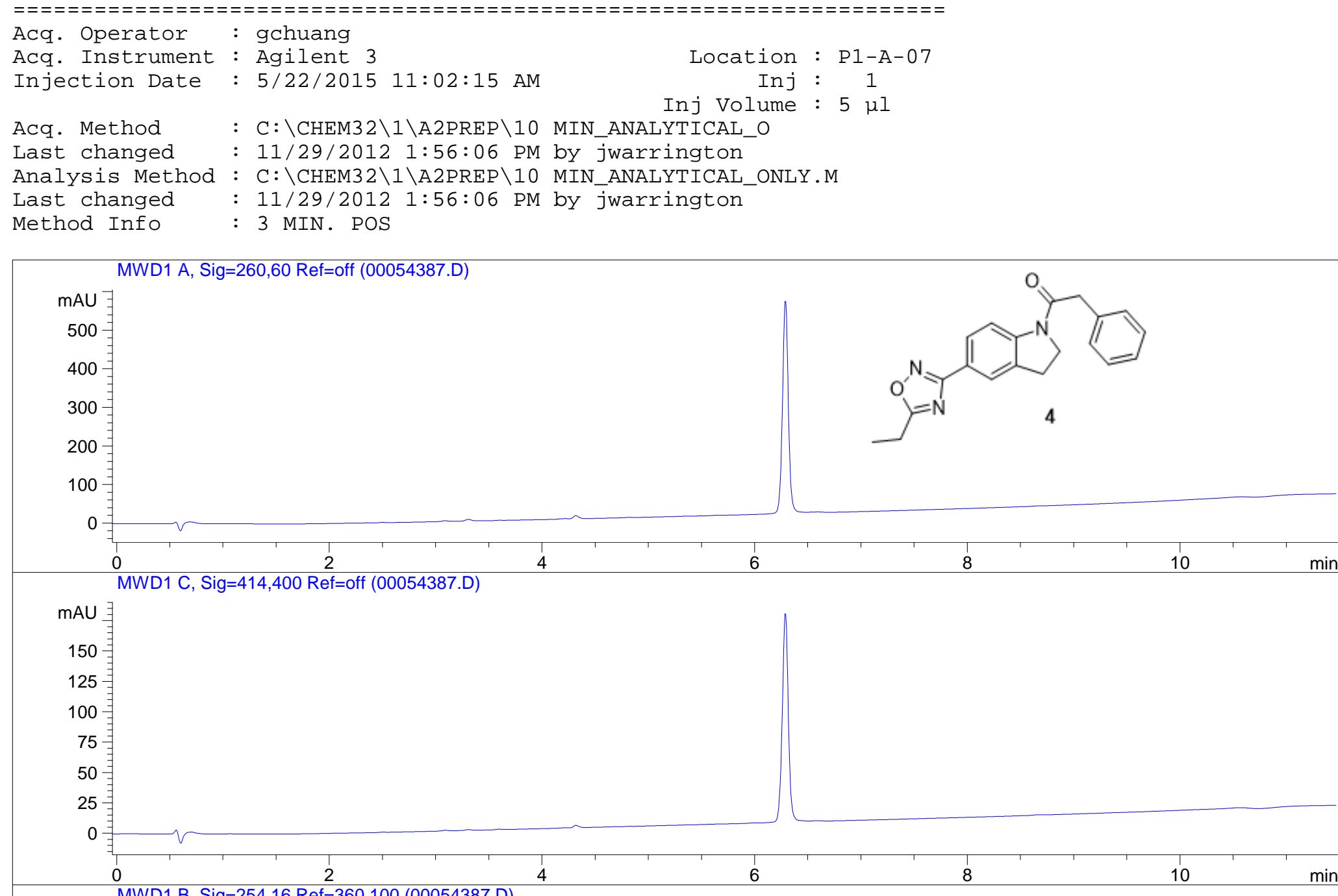
MWD1 B, Sig=254,16 Ref=360,100 (00054387.D)

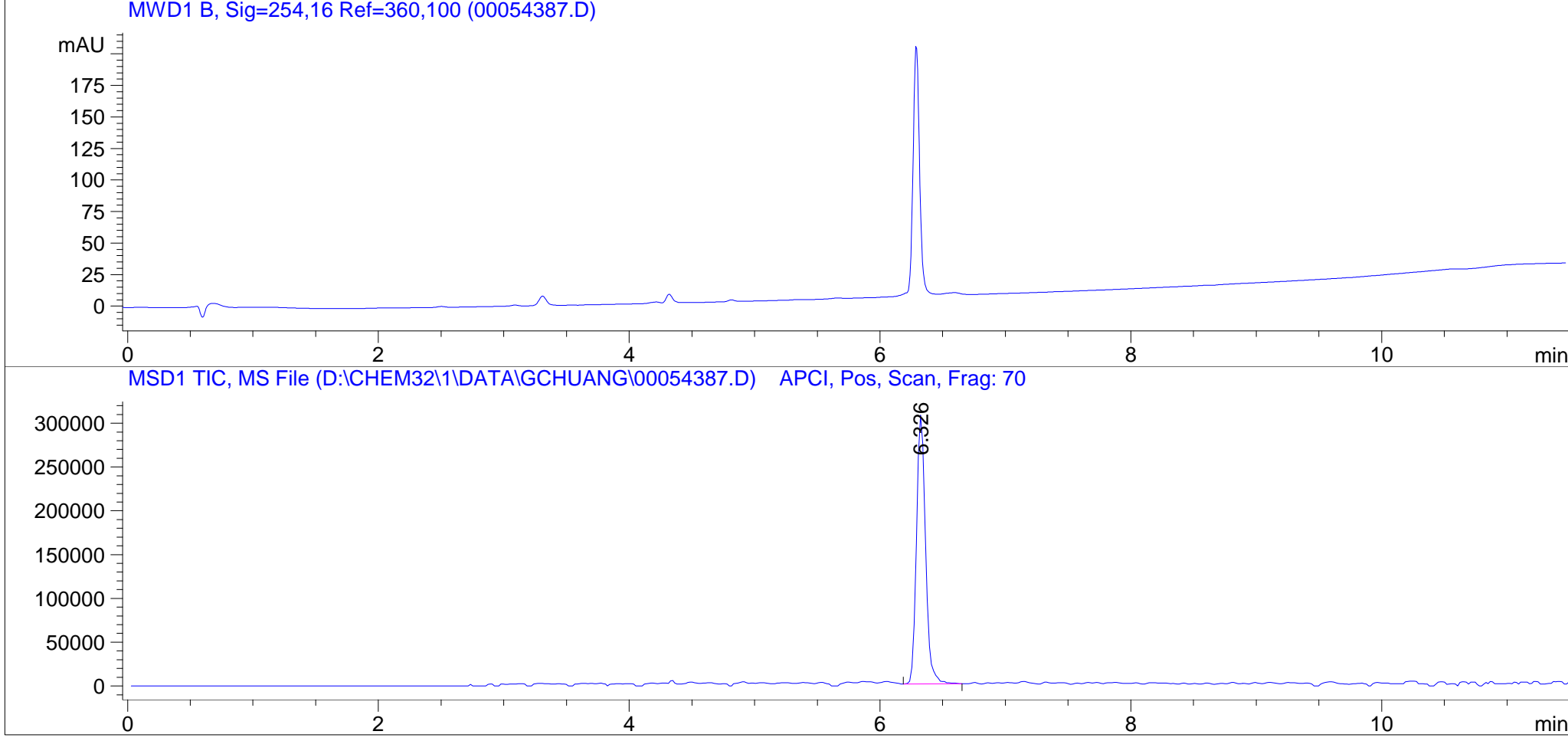




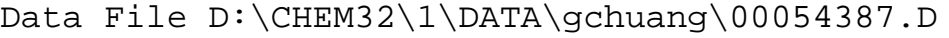

Sample Name: gc-0077-002

MS Signal: MSD1 TIC, MS File, APCI, Pos, Scan, Frag: 70 Spectra averaged over upper half of peaks. Noise Cutoff: 1000 counts.

Reportable Ion Abundance: > 10\%.

$\begin{array}{ccc}\begin{array}{l}\text { Retention } \\ \text { Time (MS) }\end{array} & \text { MS Area } & \text { Mol. Weight } \\ & & \text { or Ion } \\ 6.326 & 1541576 & 335.20 \mathrm{I} \\ & & 334.20 \mathrm{I}\end{array}$

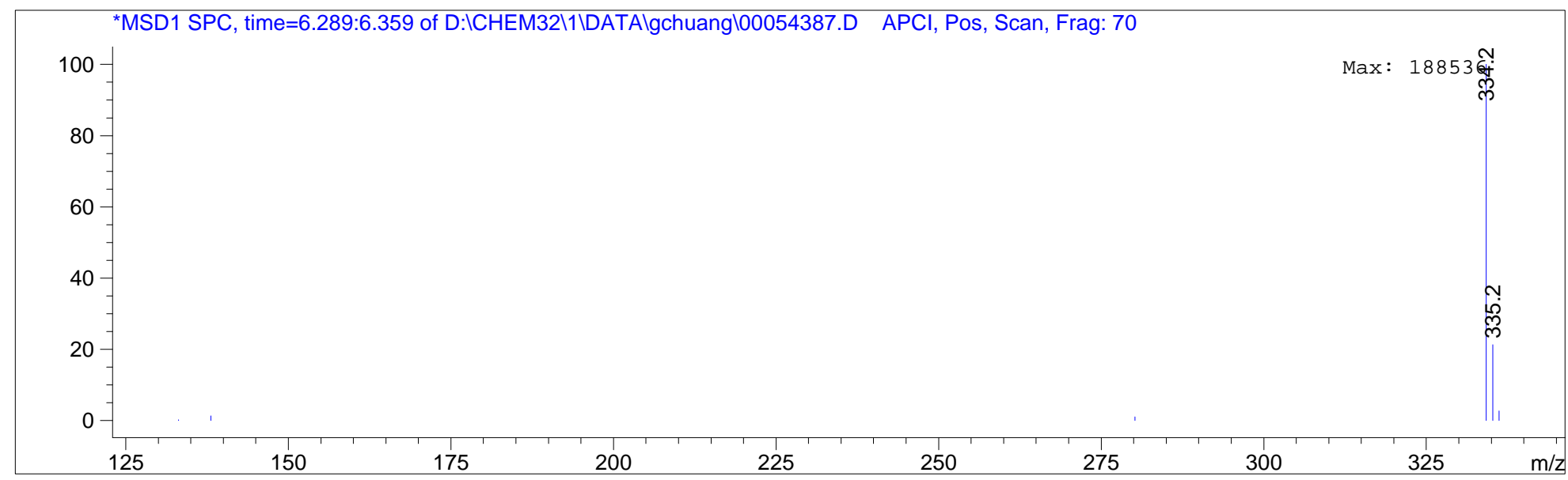




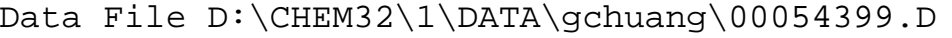

Sample Name: gc-0077-003
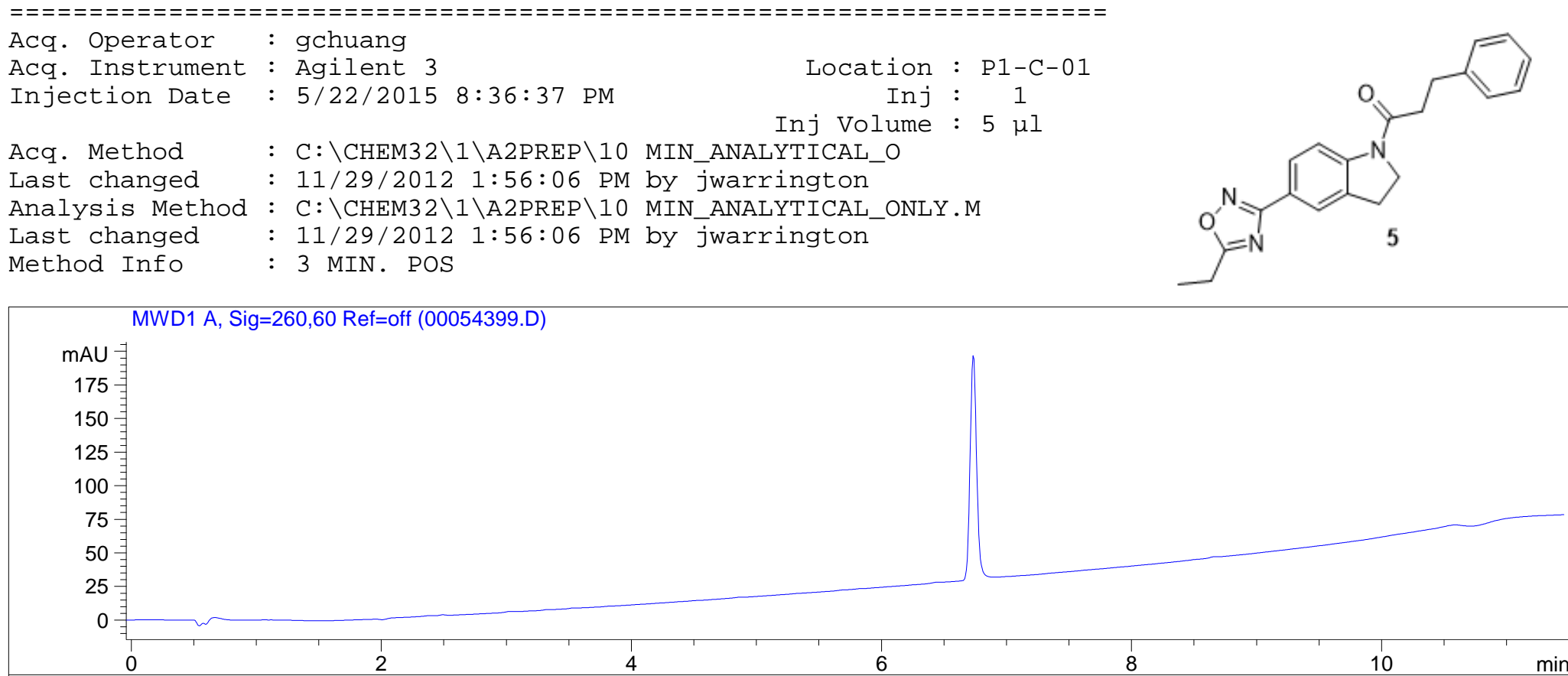

MWD1 C, Sig=414,400 Ref=off (00054399.D)

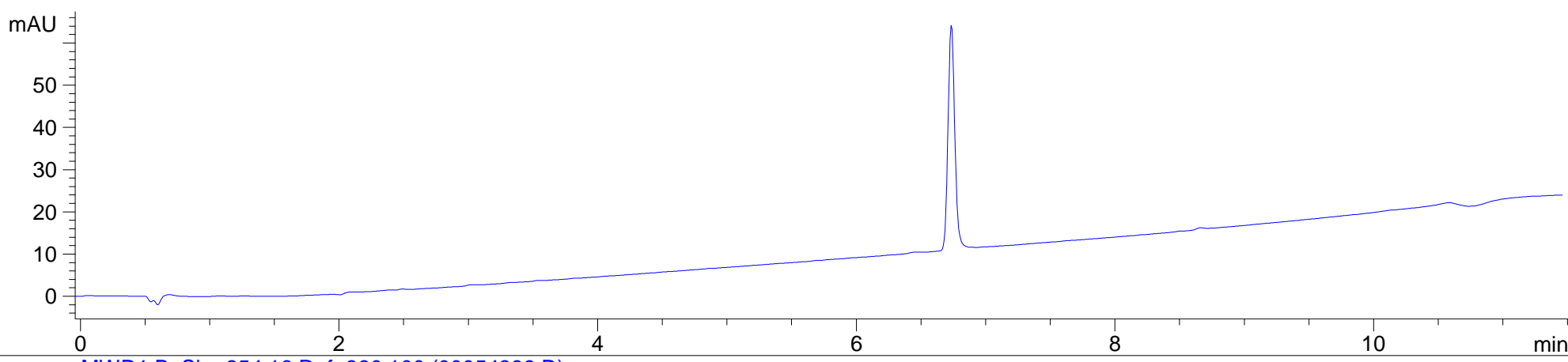

MWD1 B, Sig=254,16 Ref=360,100 (00054399.D)

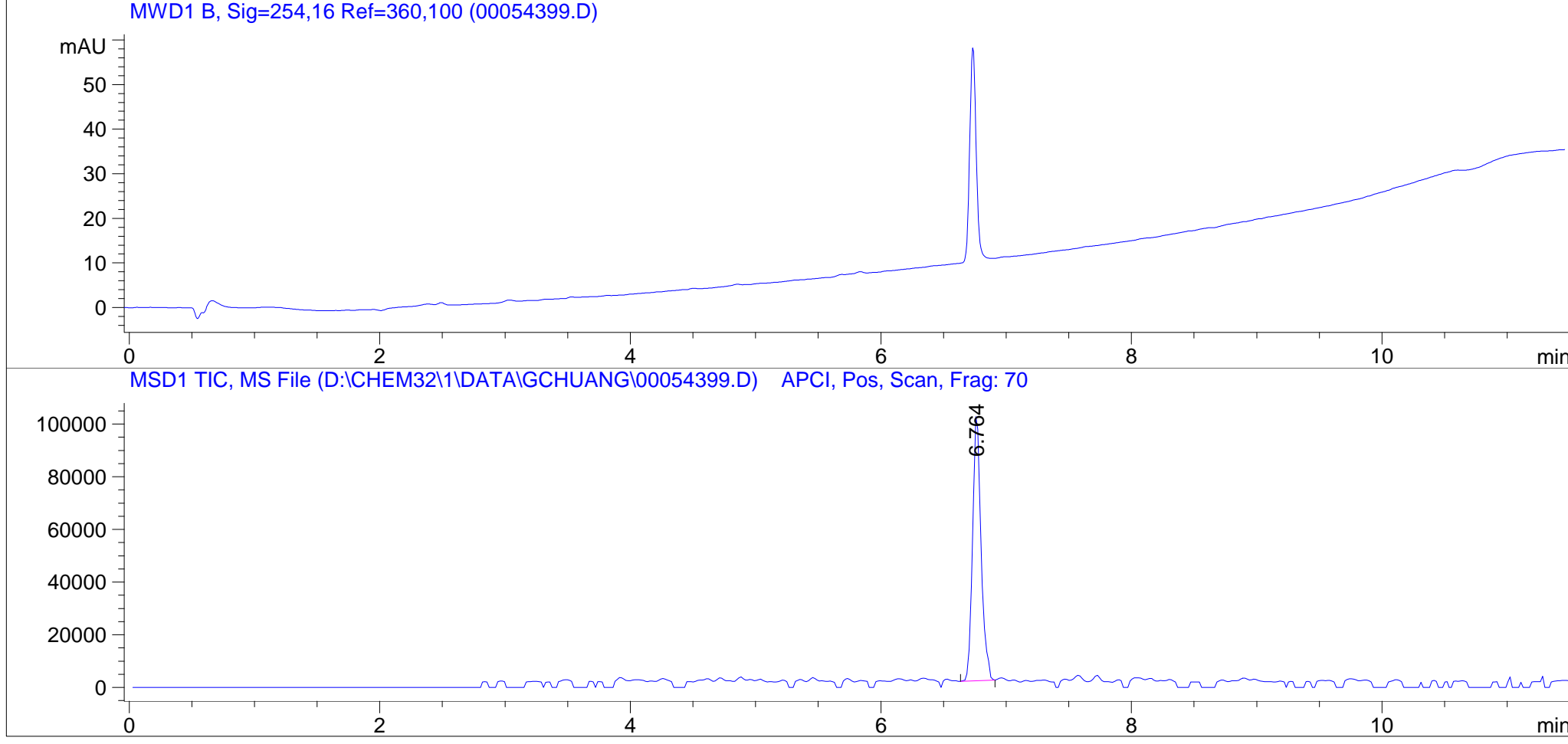




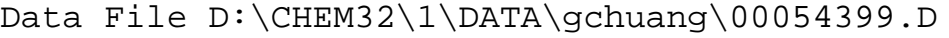

Sample Name: gc-0077-003

MS Signal: MSD1 TIC, MS File, APCI, Pos, Scan, Frag: 70 Spectra averaged over upper half of peaks. Noise Cutoff: 1000 counts.

Reportable Ion Abundance: > 10\%.

Retention

Time (MS)

6.764
MS Area

477074
Mol. Weight

or Ion

$349.20 \mathrm{I}$ $348.20 \mathrm{I}$

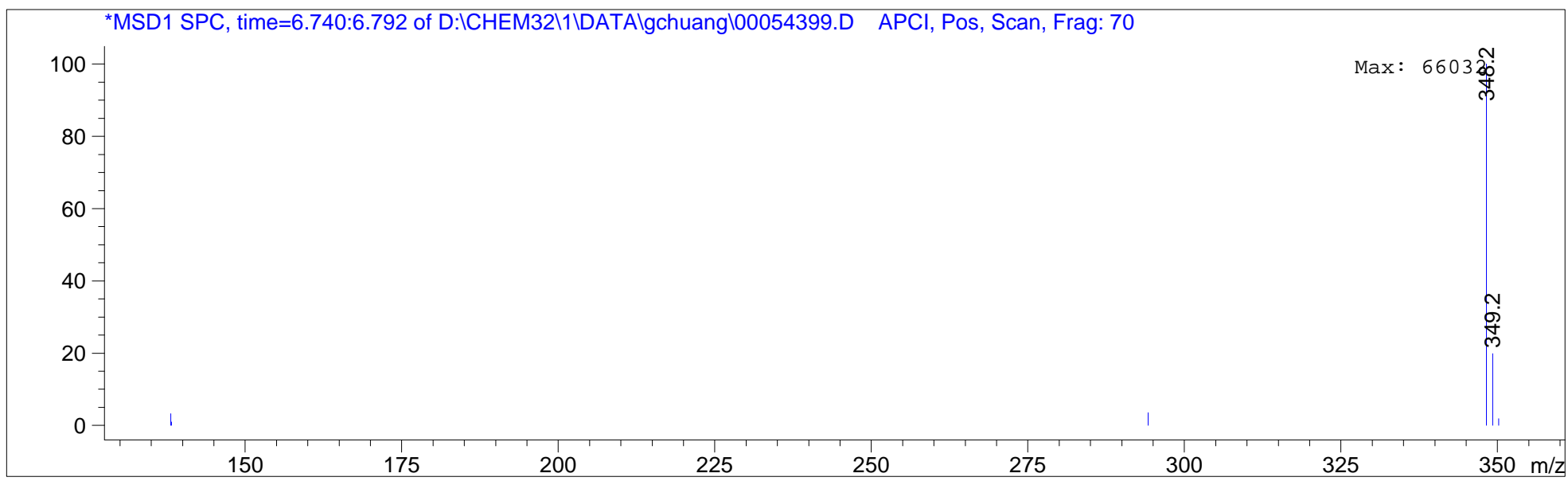




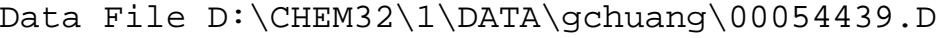

Sample Name: gc-0077-004
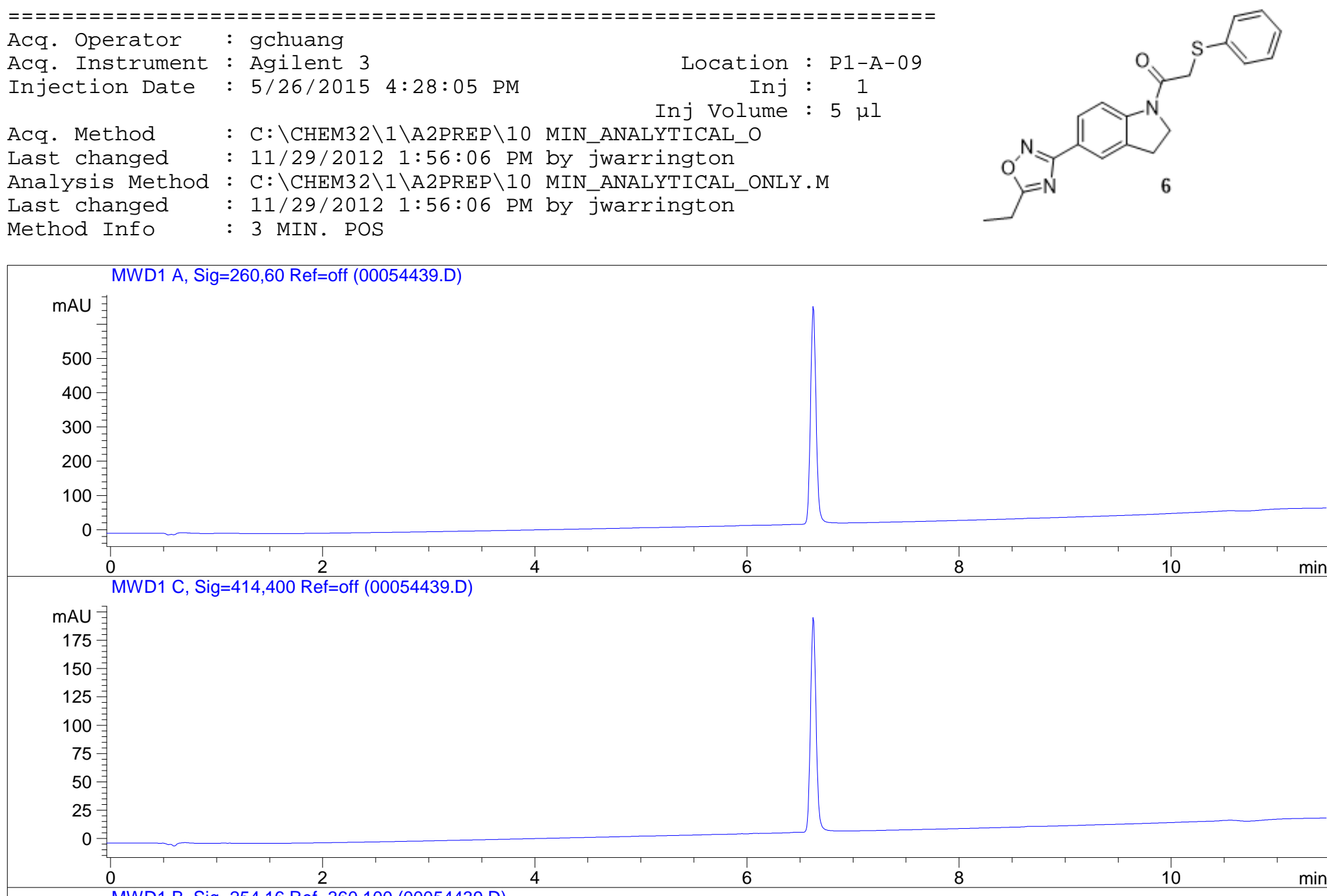
MWD1 B, Sig=254,16 Ref=360,100 (00054439.D)

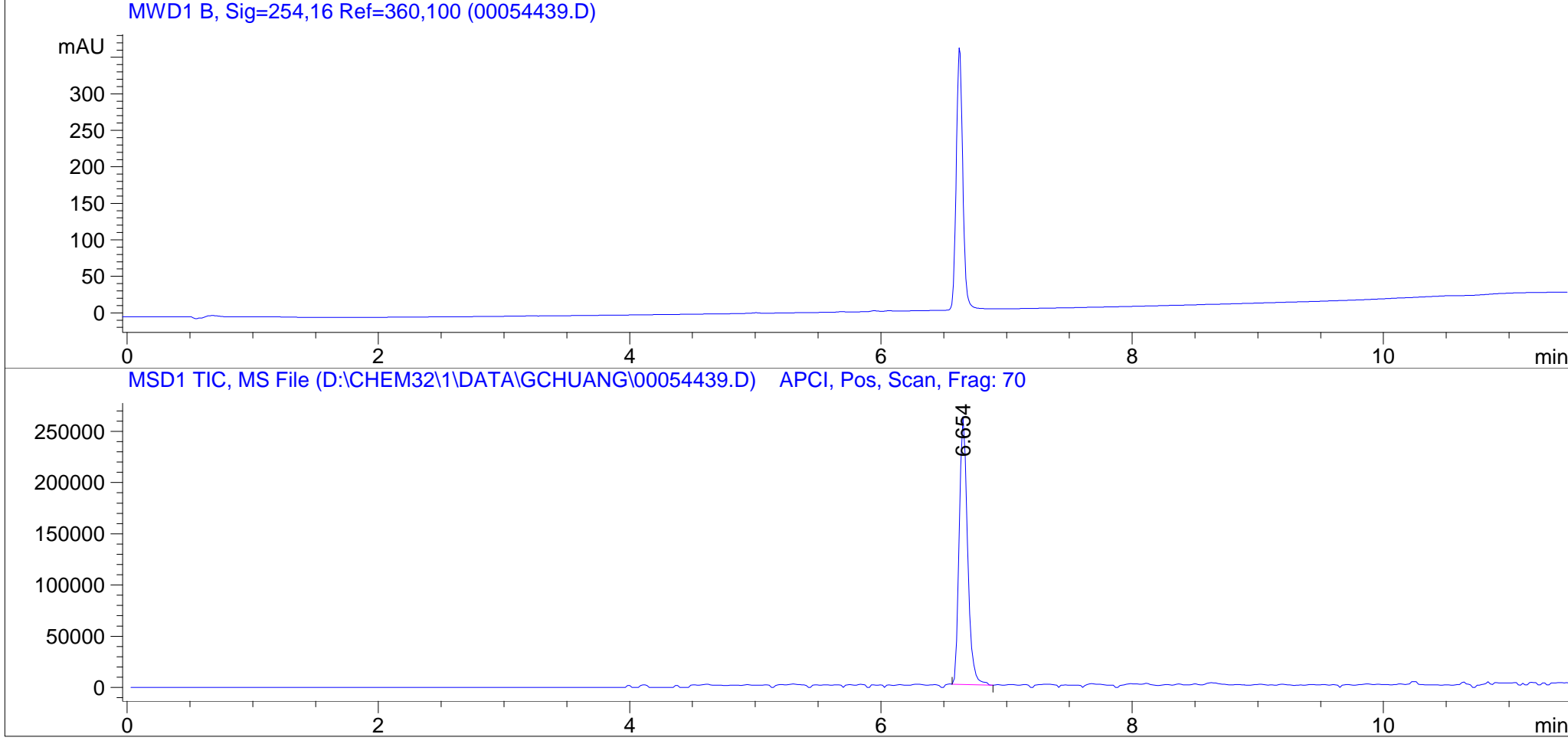




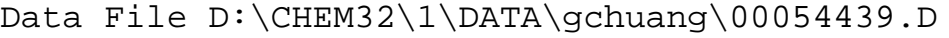

Sample Name: gc-0077-004

MS Signal: MSD1 TIC, MS File, APCI, Pos, Scan, Frag: 70 Spectra averaged over upper half of peaks. Noise Cutoff: 1000 counts.

Reportable Ion Abundance: > 10\%.

$\begin{array}{ccc}\begin{array}{l}\text { Retention } \\ \text { Time (MS) }\end{array} & \text { MS Area } & \text { Mol. Weight } \\ & & \text { or Ion } \\ 6.654 & 1197470 & 367.20 \mathrm{I} \\ & & 366.20 \mathrm{I}\end{array}$

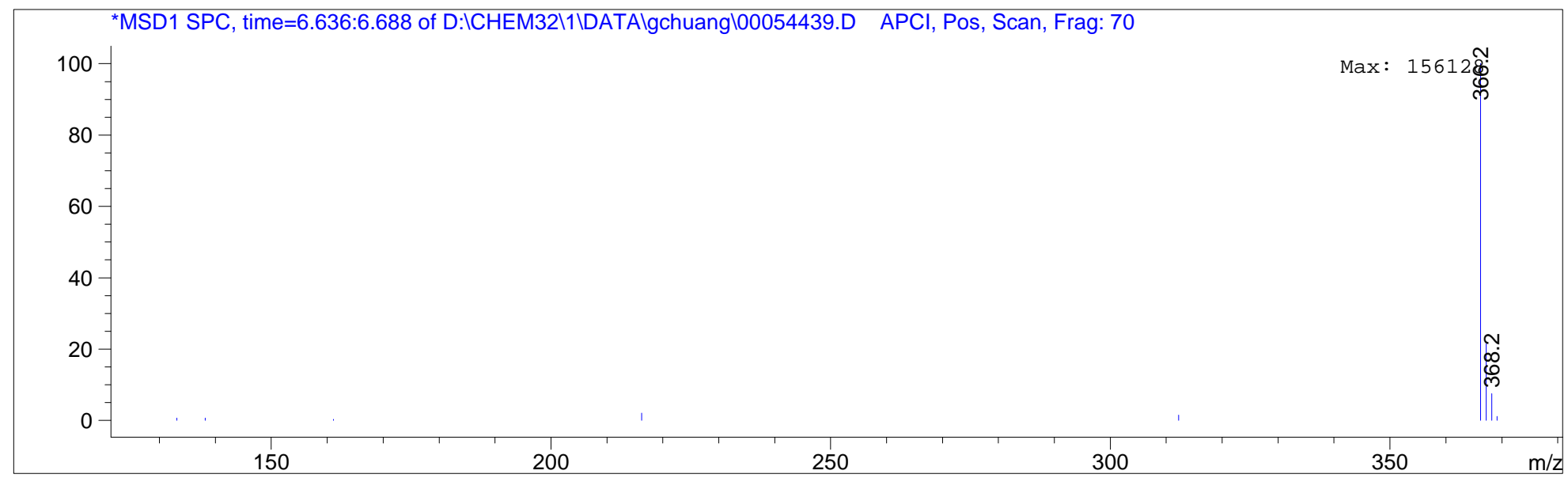




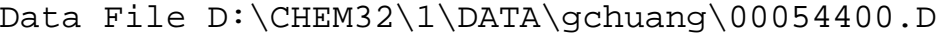

Sample Name: GC-0078-001
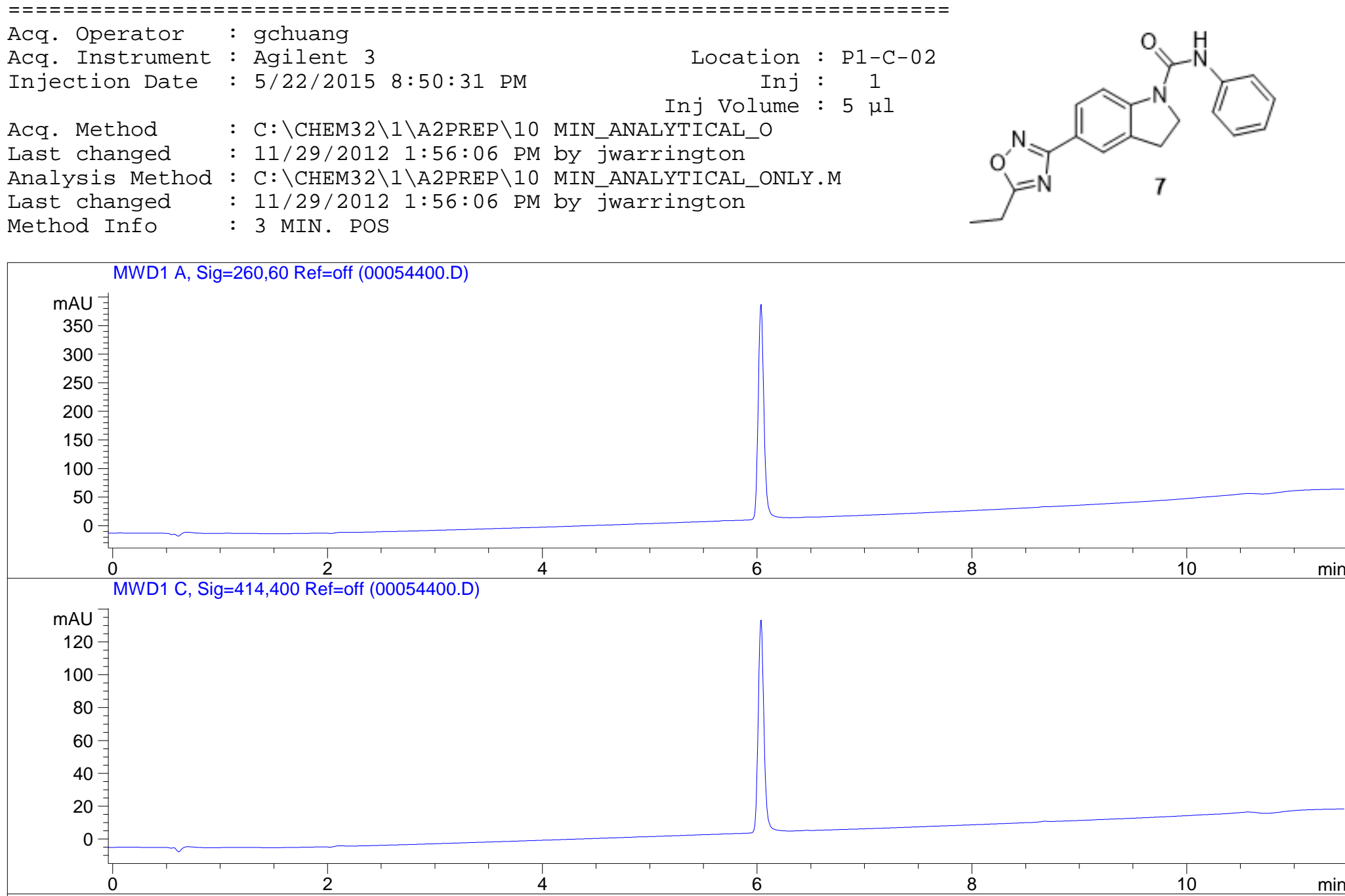

MWD1 B, Sig=254,16 Ref=360,100 (00054400.D)
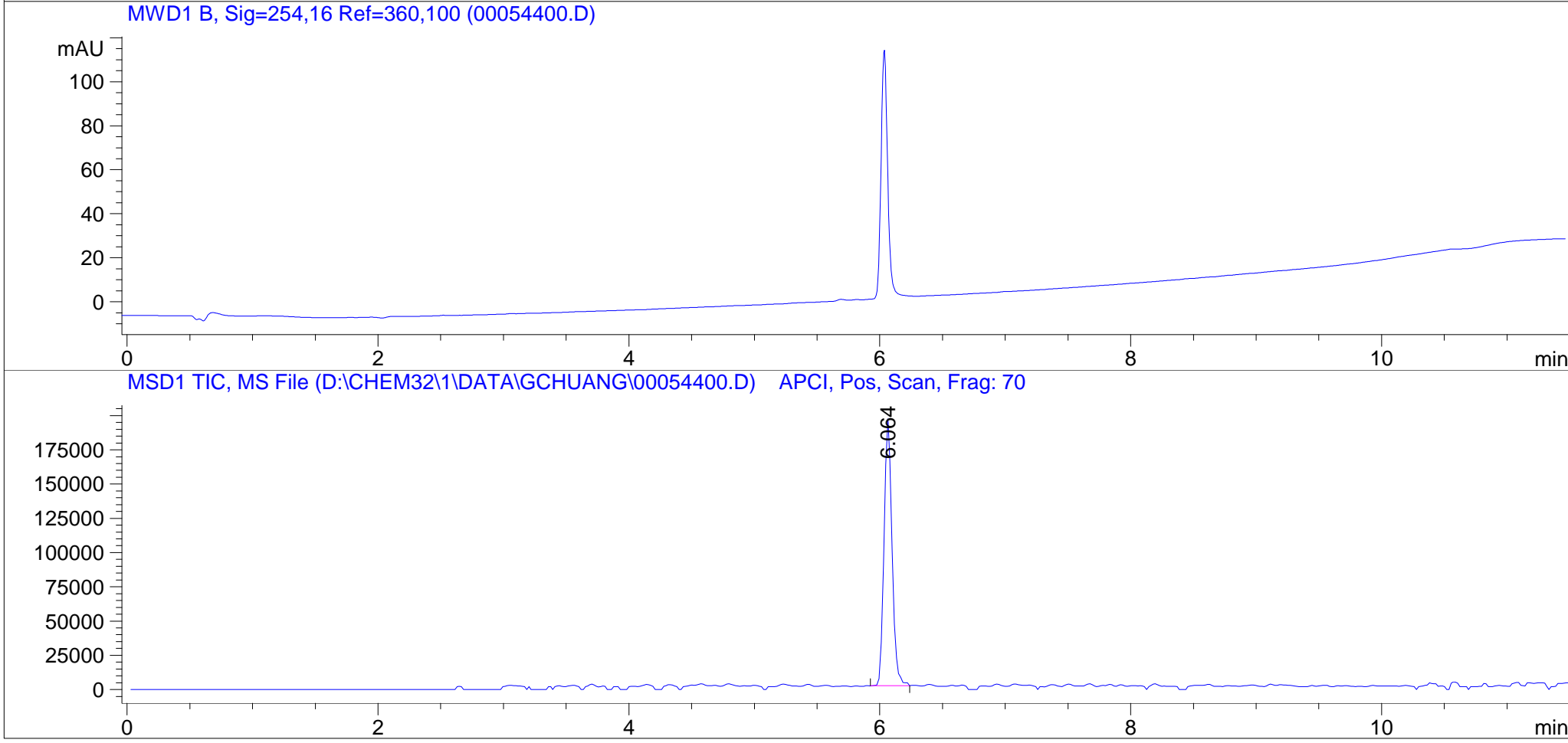


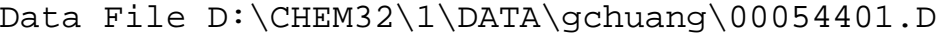

Sample Name: GC-0078-002
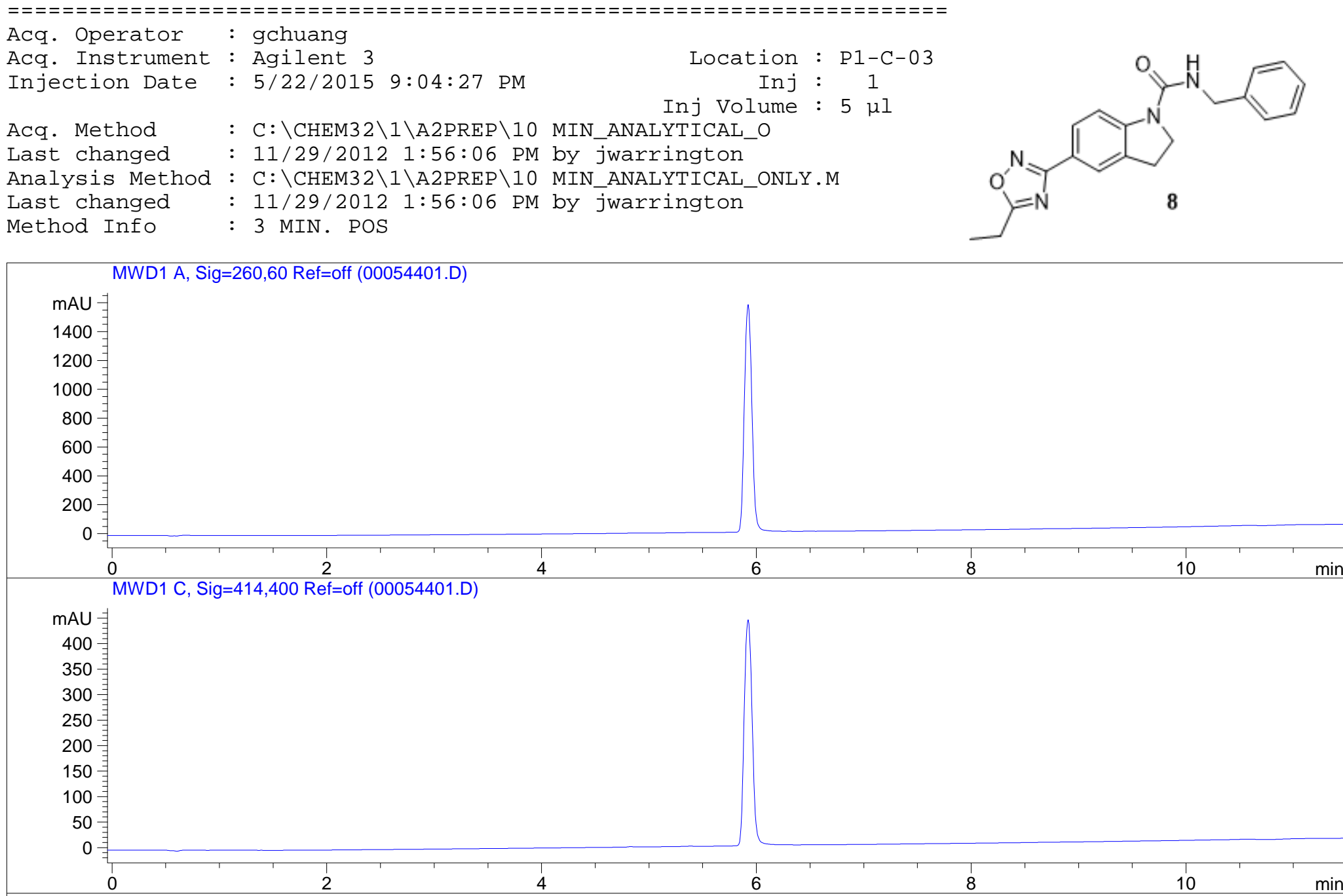

MWD1 B, Sig=254,16 Ref=360,100 (00054401.D)

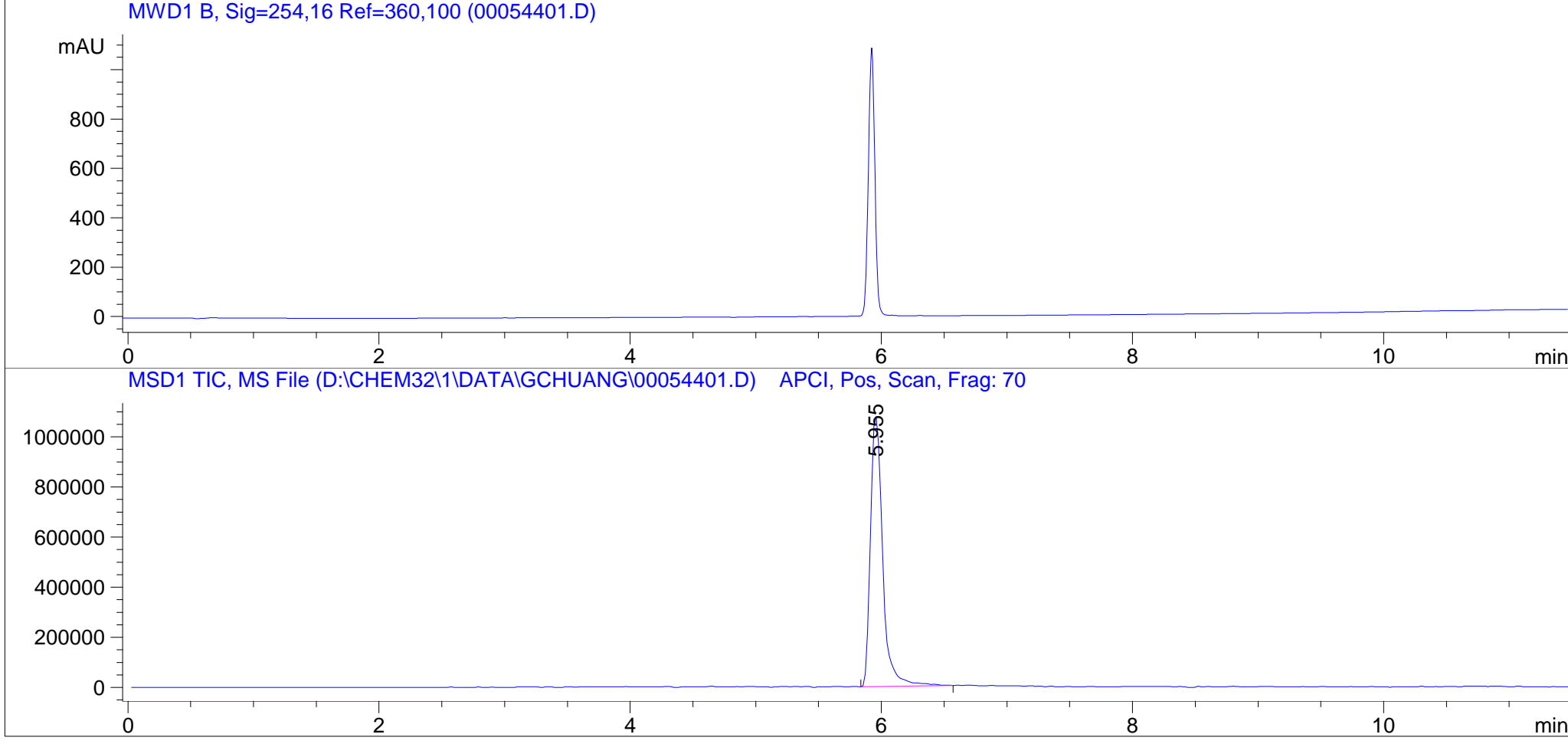




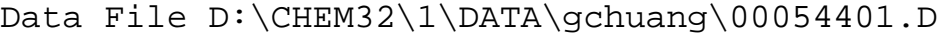

Sample Name: GC-0078-002

MS Signal: MSD1 TIC, MS File, APCI, Pos, Scan, Frag: 70 Spectra averaged over upper half of peaks. Noise Cutoff: 1000 counts.

Reportable Ion Abundance: > 10\%.

$\begin{array}{ccc}\begin{array}{l}\text { Retention } \\ \text { Time (MS) }\end{array} & \text { MS Area } & \begin{array}{l}\text { Mol. Weight } \\ \text { or Ion }\end{array} \\ & & \\ 5.955 & 7335686 & 350.20 \mathrm{I} \\ & & 349.20 \mathrm{I} \\ & 216.20 \mathrm{I}\end{array}$

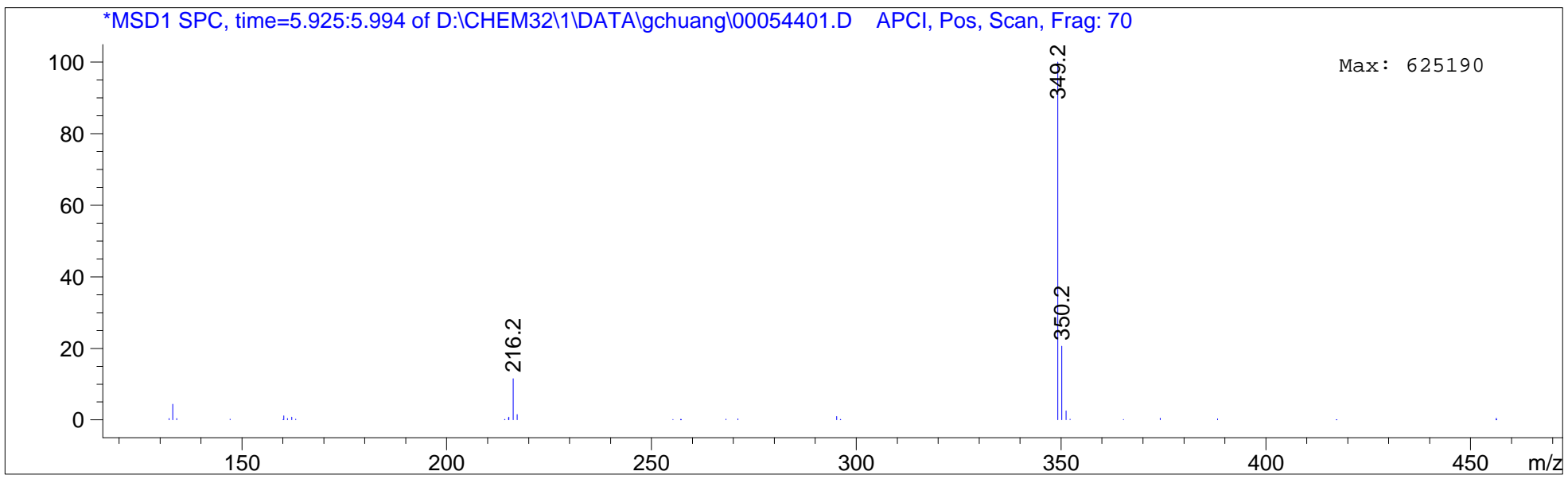




\section{LCMS Analysis Report}

\section{Acquired by \\ Sample Name Injection Volume \\ Data File \\ Method File \\ Report Format File \\ Date Acquired \\ Comment}

\author{
: System Administrator \\ : LCMS41-PH-CYT-A-637-0-4(57214-056A1)1T \\ $: 5$ \\ : LCMS41-PH-CYT-A-637-0-4(57214-056A1)1T.Icd \\ : ACN-Water-0.1\%FA-30\%B-1.5-5.0MIN(90-900).Icm \\ : LCMS2020-PDA+ELS+TIC+MS.Isr \\ : 2015-10-13 15:22:12 \\ : Mobile phase A:Water/0.1\% FA \\ Mobile phase B:Acetonitrile/0.1\% FA
}

$\begin{array}{ll}\text { Instrument Name:Shimadzu LCMS-2020 } \\ \begin{array}{l}<\text { Pump }>> \\ \text { Mode }\end{array} & : \text { Binary gradient } \\ \text { Pump A } & : \text { LC-30AD } \\ \text { Pump B } & : \text { LC-30AD } \\ \text { Total Flow } & : 1.5000 \mathrm{~mL} / \mathrm{min} \\ \text { B Conc. } & : 30.0 \% \\ & \\ \text { <<Oven>> } & : 40 \mathrm{C} \\ \text { Oven Temperature } & \\ & \\ \text { <<PDA >> } & : \text { SPD-M20A } \\ \text { PDA Model } & : \text { D2 } \\ \text { Lamp } & : 190 \mathrm{~nm} \\ \text { Start Wavelength } & : 400 \mathrm{~nm} \\ \text { End Wavelength } & \end{array}$

$\begin{array}{ll}\text { <<Interface>> } & \\ \text { Interface } & : E S I \\ \text { DL Temperature } & : 250 \mathrm{C} \\ \text { Nebulizing Gas Flow } & : 1.50 \mathrm{~L} / \mathrm{min} \\ \text { Heat Block } & : 280 \mathrm{C} \\ \text { Drying Gas } & : \text { On } \\ & 15.00 \mathrm{~L} / \mathrm{min} \\ \text { <<MS Parameter> } & \\ \text {--Segment 1 Event } 1-- & : 0.00 \mathrm{~min} \\ \text { Start Time } & : 5.00 \mathrm{~min} \\ \text { End Time } & : S c a n \\ \text { Acquisition Mode } & : P 0 \text { itive } \\ \text { Polarity } & : 1.00 \mathrm{sec} \\ \text { Event Time } & :+1.15 \mathrm{kV} \\ \text { Detector Voltage } & : 0 \\ \text { Threshold } & : 90.00 \\ \text { Start m/z } & : 900.00 \\ \text { End m/z } & : 834 \mathrm{u} / \mathrm{sec} \\ \text { Scan Speed } & : U s e \text { the Data in the Tuning File } \\ \text { Interface Volt. } & : \text { Use the Data in the Tuning File } \\ \text { DL Volt. } & : \text { Use the Data in the Tuning File } \\ \text { Qarray DC Voltage } & : \text { Use the Data in the Tuning File } \\ \text { Qarray DC Voltage } & \end{array}$

System Configuration

$<<$ Column $>>$

Column Name

Length

Internal Diameter

Description

$<<$ LC Time Program $>>$
Time
0.01
4.00
4.70
4.80
5.00

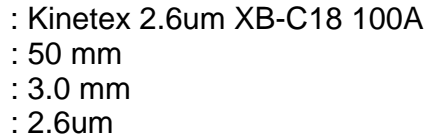

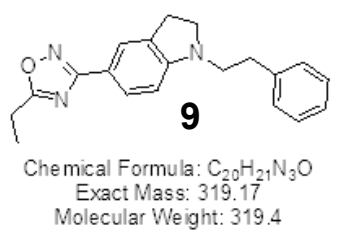

ES

$1.50 \mathrm{~L} / \mathrm{min}$

$: 280 \mathrm{C}$

:On

$: 0.00 \mathrm{~min}$

:5.00 min

$: 1.00 \mathrm{sec}$

$15 \mathrm{kV}$

:90.00

:Use the Data in the Tuning File

$\begin{array}{lll}\text { Module } & \text { Command } & \text { Value } \\ \text { Pumps } & \text { Pump B Conc. } & 30 \\ \text { Pumps } & \text { Pump B Conc. } & 100 \\ \text { Pumps } & \text { Pump B Conc. } & 100 \\ \text { Pumps } & \text { Pump B Conc. } & 30 \\ \text { Controller } & \text { Stop } & \end{array}$


mAU

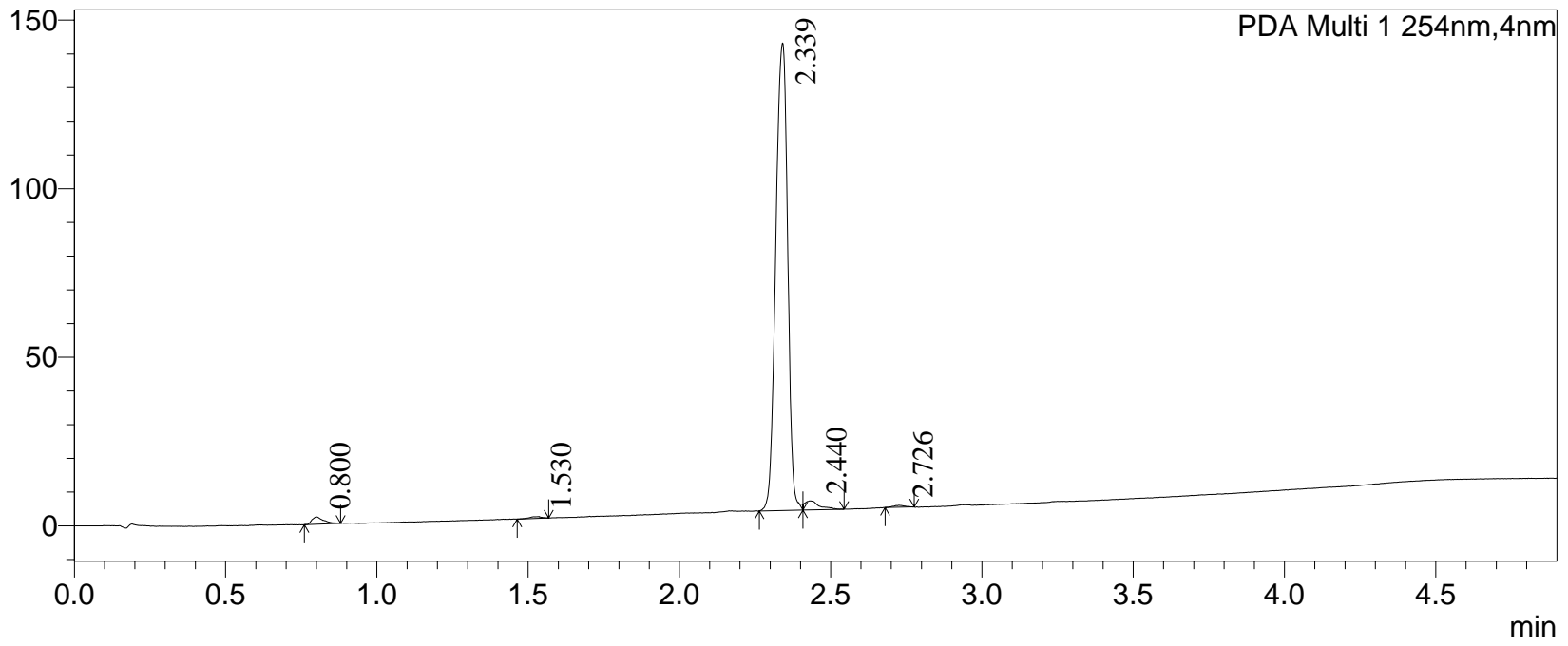

Peak Table

PDA Ch1 254nm

\begin{tabular}{|r|r|r|r|r|r|}
\hline Peak\# & Ret. Time & \multicolumn{1}{|c|}{ Height } & Height\% & \multicolumn{1}{|c|}{ Area } & \multicolumn{1}{c|}{ Area\% } \\
\hline 1 & 0.800 & 2082 & 1.450 & 5768 & 1.458 \\
\hline 2 & 1.530 & 490 & 0.341 & 1446 & 0.365 \\
\hline 3 & 2.339 & 137900 & 96.024 & 378090 & 95.533 \\
\hline 4 & 2.440 & 2594 & 1.806 & 8954 & 2.262 \\
\hline 5 & 2.726 & 545 & 0.379 & 1511 & 0.382 \\
\hline Total & & 143610 & 100.000 & 395769 & 100.000 \\
\hline
\end{tabular}

mAU

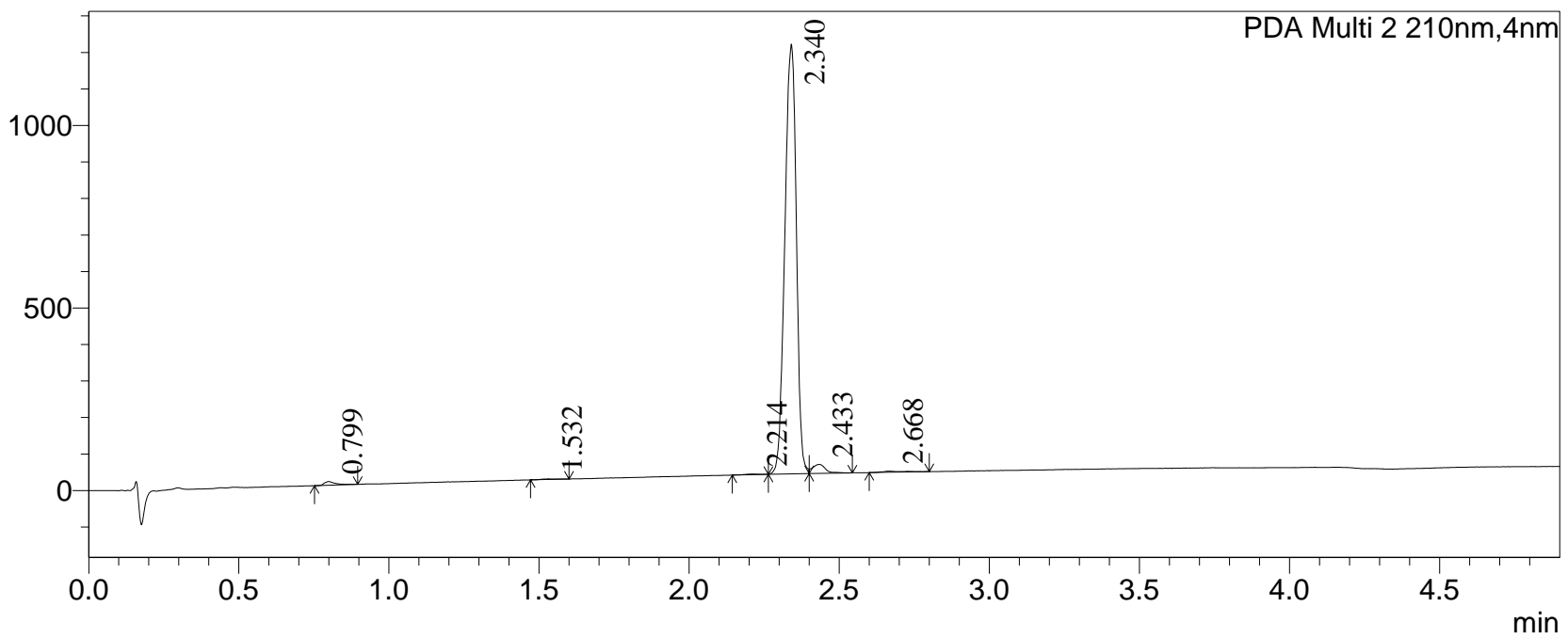

PDA Ch2 210nm

Peak Table

\begin{tabular}{|r|r|r|r|r|r|}
\hline Peak\# & \multicolumn{1}{|c|}{ Ret. Time } & \multicolumn{1}{c|}{ Height } & Height\% & \multicolumn{1}{c|}{ Area } & \multicolumn{1}{c|}{ Area\% } \\
\hline 1 & 0.799 & 9793 & 0.810 & 26336 & 0.809 \\
\hline 2 & 1.532 & 1702 & 0.141 & 5413 & 0.166 \\
\hline 3 & 2.214 & 1690 & 0.140 & 5435 & 0.167 \\
\hline 4 & 2.340 & 1169295 & 96.664 & 3130828 & 96.137 \\
\hline 5 & 2.433 & 24245 & 2.004 & 74253 & 2.280 \\
\hline 6 & 2.668 & 2925 & 0.242 & 14375 & 0.441 \\
\hline Total & & 1209649 & 100.000 & 3256641 & 100.000 \\
\hline
\end{tabular}


$\mathrm{mV}$

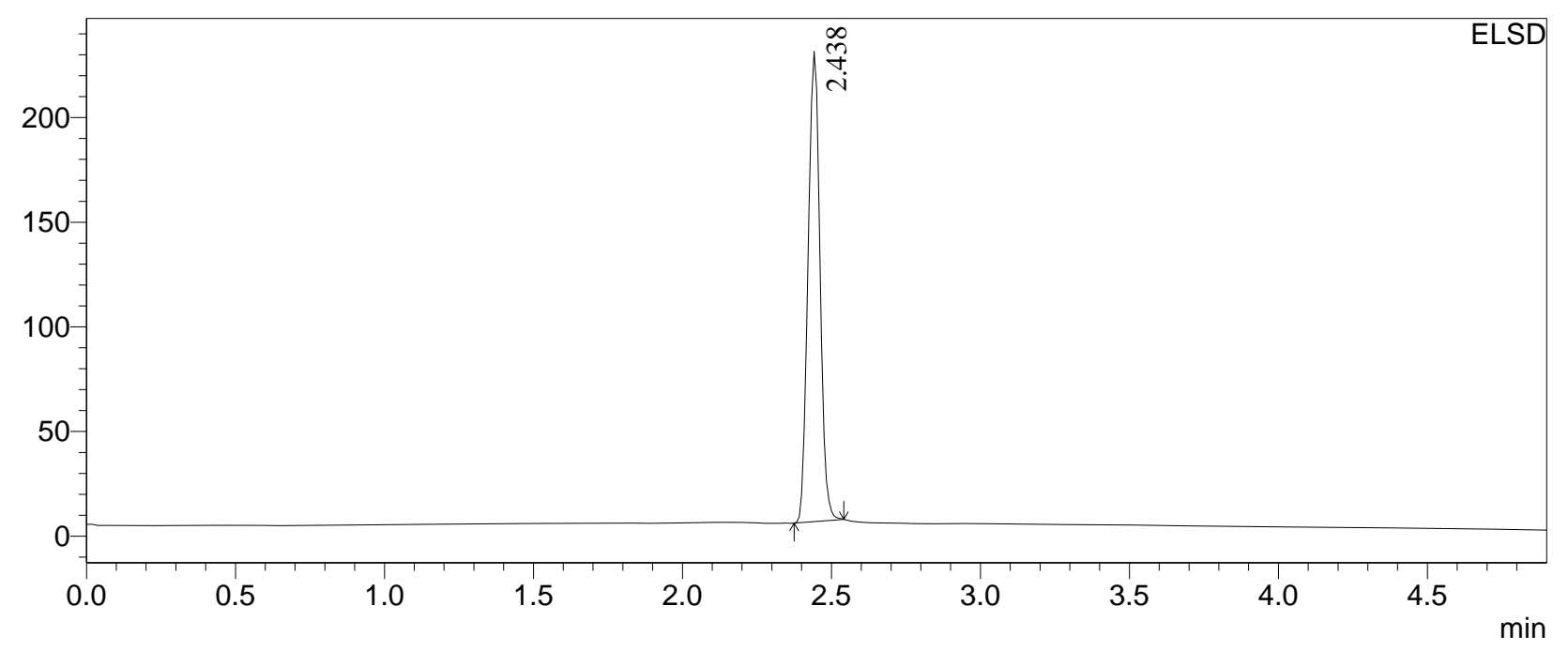

Peak Table

ELSD

\begin{tabular}{|r|r|r|r|r|r|}
\hline Peak\# & Ret. Time & Height & \multicolumn{1}{c|}{ Height\% } & \multicolumn{1}{c|}{ Area } & \multicolumn{1}{c|}{ Area\% } \\
\hline 1 & 2.438 & 224798 & 100.000 & 624549 & 100.000 \\
\hline Total & & 224798 & 100.000 & 624549 & 100.000 \\
\hline
\end{tabular}

Line\#:1 R.Time:2.342(Scan\#:145)

Mass Spectrum

MassPeaks: 468

Spectrum Mode:Single 2.342(145) BasePeak:320.1(5237826)

BG Mode:Averaged 2.258-2.492(140-154) Segment 1 - Event 1

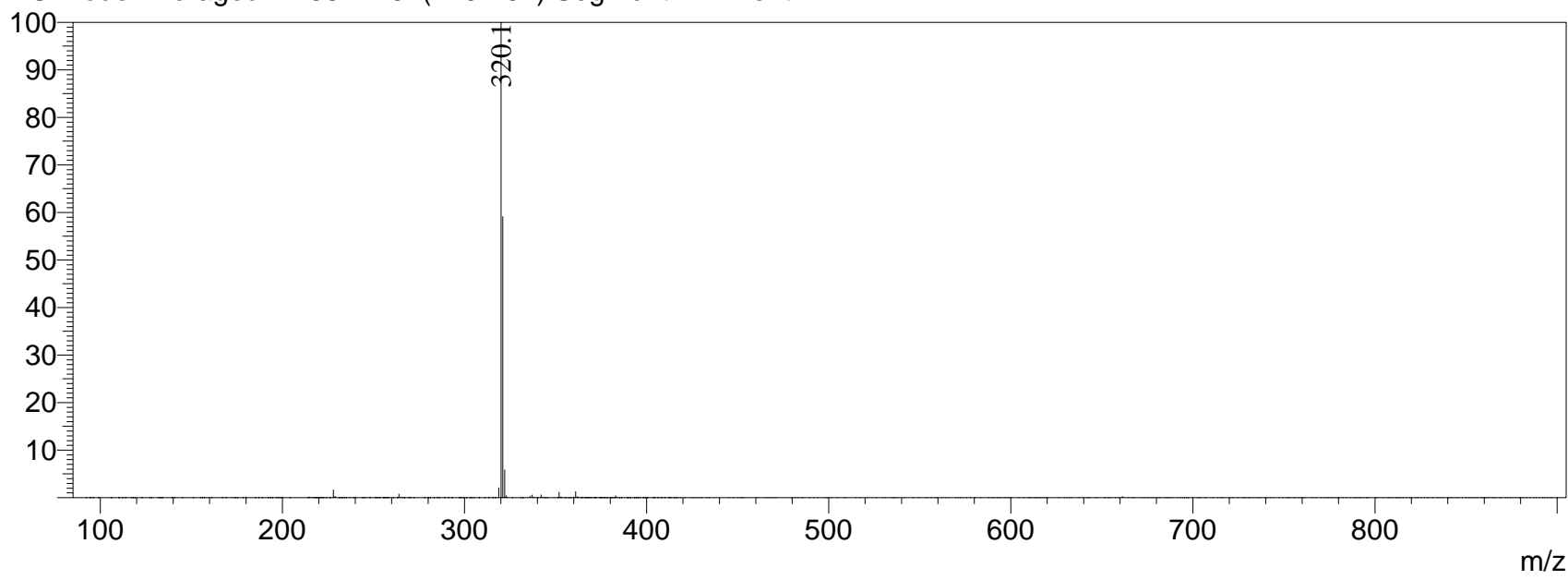




\section{LCMS Analysis Report}

\author{
Acquired by \\ Sample Name \\ Injection Volume \\ Data File \\ Method File \\ Report Format File \\ Date Acquired \\ Comment
}

\author{
: System Administrator \\ : LCMS41-PH-CYT-A-638-0-2(55446-143A1)1T \\ $: 0.5$ \\ : LCMS41-PH-CYT-A-638-0-2(55446-143A1)1T.Icd \\ : ACN-Water-0.1\%FA-10\%B-1.5-3.0MIN(90-900).Icm \\ : LCMS2020-PDA+ELS+TIC+MS.Isr \\ : 2015-10-20 11:11:31 \\ : Mobile phase A:Water/0.1\% FA \\ Mobile phase B:Acetonitrile/0.1\% FA
}

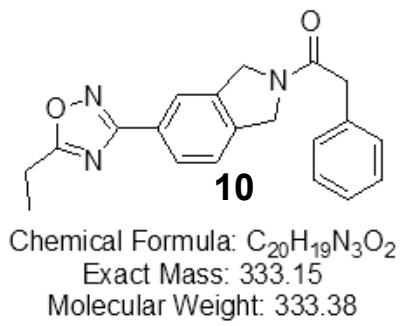

Molecular Weight: 333.38
Instrument Name:Shimadzu LCMS-2020

$<<$ Pump $>>$

Mode

Pump A

Pump B

Total Flow

B Conc.

$<<$ Oven $>>$

Oven Temperature

$<<$ PDA $>>$

PDA Model

Lamp

Start Wavelength

End Wavelength
: Binary gradient

: LC-30AD

: LC-30AD

: $1.5000 \mathrm{~mL} / \mathrm{min}$

: $10.0 \%$

$: 40 \mathrm{C}$
: SPD-M20A
$: \mathrm{D} 2$
$: 190 \mathrm{~nm}$
$: 400 \mathrm{~nm}$

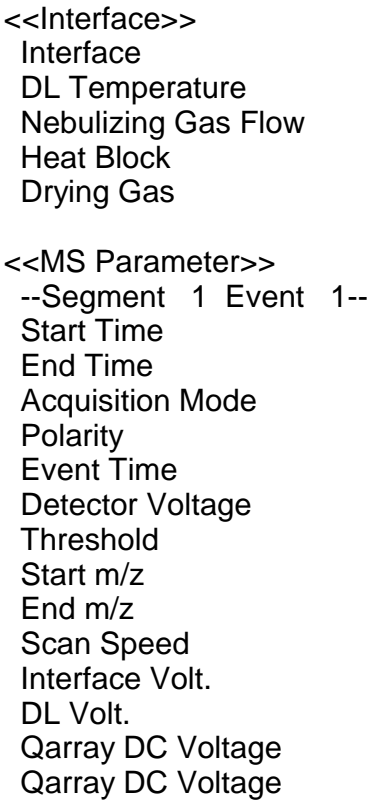

\section{:ESI}

:250 C

$: 1.50 \mathrm{~L} / \mathrm{min}$

:280 C

:On

$15.00 \mathrm{~L} / \mathrm{min}$

$: 0.00 \mathrm{~min}$

:3.00 min

:Scan

:Positive

$: 1.00 \mathrm{sec}$

:+1.10 kV

$: 0$

$: 90.00$

:900.00

:834 u/sec

:Use the Data in the Tuning File

:Use the Data in the Tuning File

:Use the Data in the Tuning File

:Use the Data in the Tuning File
System Configuration

$<<$ Column $>>$

Column Name

Length

Internal Diameter

Description
: Kinetex 2.6um XB-C18 100A

: $50 \mathrm{~mm}$

: $3.0 \mathrm{~mm}$

: 2.6 um

$<<$ LC Time Program $>>$

Time

0.01

2.00

2.60

2.70

3.00
Module

Pumps

Pumps

Pumps

Pumps

Controller
Command

Pump B Conc.

Pump B Conc.

Pump B Conc.

Pump B Conc.

Stop
Value

10

100

100

10 


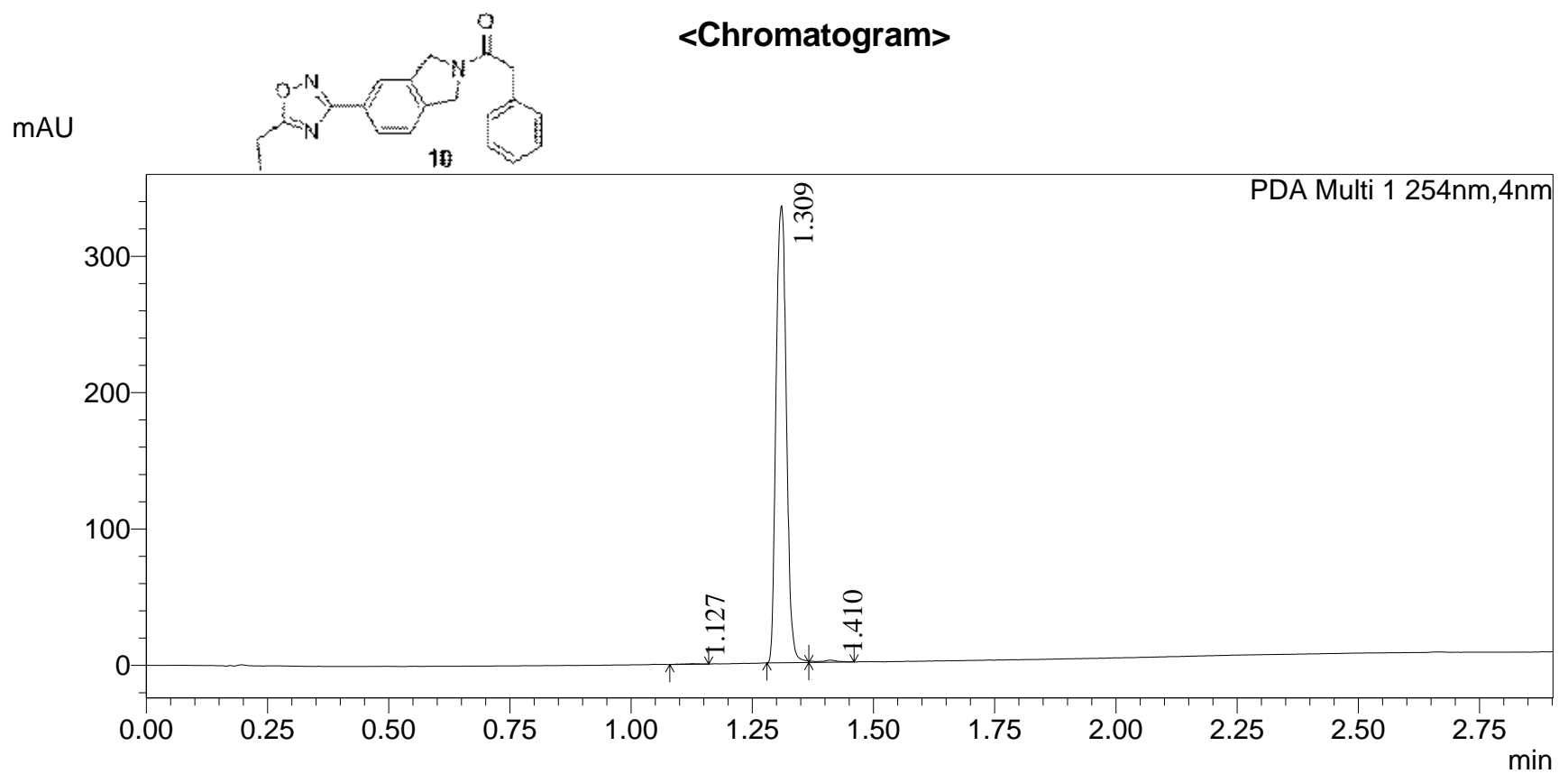

Peak Table

PDA Ch1 254nm

\begin{tabular}{|r|r|r|r|r|r|}
\hline Peak\# & \multicolumn{1}{|c|}{ Ret. Time } & \multicolumn{1}{c|}{ Height } & Height\% & \multicolumn{1}{c|}{ Area } & \multicolumn{1}{c|}{ Area\% } \\
\hline 1 & 1.127 & 371 & 0.112 & 1042 & 0.207 \\
\hline 2 & 1.309 & 330546 & 99.465 & 497294 & 99.053 \\
\hline 3 & 1.410 & 1407 & 0.423 & 3713 & 0.739 \\
\hline Total & & 332324 & 100.000 & 502048 & 100.000 \\
\hline
\end{tabular}

$\mathrm{mV}$

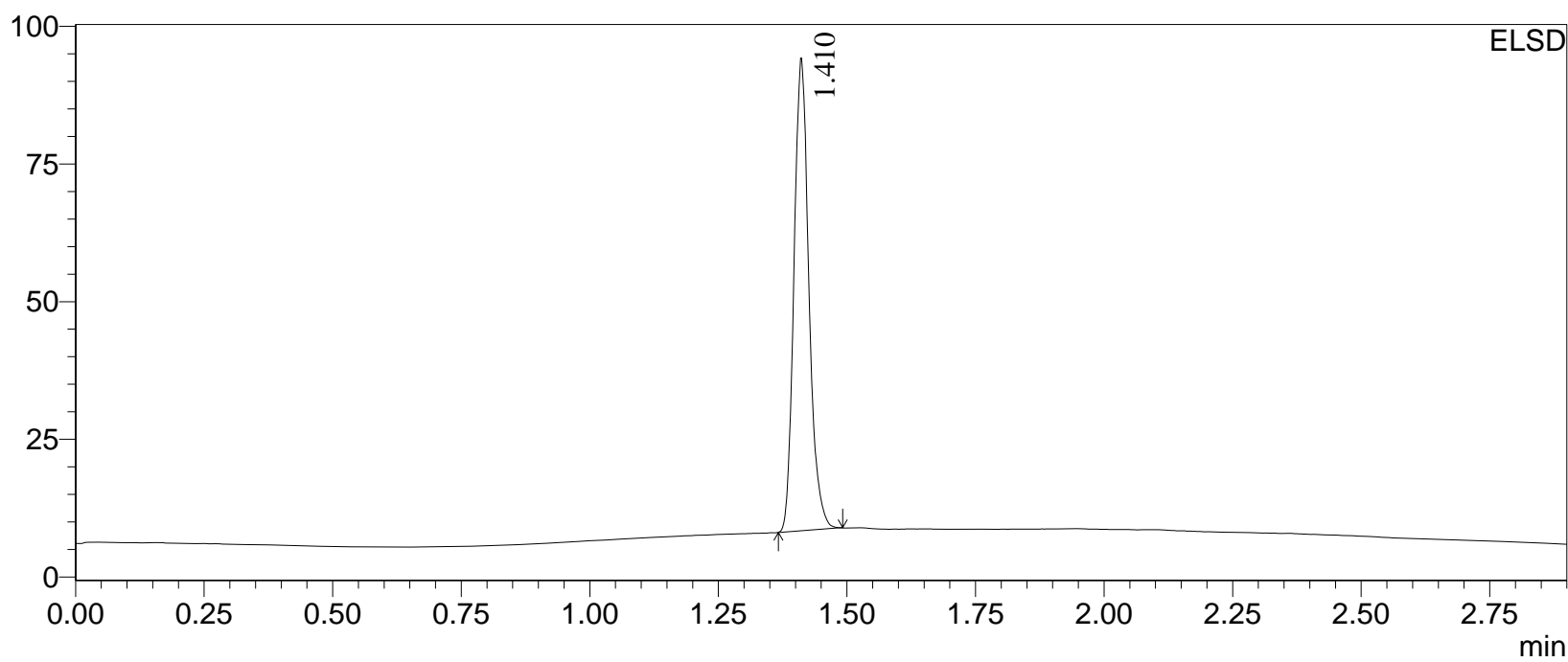

Peak Table

ELSD
\begin{tabular}{|r|r|r|r|r|r|}
\hline Peak\# & Ret. Time & \multicolumn{1}{l|}{ Height } & Height $\%$ & \multicolumn{1}{c|}{ Area } & \multicolumn{1}{c|}{ Area $\%$} \\
\hline 1 & 1.410 & 83235 & 100.000 & 173552 & 100.000 \\
\hline Total & & 83235 & 100.000 & 173552 & 100.000 \\
\hline
\end{tabular}


Line\#:1 R.Time:1.308(Scan\#:83)

Mass Spectrum

MassPeaks:422

Spectrum Mode:Single 1.308(83) BasePeak:334.00(2263690)

BG Mode:Averaged 1.208-1.475(77-93) Segment 1 - Event 1

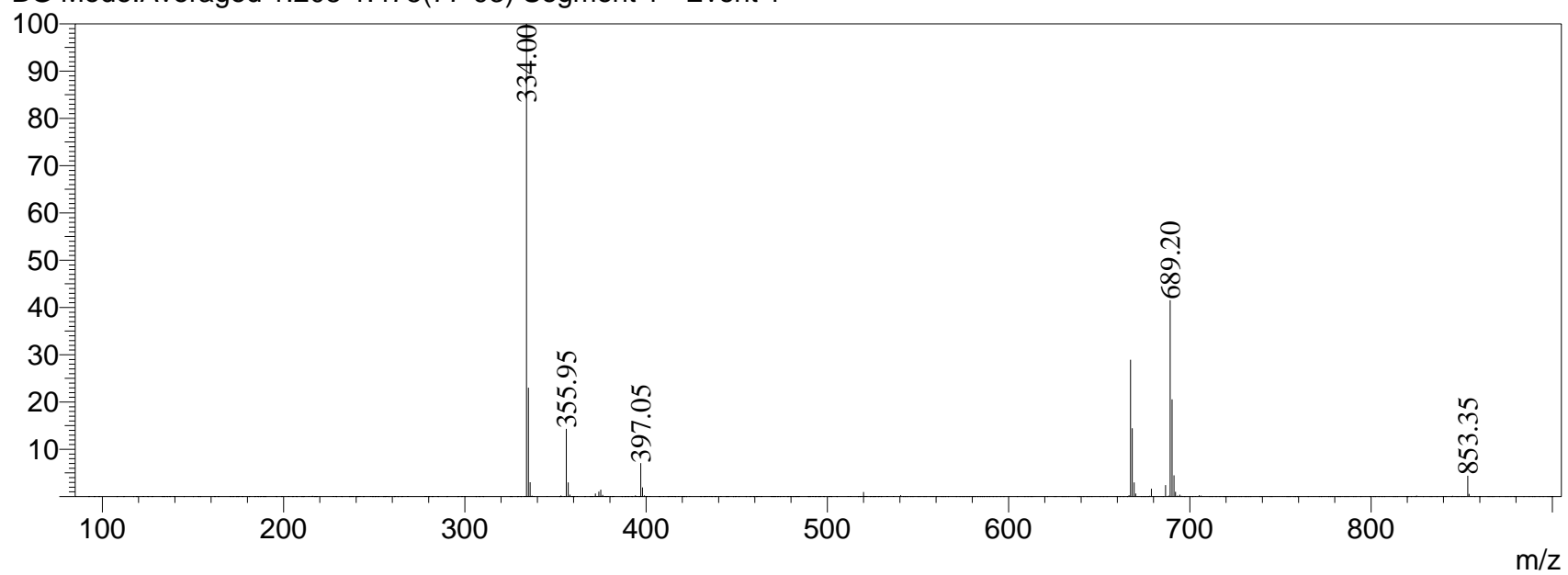




\section{LCMS Analysis Report}

Acquired by

Sample Name

Injection Volume

Data File

Method File

Report Format File

Date Acquired

Comment
: System Administrator

: LCMS42-PH-CYT-A-635-0-2(55460-129D1)1T

$: 20$

: LCMS42-PH-CYT-A-635-0-2(55460-129D1)1T.Icd

: ACN-Water-ACN-10\%B-1.5-3.6MIN(90-900).Icm

: LCMS2020-PDA+ELS+TIC+MS.Isr

: 2015-10-19 13:11:57

: Mobile Phase A:Water/5mM NH4HCO3 Mobile phase B:ACN

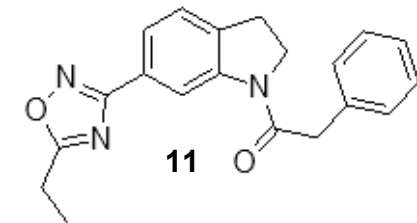

Chemical Formula: $\mathrm{C}_{20} \mathrm{H}_{19} \mathrm{~N}_{3} \mathrm{O}_{2}$

Exact Mass: 333.15

Molecular Weight: 333.38
Instrument Name:Shimadzu LCMS-2020

$<<$ Pump $>>$

Mode

Pump A

Pump B

Total Flow

B Conc.

$<<$ Oven $>>$

Oven Temperature

$: 35 \mathrm{C}$

$<<$ PDA $>>$

PDA Model

Lamp

Start Wavelength

End Wavelength

System Configuration

$<<$ Column $>>$

Column Name

Length

Internal Diameter

Description

XBridge $\mathrm{C} 18$

: LC-20AD

: LC-20AD

: $1.5000 \mathrm{~mL} / \mathrm{min}$

: $10.0 \%$

: SPD-M20A

: D2

: $190 \mathrm{~nm}$

: $400 \mathrm{~nm}$

: $50 \mathrm{~mm}$

$: 4.6 \mathrm{~mm}$

$: 3.5 \mu \mathrm{m}$
$<<$ Interface >>

Interface :ESI

DL Temperature $\quad: 250 \mathrm{C}$

Nebulizing Gas Flow $\quad: 1.50 \mathrm{~L} / \mathrm{min}$

Heat Block $\quad: 250 \mathrm{C}$

Drying Gas :On

$15.00 \mathrm{~L} / \mathrm{min}$

<<MS Parameter >>

--Segment 1 Event 1--

Start Time $\quad: 0.00 \mathrm{~min}$

End Time $\quad: 3.60 \mathrm{~min}$

Acquisition Mode :Scan

Polarity :Positive

Event Time $\quad: 0.50 \mathrm{sec}$

Detector Voltage $\quad:+0.85 \mathrm{kV}$

Threshold $: 0$

Start $\mathrm{m} / \mathrm{z} \quad: 90.00$

End $\mathrm{m} / \mathrm{z} \quad: 900.00$

Scan Speed $\quad: 1667 \mathrm{u} / \mathrm{sec}$

Interface Volt.

DL Volt.

Qarray DC Voltage

Qarray DC Voltage

:Use the Data in the Tuning File

:Use the Data in the Tuning File

:Use the Data in the Tuning File

:Use the Data in the Tuning File

$<<$ LC Time Program $>>$

$\begin{array}{ll}\text { Time } & \text { Module } \\ 0.01 & \text { Pumps } \\ 2.20 & \text { Pumps } \\ 3.20 & \text { Pumps } \\ 3.30 & \text { Pumps } \\ 3.60 & \text { Controller }\end{array}$

$\begin{array}{ll}\text { Command } & \text { Value } \\ \text { Pump B Conc. } & 10 \\ \text { Pump B Conc. } & 95 \\ \text { Pump B Conc. } & 95 \\ \text { Pump B Conc. } & 10\end{array}$

Stop 


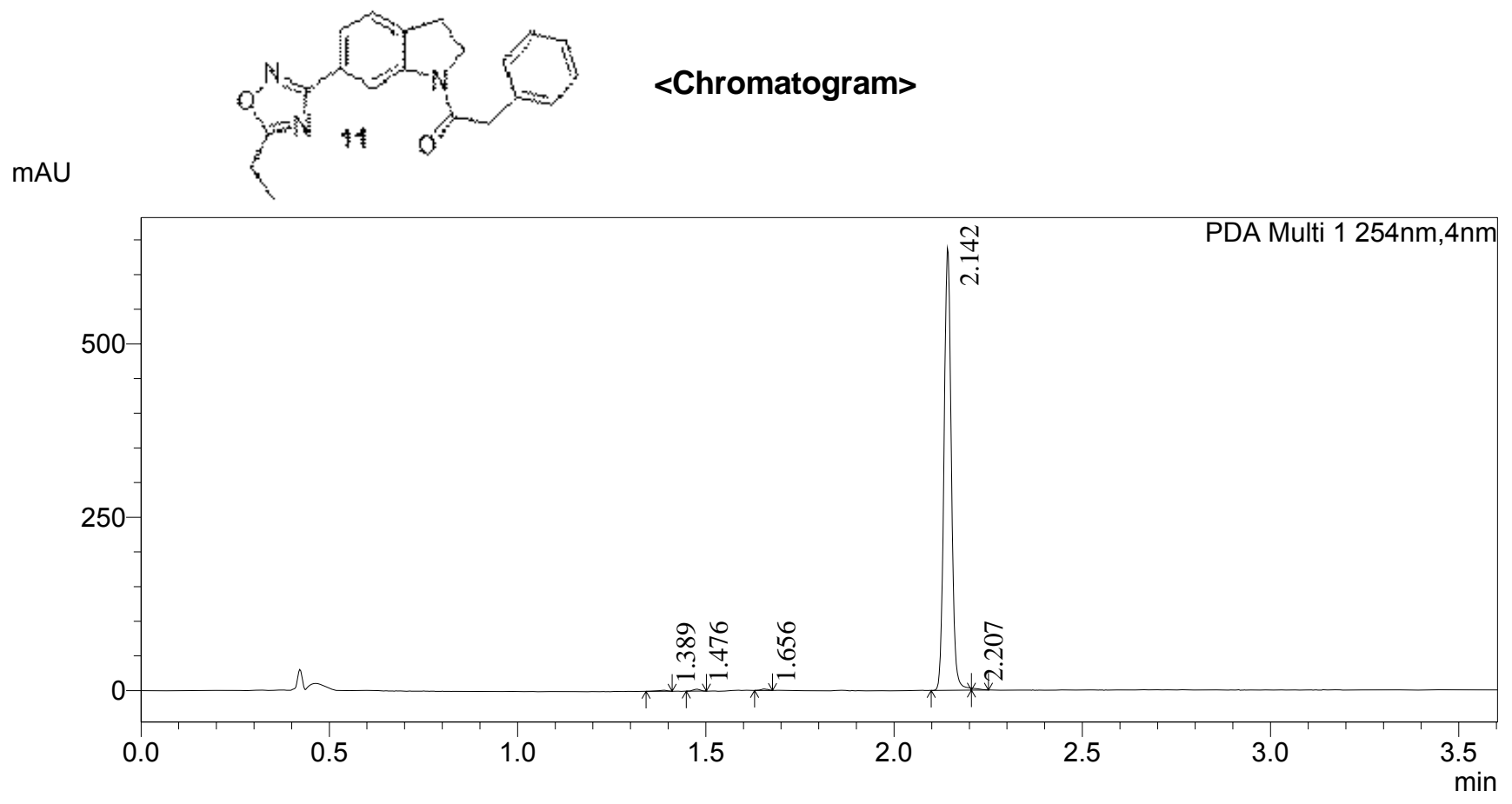

Peak Table

PDA Ch1 254nm

\begin{tabular}{|r|r|r|r|r|r|}
\hline Peak\# & Ret. Time & \multicolumn{1}{c|}{ Height } & Height\% & \multicolumn{1}{c|}{ Area } & \multicolumn{1}{c|}{ Area\% } \\
\hline 1 & 1.389 & 1559 & 0.241 & 2302 & 0.263 \\
\hline 2 & 1.476 & 2744 & 0.424 & 3618 & 0.414 \\
\hline 3 & 1.656 & 2123 & 0.328 & 2561 & 0.293 \\
\hline 4 & 2.142 & 638024 & 98.572 & 862496 & 98.627 \\
\hline 5 & 2.207 & 2818 & 0.435 & 3527 & 0.403 \\
\hline Total & & 647269 & 100.000 & 874502 & 100.000 \\
\hline
\end{tabular}

mAU

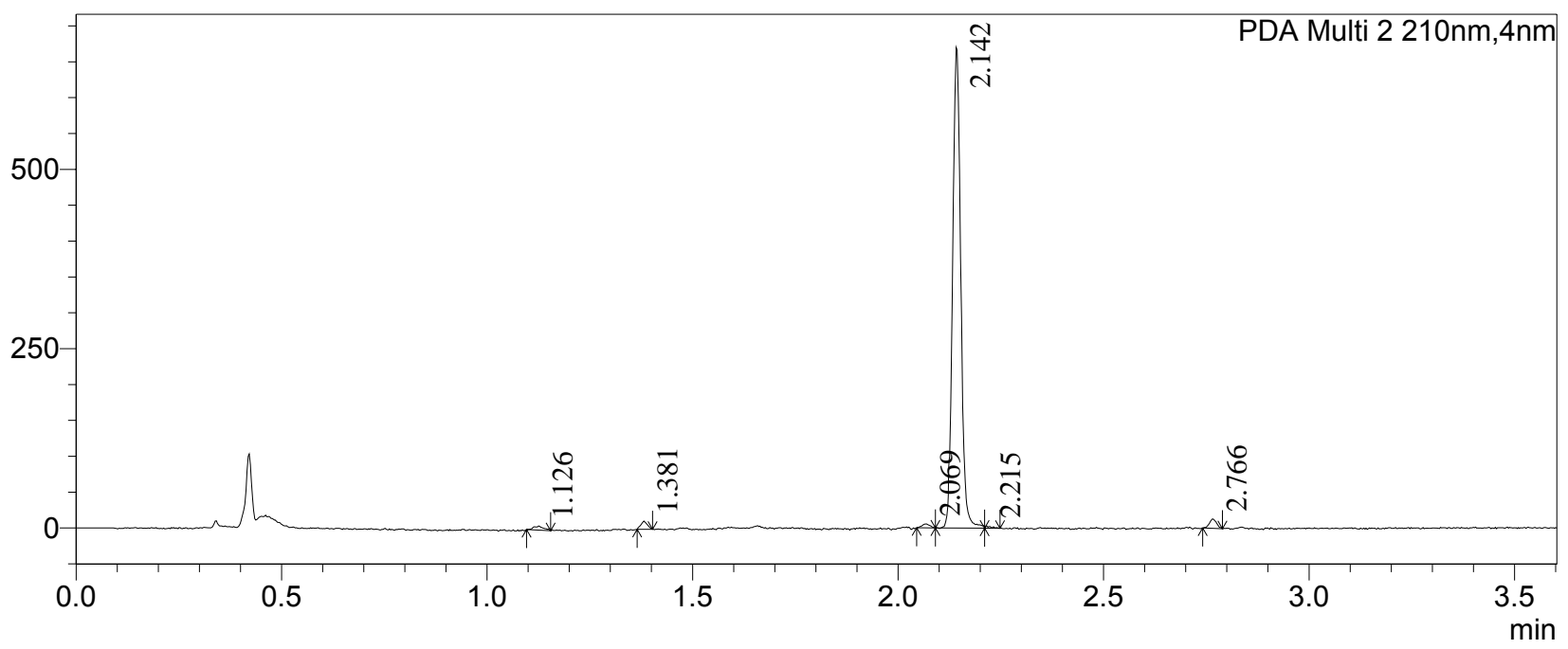

Peak Table

PDA Ch2 210nm

\begin{tabular}{|r|r|r|r|r|r|}
\hline Peak\# & \multicolumn{1}{|c|}{ Ret. Time } & \multicolumn{1}{c|}{ Height } & Height\% & \multicolumn{1}{c|}{ Area } & \multicolumn{1}{c|}{ Area } \\
\hline 1 & 1.126 & 5802 & 0.818 & 9338 & 0.959 \\
\hline 2 & 1.381 & 11652 & 1.643 & 12426 & 1.277 \\
\hline 3 & 2.069 & 5384 & 0.759 & 6936 & 0.713 \\
\hline 4 & 2.142 & 670086 & 94.471 & 925764 & 95.116 \\
\hline 5 & 2.215 & 3173 & 0.447 & 3070 & 0.315 \\
\hline 6 & 2.766 & 13208 & 1.862 & 15764 & 1.620 \\
\hline Total & & 709306 & 100.000 & 973297 & 100.000 \\
\hline
\end{tabular}


$\mathrm{mV}$

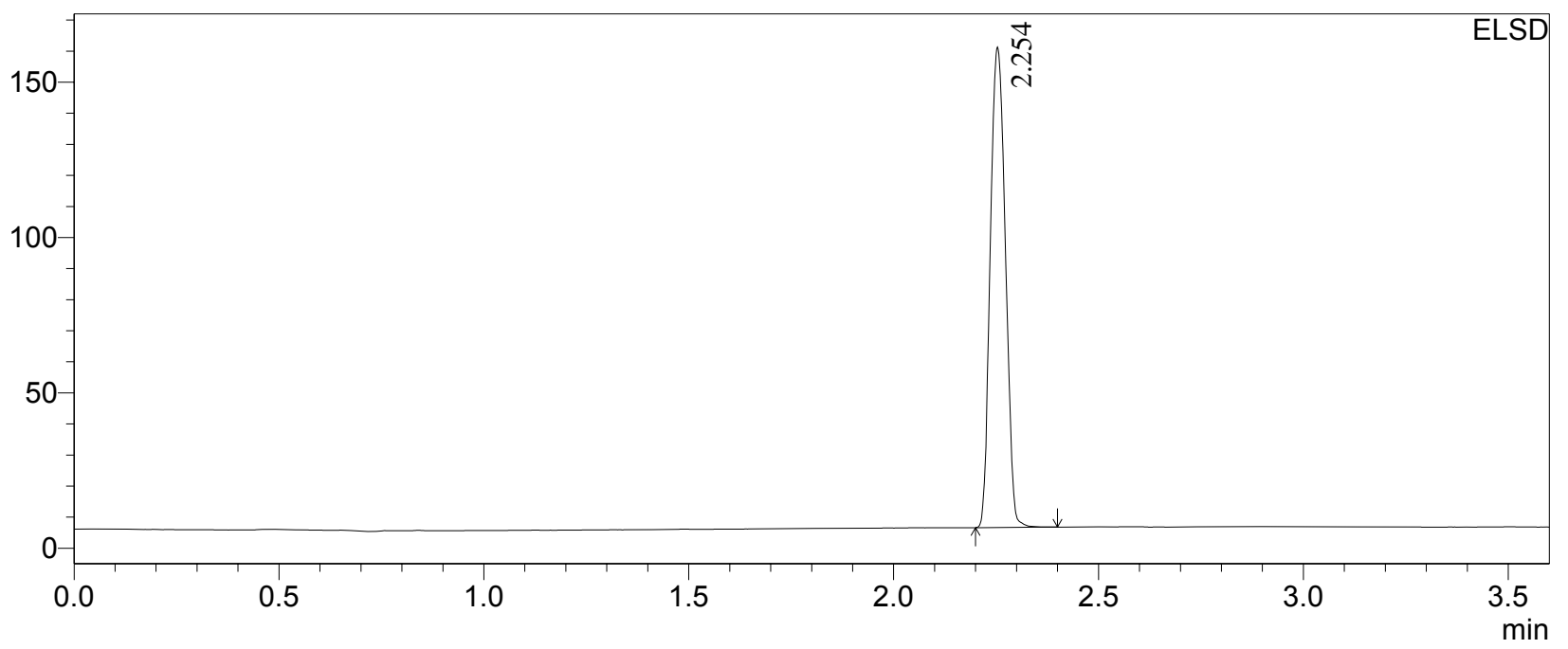

Peak Table

ELSD

\begin{tabular}{|r|r|r|r|r|r|}
\hline Peak\# & Ret. Time & Height & \multicolumn{1}{c|}{ Height\% } & \multicolumn{1}{c|}{ Area } & \multicolumn{1}{c|}{ Area\% } \\
\hline 1 & 2.254 & 152934 & 100.000 & 397758 & 100.000 \\
\hline Total & & 152934 & 100.000 & 397758 & 100.000 \\
\hline
\end{tabular}

Line\#:1 R.Time:2.144(Scan\#:262)

Mass Spectrum

MassPeaks:400

Spectrum Mode:Averaged 2.135-2.152(261-263) BasePeak:334.05(730631)

BG Mode:Calc Segment 1 - Event 1

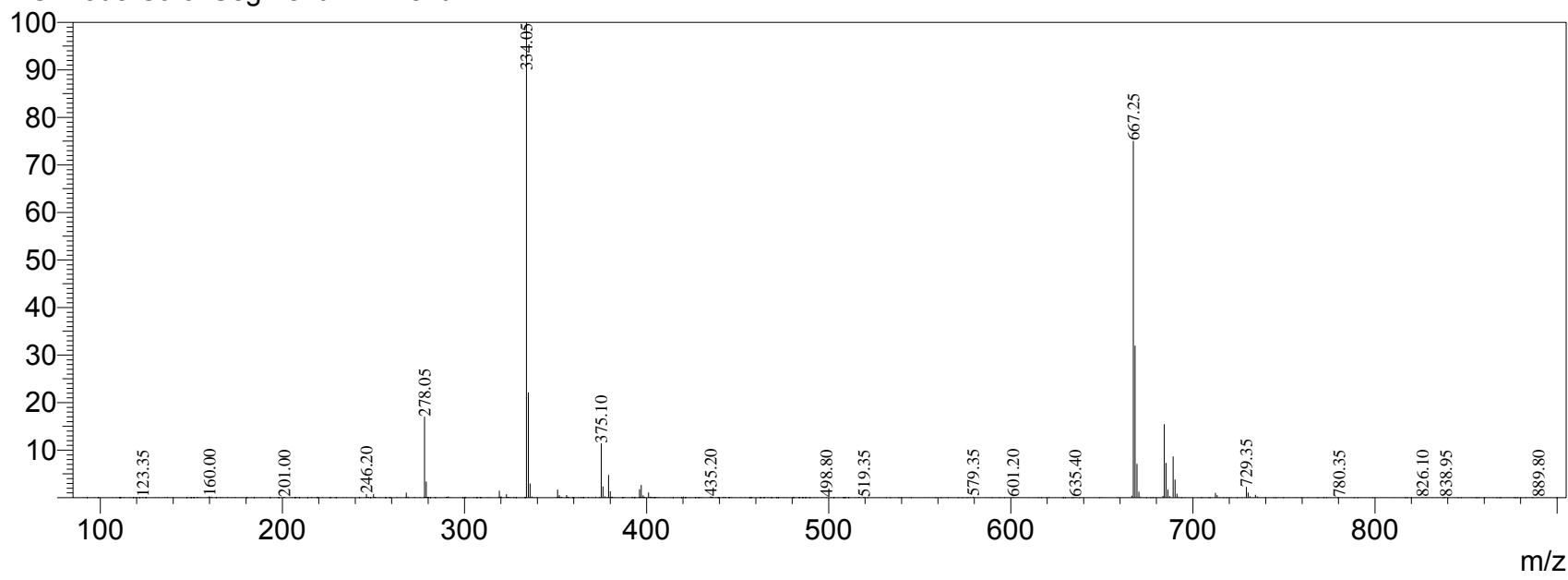




\section{LCMS Analysis Report}

\author{
Acquired by \\ Sample Name \\ Injection Volume \\ Data File \\ Method File \\ Report Format File \\ Date Acquired \\ Comment
}

: System Administrator

: LCMS20-PH-CYT-A-639-0-2(55448-172A1)1T

$: 1$

: LCMS20-PH-CYT-A-639-0-2(55448-172A1)1T001.Icd

: ACN-Water-0.05\%TFA-5\%B-70\%-5.6MIN(90-900).Icm

: LCMS2020-PDA(DW)+ELSD+MS.Isr

: 2015-10-9 14:52:11

: Mobile Phase A:Water/0.05\%TFA;

Mobile Phase B:Acetonitrile/0.05\%TFA;

Instrument Name:Shimadzu LCMS-2020

$<<$ Pump $>>$

Mode

: Binary gradient

Pump A

Pump B

Total Flow

: LC-20AD

B Conc.

: LC-20AD

: $1.0000 \mathrm{~mL} / \mathrm{min}$

: $5.0 \%$

$<<$ Oven $>>$

Oven Temperature

$: 40 \mathrm{C}$

$<<$ PDA $>>$

PDA Model

Lamp

SPD-M20A

: D2

Start Wavelength

$190 \mathrm{~nm}$

End Wavelength

: $400 \mathrm{~nm}$

System Configuration

<<Interface>>
Interface
DL Temperature
Nebulizing Gas Flow
Heat Block
Drying Gas
<<MS Parameter>>
--Segment 1 Event 1--
Start Time
End Time
Acquisition Mode
Polarity
Event Time
Detector Voltage
Threshold
Start m/z
End m/z
Scan Speed
Interface Volt.
DL Volt.
Qarray DC Voltage
Qarray DC Voltage

$<<$ Interface $>>$

DL Temperature

Nebulizing Gas Flow

Heat Block

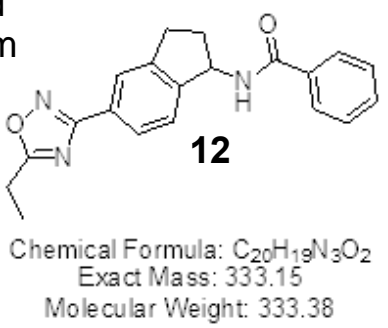

:ESI

$: 250 \mathrm{C}$

$: 1.50 \mathrm{~L} / \mathrm{min}$

$: 200 \mathrm{C}$

:On

$15.00 \mathrm{~L} / \mathrm{min}$

$: 0.00 \mathrm{~min}$

$: 5.60 \mathrm{~min}$

:Scan

:Positive

:0.60 sec

:+1.05 kV

$: 0$

$: 90.00$

:900.00

:1364 u/sec

:Use the Data in the Tuning File

:Use the Data in the Tuning File

:Use the Data in the Tuning File

:Use the Data in the Tuning File

$<<$ LC Time Program $>>$

$\begin{array}{ll}\text { Time } & \text { Module } \\ 0.01 & \text { Pumps } \\ 4.00 & \text { Pumps } \\ 5.20 & \text { Pumps } \\ 5.30 & \text { Pumps } \\ 5.60 & \text { Controller }\end{array}$

$\begin{array}{ll}\text { Command } & \text { Value } \\ \text { Pump B Conc. } & 5 \\ \text { Pump B Conc. } & 70 \\ \text { Pump B Conc. } & 70 \\ \text { Pump B Conc. } & 5\end{array}$

Stop 
mAU
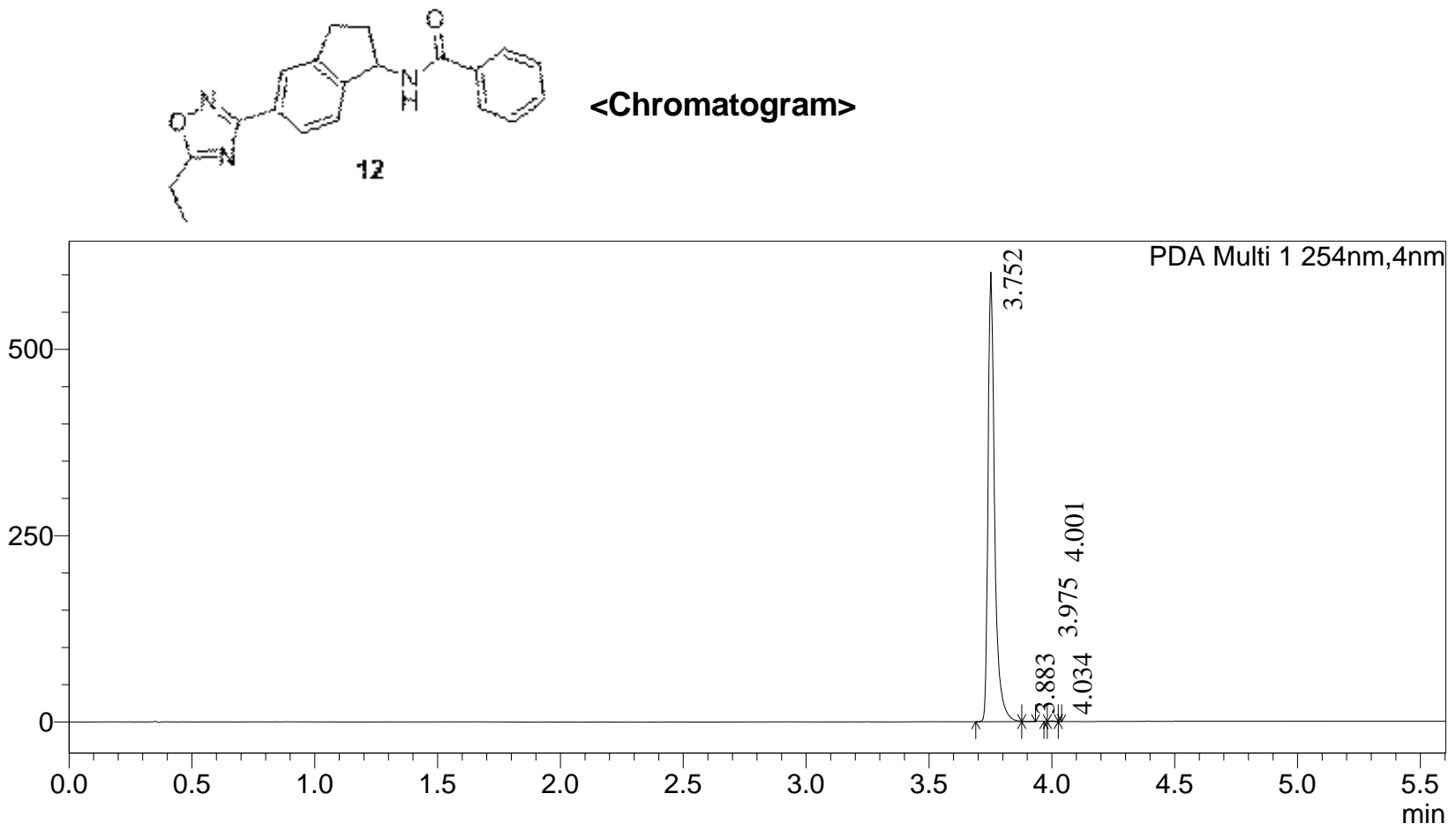

Peak Table

PDA Ch1 254nm

\begin{tabular}{|r|r|r|r|r|r|}
\hline Peak\# & Ret. Time & \multicolumn{1}{|c|}{ Height } & Height\% & Area & \multicolumn{1}{c|}{ Area\% } \\
\hline 1 & 3.752 & 603679 & 99.748 & 1133169 & 99.815 \\
\hline 2 & 3.883 & 379 & 0.063 & 503 & 0.044 \\
\hline 3 & 3.975 & 52 & 0.009 & 46 & 0.004 \\
\hline 4 & 4.001 & 943 & 0.156 & 1473 & 0.130 \\
\hline 5 & 4.034 & 153 & 0.025 & 80 & 0.007 \\
\hline Total & & 605206 & 100.000 & 1135272 & 100.000 \\
\hline
\end{tabular}

mAU

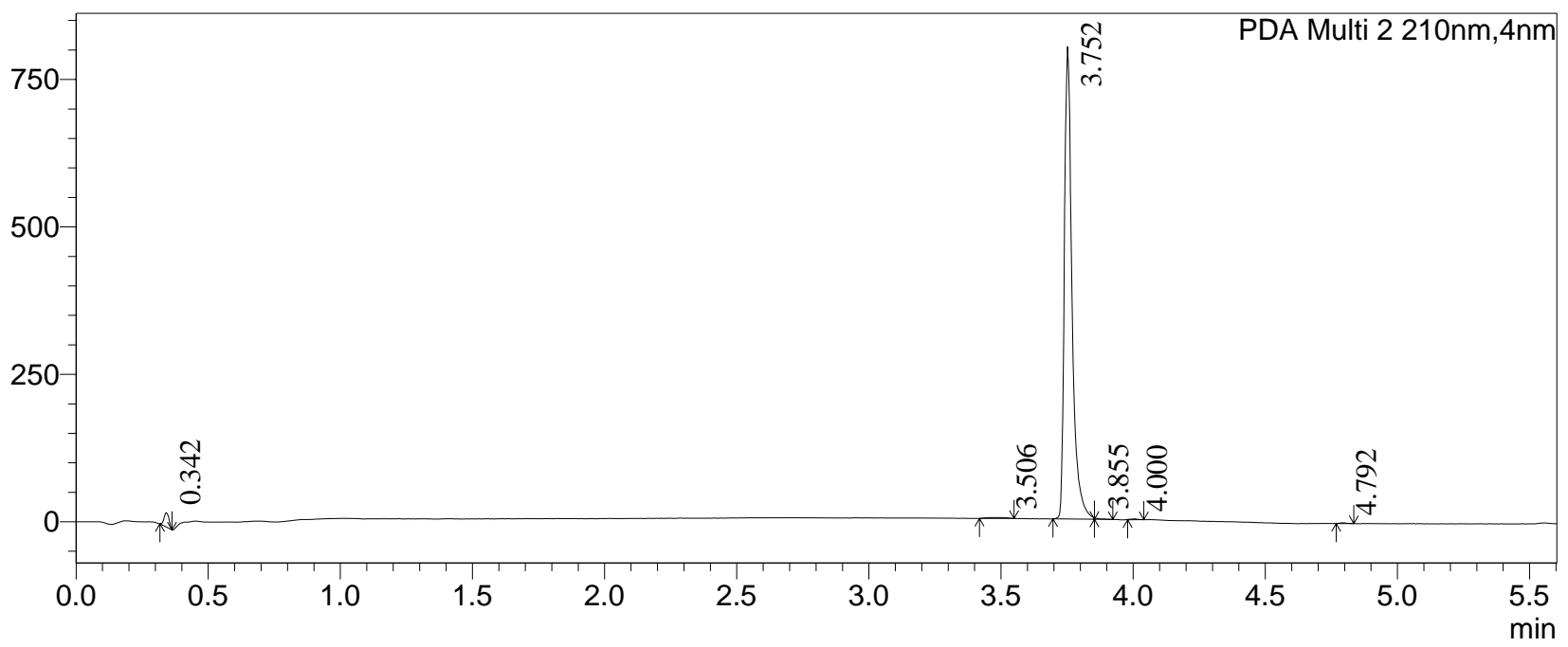

Peak Table

PDA Ch2 210nm

\begin{tabular}{|r|r|r|r|r|r|}
\hline Peak\# & \multicolumn{1}{|c|}{ Ret. Time } & \multicolumn{1}{c|}{ Height } & Height\% & \multicolumn{1}{c|}{ Area } & \multicolumn{1}{c|}{ Area\% } \\
\hline 1 & 0.342 & 24397 & 2.930 & 31081 & 1.863 \\
\hline 2 & 3.506 & 1768 & 0.212 & 8073 & 0.484 \\
\hline 3 & 3.752 & 801314 & 96.224 & 1621628 & 97.210 \\
\hline 4 & 3.855 & 2258 & 0.271 & 2455 & 0.147 \\
\hline 5 & 4.000 & 1607 & 0.193 & 2571 & 0.154 \\
\hline 6 & 4.792 & 1417 & 0.170 & 2370 & 0.142 \\
\hline Total & & 832761 & 100.000 & 1668178 & 100.000 \\
\hline
\end{tabular}


$\mathrm{mV}$

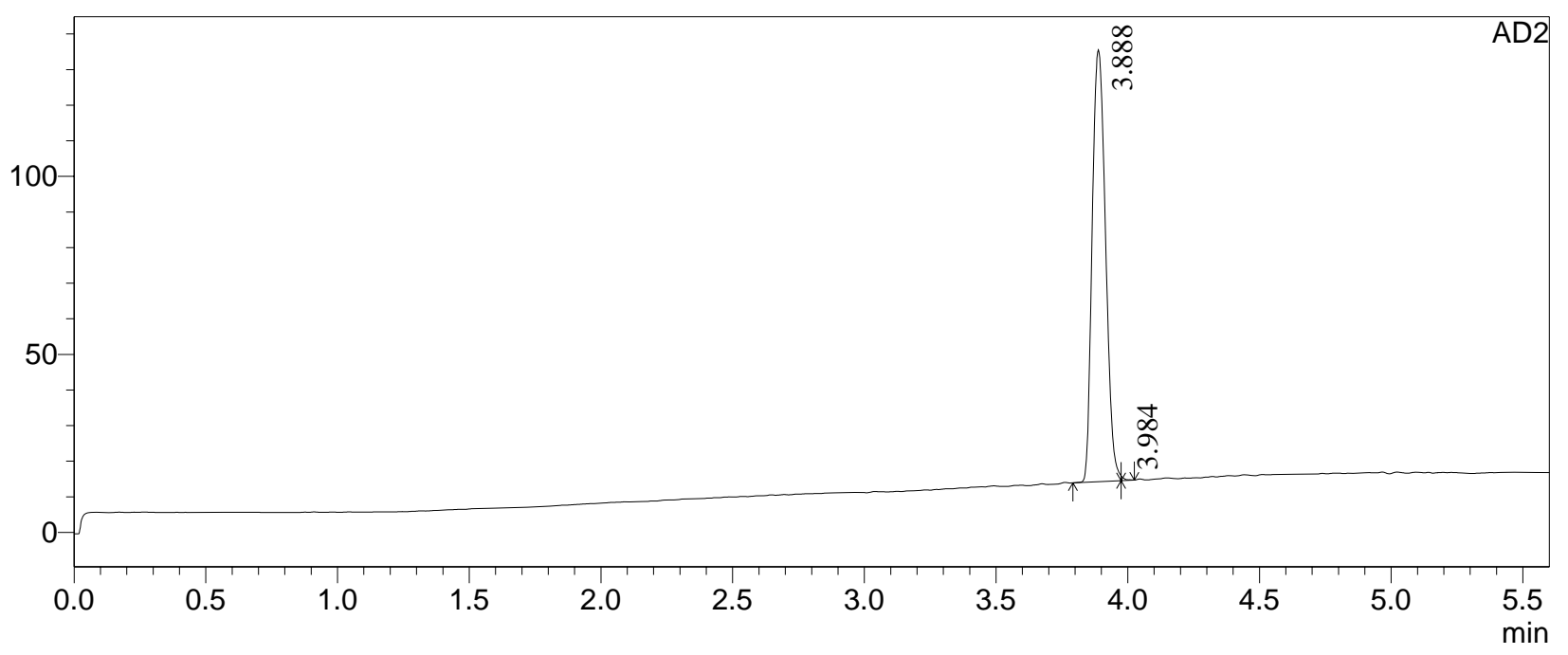

Peak Table

AD2
\begin{tabular}{|r|r|r|r|r|r|}
\hline Peak\# & Ret. Time & \multicolumn{1}{c|}{ Height } & Height $\%$ & \multicolumn{1}{c|}{ Area } & \multicolumn{1}{c|}{ Area $\%$} \\
\hline 1 & 3.888 & 120792 & 99.255 & 427569 & 99.785 \\
\hline 2 & 3.984 & 907 & 0.745 & 921 & 0.215 \\
\hline Total & & 121699 & 100.000 & 428490 & 100.000 \\
\hline
\end{tabular}

Line\#:1 R.Time:3.744(Scan\#:380)

Mass Spectrum

MassPeaks:368

Spectrum Mode:Single 3.744(380) BasePeak:688.9(640778)

BG Mode:Averaged 3.674-3.844(373-390) Segment 1 - Event 1

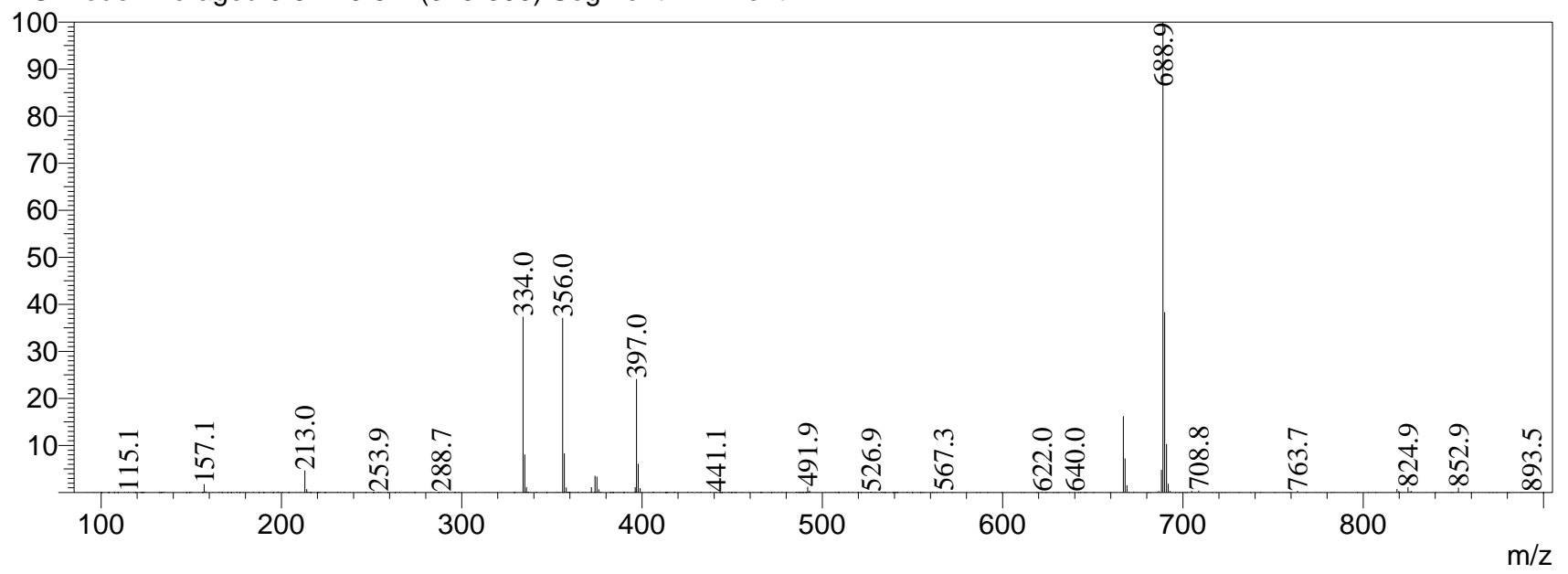




\section{LCMS Analysis Report}

\author{
Acquired by \\ Sample Name \\ Injection Volume \\ Data File \\ Method File \\ Report Format File \\ Date Acquired \\ Comment
}

: System Administrator

: LCMS17-PH-CYT-A-699-0-2(57213-071A1)1T

$: 1$

: LCMS17-PH-CYT-A-699-0-2(57213-071A1)1T001.Icd

: ACN-Water-0.05\%TFA-5\%B-70\%-1.0-5.6MIN(90-900).Icm

: LCMS2020-PDA(DW)-ELSD-MS-.Isr

: 2015-11-13 12:58:31

: Mobile Phase A:Water/0.05\%TFA;

Mobile Phase B:Acetonitrile/0.05\%TFA;

Instrument Name:Shimadzu LCMS-2020

$<<$ Pump $>>$

Mode

Pump A

Pump B

Total Flow

B Conc.

: Binary gradient

: LC-20AD

: LC-20AD

: $1.0000 \mathrm{~mL} / \mathrm{min}$

$: 5.0 \%$

$<<$ Oven $>>$

Oven Temperature

: $40 \mathrm{C}$

$<<$ PDA $>$

PDA Model

Lamp

: D2

Start Wavelength

End Wavelength

: $190 \mathrm{~nm}$

: $400 \mathrm{~nm}$

System Configuration

$<<$ Column $>>$

Column Name

Length

Internal Diameter

Description

: Shim-pack XR-ODS

$<<$ Interface $>>$

Interface

DL Temperature

Nebulizing Gas Flow

Heat Block

Drying Gas

$<<$ MS Parameter $>>$

--Segment 1 Event 1--

Start Time

End Time

Acquisition Mode

Polarity

Event Time

Detector Voltage

Threshold

Start $\mathrm{m} / \mathrm{z}$

End $\mathrm{m} / \mathrm{z}$

Scan Speed

Interface Volt.

DL Volt.

Qarray DC Voltage

Qarray DC Voltage

: $50 \mathrm{~mm}$

: $3.0 \mathrm{~mm}$

: 2.2um particle

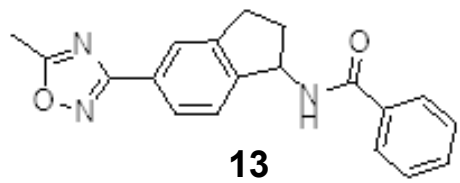

Chemical Formula: $\mathrm{C}_{19} \mathrm{H}_{17} \mathrm{~N}_{3} \mathrm{O}_{2}$

:ESI Exact Mass: 319.13

:250 C Molecular Weight: 319.36

$: 1.50 \mathrm{~L} / \mathrm{min}$

$: 200 \mathrm{C}$

:On

$15.00 \mathrm{~L} / \mathrm{min}$

$: 0.00 \mathrm{~min}$

:5.60 min

:Scan

:Positive

$: 0.50 \mathrm{sec}$

:+1.15 kV

$: 0$

$: 90.00$

:900.00

:1667 u/sec

:Use the Data in the Tuning File

:Use the Data in the Tuning File

:Use the Data in the Tuning File

:Use the Data in the Tuning File

$<<$ LC Time Program $>>$

$\begin{array}{ll}\text { Time } & \text { Module } \\ 0.01 & \text { Pumps } \\ 4.20 & \text { Pumps } \\ 5.20 & \text { Pumps } \\ 5.30 & \text { Pumps } \\ 5.60 & \text { Controller }\end{array}$

$\begin{array}{ll}\text { Command } & \text { Value } \\ \text { Pump B Conc. } & 5 \\ \text { Pump B Conc. } & 70 \\ \text { Pump B Conc. } & 70 \\ \text { Pump B Conc. } & 5\end{array}$

Stop 
mAU
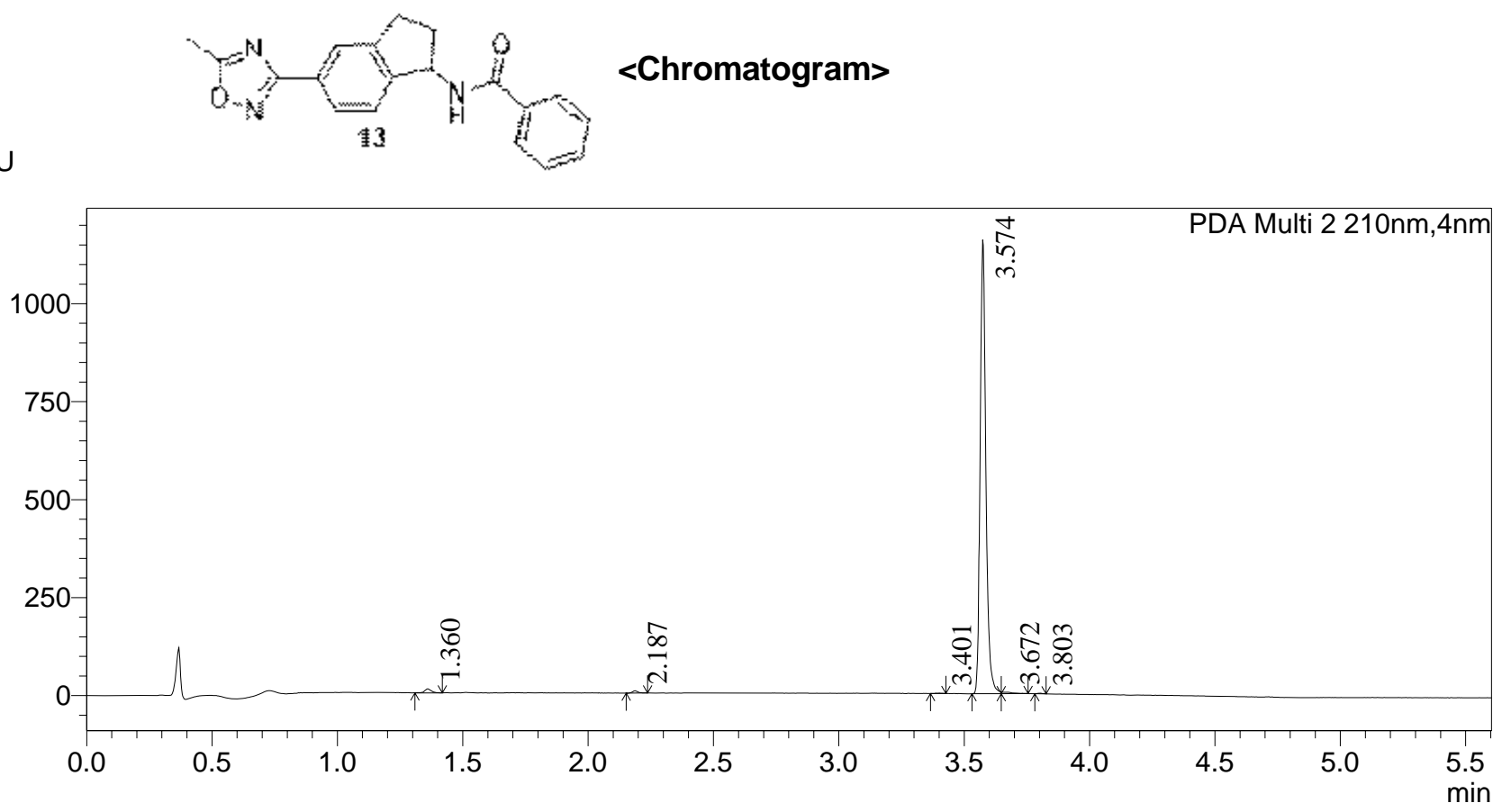

Peak Table

PDA Ch2 210nm

\begin{tabular}{|r|r|r|r|r|r|}
\hline Peak\# & Ret. Time & \multicolumn{1}{|c|}{ Height } & Height $\%$ & \multicolumn{1}{c|}{ Area } & \multicolumn{1}{c|}{ Area\% } \\
\hline 1 & 1.360 & 10330 & 0.875 & 19139 & 1.012 \\
\hline 2 & 2.187 & 5474 & 0.464 & 8444 & 0.447 \\
\hline 3 & 3.401 & 903 & 0.076 & 1326 & 0.070 \\
\hline 4 & 3.574 & 1158367 & 98.145 & 1849418 & 97.800 \\
\hline 5 & 3.672 & 3649 & 0.309 & 10869 & 0.575 \\
\hline 6 & 3.803 & 1536 & 0.130 & 1833 & 0.097 \\
\hline Total & & 1180259 & 100.000 & 1891029 & 100.000 \\
\hline
\end{tabular}

mAU

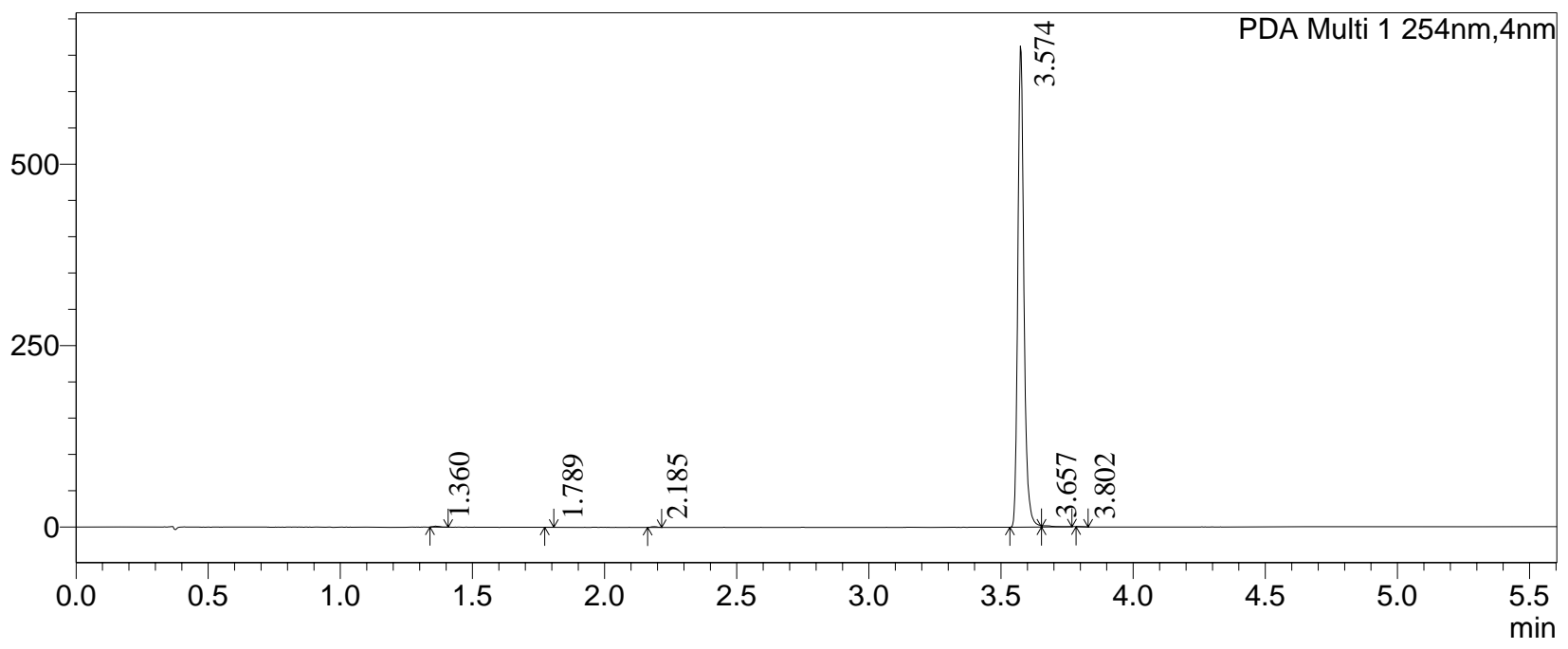

Peak Table

PDA Ch1 254nm

\begin{tabular}{|r|r|r|r|r|r|}
\hline Peak\# & Ret. Time & \multicolumn{1}{|c|}{ Height } & Height\% & Area & \multicolumn{1}{c|}{ Area\% } \\
\hline 1 & 1.360 & 1353 & 0.202 & 2332 & 0.217 \\
\hline 2 & 1.789 & 269 & 0.040 & 253 & 0.024 \\
\hline 3 & 2.185 & 1039 & 0.156 & 1466 & 0.136 \\
\hline 4 & 3.574 & 663191 & 99.243 & 1066749 & 99.088 \\
\hline 5 & 3.657 & 1769 & 0.265 & 4898 & 0.455 \\
\hline 6 & 3.802 & 625 & 0.094 & 872 & 0.081 \\
\hline Total & & 668247 & 100.000 & 1076571 & 100.000 \\
\hline
\end{tabular}


$\mathrm{mV}$

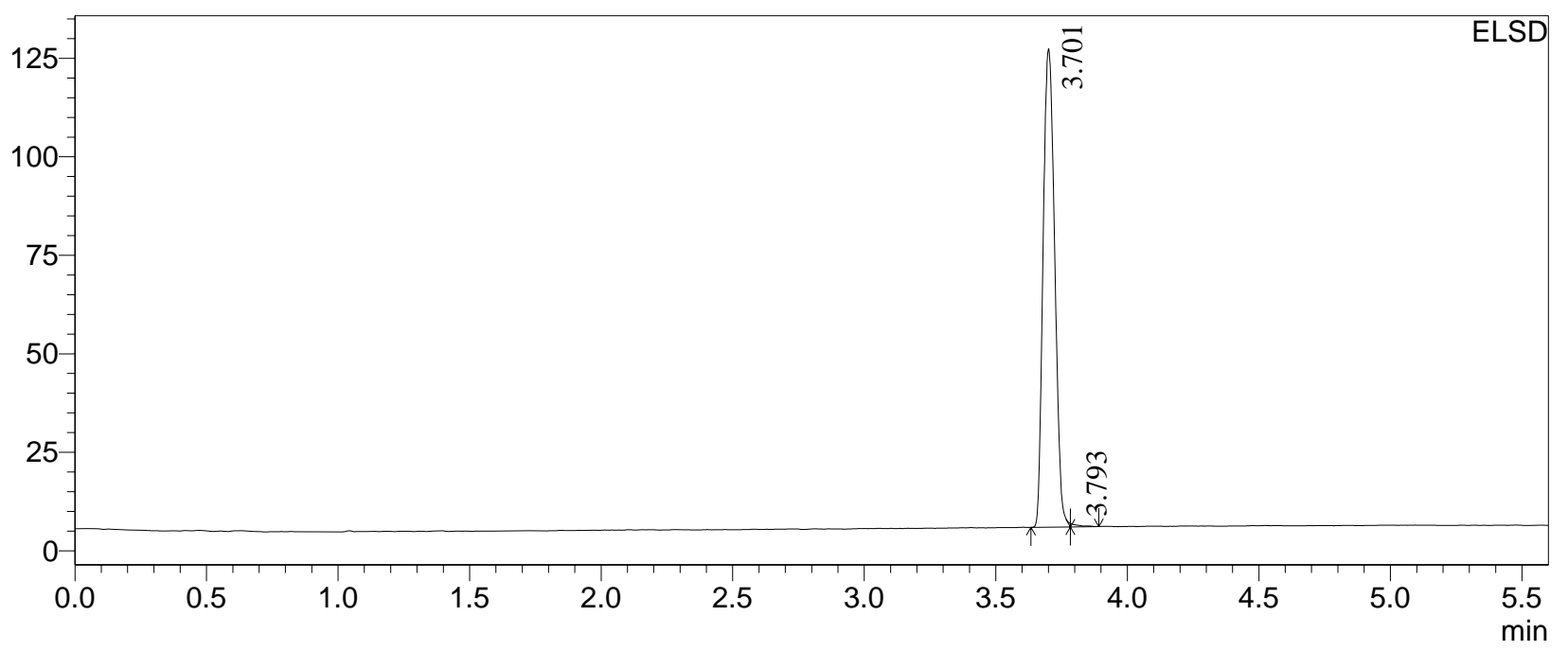

Peak Table

ELSD

\begin{tabular}{|r|r|r|r|r|r|}
\hline Peak\# & Ret. Time & \multicolumn{1}{|c|}{ Height } & \multicolumn{1}{c|}{ Height\% } & \multicolumn{1}{c|}{ Area } & \multicolumn{1}{c|}{ Area $\%$} \\
\hline 1 & 3.701 & 118900 & 99.422 & 372438 & 99.557 \\
\hline 2 & 3.793 & 692 & 0.578 & 1657 & 0.443 \\
\hline Total & & 119592 & 100.000 & 374095 & 100.000 \\
\hline
\end{tabular}

Line\#:1 R.Time:3.560(Scan\#:437)

Mass Spectrum

MassPeaks:372

Spectrum Mode:Single 3.560(437) BasePeak:320.1(126123)

BG Mode:Peak Start 3.494(429) Segment 1 - Event 1

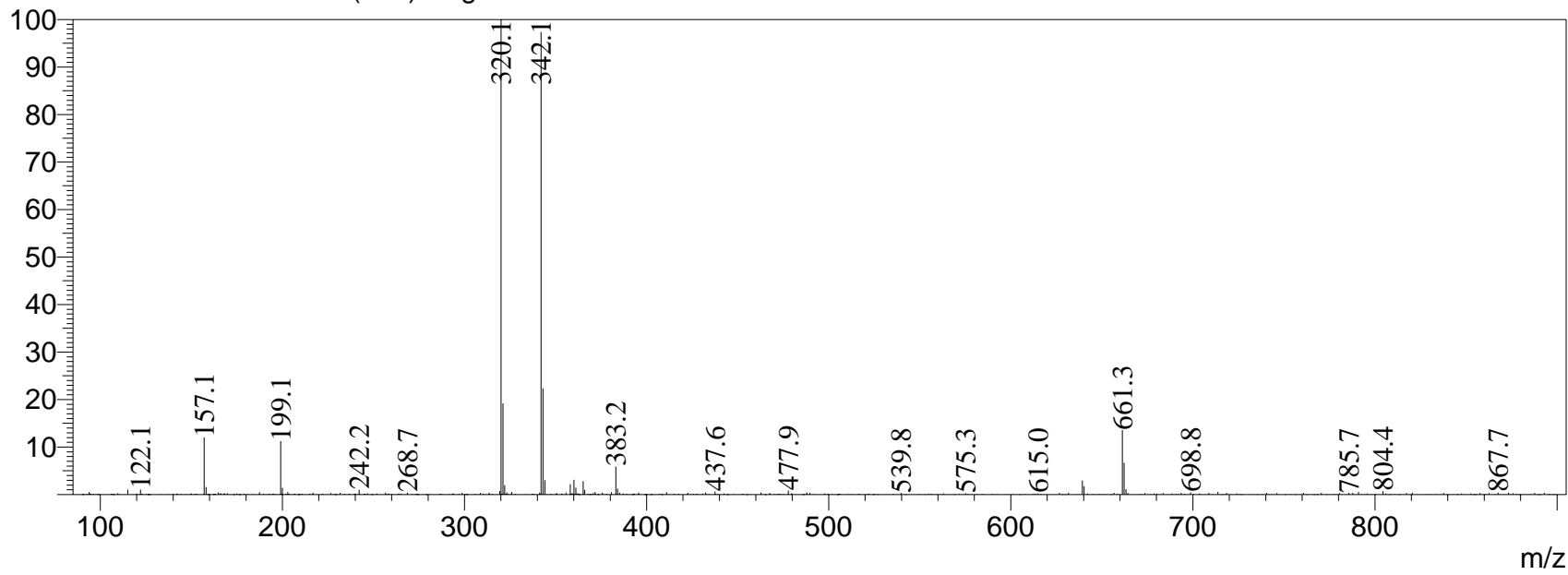




\section{LCMS Analysis Report}

\author{
Acquired by \\ Sample Name \\ Injection Volume \\ Data File \\ Method File \\ Report Format File \\ Date Acquired \\ Comment
}

: System Administrator

: LCMS43-PH-CYT-A-727-0-2(57211-032A1)1T

$: 2$

: LCMS43-PH-CYT-A-727-0-2(57211-032A1)1T.Icd

: ACN-Water-5mMNH4HCO3-10\%-50\%B-1.5-5.6MIN(90-900)+

: LCMS2020-PDA(DW)+ELSD+MS.Isr

: 2015-11-23 14:02:13

: Mobile phase A:Water/5mMNH4HCO3

Mobile phase B:Acetonitrile

Instrument Name:Shimadzu LCMS-2020

$<<$ Pump $>>$

Mode

Pump A

Pump B

Total Flow

B Conc.

: Binary gradient

: LC-30AD

: LC-30AD

$: 1.5000 \mathrm{~mL} / \mathrm{min}$

: $10.0 \%$

$<<$ Oven $>>$

Oven Temperature

: $40 \mathrm{C}$

$<<$ PDA $>>$

PDA Model

Lamp

Start Wavelength

End Wavelength

: SPD-M20A

: D2

: $190 \mathrm{~nm}$

: $400 \mathrm{~nm}$

$<<$ Interface $>>$

Interface

DL Temperature

Nebulizing Gas Flow

Heat Block

Drying Gas

<<MS Parameter>>

--Segment 1 Event 1--

Start Time

End Time

Acquisition Mode

Polarity

Event Time

Detector Voltage

Threshold

Start $\mathrm{m} / \mathrm{z}$

End $\mathrm{m} / \mathrm{z}$

Scan Speed

Interface Volt.

DL Volt.

Qarray DC Voltage

Qarray DC Voltage

System Configuration

$<<$ Column $>>$

Column Name

Length

Internal Diameter

Description

: Agilent Poroshell HPH-C1ऽ

: $50 \mathrm{~mm}$

$3.0 \mathrm{~mm}$

: $2.7 \mathrm{um}$
$<<$ LC Time Program $>>$

$\begin{array}{ll}\text { Time } & \text { Module } \\ 0.01 & \text { Pumps } \\ 3.00 & \text { Pumps } \\ 4.00 & \text { Pumps } \\ 5.00 & \text { Pumps } \\ 5.30 & \text { Pumps } \\ 5.60 & \text { Controller }\end{array}$

$\begin{array}{ll}\text { Command } & \text { Value } \\ \text { Pump B Conc. } & 10 \\ \text { Pump B Conc. } & 50 \\ \text { Pump B Conc. } & 95 \\ \text { Pump B Conc. } & 95 \\ \text { Pump B Conc. } & 10\end{array}$

Stop

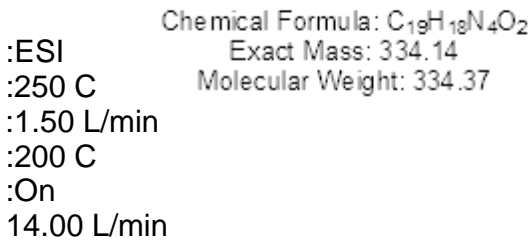

$: 0.00 \mathrm{~min}$

:5.00 min

:Scan

:Positive

$: 0.40 \mathrm{sec}$

$:+0.95 \mathrm{kV}$

$: 0$

$: 90.00$

:900.00

:2143 u/sec

:Use the Data in the Tuning File

:Use the Data in the Tuning File

:Use the Data in the Tuning File

:Use the Data in the Tuning File 
mAU
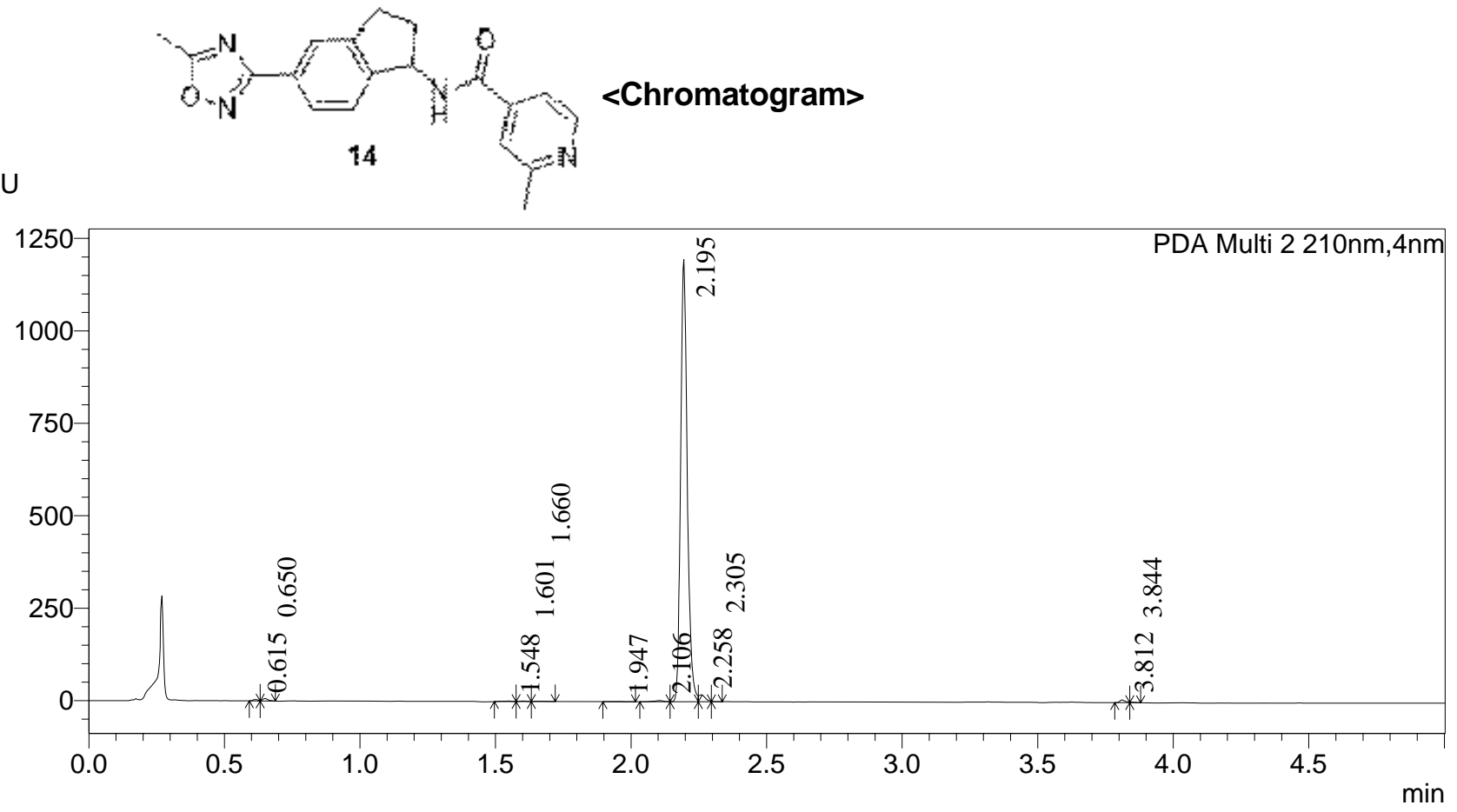

Peak Table

PDA Ch2 210nm

\begin{tabular}{|r|r|r|r|r|r|}
\hline Peak\# & Ret. Time & \multicolumn{1}{c|}{ Height } & Height\% & \multicolumn{1}{c|}{ Area } & \multicolumn{1}{c|}{ Area\% } \\
\hline 1 & 0.615 & 3762 & 0.322 & 4934 & 0.234 \\
\hline 2 & 0.650 & 5866 & 0.501 & 8553 & 0.405 \\
\hline 3 & 1.548 & 1265 & 0.108 & 2870 & 0.136 \\
\hline 4 & 1.601 & 1293 & 0.111 & 2068 & 0.098 \\
\hline 5 & 1.660 & 1362 & 0.116 & 2449 & 0.116 \\
\hline 6 & 1.947 & 967 & 0.083 & 2143 & 0.101 \\
\hline 7 & 2.106 & 3102 & 0.265 & 7714 & 0.365 \\
\hline 8 & 2.195 & 1125395 & 96.172 & 2038815 & 96.542 \\
\hline 9 & 2.258 & 17840 & 1.525 & 29425 & 1.393 \\
\hline 10 & 2.305 & 768 & 0.066 & 693 & 0.033 \\
\hline 11 & 3.812 & 6783 & 0.580 & 10262 & 0.486 \\
\hline 12 & 3.844 & 1782 & 0.152 & 1926 & 0.091 \\
\hline Total & & 1170185 & 100.000 & 2111853 & 100.000 \\
\hline
\end{tabular}

mAU

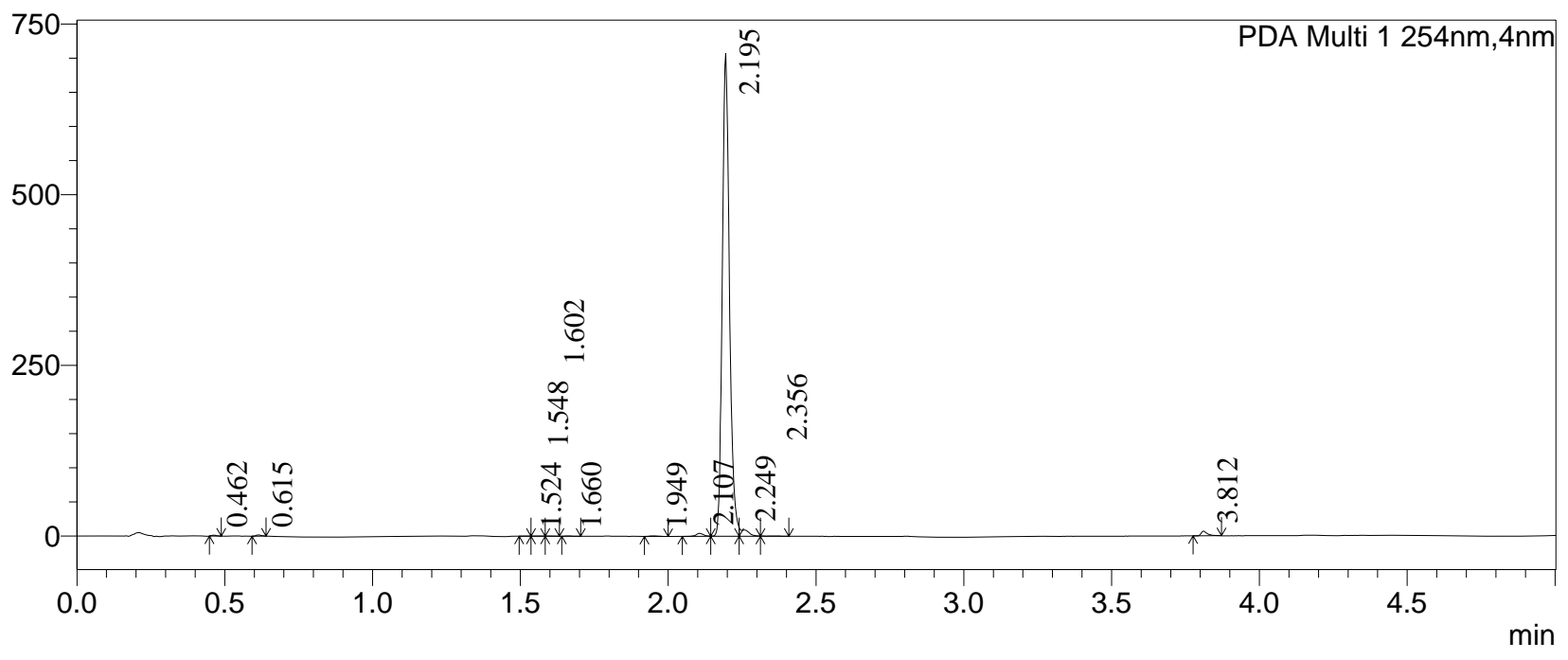

Peak Table

PDA Ch1 254nm

\begin{tabular}{|r|r|r|r|r|r|}
\hline Peak\# & Ret. Time & \multicolumn{1}{|c|}{ Height } & Height\% & Area & \multicolumn{1}{c|}{ Area\% } \\
\hline 1 & 0.462 & 1411 & 0.203 & 1542 & 0.125 \\
\hline 2 & 0.615 & 1750 & 0.252 & 2215 & 0.179 \\
\hline 3 & 1.524 & 567 & 0.082 & 770 & 0.062 \\
\hline 4 & 1.548 & 722 & 0.104 & 977 & 0.079 \\
\hline 5 & 1.602 & 725 & 0.105 & 1071 & 0.087 \\
\hline 6 & 1.660 & 513 & 0.074 & 886 & 0.072 \\
\hline 7 & 1.949 & 706 & 0.102 & 1258 & 0.102 \\
\hline 8 & 2.107 & 4253 & 0.613 & 7935 & 0.642 \\
\hline
\end{tabular}




\begin{tabular}{|r|r|r|r|r|r|}
\hline Peak\# & Ret. Time & \multicolumn{1}{c|}{ Height } & Height $\%$ & \multicolumn{1}{c|}{ Area } & \multicolumn{1}{c|}{ Area\% } \\
\hline 9 & 2.195 & 666824 & 96.156 & 1188603 & 96.177 \\
\hline 10 & 2.249 & 9408 & 1.357 & 19999 & 1.618 \\
\hline 11 & 2.356 & 213 & 0.031 & 669 & 0.054 \\
\hline 12 & 3.812 & 6389 & 0.921 & 9920 & 0.803 \\
\hline Total & & 693481 & 100.000 & 1235845 & 100.000 \\
\hline
\end{tabular}


$\mathrm{mV}$

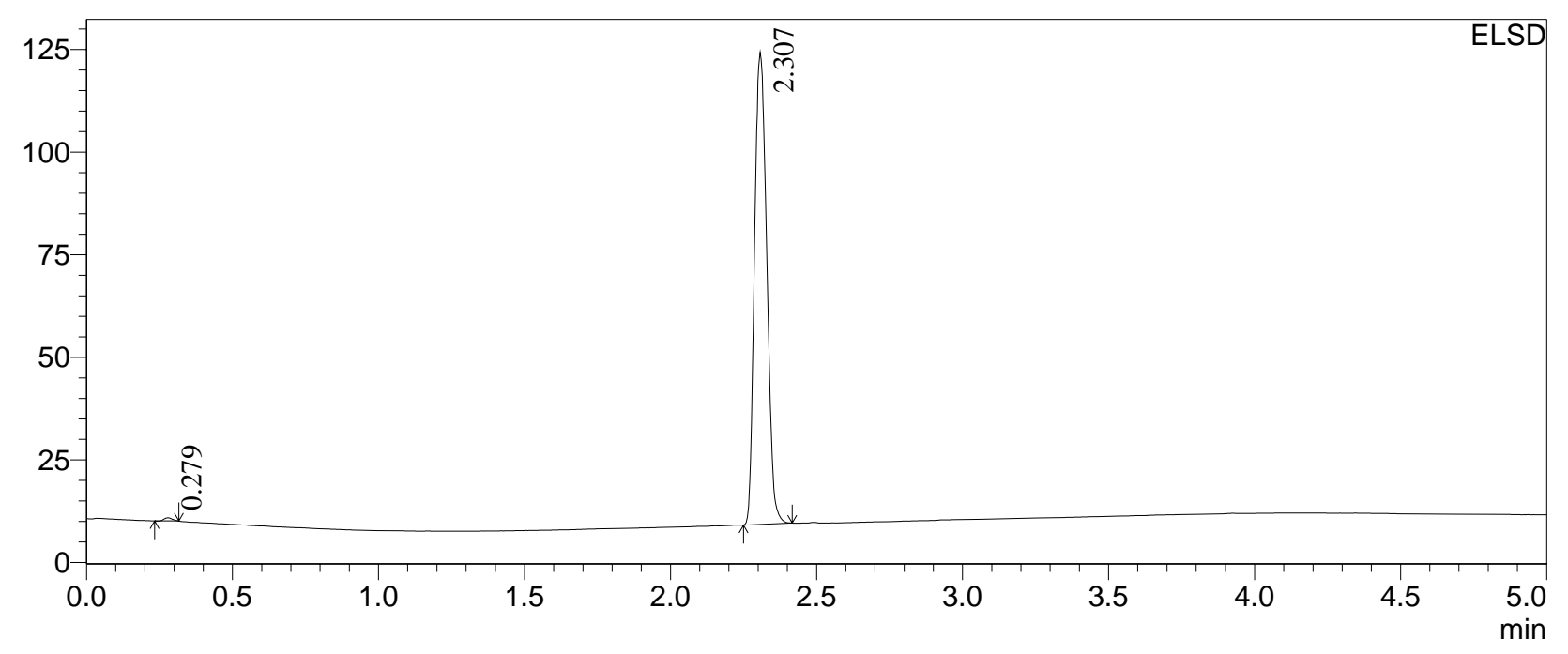

Peak Table

ELSD

\begin{tabular}{|r|r|r|r|r|r|}
\hline Peak\# & \multicolumn{1}{|c|}{ Ret. Time } & \multicolumn{1}{c|}{ Height } & Height\% & \multicolumn{1}{c|}{ Area } & \multicolumn{1}{c|}{ Area\% } \\
\hline 1 & 0.279 & 745 & 0.651 & 1426 & 0.425 \\
\hline 2 & 2.307 & 113790 & 99.349 & 334492 & 99.575 \\
\hline Total & & 114536 & 100.000 & 335918 & 100.000 \\
\hline
\end{tabular}

Line\#:1 R.Time:2.193(Scan\#:337)

Mass Spectrum

MassPeaks:336

Spectrum Mode:Averaged 2.186-2.200(336-338) BasePeak:335.1(3520623)

BG Mode:Calc Segment 1 - Event 1

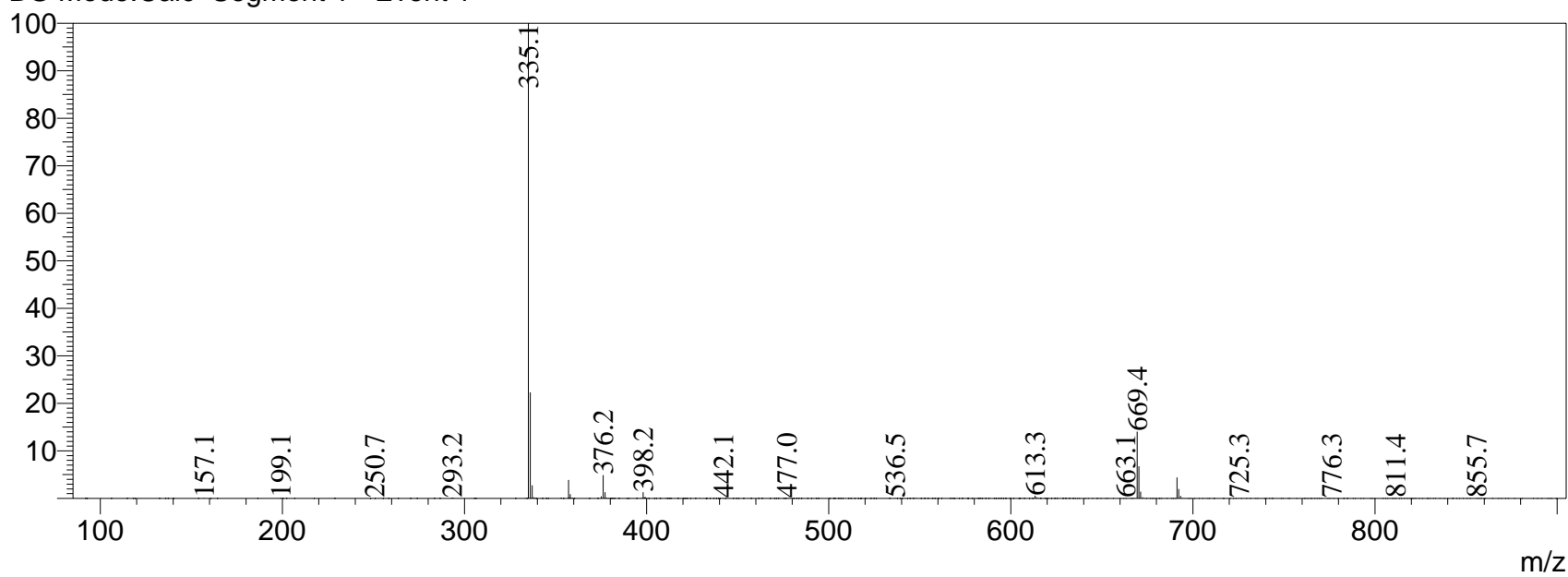




\section{LCMS Analysis Report}

\section{Acquired by \\ Sample Name Injection Volume \\ Data File \\ Method File \\ Report Format File \\ Date Acquired \\ Comment}

: System Administrator

: LCMS52-PH-CYT-A-862-0-2(57137-145A1)1T

$: 1$

: LCMS52-PH-CYT-A-862-0-2(57137-145A1)1T.Icd

: ACN-Water-H2O-10\%B-1.3-3.0min(90-900).Icm

: LCMS2020-PDA+ELSD+TIC+MS.Isr

: 1/14/2016 17:36:48

: Mobile phaseA:water/5mM NH4HCO3;

Mobile phaseB:Acetonitrile
Instrument Name:Shimadzu LCMS-2020

$<<$ Pump $>>$

Mode

Pump A

Pump B

Total Flow

B Conc.

$<<$ Oven $>>$

Oven Temperature

$<<$ PDA $>$

PDA Model

Lamp

Start Wavelength

End Wavelength
: Binary gradient

: LC-20ADXR

: LC-20ADXR

: $1.3000 \mathrm{~mL} / \mathrm{min}$

: $10.0 \%$

$: 40 \mathrm{C}$

SPD-M20A

: D2

: $190 \mathrm{~nm}$

: $400 \mathrm{~nm}$

<<Interface>>
Interface
DL Temperature
Nebulizing Gas Flow
Heat Block
Drying Gas
<<MS Parameter>>
--Segment 1 Event 1--
Start Time
End Time
Acquisition Mode
Polarity
Event Time
Detector Voltage
Threshold
Start m/z
End m/z
Scan Speed
Interface Volt.
DL Volt.
Qarray DC Voltage
Qarray DC Voltage

:ESI

:250 C

:1.50 L/min

:250 C

:On

$15.00 \mathrm{~L} / \mathrm{min}$

$: 0.00 \mathrm{~min}$

:3.00 min

:Scan

:Positive

$: 0.60 \mathrm{sec}$

:+0.90 kV

$: 0$

$: 90.00$

:900.00

:1364 u/sec

:Use the Data in the Tuning File

:Use the Data in the Tuning File

:Use the Data in the Tuning File

:Use the Data in the Tuning File
System Configuration

$<<$ Column $>>$

Column Name

Length

Internal Diameter

Description

$<<$ LC Time Program >>
Time
0.01
2.10
2.70
2.75
3.00

: Kinetex EVO C18 100A

: $50 \mathrm{~mm}$

: $3.0 \mathrm{~mm}$

: $2.6 \mathrm{um}$

$\begin{array}{lll}\text { Module } & \text { Command } & \text { Value } \\ \text { Pumps } & \text { Pump B Conc. } & 10 \\ \text { Pumps } & \text { Pump B Conc. } & 95 \\ \text { Pumps } & \text { Pump B Conc. } & 95 \\ \text { Pumps } & \text { Pump B Conc. } & 10 \\ \text { Controller } & \text { Stop } & \end{array}$


mAU
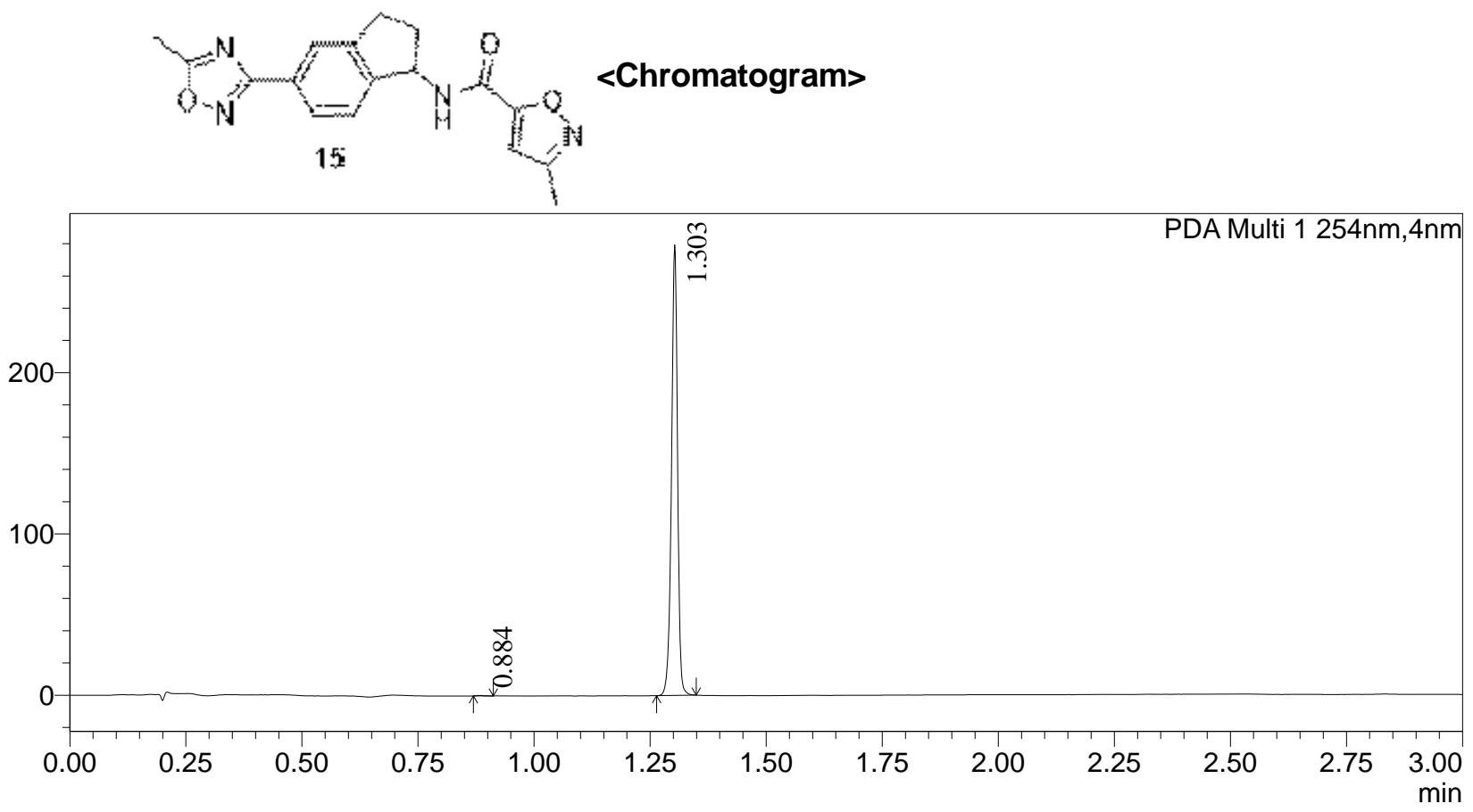

Peak Table

PDA Ch1 254nm
\begin{tabular}{|r|r|r|r|r|r|}
\hline Peak\# & Ret. Time & \multicolumn{1}{|c|}{ Height } & Height $\%$ & \multicolumn{1}{|c|}{ Area } & \multicolumn{1}{|c|}{ Area $\%$} \\
\hline 1 & 0.884 & 332 & 0.120 & 305 & 0.120 \\
\hline 2 & 1.303 & 277096 & 99.880 & 253506 & 99.880 \\
\hline Total & & 277428 & 100.000 & 253811 & 100.000 \\
\hline
\end{tabular}

mAU

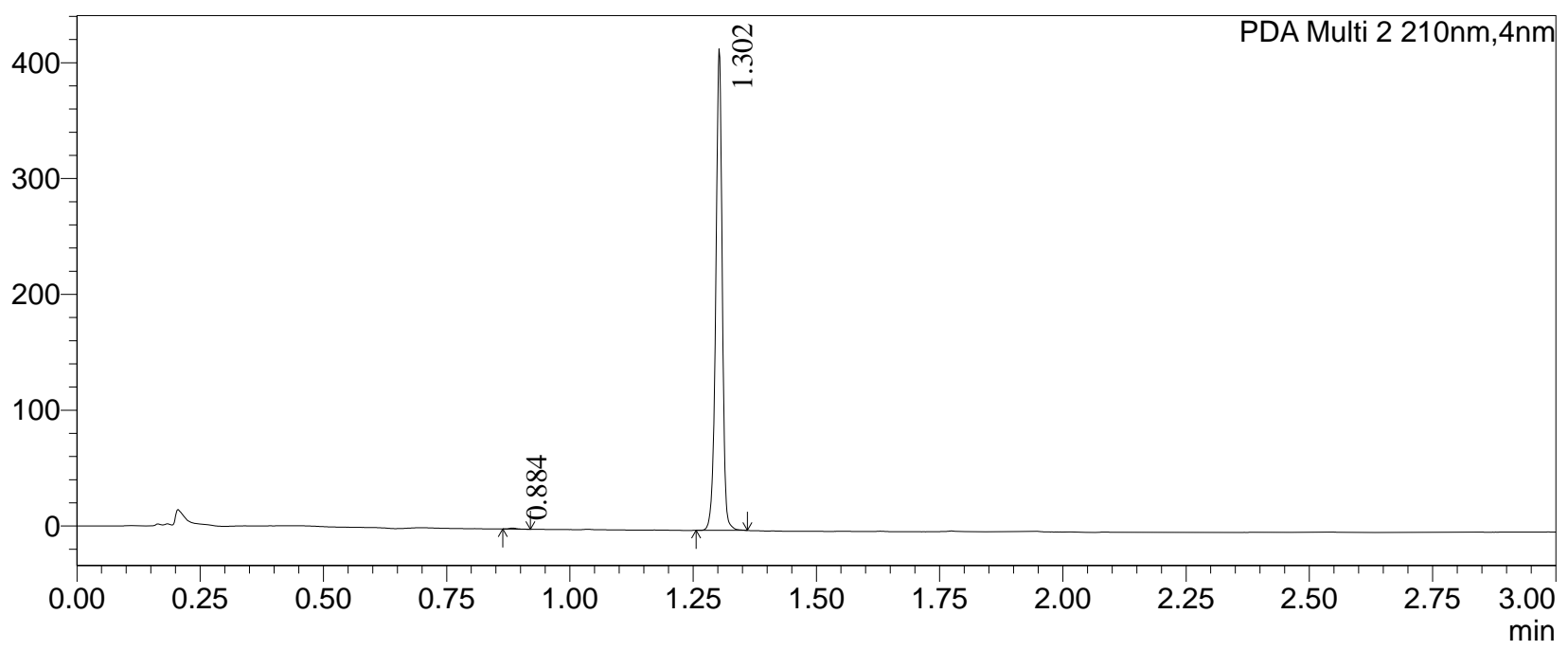

Peak Table

PDA Ch2 210nm

\begin{tabular}{|r|r|r|r|r|r|}
\hline Peak\# & \multicolumn{1}{|c|}{ Ret. Time } & \multicolumn{1}{c|}{ Height } & \multicolumn{1}{c|}{ Height\% } & \multicolumn{1}{c|}{ Area } & \multicolumn{1}{c|}{ Area\% } \\
\hline 1 & 0.884 & 796 & 0.220 & 826 & 0.218 \\
\hline 2 & 1.302 & 361127 & 99.780 & 378710 & 99.782 \\
\hline Total & & 361923 & 100.000 & 379536 & 100.000 \\
\hline
\end{tabular}


$\mathrm{mV}$

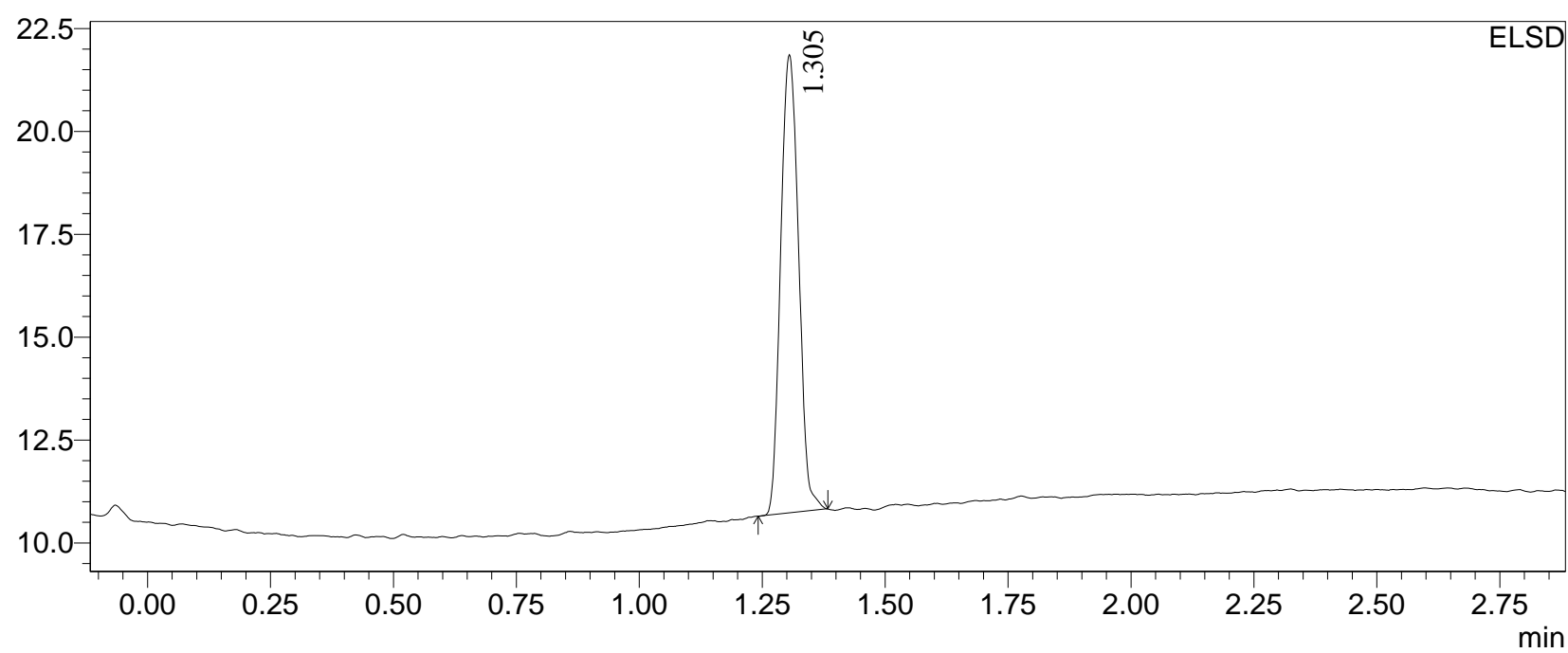

Peak Table

ELSD

\begin{tabular}{|r|r|r|r|r|r|}
\hline Peak\# & Ret. Time & \multicolumn{1}{|c|}{ Height } & \multicolumn{1}{c|}{ Height\% } & \multicolumn{1}{c|}{ Area } & \multicolumn{1}{c|}{ Area $\%$} \\
\hline 1 & 1.305 & 11040 & 100.000 & 28234 & 100.000 \\
\hline Total & & 11040 & 100.000 & 28234 & 100.000 \\
\hline
\end{tabular}

Line\#:1 R.Time:1.300(Scan\#:135)

Mass Spectrum

MassPeaks:367

Spectrum Mode:Single 1.300(135) BasePeak:325.1(23570)

BG Mode:Averaged 1.280-1.350(133-140) Segment 1 - Event 1

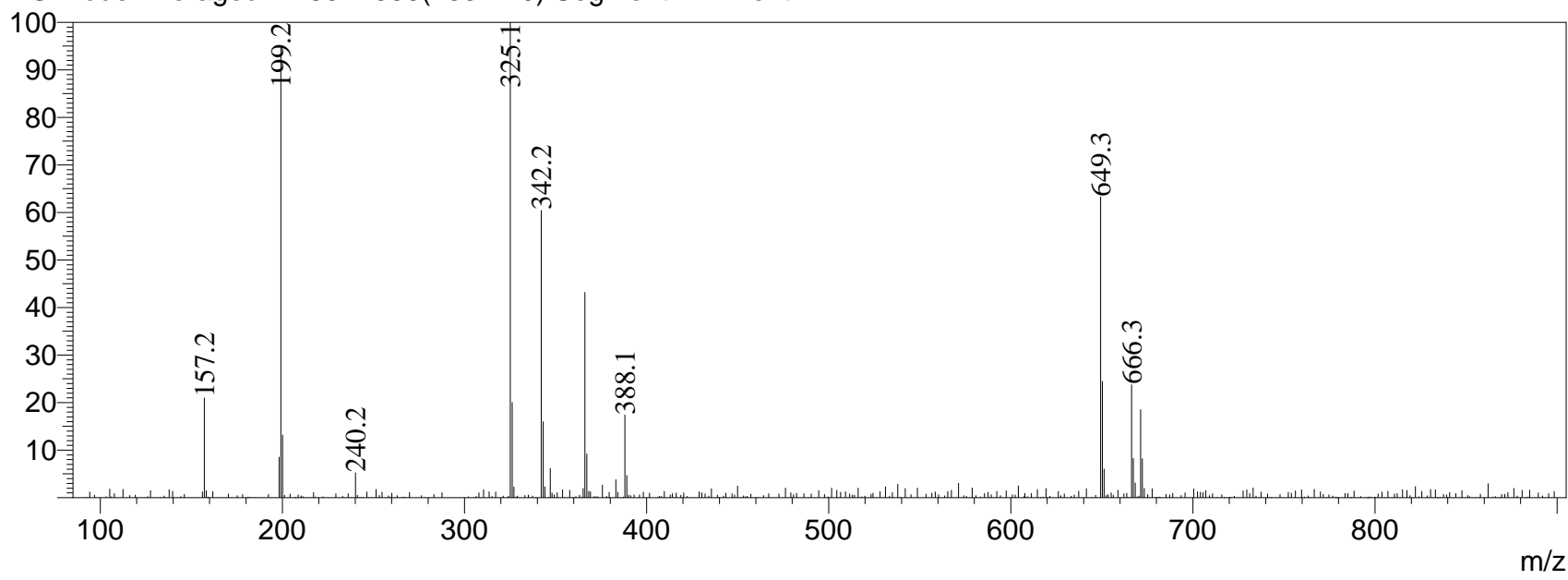




\section{LCMS Analysis Report}

\author{
Acquired by \\ Sample Name \\ Injection Volume \\ Data File \\ Method File \\ Report Format File \\ Date Acquired \\ Comment
}

: Admin

: LCMS41-PH-CYT-A-882-0-2(57137-151A1)2T

$: 3$

: LCMS41-PH-CYT-A-882-0-2(57137-151A1)2T.Icd

: ACN-Water-5\%B-1.5-3.0MIN(90-900).Icm

: DefaultLCMS.Icr

: 2016-1-18 13:11:36

: Mobile phase A: Water/0.05\%TFA

Mobile phase B: Acetonitrile/0.05\%TFA
Instrument Name:Shimadzu LCMS-2020

$<<$ Pump $>>$

Mode

Pump A

Pump B

Total Flow

B Conc.

$<<$ Oven $>>$

Oven Temperature

$<<$ PDA $>$

PDA Model

Lamp

Start Wavelength

End Wavelength

System Configuration

$<<$ Column $>>$

Column Name

Length

Internal Diameter
: Binary gradient

: LC-20AD

: LC-20AD

: $1.5000 \mathrm{~mL} / \mathrm{min}$

: $5.0 \%$

$: 40 \mathrm{C}$

: SPD-M20A

: D2

: $190 \mathrm{~nm}$

: $800 \mathrm{~nm}$

<<Interface>>
Interface
DL Temperature
Nebulizing Gas Flow
Heat Block
<<MS Parameter>>
--Segment 1 Event 1--
Start Time
End Time
Acquisition Mode
Polarity
Event Time
Detector Voltage
Threshold
Start m/z
End m/z
Scan Speed
Interface Voltage
DL Volt.
Qarray Voltage

: Ascentis Express C18 2.7um

: $50 \mathrm{~mm}$

: $3.0 \mathrm{~mm}$

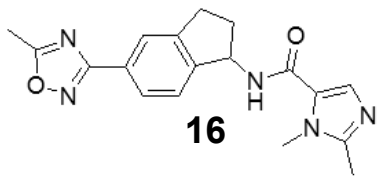

Chemical Formula: $\mathrm{C}_{18} \mathrm{H}_{19} \mathrm{~N}_{5} \mathrm{O}_{2}$ Exact Mass: 337.1539 Molecular Weight: 337.3758

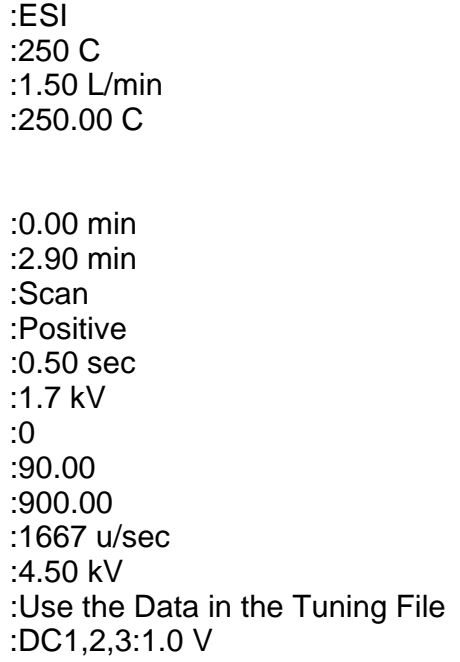

$\begin{array}{llll}<<\text { LC Time Program }>> & & & \text { Value } \\ \text { Time } & \text { Module } & \text { Command } & 5 \\ 0.01 & \text { Pumps } & \text { Pump B Conc. } & 100 \\ 2.00 & \text { Pumps } & \text { Pump B Conc. } & 100 \\ 2.60 & \text { Pumps } & \text { Pump B Conc. } & 5 \\ 2.70 & \text { Pumps } & \text { Pump B Conc. } & \\ 3.00 & \text { Controller } & \text { Stop } & \end{array}$




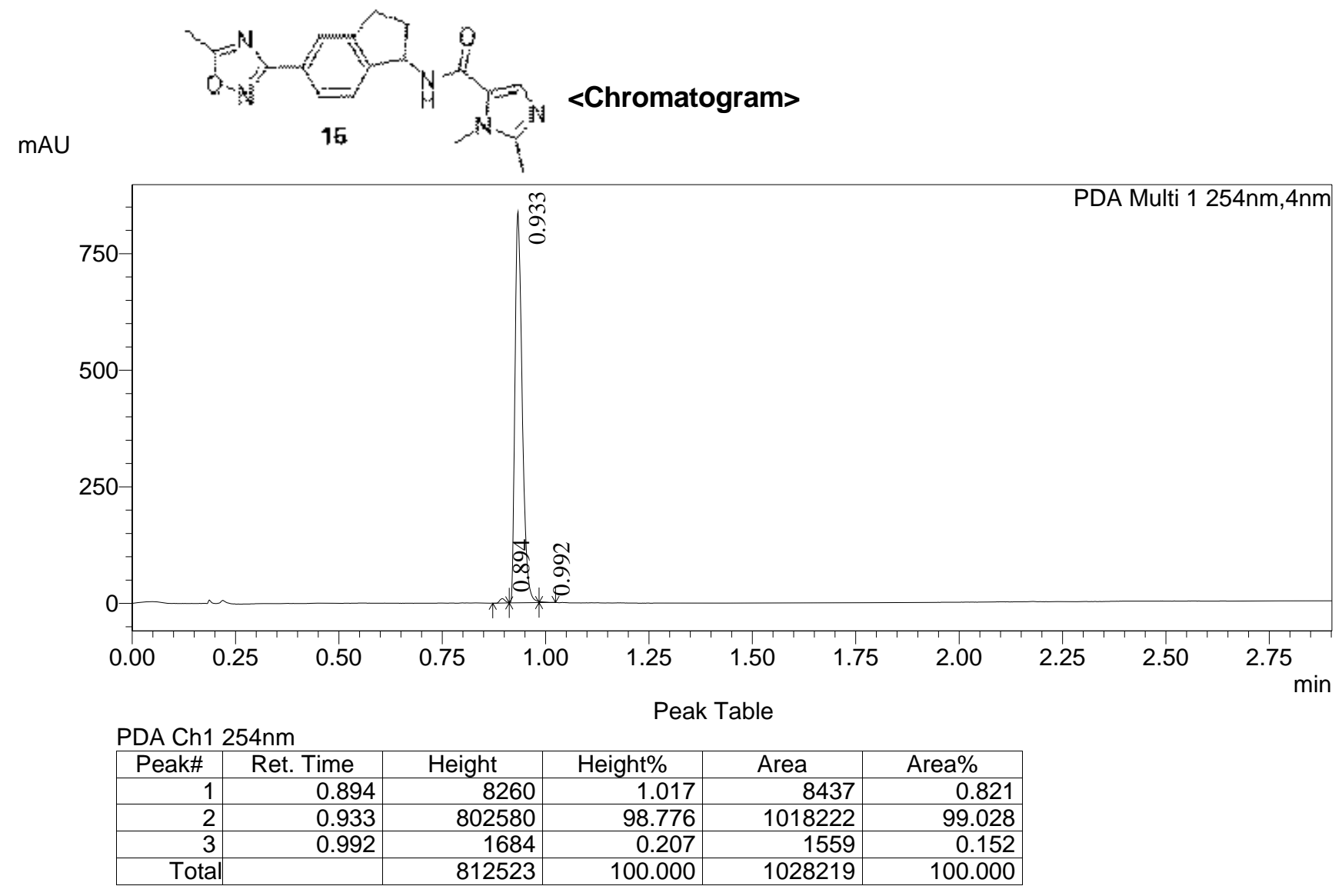

mAU

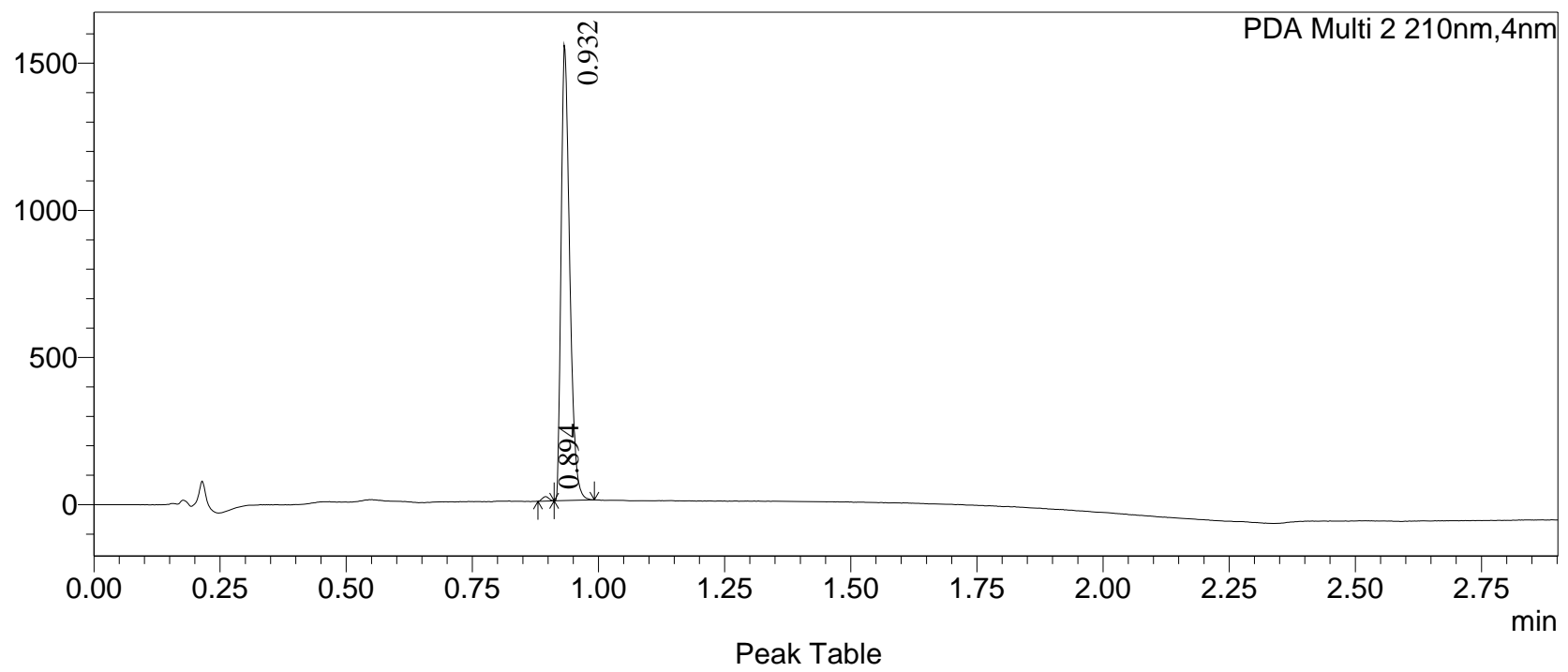

PDA Ch2 210nm

\begin{tabular}{|r|r|r|r|r|r|}
\hline Peak\# & \multicolumn{1}{|c|}{ Ret. Time } & \multicolumn{1}{|c|}{ Height } & Height\% & \multicolumn{1}{c|}{ Area } & \multicolumn{1}{c|}{ Area\% } \\
\hline 1 & 0.894 & 15129 & 1.017 & 13647 & 0.737 \\
\hline 2 & 0.932 & 1472352 & 98.983 & 1836965 & 99.263 \\
\hline Total & & 1487481 & 100.000 & 1850612 & 100.000 \\
\hline
\end{tabular}


$\mathrm{mV}$

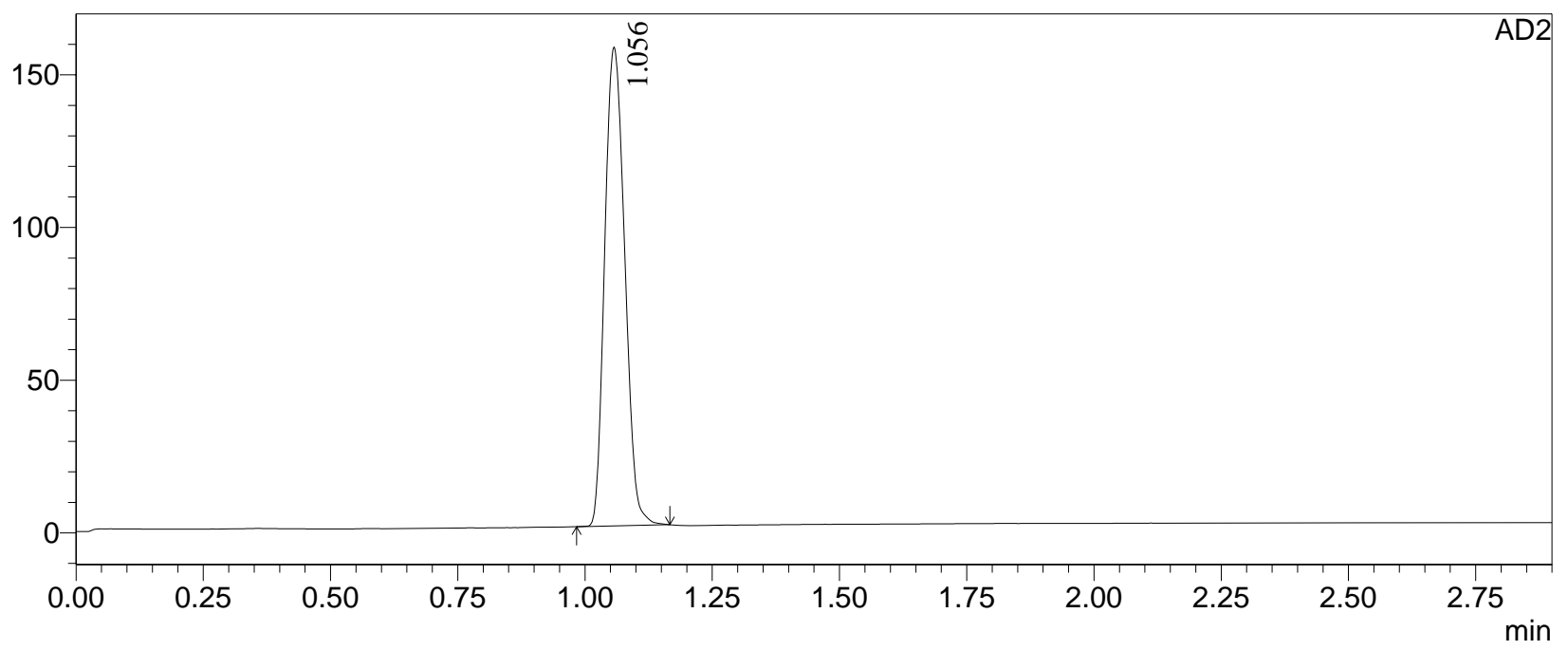

Peak Table

AD2

\begin{tabular}{|r|r|r|r|r|r|}
\hline Peak\# & Ret. Time & Height & Height\% & \multicolumn{1}{c|}{ Area } & \multicolumn{1}{c|}{ Area\% } \\
\hline 1 & 1.056 & 154776 & 100.000 & 429007 & 100.000 \\
\hline Total & & 154776 & 100.000 & 429007 & 100.000 \\
\hline
\end{tabular}

Line\#:1 R.Time:0.933(Scan\#:117)

Mass Spectrum

MassPeaks: 412

Spectrum Mode:Single 0.933(117) BasePeak:338.1(441279)

BG Mode:Averaged 0.858-1.050(108-131) Segment 1 - Event 1

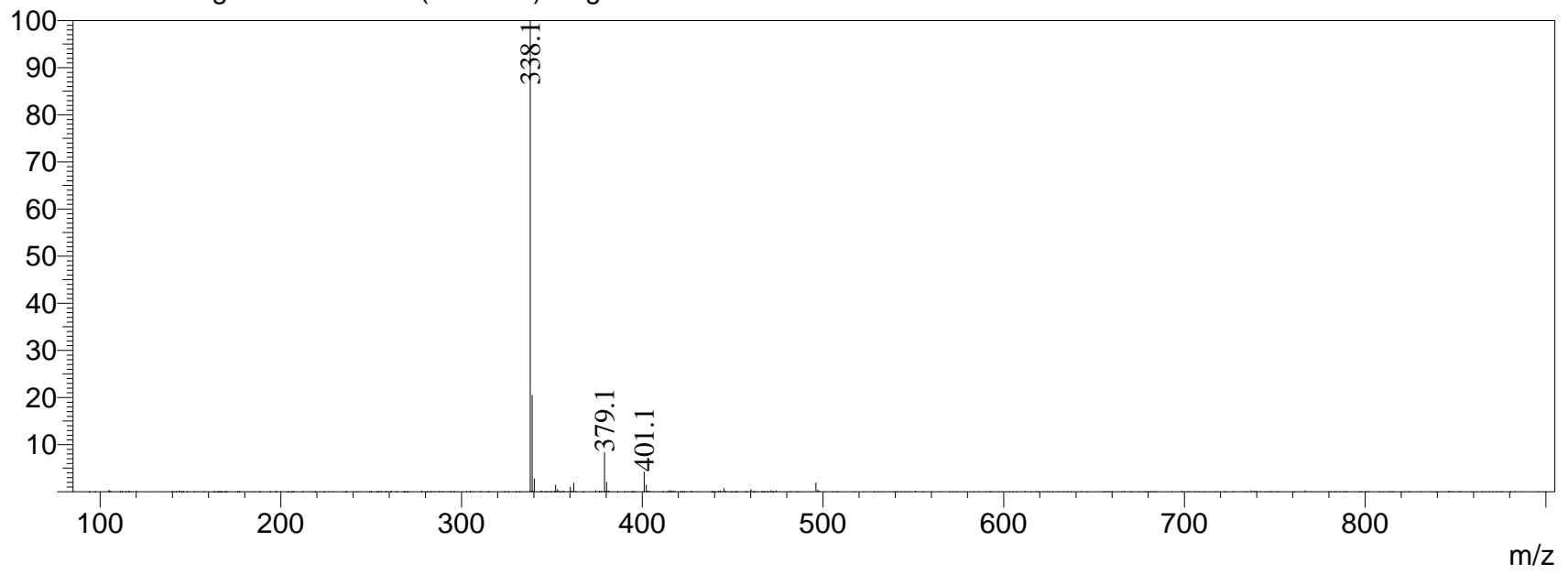


Acquired by

Sample Name

Sample ID

Injection Volume

Data File Name

Method File Name

Report File Name

Data Acquired

Data Processed

Column

Size

Mobile phase

Pressure

Flow

Instrument

Detector

Temperature
Chiral HPLC

: System Administrator

: CHIRAL_HPLC-PH-CYT-A-904-0-1(56385-135RS)1T

: 5 uL

: PDA1601168.Icd

: Chiral.Icm

: DEFAULT.Isr

: 2016-1-25 16:29:59

: 2016-1-25 17:00:19

:Chiral Cellulose-SB

$: 0.46 * 15 \mathrm{~cm} ; 3 \mathrm{um}$

$: \mathrm{Hex}(0.1 \% \mathrm{DEA}): \mathrm{EtOH}=50: 50$

$: \mathrm{MPa}$

$: 1.0 \mathrm{ml} / \mathrm{min}$

:LC-37

:UV-254nm

$: 25^{\circ} \mathrm{C}$ and

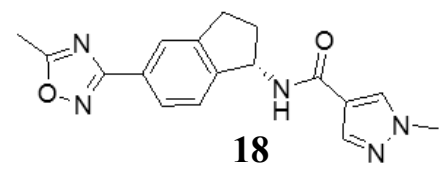

Chromatogram

CHIRAL_HPLC-PH-CYT-A-904-0-1(56385-135RS)1T D:ILC37datal2016।201601|PDA1601168.lcd

uAU

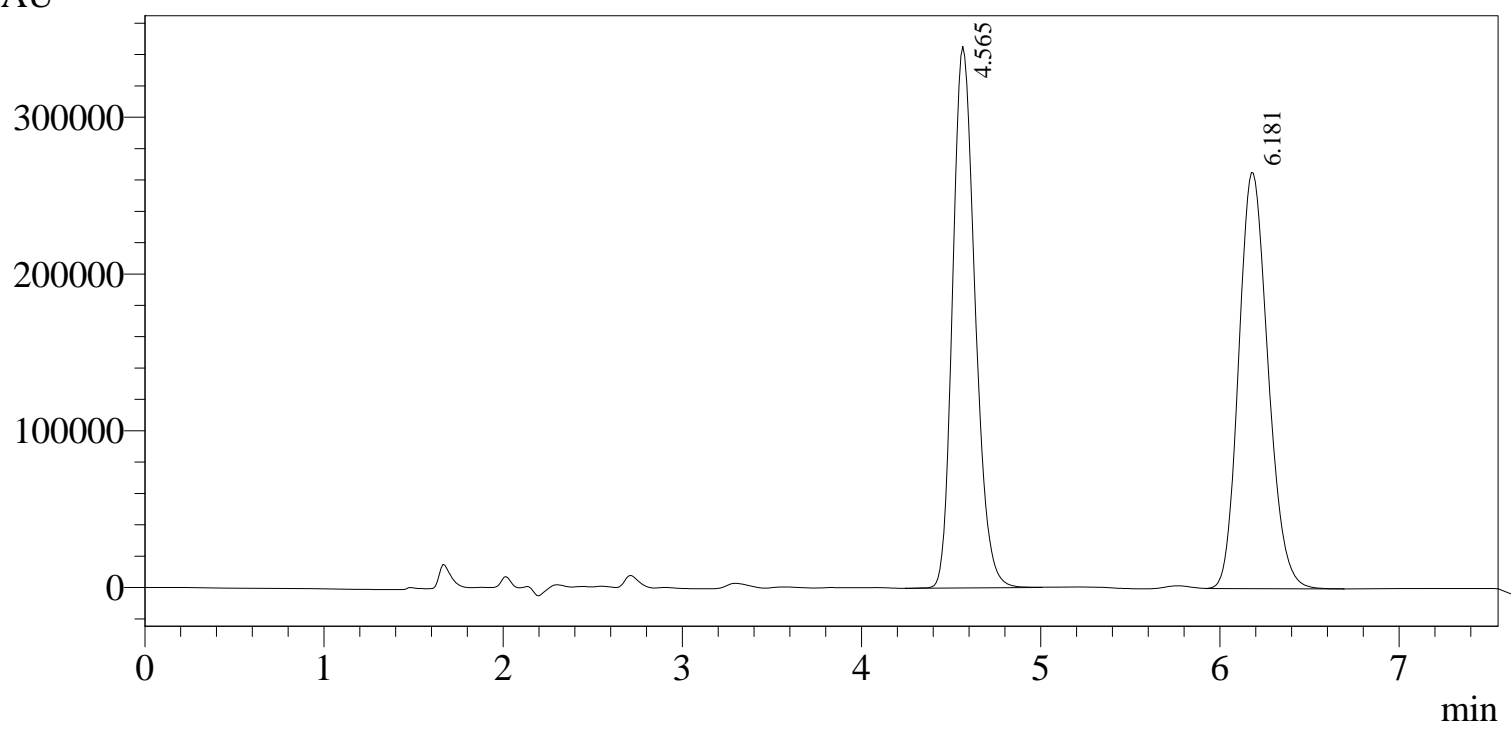

1PDA Multi 1

1 PDA Multi $1 / 220 \mathrm{~nm}, 4 \mathrm{~nm}$

PeakTable

PDA Ch1 220nm

\begin{tabular}{|r|r|r|r|r|r|}
\hline \multicolumn{1}{|c|}{ Peak\# } & Ret. Time & Height & \multicolumn{1}{c|}{ Area } & \multicolumn{1}{c|}{ Height \% } & \multicolumn{1}{c|}{ Area \% } \\
\hline 1 & 4.565 & 345545 & 3012058 & 56.554 & 49.950 \\
\hline 2 & 6.181 & 265452 & 3018099 & 43.446 & 50.050 \\
\hline Total & & 610997 & 6030157 & 100.000 & 100.000 \\
\hline
\end{tabular}


Acquired by

Sample Name

Sample ID

Injection Volume

Data File Name

Method File Name

Report File Name

Data Acquired

Data Processed

Column

Size

Mobile phase

Pressure

Flow

Instrument

Detector

Temperature
Chiral HPLC

: System Administrator

: CHIRAL_HPLC-PH-CYT-A-904-0-1(56385-135H1)1T

: 5 uL

: PDA1601169.Icd

: Chiral.Icm

: DEFAULT.Isr

: 2016-1-25 16:38:28

: 2016-1-25 16:59:49

:Chiral Cellulose-SB

$: 0.46$ * $15 \mathrm{~cm} ; 3 \mathrm{um}$

$: \operatorname{Hex}(0.1 \% \mathrm{DEA}): \mathrm{EtOH}=50: 50$

$: \mathrm{MPa}$

$: 1.0 \mathrm{ml} / \mathrm{min}$

:LC-37

:UV-254nm

$: 25^{\circ} \mathrm{C}$

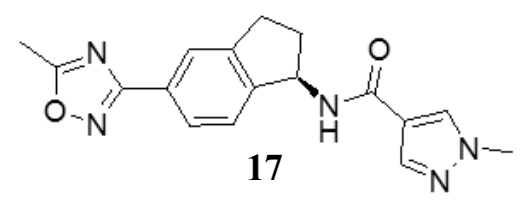

Chromatogram

CHIRAL_HPLC-PH-CYT-A-904-0-1(56385-135H1)1T D:ILC37data\2016।201601\PDA1601169.lcd

uAU

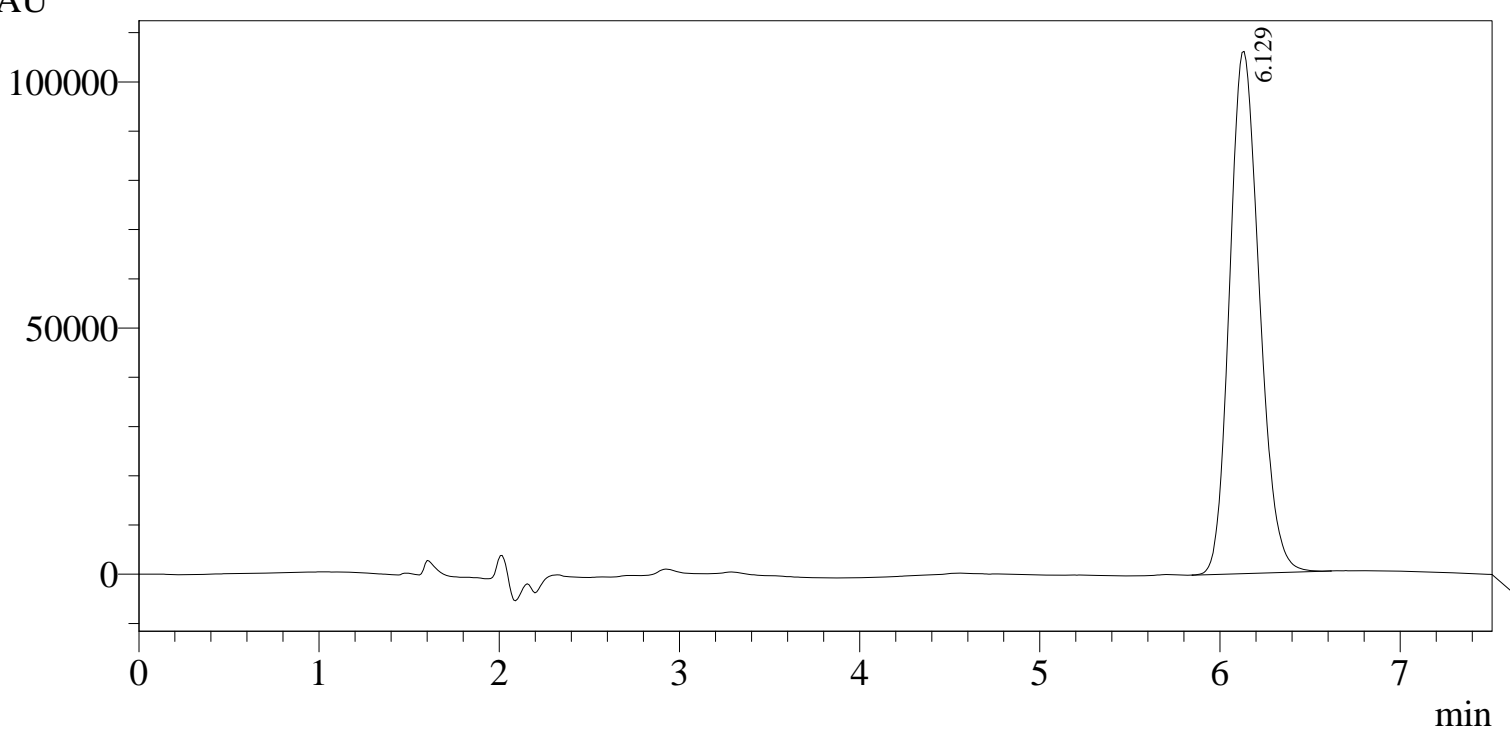

1 PDA Multi 1 / 220nm,4nm

PeakTable

PDA Ch1 220nm

\begin{tabular}{|r|r|r|r|r|r|}
\hline \multicolumn{1}{|c|}{ Peak\# } & Ret. Time & Height & \multicolumn{1}{c|}{ Area } & Height \% & \multicolumn{1}{c|}{ Area \% } \\
\hline 1 & 6.129 & 106082 & 1220887 & 100.000 & 100.000 \\
\hline Total & & 106082 & 1220887 & 100.000 & 100.000 \\
\hline
\end{tabular}




\section{LCMS Analysis Report}

\section{Acquired by \\ Sample Name Injection Volume \\ Data File \\ Method File \\ Report Format File \\ Date Acquired \\ Comment}

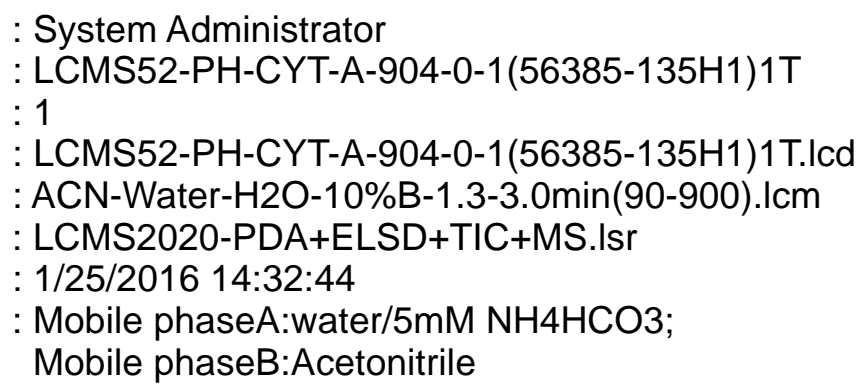

: System Administrator

: LCMS52-PH-CYT-A-904-0-1(56385-135H1)1T

$: 1$

: LCMS52-PH-CYT-A-904-0-1(56385-135H1)1T.Icd

: ACN-Water-H2O-10\%B-1.3-3.0min(90-900).Icm

: LCMS2020-PDA+ELSD+TIC+MS.Isr

: 1/25/2016 14:32:44

: Mobile phaseA:water/5mM NH4HCO3;

Mobile phaseB:Acetonitrile

Instrument Name:Shimadzu LCMS-2020

$<<$ Pump $>>$

Mode

Pump A

Pump B

Total Flow

B Conc.

: Binary gradient

: LC-20ADXR

: LC-20ADXR

: $1.3000 \mathrm{~mL} / \mathrm{min}$

: $10.0 \%$

$<<$ Oven $>>$

Oven Temperature

$: 40 \mathrm{C}$

$<<$ PDA $>>$

PDA Model

Lamp

SPD-M20A

: D2

: $190 \mathrm{~nm}$

Start Wavelength

End Wavelength

: $400 \mathrm{~nm}$

<<Interface>>
Interface
DL Temperature
Nebulizing Gas Flow
Heat Block
Drying Gas
<<MS Parameter>>
--Segment 1 Event 1--
Start Time
End Time
Acquisition Mode
Polarity
Event Time
Detector Voltage
Threshold
Start m/z
End m/z
Scan Speed
Interface Volt.
DL Volt.
Qarray DC Voltage
Qarray DC Voltage

:ESI

:250 C

:1.50 L/min

:250 C

:On

$15.00 \mathrm{~L} / \mathrm{min}$

$: 0.00 \mathrm{~min}$

:3.00 min

:Scan

:Positive

$: 0.60 \mathrm{sec}$

:+0.90 kV

$: 0$

$: 90.00$

:900.00

:1364 u/sec

:Use the Data in the Tuning File

:Use the Data in the Tuning File

:Use the Data in the Tuning File

:Use the Data in the Tuning File
System Configuration

$<<$ Column $>>$

Column Name

Length

Internal Diameter

Description

<LC Time Program>>
Time
0.01
2.10
2.70
2.75
3.00

: Kinetex EVO C18 100A

: $50 \mathrm{~mm}$

: $3.0 \mathrm{~mm}$

: $2.6 \mathrm{um}$

$\begin{array}{lll}\text { Module } & \text { Command } & \text { Value } \\ \text { Pumps } & \text { Pump B Conc. } & 10 \\ \text { Pumps } & \text { Pump B Conc. } & 95 \\ \text { Pumps } & \text { Pump B Conc. } & 95 \\ \text { Pumps } & \text { Pump B Conc. } & 10 \\ \text { Controller } & \text { Stop } & \end{array}$



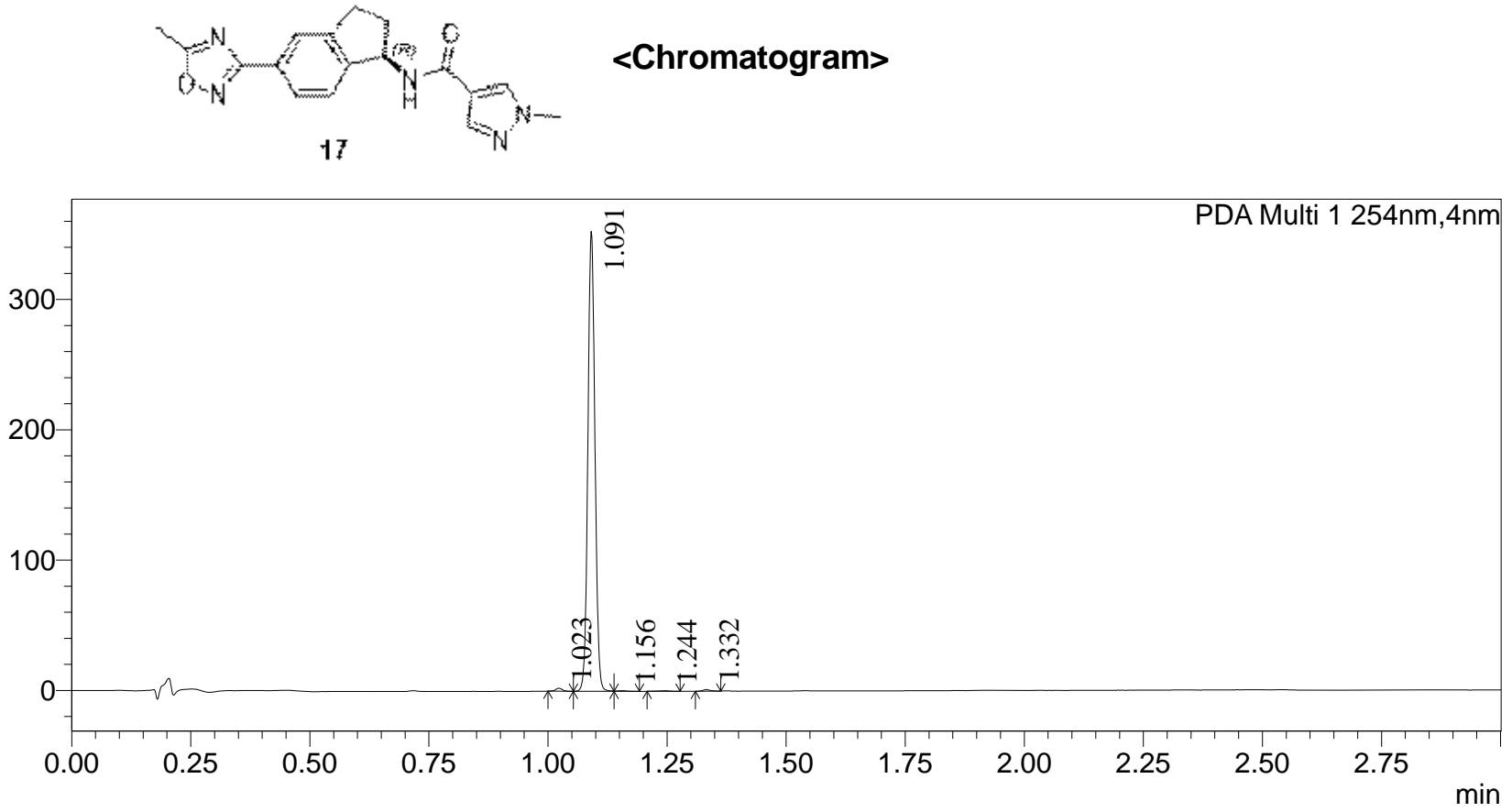

Peak Table

PDA Ch1 254nm

\begin{tabular}{|r|r|r|r|r|r|}
\hline Peak\# & Ret. Time & \multicolumn{1}{|c|}{ Height } & Height\% & \multicolumn{1}{|c|}{ Area } & \multicolumn{1}{c|}{ Area\% } \\
\hline 1 & 1.023 & 2240 & 0.635 & 2224 & 0.603 \\
\hline 2 & 1.091 & 348643 & 98.762 & 364155 & 98.735 \\
\hline 3 & 1.156 & 454 & 0.129 & 620 & 0.168 \\
\hline 4 & 1.244 & 438 & 0.124 & 541 & 0.147 \\
\hline 5 & 1.332 & 1236 & 0.350 & 1280 & 0.347 \\
\hline Total & & 353013 & 100.000 & 368820 & 100.000 \\
\hline
\end{tabular}

mAU

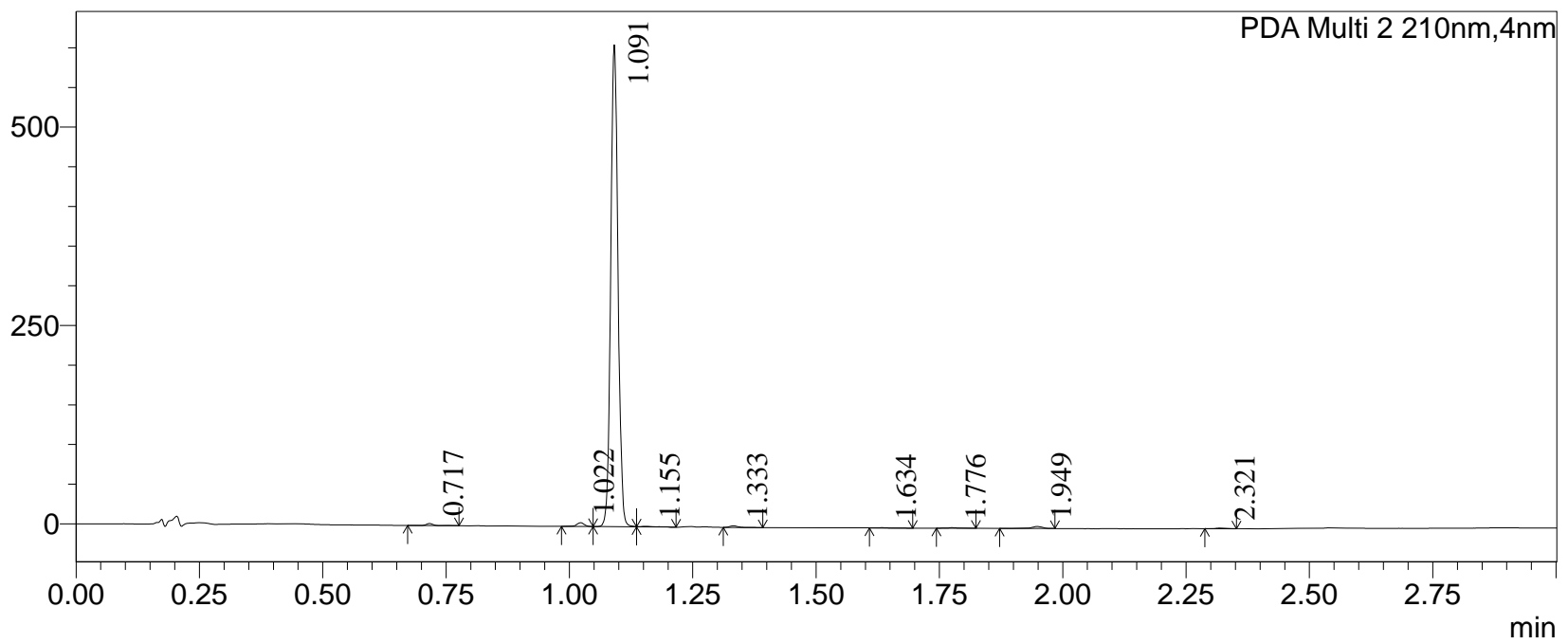

Peak Table

PDA Ch2 210nm

\begin{tabular}{|r|r|r|r|r|r|}
\hline Peak\# & Ret. Time & \multicolumn{1}{c|}{ Height } & Height $\%$ & \multicolumn{1}{|c|}{ Area } & \multicolumn{1}{c|}{ Area\% } \\
\hline 1 & 0.717 & 2385 & 0.418 & 2221 & 0.345 \\
\hline 2 & 1.022 & 4397 & 0.770 & 5279 & 0.820 \\
\hline 3 & 1.091 & 557538 & 97.619 & 626135 & 97.290 \\
\hline 4 & 1.155 & 692 & 0.121 & 1201 & 0.187 \\
\hline 5 & 1.333 & 1926 & 0.337 & 2340 & 0.364 \\
\hline 6 & 1.634 & 367 & 0.064 & 562 & 0.087 \\
\hline 7 & 1.776 & 567 & 0.099 & 955 & 0.148 \\
\hline 8 & 1.949 & 2449 & 0.429 & 3821 & 0.594 \\
\hline 9 & 2.321 & 815 & 0.143 & 1066 & 0.166 \\
\hline Total & & 571136 & 100.000 & 643579 & 100.000 \\
\hline
\end{tabular}


$\mathrm{mV}$

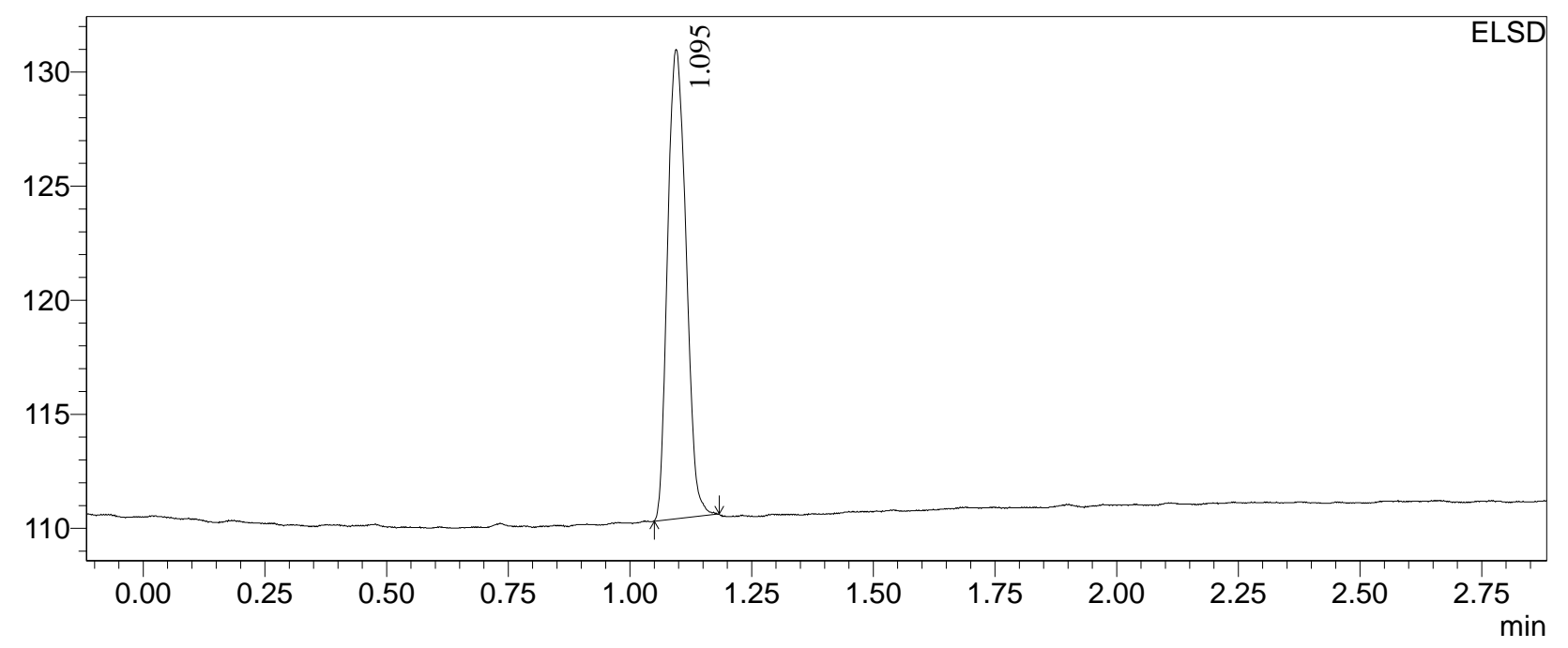

Peak Table

ELSD

\begin{tabular}{|r|r|r|r|r|r|}
\hline Peak\# & Ret. Time & Height & \multicolumn{1}{c|}{ Height\% } & \multicolumn{1}{c|}{ Area } & \multicolumn{1}{c|}{ Area\% } \\
\hline 1 & 1.095 & 20357 & 100.000 & 54579 & 100.000 \\
\hline Total & & 20357 & 100.000 & 54579 & 100.000 \\
\hline
\end{tabular}

Line\#:1 R.Time:1.090(Scan\#:114)

Mass Spectrum

MassPeaks:351

Spectrum Mode:Single 1.090(114) BasePeak:324.1(257182)

BG Mode:Averaged 1.050-1.150(110-120) Segment 1 - Event 1

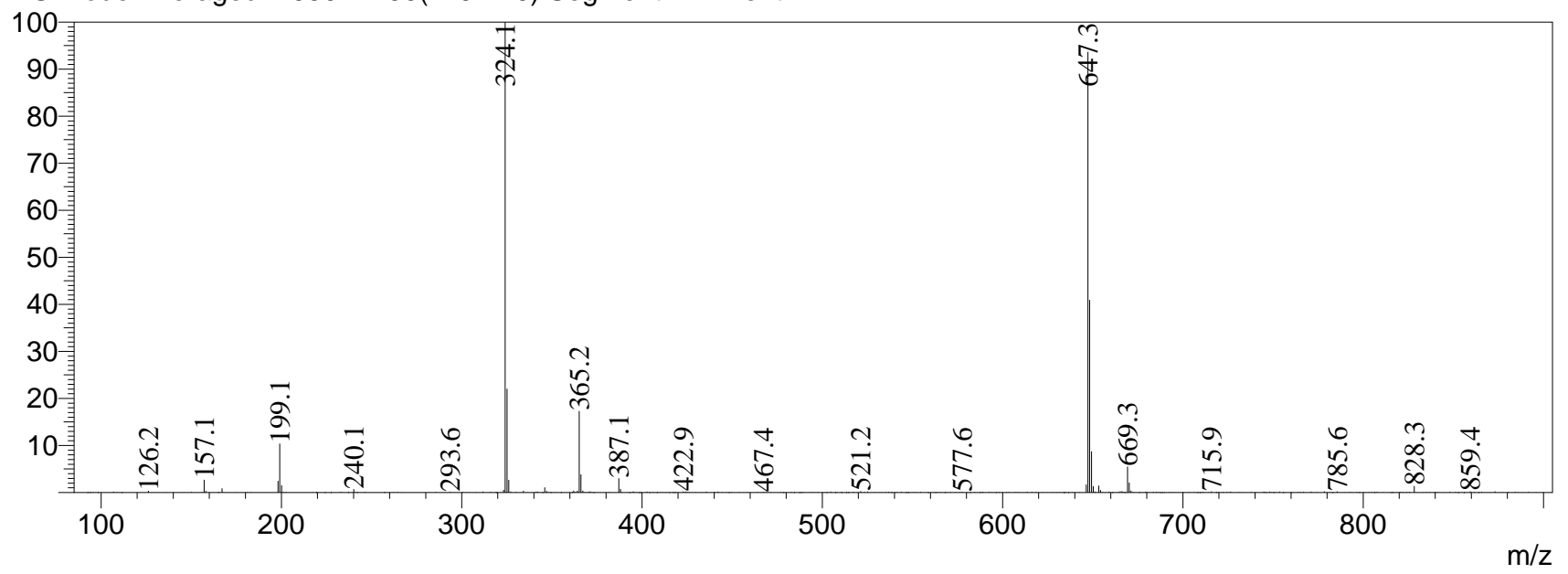


Acquired by

Sample Name

Sample ID

Injection Volume

Data File Name

Method File Name

Report File Name

Data Acquired

Data Processed

Column

Size

Mobile phase

Pressure

Flow

Instrument

Detector

Temperature
Chiral HPLC

: System Administrator

: CHIRAL_HPLC-PH-CYT-A-905-0-1(56385-136Q1)1T

: 5 uL

: PDA1601170.Icd

: Chiral.Icm

: DEFAULT.Isr

: 2016-1-25 16:46:28

: 2016-1-25 16:59:57

:Chiral Cellulose-SB

$: 0.46 * 15 \mathrm{~cm} ; 3 \mathrm{um}$

$: \mathrm{Hex}(0.1 \% \mathrm{DEA}): \mathrm{EtOH}=50: 50$

$: \mathrm{MPa}$

$: 1.0 \mathrm{ml} / \mathrm{min}$

:LC-37

:UV-254nm

$: 25^{\circ} \mathrm{C}$

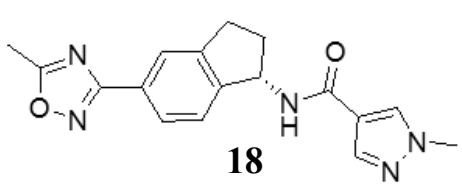

Chromatogram

CHIRAL_HPLC-PH-CYT-A-905-0-1(56385-136Q1)1T D:ILC37data\2016\201601\PDA1601170.lcd

uAU

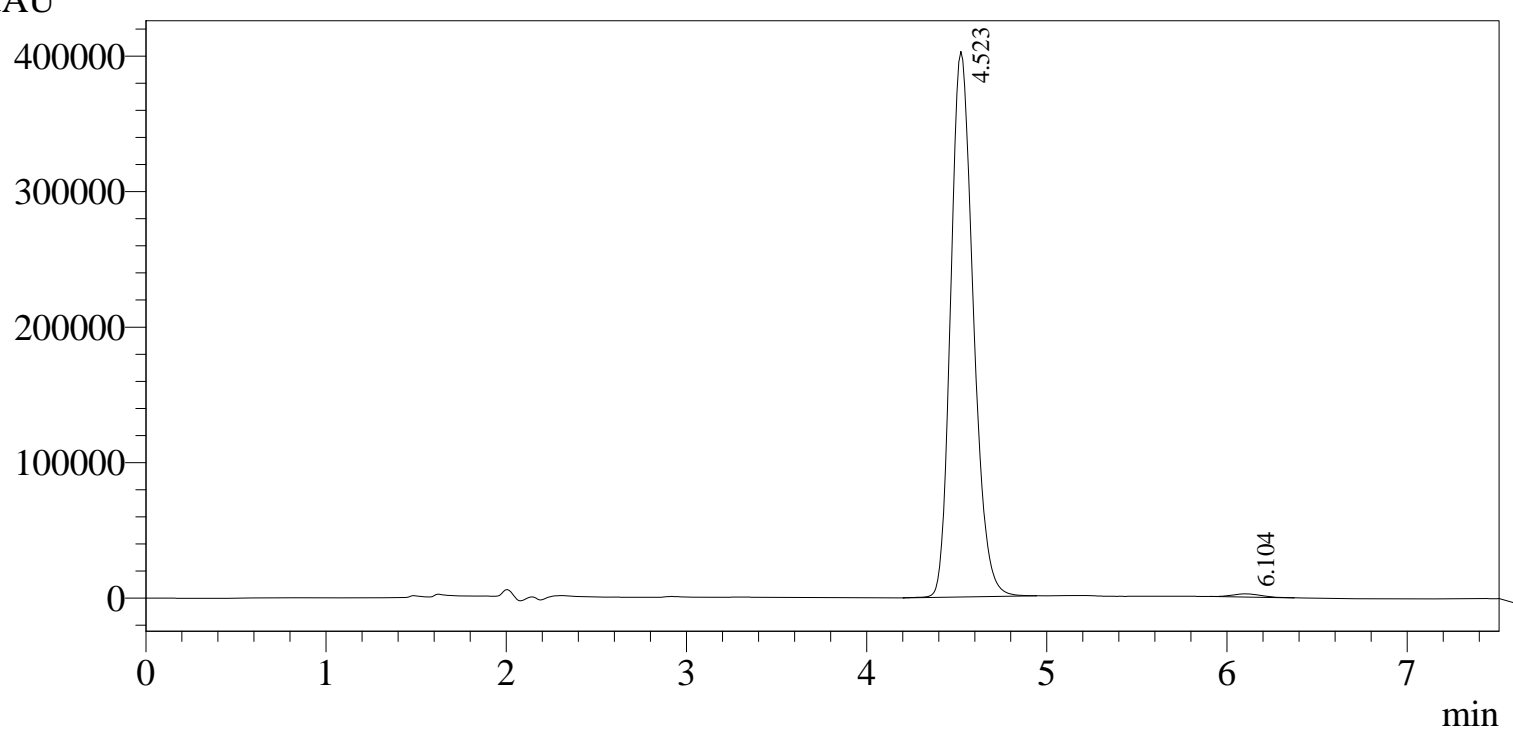

1 PDA Multi 1 / 220nm,4nm

PeakTable

PDA Ch1 220nm

\begin{tabular}{|r|r|r|r|r|r|}
\hline \multicolumn{1}{|c|}{ Peak\# } & \multicolumn{1}{|c|}{ Ret. Time } & \multicolumn{1}{c|}{ Height } & \multicolumn{1}{c|}{ Area } & \multicolumn{1}{c|}{ Height \% } & \multicolumn{1}{c|}{ Area \% } \\
\hline 1 & 4.523 & 402591 & 3507440 & 99.404 & 99.244 \\
\hline 2 & 6.104 & 2415 & 26701 & 0.596 & 0.756 \\
\hline Total & & 405006 & 3534140 & 100.000 & 100.000 \\
\hline
\end{tabular}




\section{LCMS Analysis Report}

\author{
Acquired by \\ Sample Name \\ Injection Volume \\ Data File \\ Method File \\ Report Format File \\ Date Acquired \\ Comment
}

: System Administrator

: LCMS52-PH-CYT-A-905-0-1(56385-136Q1)1T

$: 1$

: LCMS52-PH-CYT-A-905-0-1(56385-136Q1)1T.Icd

: ACN-Water-H2O-10\%B-1.3-3.0min(90-900).Icm

: LCMS2020-PDA+ELSD+TIC+MS.Isr

: 1/25/2016 14:38:16

: Mobile phaseA:water/5mM NH4HCO3;

Mobile phaseB:Acetonitrile
Instrument Name:Shimadzu LCMS-2020

$<<$ Pump $>>$

Mode

Pump A

Pump B

Total Flow

B Conc.

$<<$ Oven $>>$

Oven Temperature

$<<$ PDA $>>$

PDA Model

Lamp

Start Wavelength

End Wavelength
: Binary gradient

: LC-20ADXR

: LC-20ADXR

: $1.3000 \mathrm{~mL} / \mathrm{min}$

: $10.0 \%$

$: 40 \mathrm{C}$
$:$ SPD-M20A
$:$ D2
$: 190 \mathrm{~nm}$
$: 400 \mathrm{~nm}$

<<Interface>>
Interface
DL Temperature
Nebulizing Gas Flow
Heat Block
Drying Gas
<<MS Parameter>>
--Segment 1 Event 1--
Start Time
End Time
Acquisition Mode
Polarity
Event Time
Detector Voltage
Threshold
Start m/z
End m/z
Scan Speed
Interface Volt.
DL Volt.
Qarray DC Voltage
Qarray DC Voltage

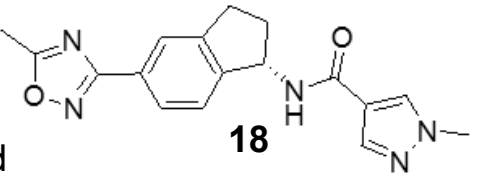

Chemical Formula: $\mathrm{C}_{17} \mathrm{H}_{17} \mathrm{~N}_{5} \mathrm{O}_{2}$ Exact Mass: 323.1382 Molecular Weight: 323.3492

\author{
:ESI \\ :250 C \\ $: 1.50 \mathrm{~L} / \mathrm{min}$ \\ $: 250 \mathrm{C}$ \\ :On \\ $15.00 \mathrm{~L} / \mathrm{min}$
}

$: 0.00 \mathrm{~min}$

:3.00 min

:Scan

:Positive

$: 0.60 \mathrm{sec}$

:+0.90 kV

$: 0$

$: 90.00$

:900.00

:1364 u/sec

:Use the Data in the Tuning File

:Use the Data in the Tuning File

:Use the Data in the Tuning File

:Use the Data in the Tuning File
System Configuration

$<<$ Column $>>$

Column Name

Length

Internal Diameter

Description

$<<$ LC Time Program $>>$
Time
0.01
2.10
2.70
2.75
3.00

\author{
: Kinetex EVO C18 100A \\ : $50 \mathrm{~mm}$ \\ : $3.0 \mathrm{~mm}$ \\ : 2.6 um
}

$\begin{array}{lll}\text { Module } & \text { Command } & \text { Value } \\ \text { Pumps } & \text { Pump B Conc. } & 10 \\ \text { Pumps } & \text { Pump B Conc. } & 95 \\ \text { Pumps } & \text { Pump B Conc. } & 95 \\ \text { Pumps } & \text { Pump B Conc. } & 10 \\ \text { Controller } & \text { Stop } & \end{array}$


mAU
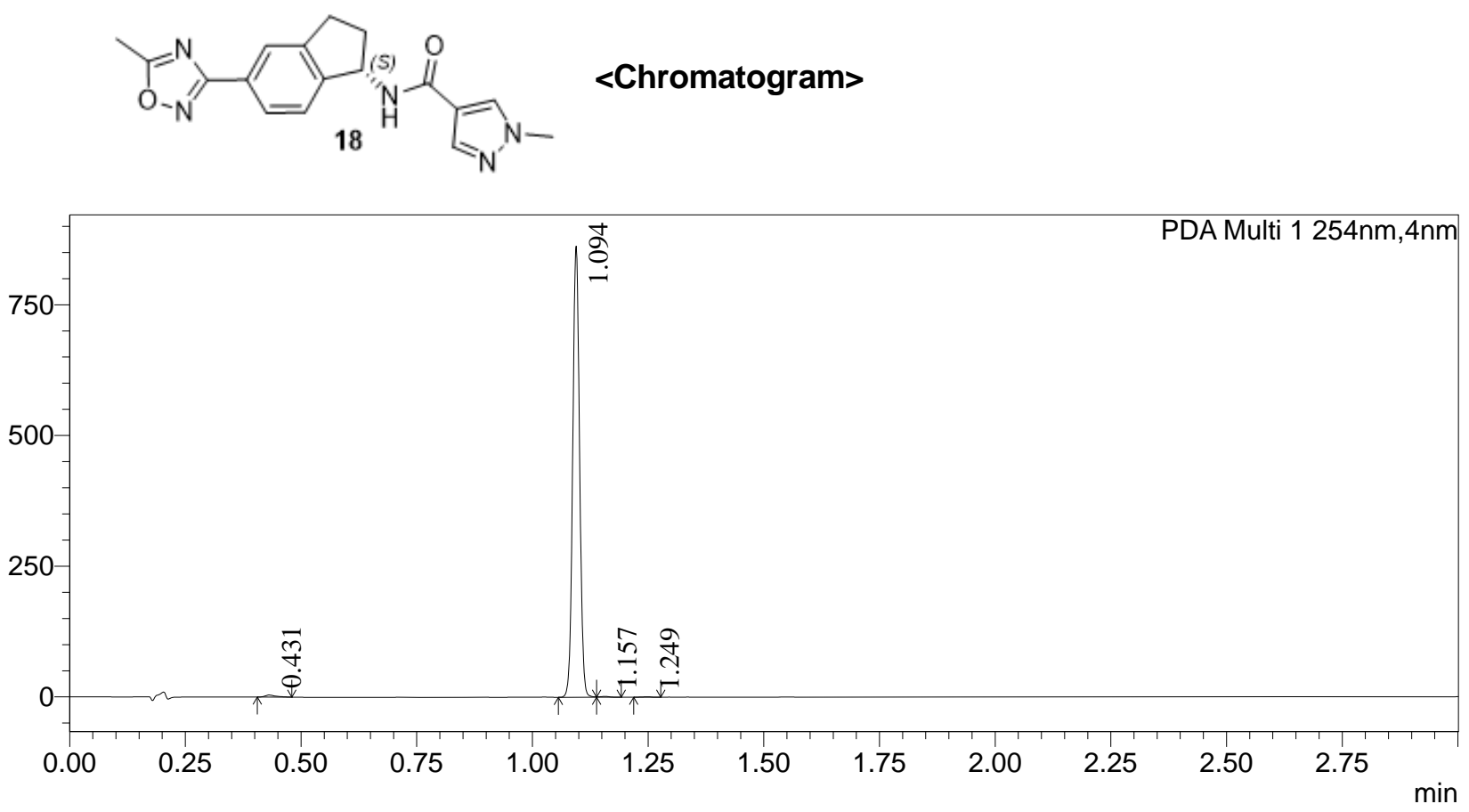

Peak Table

PDA Ch1 254nm
\begin{tabular}{|r|r|r|r|r|r|}
\hline Peak\# & Ret. Time & Height & Height $\%$ & Area & Area $\%$ \\
\hline 1 & 0.431 & 3934 & 0.459 & 6291 & 0.698 \\
\hline 2 & 1.094 & 850612 & 99.260 & 891104 & 98.944 \\
\hline 3 & 1.157 & 1561 & 0.182 & 2187 & 0.243 \\
\hline 4 & 1.249 & 848 & 0.099 & 1033 & 0.115 \\
\hline Total & & 856955 & 100.000 & 900615 & 100.000 \\
\hline
\end{tabular}

mAU

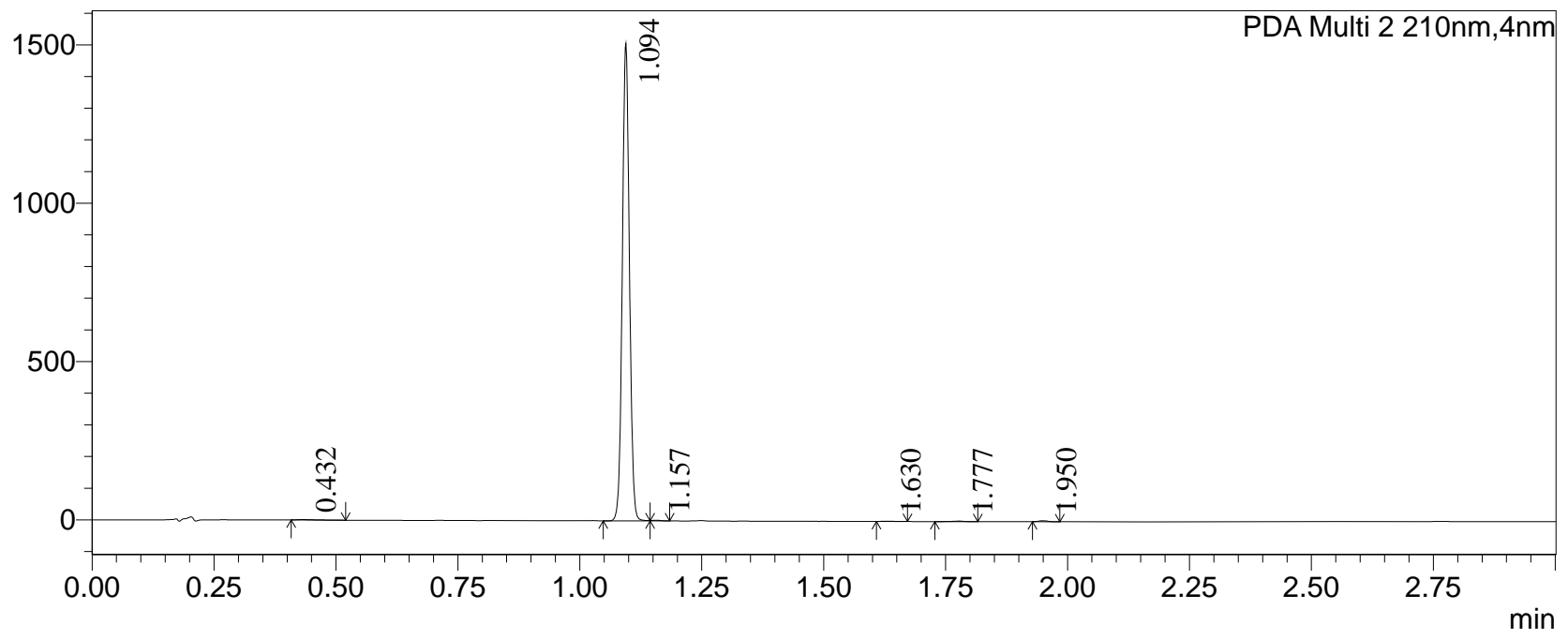

Peak Table

PDA Ch2 210nm

\begin{tabular}{|r|r|r|r|r|r|}
\hline \multicolumn{1}{|c|}{ Peak\# } & \multicolumn{1}{|c|}{ Ret. Time } & \multicolumn{1}{c|}{ Height } & Height\% & \multicolumn{1}{c|}{ Area } & \multicolumn{1}{c|}{ Area\% } \\
\hline 1 & 0.432 & 1276 & 0.091 & 2971 & 0.191 \\
\hline 2 & 1.094 & 1386201 & 99.385 & 1539359 & 99.137 \\
\hline 3 & 1.157 & 1867 & 0.134 & 2126 & 0.137 \\
\hline 4 & 1.630 & 815 & 0.058 & 1201 & 0.077 \\
\hline 5 & 1.777 & 1498 & 0.107 & 2716 & 0.175 \\
\hline 6 & 1.950 & 3126 & 0.224 & 4386 & 0.282 \\
\hline Total & & 1394783 & 100.000 & 1552759 & 100.000 \\
\hline
\end{tabular}


$\mathrm{mV}$

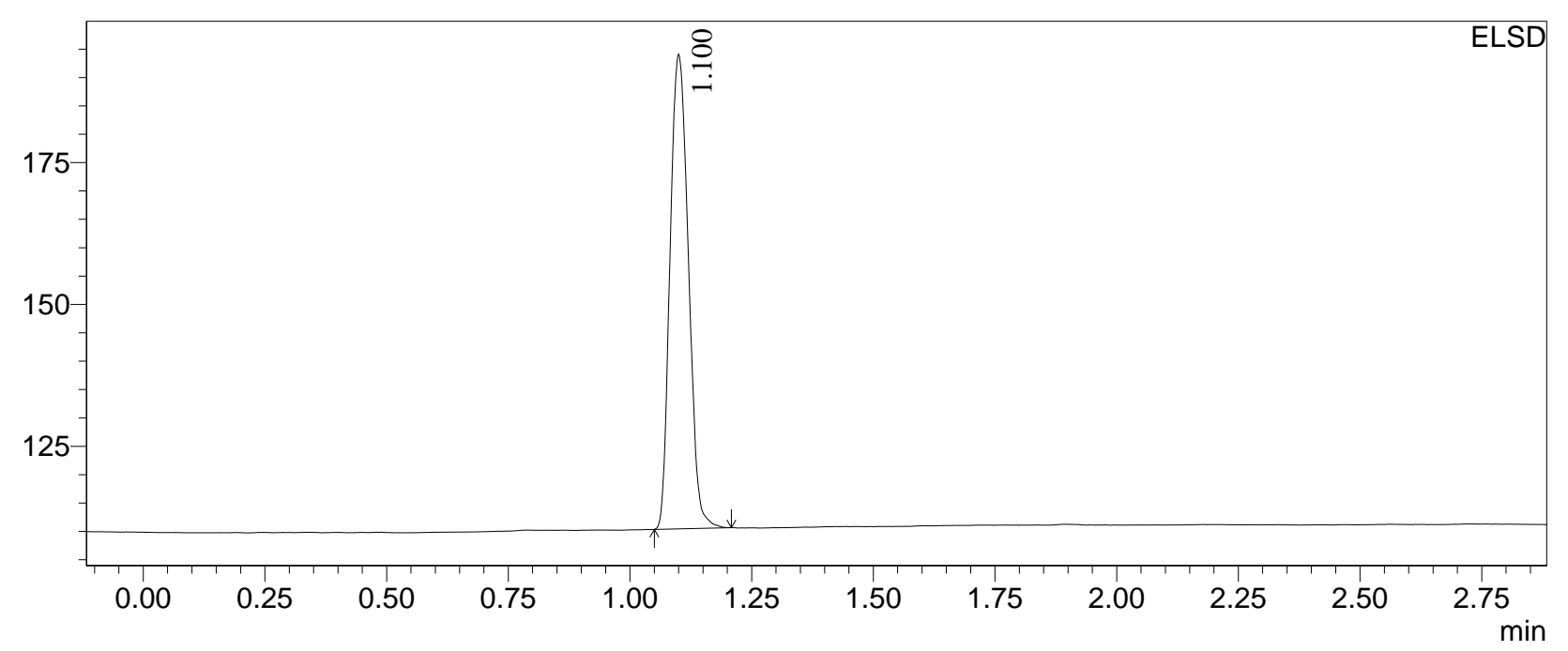

Peak Table

ELSD

\begin{tabular}{|r|r|r|r|r|r|}
\hline Peak\# & Ret. Time & Height & \multicolumn{1}{c|}{ Height\% } & \multicolumn{1}{c|}{ Area } & \multicolumn{1}{c|}{ Area\% } \\
\hline 1 & 1.100 & 81708 & 100.000 & 219670 & 100.000 \\
\hline Total & & 81708 & 100.000 & 219670 & 100.000 \\
\hline
\end{tabular}

\section{Line\#:1 R.Time:1.090(Scan\#:114)}

Mass Spectrum

\section{MassPeaks:360}

Spectrum Mode:Single 1.090(114) BasePeak:647.3(393907)

BG Mode:Averaged 1.070-1.160(112-121) Segment 1 - Event 1

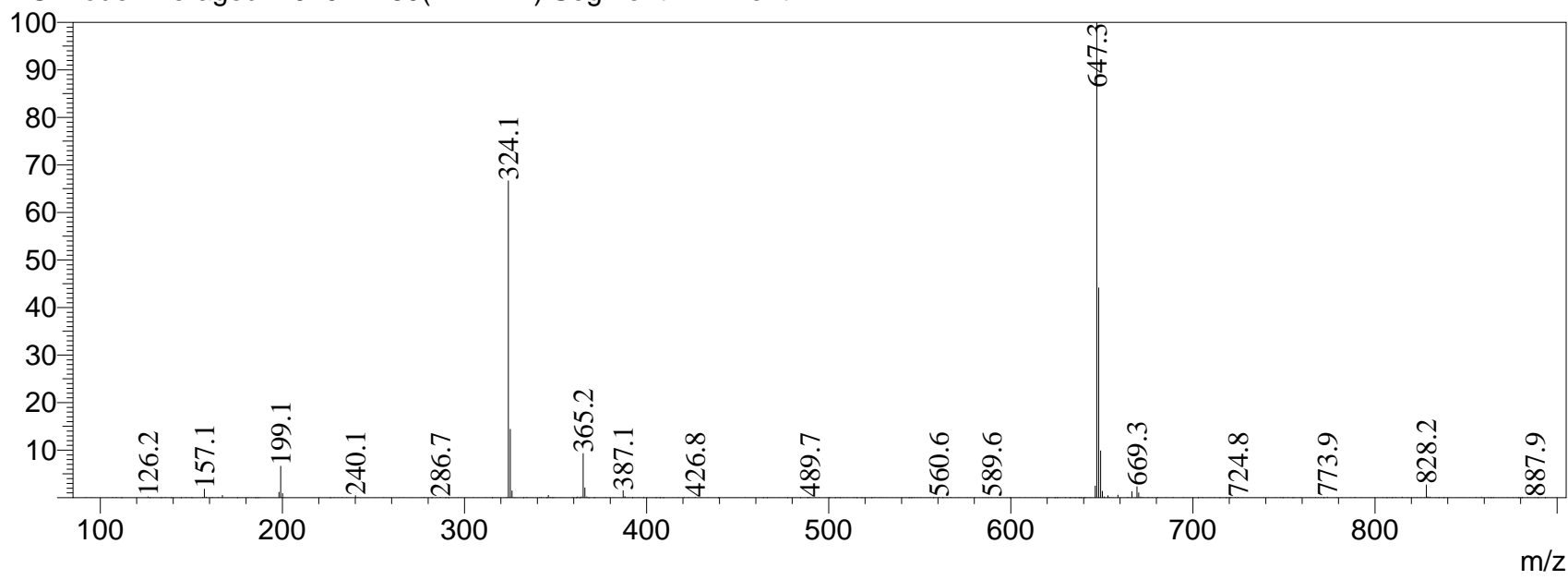




\section{LCMS Analysis Report}

\author{
Acquired by \\ Sample Name \\ Injection Volume \\ Data File \\ Method File \\ Report Format File \\ Date Acquired \\ Comment
}

: System Administrator

: LCMS52-PH-CYT-A-883-0-3(57137-157A1)1T

$: 4$

: LCMS52-PH-CYT-A-883-0-3(57137-157A1)1T.Icd

: ACN-Water-H2O-10\%B-1.3-3.0min(90-900).Icm

: LCMS2020-PDA+ELSD+TIC+MS.Isr

: 1/20/2016 15:33:05

: Mobile phaseA:water/5mM NH4HCO3; Mobile phaseB:Acetonitrile

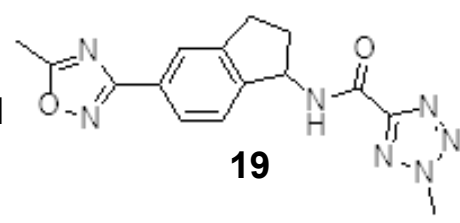

Che mical Formula: $\mathrm{C}_{15} \mathrm{H}_{15} \mathrm{~N}_{7} \mathrm{O}_{2}$ Exact Mass: 325.13

Molecular Weight: 325.33
Instrument Name:Shimadzu LCMS-2020

$<<$ Pump $>>$

Mode

Pump A

Pump B

Total Flow

B Conc.

$<<$ Oven $>>$

Oven Temperature

$<<$ PDA $>>$

PDA Model

Lamp

Start Wavelength

End Wavelength
: Binary gradient

: LC-20ADXR

: LC-20ADXR

: $1.3000 \mathrm{~mL} / \mathrm{min}$

: $10.0 \%$

$: 40 \mathrm{C}$
$:$ SPD-M20A
$:$ D2
$: 190 \mathrm{~nm}$
$: 400 \mathrm{~nm}$

<<Interface>>
Interface
DL Temperature
Nebulizing Gas Flow
Heat Block
Drying Gas
<<MS Parameter>>
--Segment 1 Event 1--
Start Time
End Time
Acquisition Mode
Polarity
Event Time
Detector Voltage
Threshold
Start m/z
End m/z
Scan Speed
Interface Volt.
DL Volt.
Qarray DC Voltage
Qarray DC Voltage

:ESI

$: 250 \mathrm{C}$

:1.50 L/min

:250 C

:On

$15.00 \mathrm{~L} / \mathrm{min}$

$: 0.00 \mathrm{~min}$

:3.00 min

:Scan

:Positive

$: 0.60 \mathrm{sec}$

:+0.90 kV

$: 0$

$: 90.00$

:900.00

:1364 u/sec

:Use the Data in the Tuning File

:Use the Data in the Tuning File

:Use the Data in the Tuning File

:Use the Data in the Tuning File
System Configuration

$<<$ Column $>>$

Column Name

Length

Internal Diameter

Description

$<<$ LC Time Program $>>$
Time
0.01
2.10
2.70
2.75
3.00

\author{
: Kinetex EVO C18 100A \\ : $50 \mathrm{~mm}$ \\ : $3.0 \mathrm{~mm}$ \\ : 2.6 um
}

$\begin{array}{lll}\text { Module } & \text { Command } & \text { Value } \\ \text { Pumps } & \text { Pump B Conc. } & 10 \\ \text { Pumps } & \text { Pump B Conc. } & 95 \\ \text { Pumps } & \text { Pump B Conc. } & 95 \\ \text { Pumps } & \text { Pump B Conc. } & 10 \\ \text { Controller } & \text { Stop } & \end{array}$


mAU
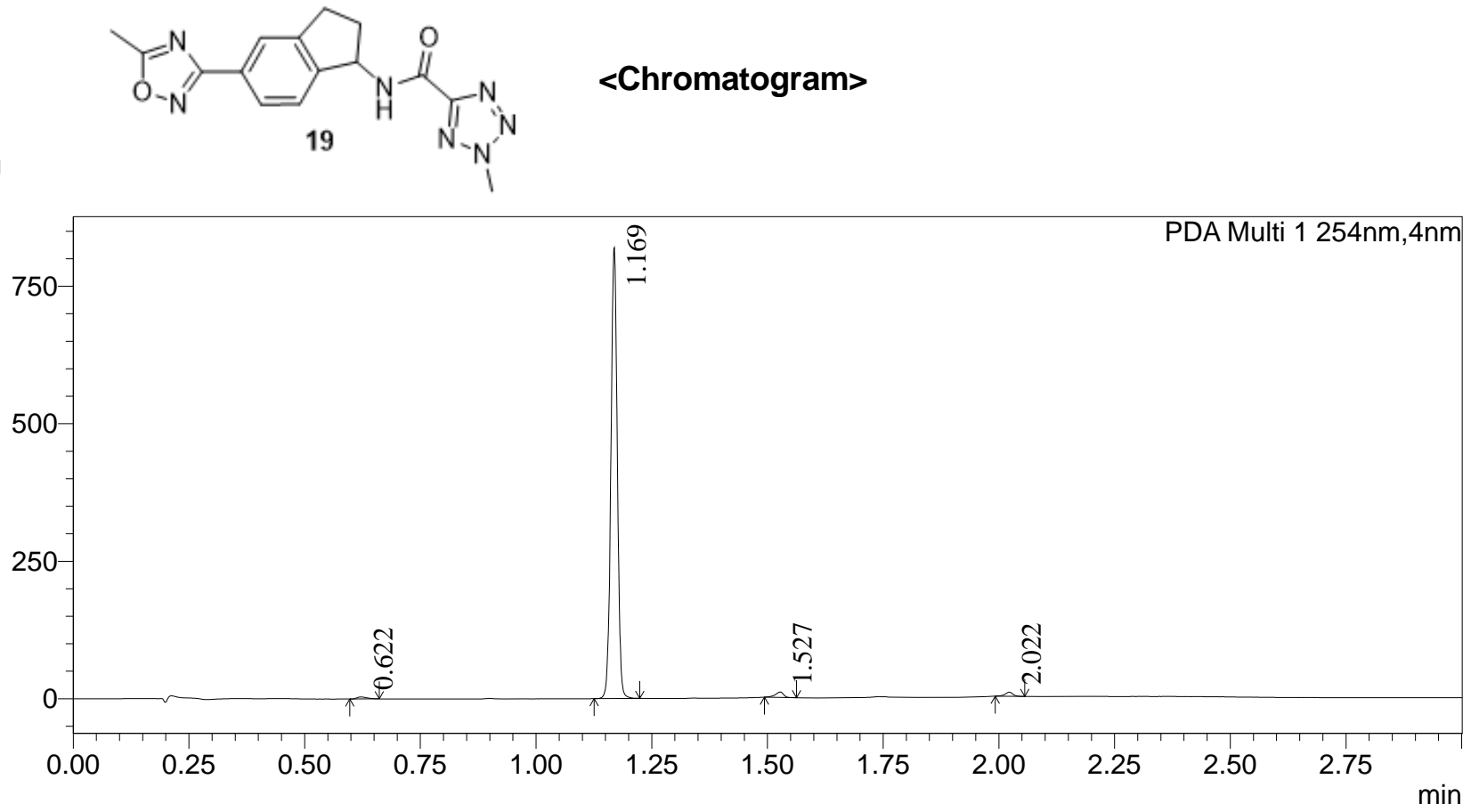

Peak Table

PDA Ch1 254nm
\begin{tabular}{|r|r|r|r|r|r|}
\hline Peak\# & Ret. Time & \multicolumn{1}{|c|}{ Height } & Height $\%$ & \multicolumn{1}{c|}{ Area } & \multicolumn{1}{|c|}{ Area $\%$} \\
\hline 1 & 0.622 & 3678 & 0.447 & 5819 & 0.712 \\
\hline 2 & 1.169 & 802217 & 97.440 & 789990 & 96.652 \\
\hline 3 & 1.527 & 9899 & 1.202 & 12656 & 1.548 \\
\hline 4 & 2.022 & 7501 & 0.911 & 8885 & 1.087 \\
\hline Total & & 823296 & 100.000 & 817352 & 100.000 \\
\hline
\end{tabular}

mAU

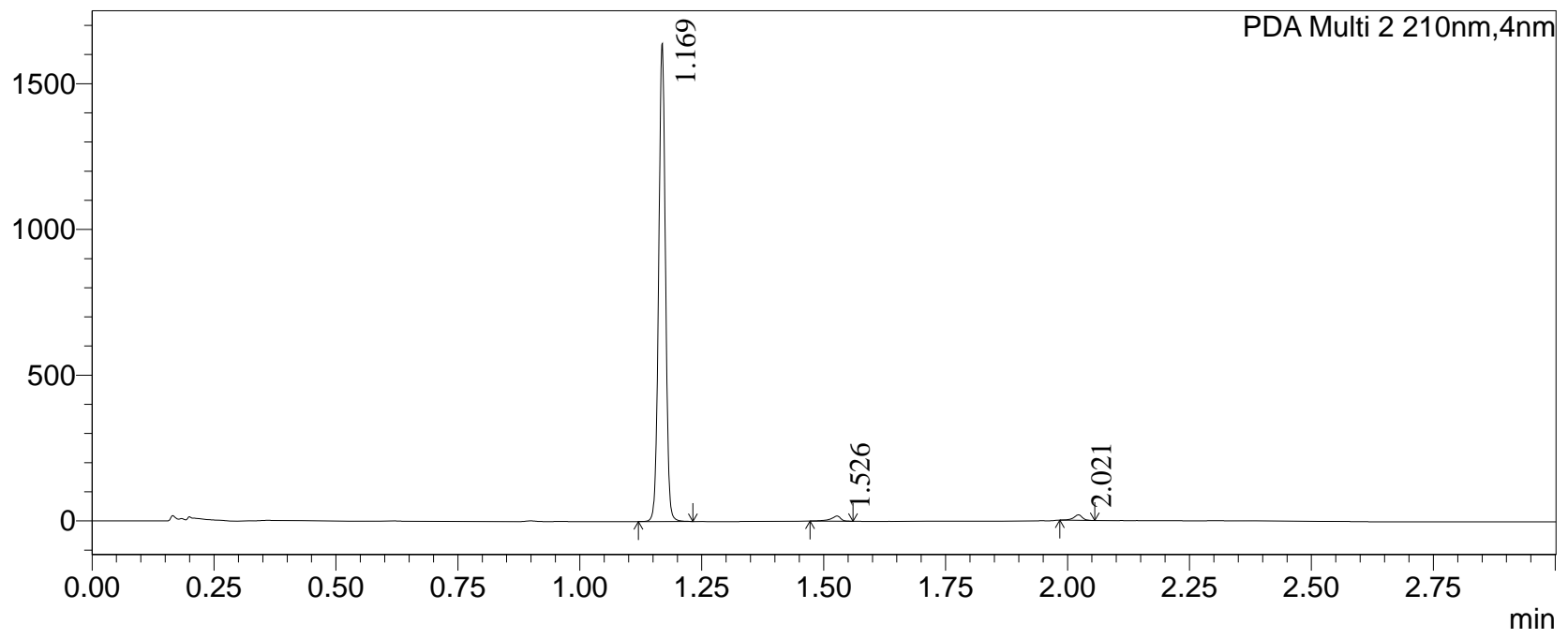

Peak Table

PDA Ch2 210nm

\begin{tabular}{|r|r|r|r|r|r|}
\hline Peak\# & \multicolumn{1}{|c|}{ Ret. Time } & \multicolumn{1}{c|}{ Height } & Height\% & \multicolumn{1}{c|}{ Area } & \multicolumn{1}{c|}{ Area\% } \\
\hline 1 & 1.169 & 1278967 & 97.405 & 1589675 & 97.014 \\
\hline 2 & 1.526 & 16098 & 1.226 & 25177 & 1.537 \\
\hline 3 & 2.021 & 17978 & 1.369 & 23744 & 1.449 \\
\hline Total & & 1313042 & 100.000 & 1638596 & 100.000 \\
\hline
\end{tabular}


$\mathrm{mV}$

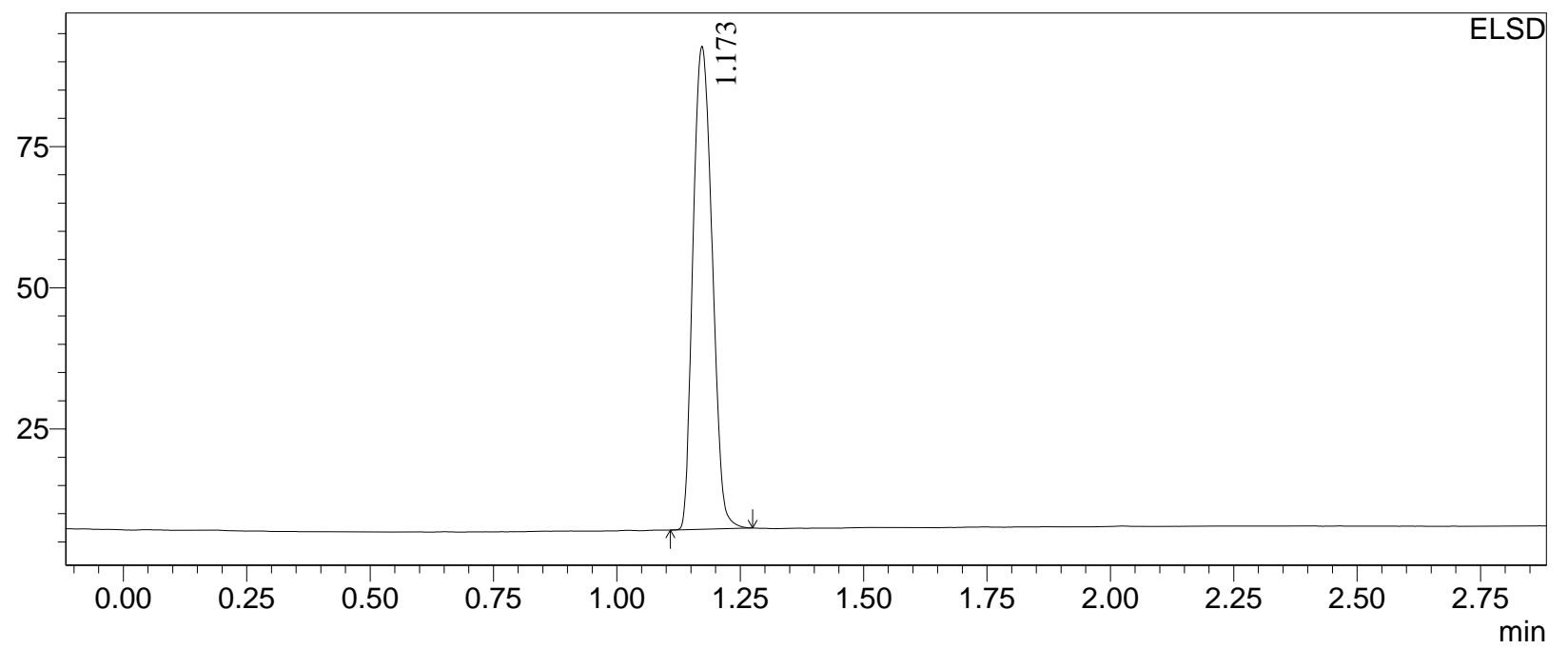

Peak Table

ELSD

\begin{tabular}{|r|r|r|r|r|r|}
\hline Peak\# & Ret. Time & Height & \multicolumn{1}{c|}{ Height\% } & \multicolumn{1}{c|}{ Area } & \multicolumn{1}{c|}{ Area\% } \\
\hline 1 & 1.173 & 84866 & 100.000 & 231484 & 100.000 \\
\hline Total & & 84866 & 100.000 & 231484 & 100.000 \\
\hline
\end{tabular}

Line\#:1 R.Time:1.170(Scan\#:122)

Mass Spectrum

MassPeaks:380

Spectrum Mode:Single 1.170(122) BasePeak:326.1(59259)

BG Mode:Averaged 1.120-1.210(117-126) Segment 1 - Event 1

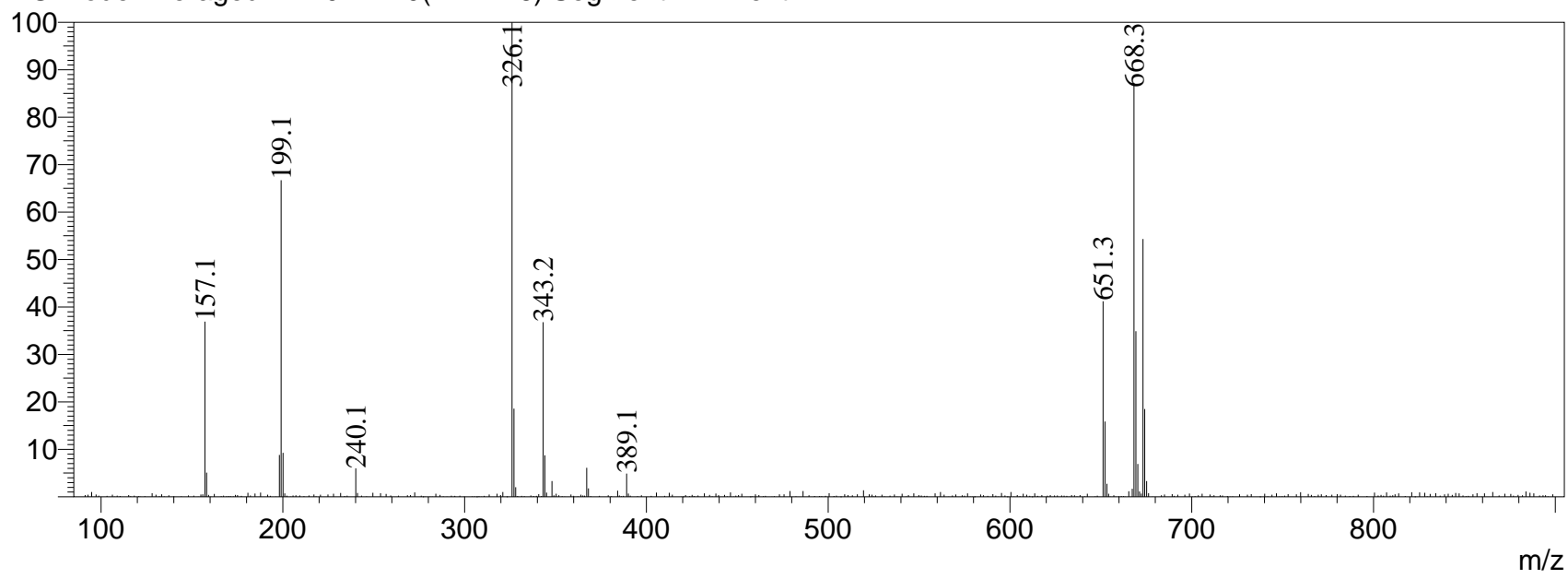




\section{LCMS Analysis Report}

\section{Acquired by Sample Name Injection Volume Data File Method File Report Format File Date Acquired Comment}

: System Administrator

: LCMS44-PH-CYT-A-895-0-2(57137-167A1)1T

$: 0.2$

: LCMS44-PH-CYT-A-895-0-2(57137-167A1)1T002.Icd

: ACN-Water-0.05\%TFA-5\%B-1.5-5.0MIN(90-900).Icm

: LCMS2020-PDA+ELS+TIC+MS.Isr

: 2016-1-25 13:20:20

: Mobile Phase A :Water+0.05\%TFA; Mobile Phase B:ACN+0.05\%TFA

Instrument Name:Shimadzu LCMS-2020

$<<$ Pump $>>$

Mode

Pump A

Pump B

Total Flow

B Conc.

$<<$ Oven $>>$

Oven Temperature

$<<$ PDA $>>$

PDA Model

Lamp

Start Wavelength

End Wavelength

<<Interface>>
Interface
DL Temperature
Nebulizing Gas Frow
Heat Block
Drying Gas
<<MS Parameter>>
Initial Valve Position
--Segment 1 Event 1--
Start Time
End Time
Acquisition Mode
Polarity
Event Time
Detector Voltage
Threshold
Start m/z
End m/z
Scan Speed
Interface Volt.
DL Volt.
Qarray DC Voltage
Qarray DC Voltage

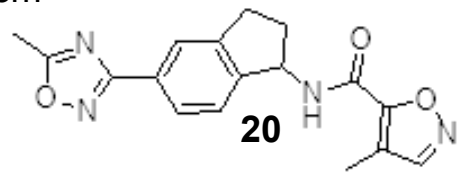

Chemical Formula: $\mathrm{C}_{17} \mathrm{H}_{18} \mathrm{~N}_{4} \mathrm{O}_{3}$ Exact Mass: 324.12 :ESI Molecular Weight: 324.33 $: 250 \mathrm{C}$ $: 1.50 \mathrm{~L} / \mathrm{min}$ $: 300 \mathrm{C}$

:On $15.00 \mathrm{~L} / \mathrm{min}$

:-

$: 0.00 \mathrm{~min}$ $: 5.00 \mathrm{~min}$

:Scan

:Positive

:0.50 sec :+0.85 kV $: 0$ $: 90.00$

:900.00

:1667 u/sec

:Use the Data in the Tuning File :Use the Data in the Tuning File :Use the Data in the Tuning File :Use the Data in the Tuning File
System Configuration $<<$ Column $>>$

Column Name

Length

Internal Diameter

Description
: Ascentis Express C18

: $50 \mathrm{~mm}$

: $3.0 \mathrm{~mm}$

: 2.7 um

$<<$ LC Time Program $>>$

$\begin{array}{ll}\text { Time } & \text { Module } \\ 0.01 & \text { Pumps } \\ 3.50 & \text { Pumps } \\ 4.60 & \text { Pumps } \\ 4.80 & \text { Pumps } \\ 5.00 & \text { Controller }\end{array}$

$\begin{array}{ll}\text { Command } & \text { Value } \\ \text { Pump B Conc. } & 5 \\ \text { Pump B Conc. } & 100 \\ \text { Pump B Conc. } & 100 \\ \text { Pump B Conc. } & 5\end{array}$

Stop 
mAU
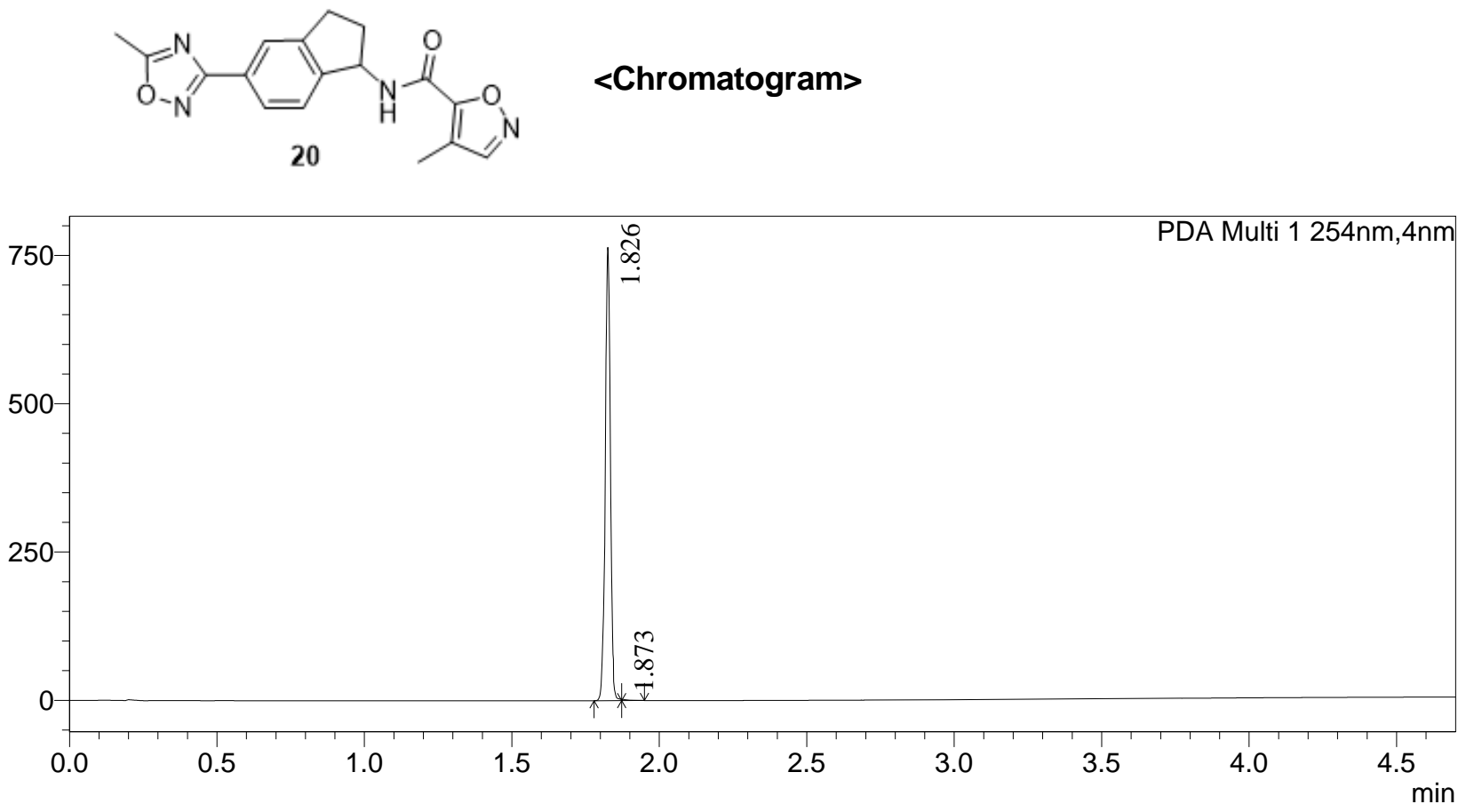

Peak Table

PDA Ch1 254nm

\begin{tabular}{|r|r|r|r|r|r|}
\hline Peak\# & Ret. Time & \multicolumn{1}{|c|}{ Height } & Height\% & \multicolumn{1}{|c|}{ Area } & \multicolumn{1}{|c|}{ Area\% } \\
\hline 1 & 1.826 & 760508 & 99.707 & 922700 & 99.666 \\
\hline 2 & 1.873 & 2239 & 0.293 & 3090 & 0.334 \\
\hline Total & & 762746 & 100.000 & 925790 & 100.000 \\
\hline
\end{tabular}

mAU

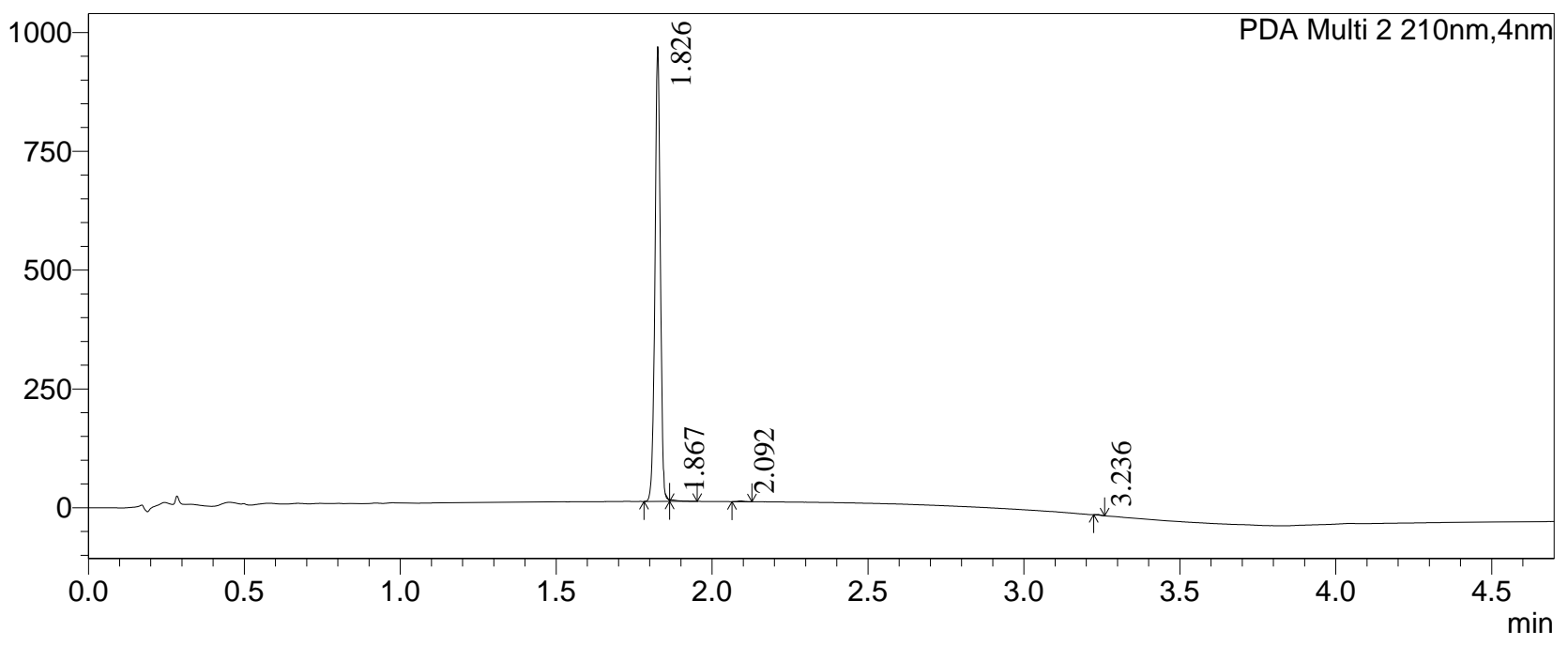

Peak Table

PDA Ch2 210nm

\begin{tabular}{|r|r|r|r|r|r|}
\hline Peak\# & Ret. Time & \multicolumn{1}{c|}{ Height } & \multicolumn{1}{c|}{ Height\% } & \multicolumn{1}{c|}{ Area } & \multicolumn{1}{c|}{ Area\% } \\
\hline 1 & 1.826 & 952324 & 99.303 & 1146333 & 99.179 \\
\hline 2 & 1.867 & 3538 & 0.369 & 5975 & 0.517 \\
\hline 3 & 2.092 & 1642 & 0.171 & 2239 & 0.194 \\
\hline 4 & 3.236 & 1509 & 0.157 & 1278 & 0.111 \\
\hline Total & & 959014 & 100.000 & 1155825 & 100.000 \\
\hline
\end{tabular}


$\mathrm{mV}$

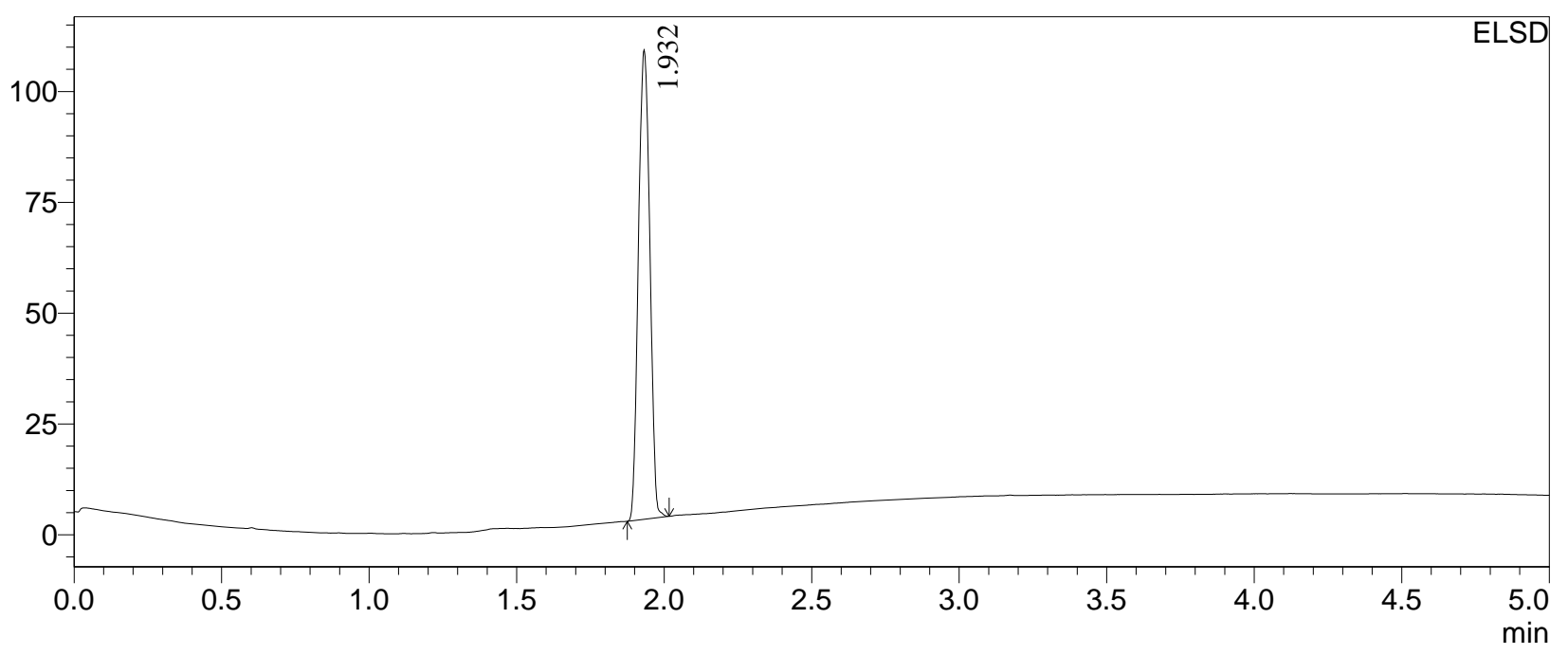

Peak Table

ELSD

\begin{tabular}{|r|r|r|r|r|r|}
\hline Peak\# & Ret. Time & Height & \multicolumn{1}{c|}{ Height\% } & \multicolumn{1}{c|}{ Area } & \multicolumn{1}{c|}{ Area\% } \\
\hline 1 & 1.932 & 104639 & 100.000 & 285165 & 100.000 \\
\hline Total & & 104639 & 100.000 & 285165 & 100.000 \\
\hline
\end{tabular}

Line\#:1 R.Time:----(Scan\#:----)

Mass Spectrum

MassPeaks:429

Spectrum Mode:Averaged 1.817-1.833(226-228) BasePeak:671.1(103798)

BG Mode:Calc Segment 1 - Event 1

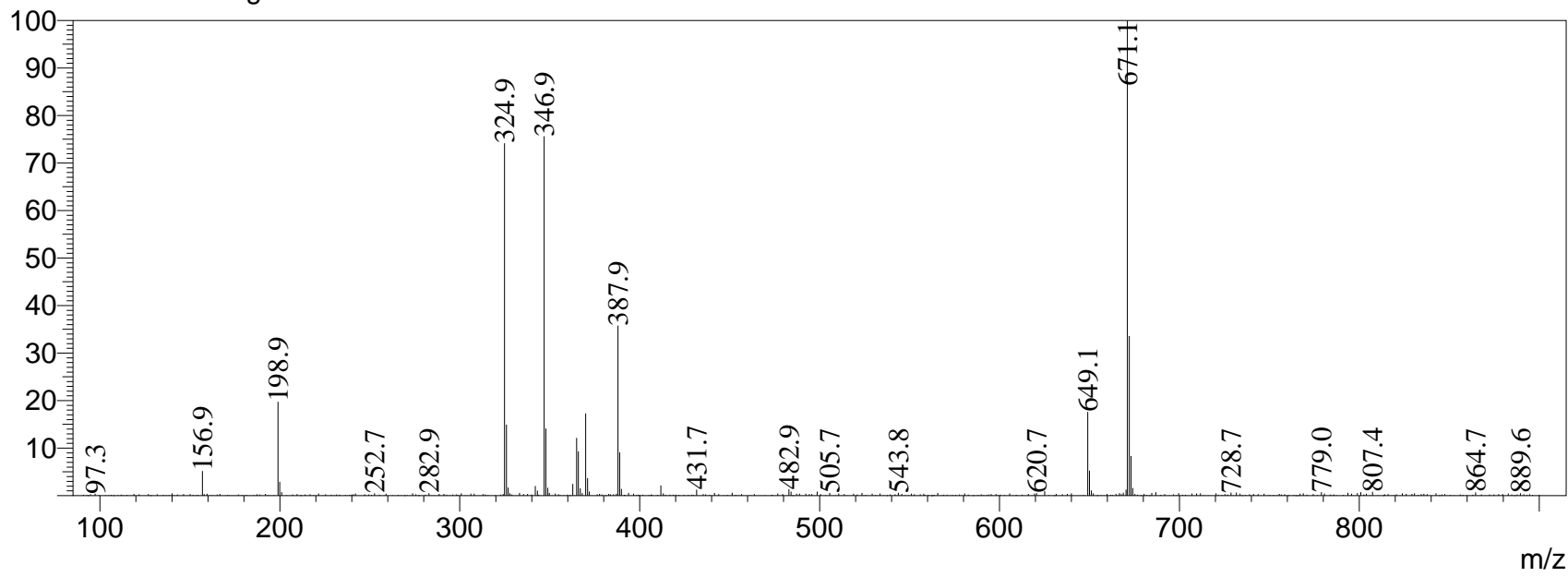


Data File D: \MassHunter\gchuang \2018-03-20\10M_POS_gc-0877-001_4222.D

Sample Name: gc-0877-001

Walkup method: '10MIN POS'
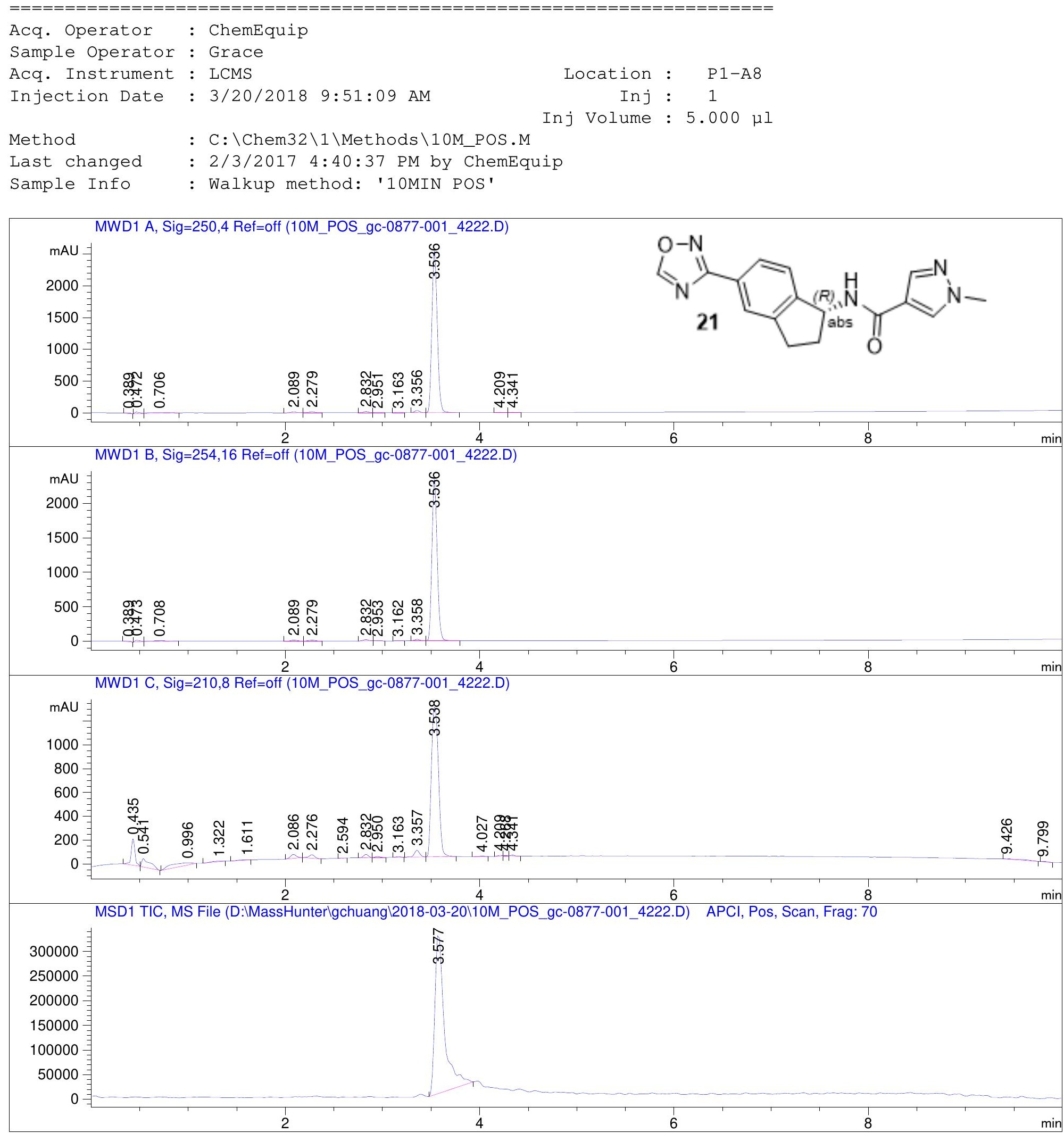
Data File D: \MassHunter\gchuang \2018-03-20\10M_POS_gC-0877-001_4222.D

Sample Name: gc-0877-001

MS Signal: MSD1 TIC, MS File, APCI, Pos, Scan, Frag: 70 Spectra averaged over upper half of peaks.

Noise Cutoff: 1000 counts.

Reportable Ion Abundance: > 10\%.

Retention

Time (MS)

3.577

2221306
Mol. Weight

or Ion

$$
311.10 \mathrm{I}
$$$$
310.10 \mathrm{I}
$$

*MSD1 SPC, time=3.551:3.624 of D:IMassHunterlgchuangl2018-03-20110M_POS_gc-0877-001_4222.D APCI, Pos, Scan, Frag: 70

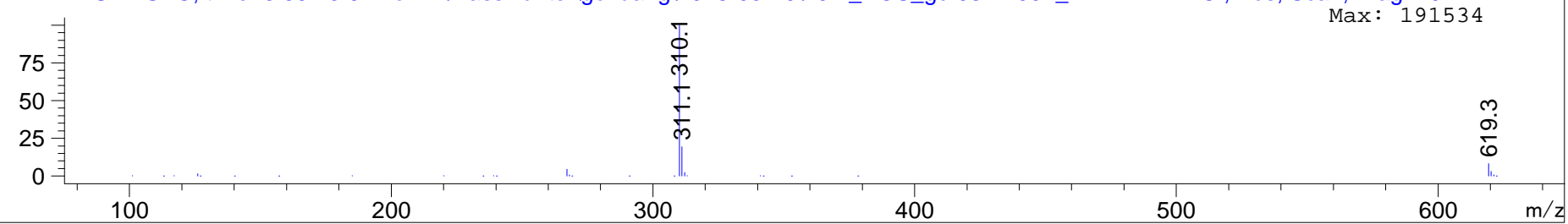




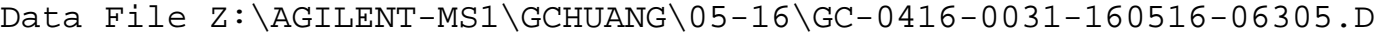

Sample Name: gc-0416-003

Easy-Access Method: '10M_POS'

\begin{tabular}{|c|c|c|c|}
\hline $\begin{array}{l}\text { Acq. Operator } \\
\text { Sample Operator }\end{array}$ & $\begin{array}{l}\text { : chemequip } \\
: \text { chemequip }\end{array}$ & & \\
\hline Acq. Instrument & : AGILENTMS1 & Location & : Vial 69 \\
\hline Injection Date & $: 5 / 16 / 20162: 56: 30 \mathrm{PM}$ & $\begin{array}{r}\operatorname{Inj} \\
j \text { Volume }\end{array}$ & $\begin{array}{ll}: & 1 \\
: & 5.000 \mu 1\end{array}$ \\
\hline Method & $: C: \backslash$ CHEM32 $22 \backslash \mathrm{METHODS} \backslash 10 \mathrm{M} \_P O S . M$ & & \\
\hline Last changed & $\begin{aligned}: & 5 / 16 / 2016 \text { 2:55:24 PM by chemequir } \\
& \text { (modified after loading) }\end{aligned}$ & & \\
\hline Sample Info & : Easy-Access Method: '10M_POS' & & \\
\hline
\end{tabular}
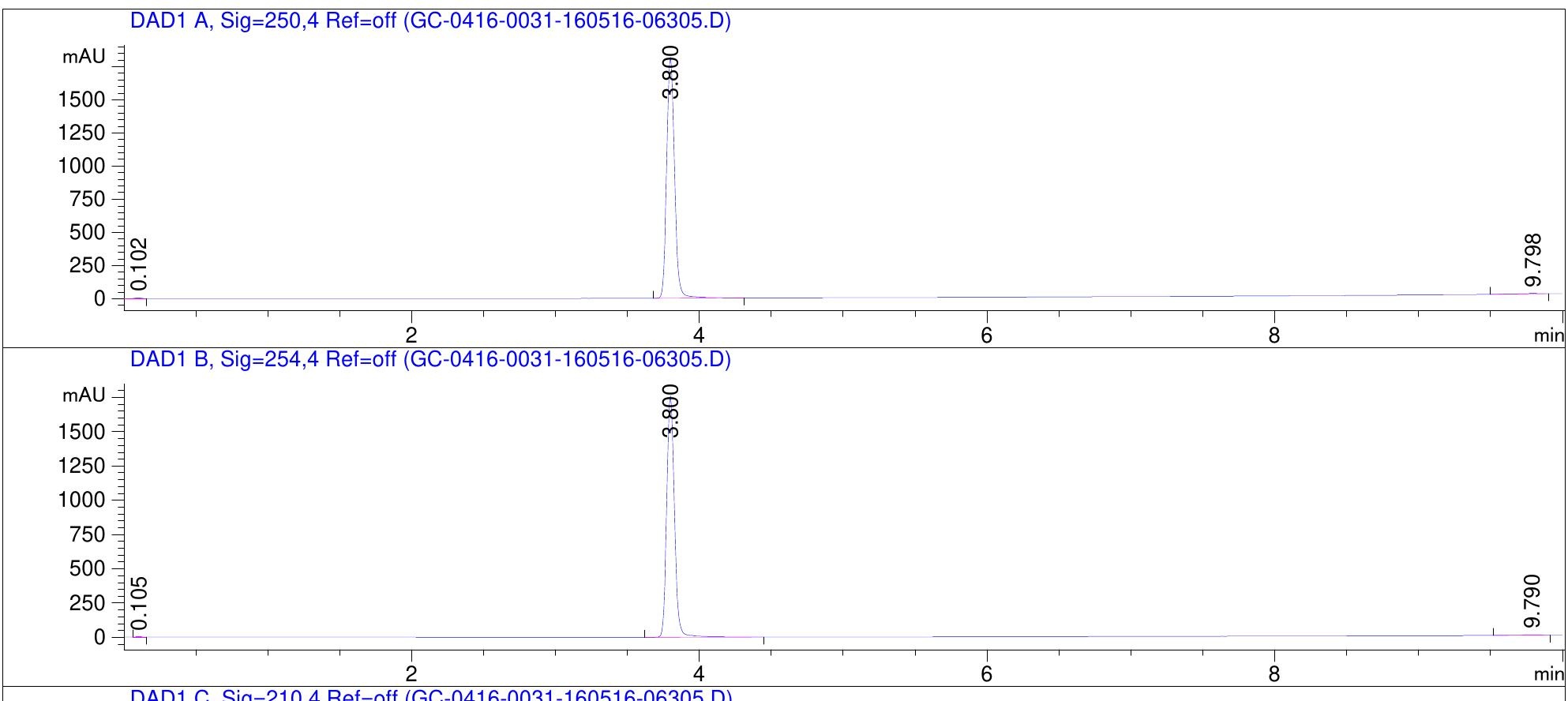

DAD1 C, Sig=210,4 Ref=off (GC-0416-0031-160516-06305.D)
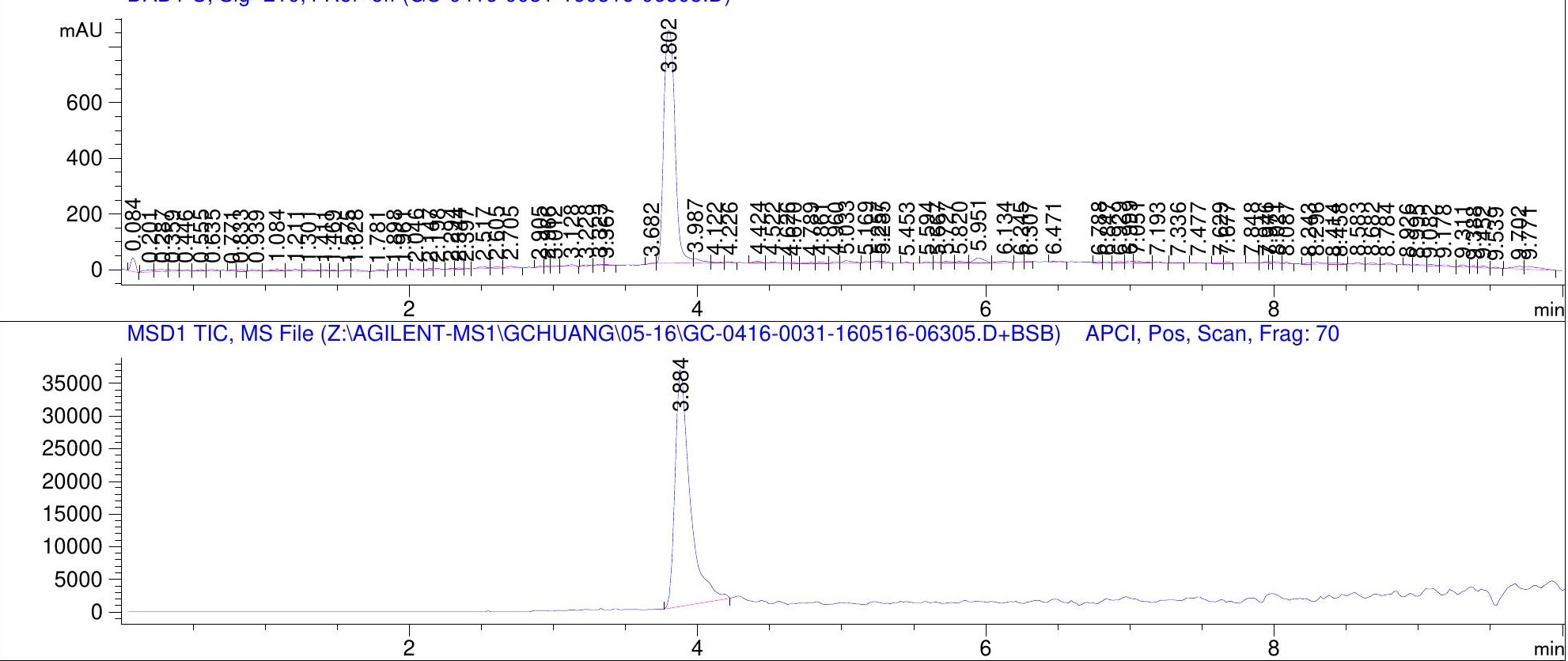
Data File Z: \AGILENT-MS1 \GCHUANG \05-16\GC-0416-0031-160516-06305.D

Sample Name: gc-0416-003

MS Signal: MSD1 TIC, MS File, APCI, Pos, Scan, Frag: 70

Subtracted background from 0.000 to $3.000 \mathrm{~min}$.

Spectra averaged over upper half of peaks.

Noise Cutoff: 1000 counts.

Reportable Ion Abundance: > 10\%.

LC Signal: DAD1 A, Sig=250,4 Ref=off

Peak matching window: $0.1 \mathrm{~min}$

$\begin{array}{crcrc}\begin{array}{c}\text { Retention } \\ \text { Time (LC) }\end{array} & \text { LC Area } & \begin{array}{c}\text { Retention } \\ \text { Time (MS) }\end{array} & \text { MS Area } & \text { Mol. Weight } \\ 0.102 & 13 & - & & \\ 3.800 & 6866 & 3.884 & 261705 & 351.20 \text { I } \\ & & & & 350.10 \text { I }\end{array}$

9.798

33

${ }^{*}$ MSD1 SPC, time=3.861:3.934 of Z:IAGILENT-MS1 Igchuangl05-16|GC-0416-0031-160516-06305.D+BSB APCI, Pos, Scan, Frag: 7

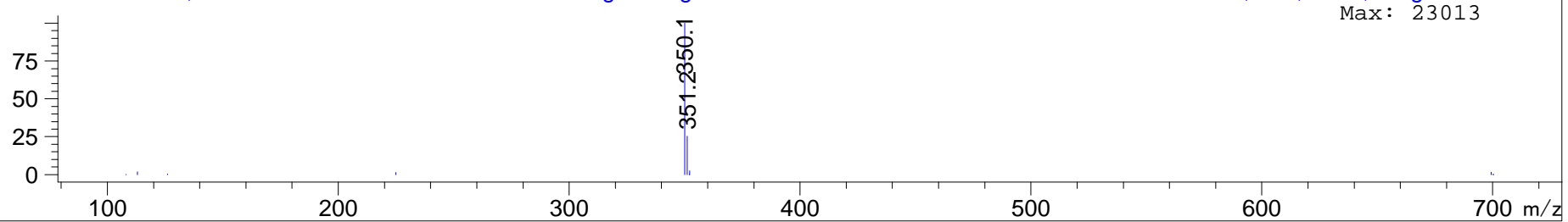


Data File Z:\AGILENT-MS1\GCHUANG\12-16\GC-0529-0011-141216-14047.D

Sample Name: gc-0529-001

Easy-Access Method: '10M_POS'

$====================$

Acq. Operator : chemequip

Sample Operator : chemequip

Acq. Instrument : AGILENTMS1

Injection Date : 12/14/2016 3:00:12 PM

Location : Vial 31

Inj : 1

Method : C : \CHEM32 $2 \backslash$ METHODS $\backslash 10 M \_P O S . M$

Last changed : 12/14/2016 2:59:06 PM by chemequip

(modified after loading)

Sample Info : Easy-Access Method: '10M_PoS'
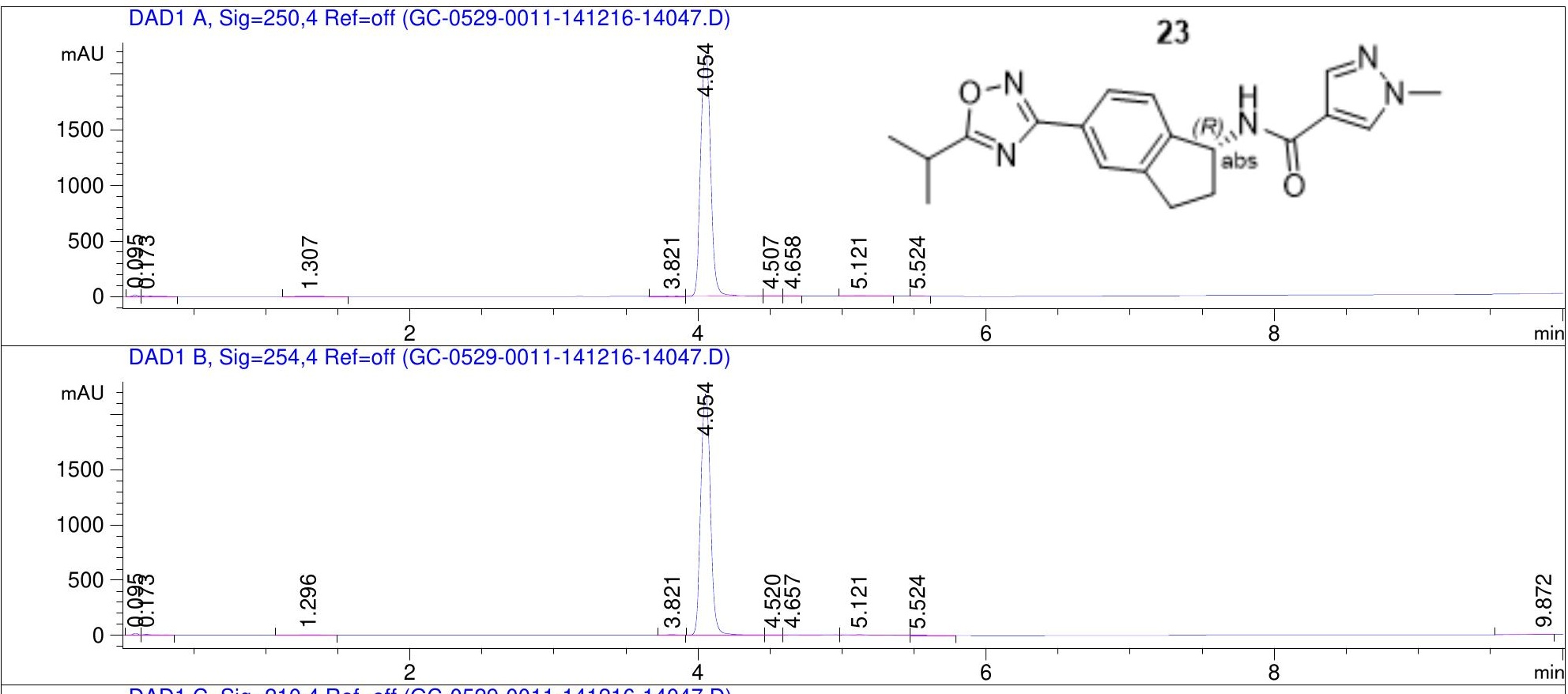

DAD1 C, Sig=210,4 Ref=off (GC-0529-0011-141216-14047.D)
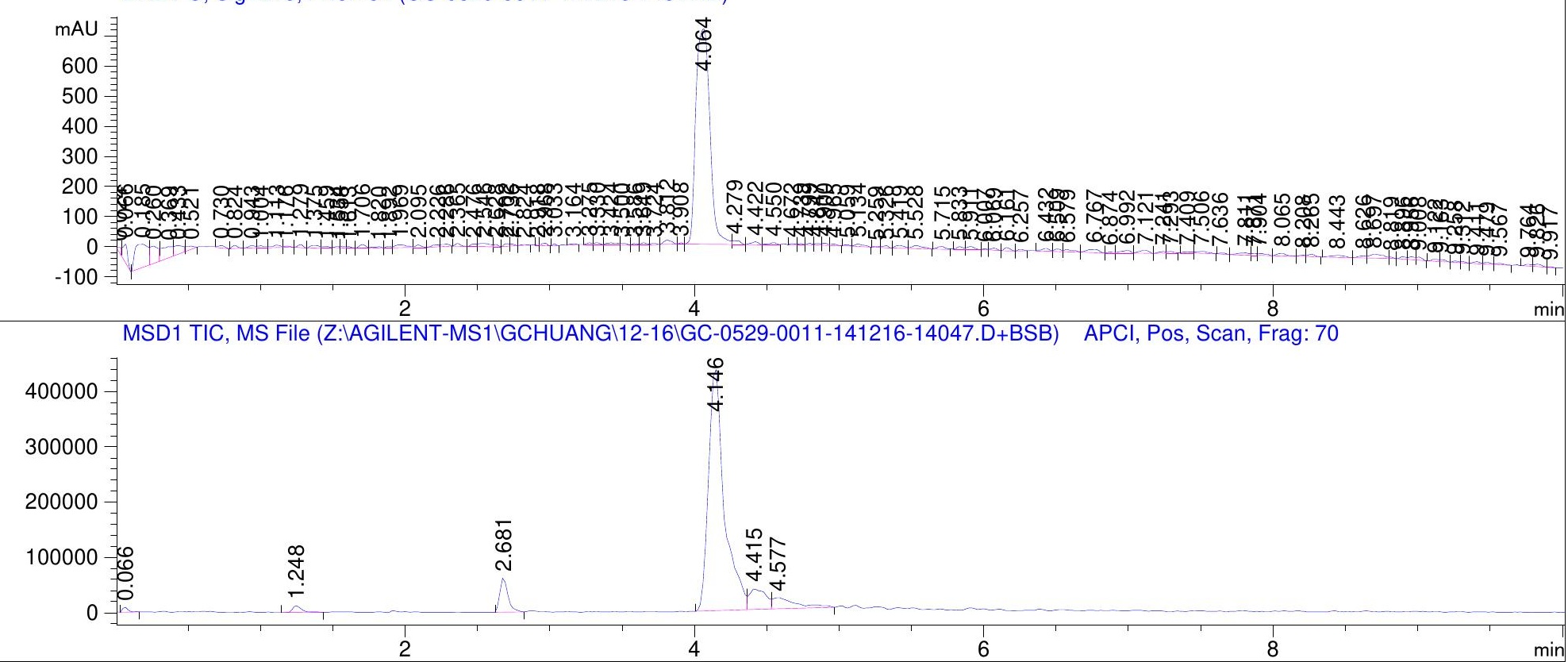
Data File Z:\AGILENT-MS1\GCHUANG\12-16\GC-0529-0011-141216-14047.D

Sample Name: gc-0529-001

MS Signal: MSD1 TIC, MS File, APCI, Pos, Scan, Frag: 70

Subtracted background from 0.000 to $3.000 \mathrm{~min}$.

Spectra averaged over upper half of peaks.

Noise Cutoff: 1000 counts.

Reportable Ion Abundance: > 10\%.

LC Signal: DAD1 A, Sig=250,4 Ref=off

Peak matching window: $0.1 \mathrm{~min}$

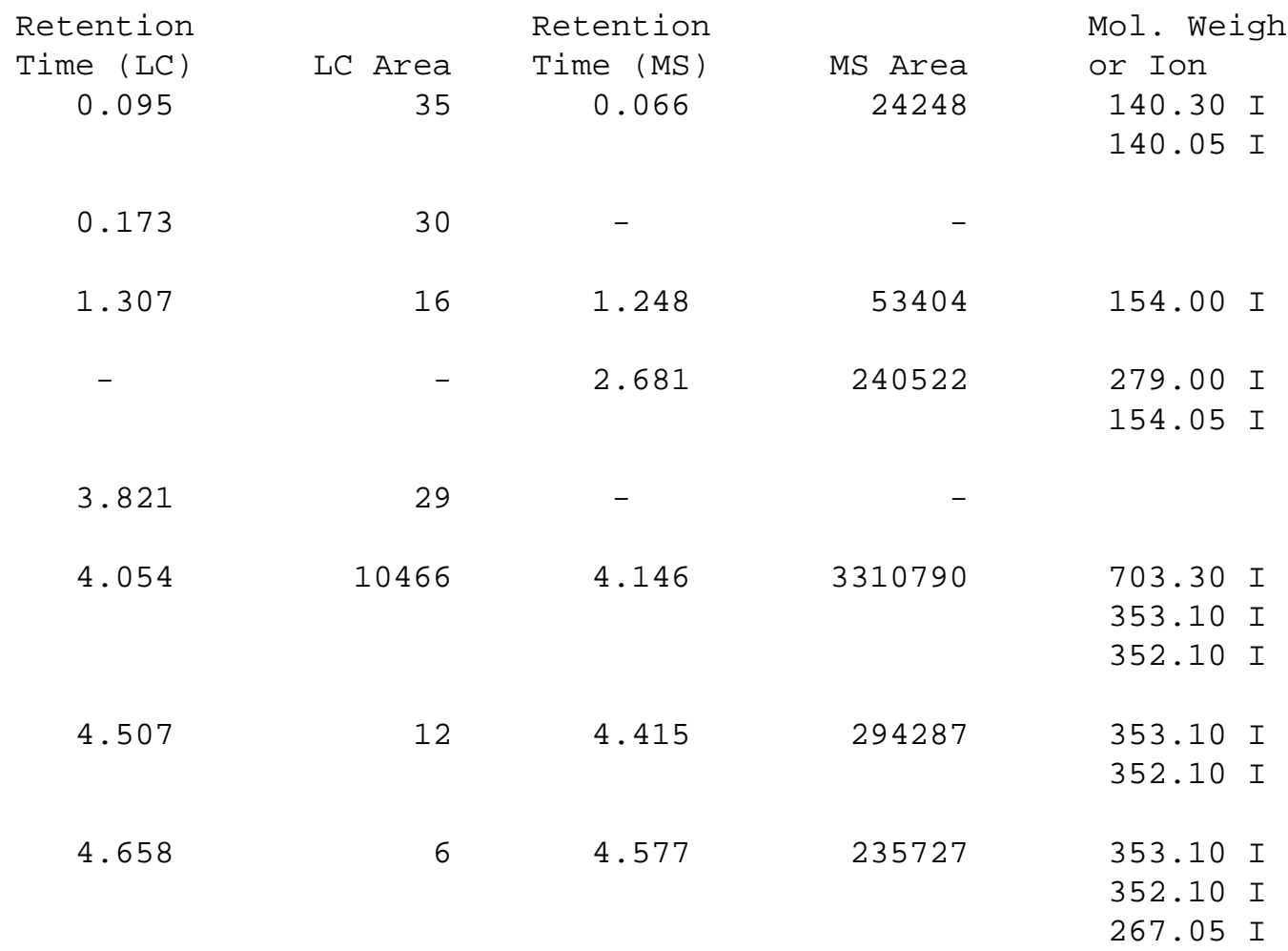

5.121

22

5.524

6
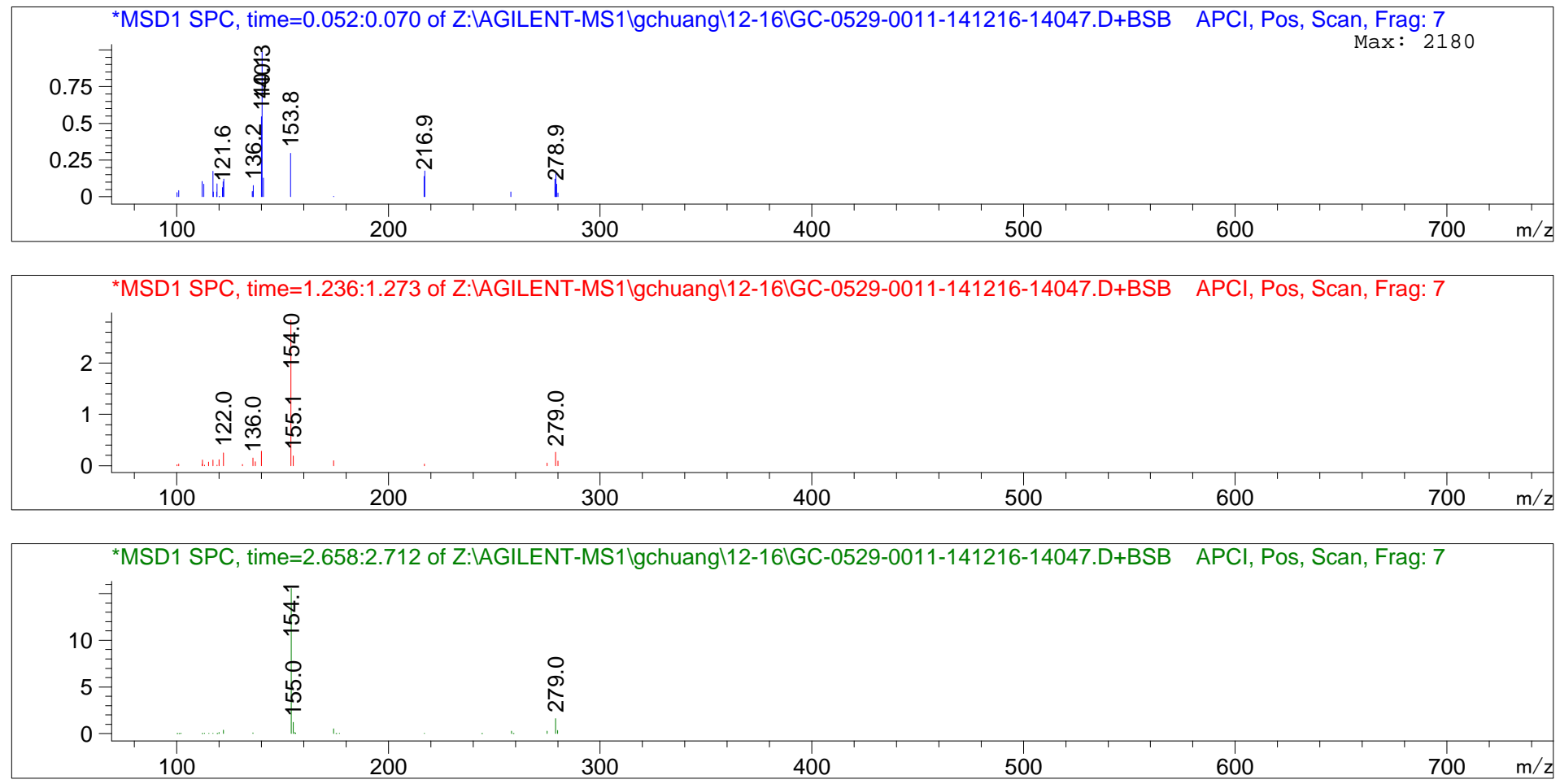
Data File Z: \AGILENT-MS1\GCHUANG \12-16\GC-0529-0011-141216-14047.D

Sample Name: gc-0529-001
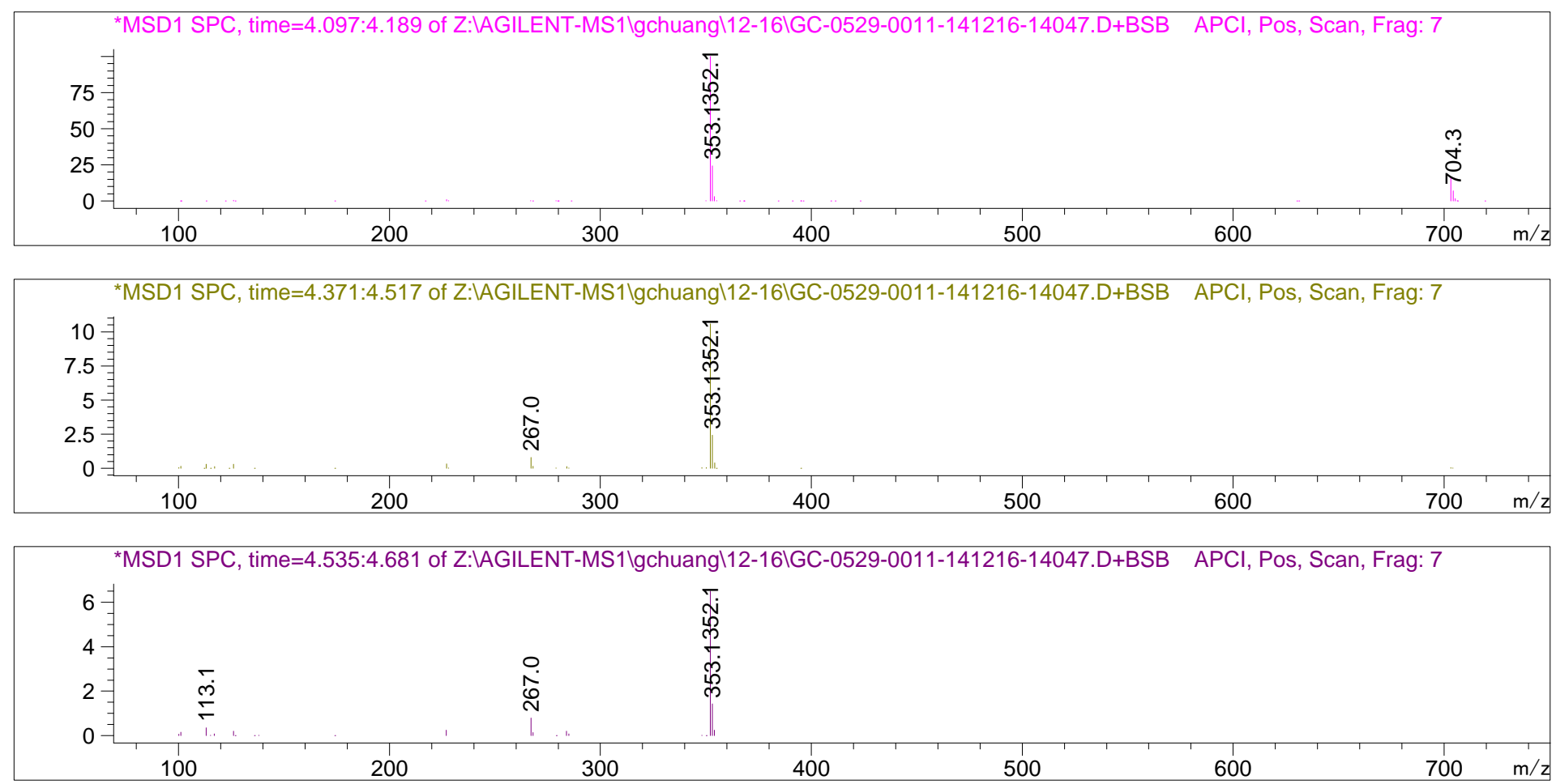


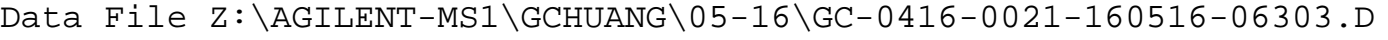

Sample Name: gc-0416-002

Easy-Access Method: '10M_POS'

\begin{tabular}{|c|c|c|c|}
\hline $\begin{array}{l}\text { Acq. Operator } \\
\text { Sample Operator }\end{array}$ & $\begin{array}{l}: \text { chemequip } \\
: \text { chemequip }\end{array}$ & & \\
\hline Acq. Instrument & : AGILENTMS1 & Location & : Vial 67 \\
\hline Injection Date & : 5/16/2016 2:32:04 PM & $\begin{array}{r}\operatorname{Inj} \\
j \text { Volume }\end{array}$ & $\begin{array}{ccc}: & 1 \\
: & 5.000 \mu 1\end{array}$ \\
\hline Method & : C $: \backslash \mathrm{CHEM} 32 \backslash 2 \backslash \mathrm{METHODS} \backslash 10 \mathrm{M} \_\mathrm{POS} . \mathrm{M}$ & & \\
\hline Last changed & $\begin{aligned}: & 5 / 16 / 2016 \text { 2:31:01 PM by chemequi } \\
& \text { (modified after loading) }\end{aligned}$ & & \\
\hline Sample Info & : Easy-Access Method: '10M_POS' & & \\
\hline
\end{tabular}
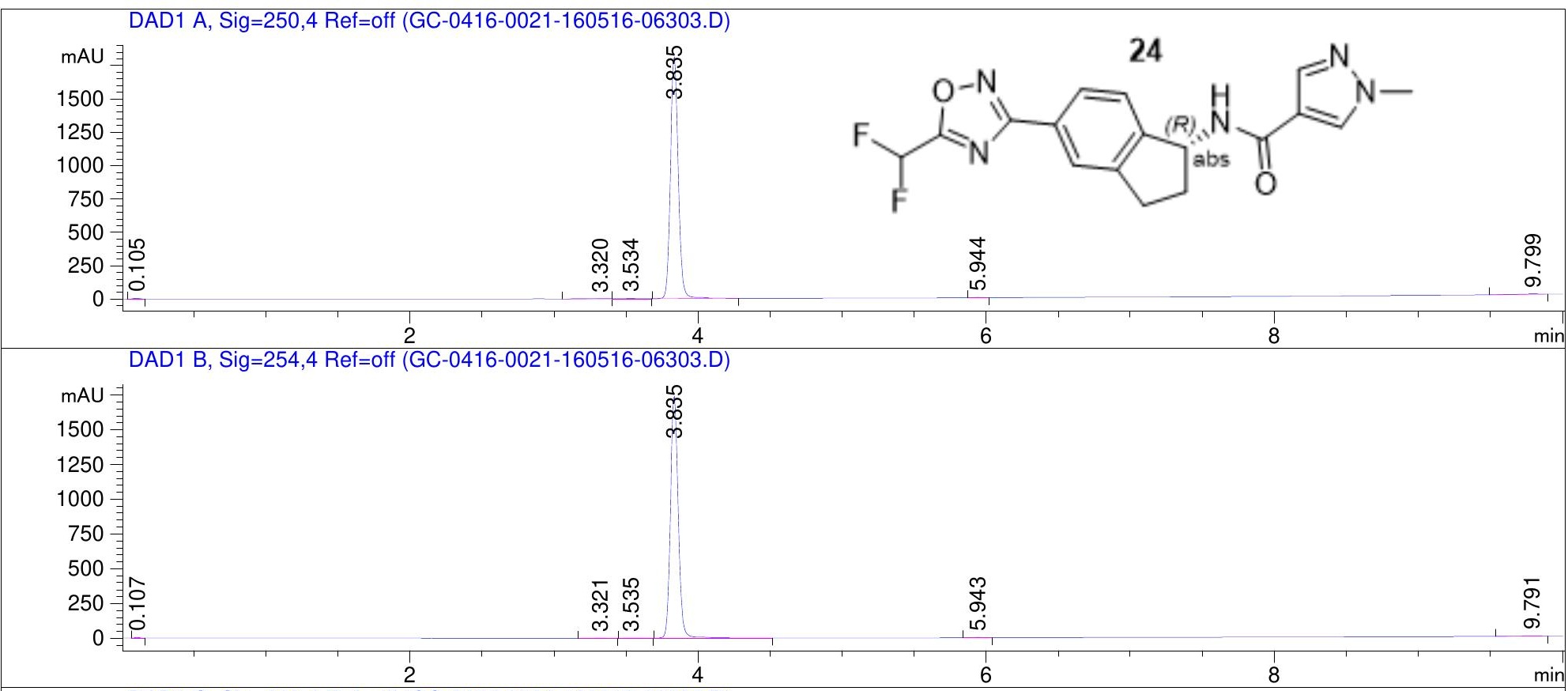

DAD1 C, Sig=210,4 Ref=off (GC-0416-0021-160516-06303.D)
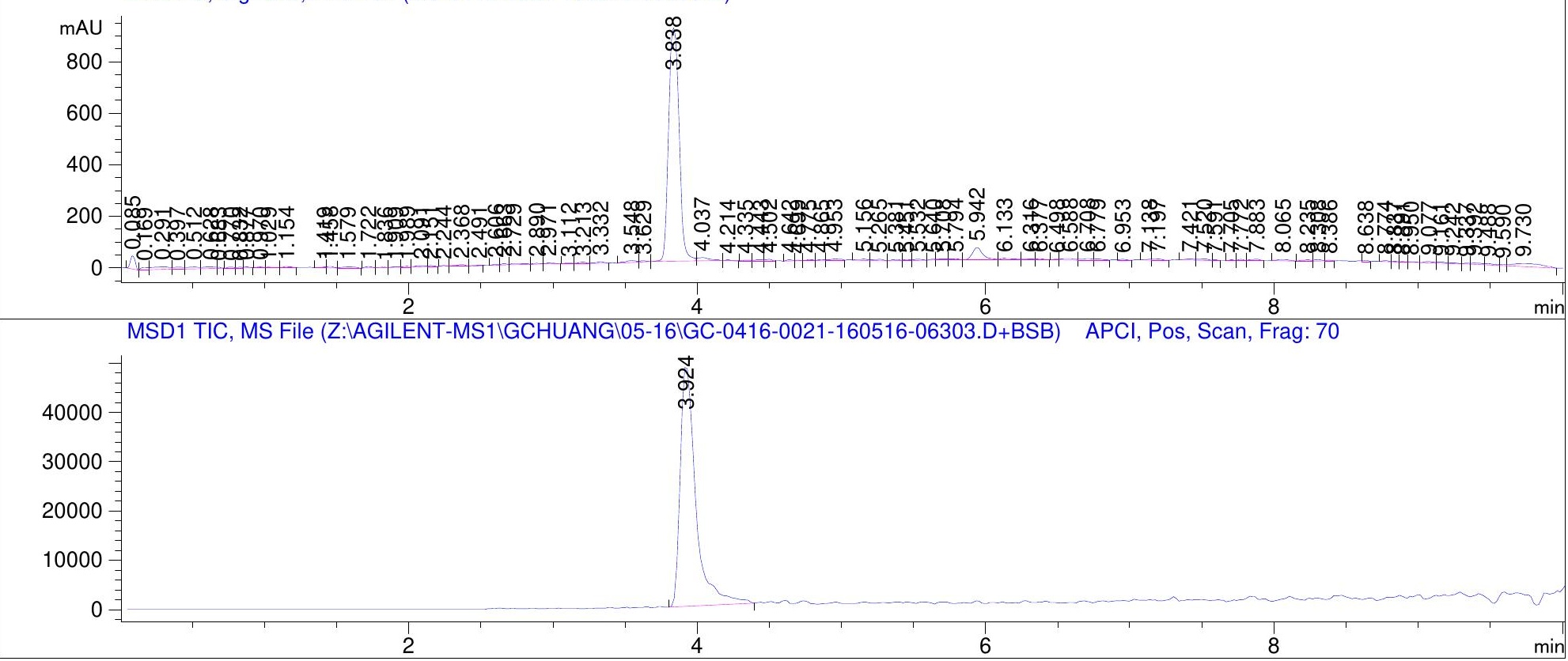
Data File Z: \AGILENT-MS1\GCHUANG \05-16\GC-0416-0021-160516-06303.D

Sample Name: gc-0416-002

MS Signal: MSD1 TIC, MS File, APCI, Pos, Scan, Frag: 70

Subtracted background from 0.000 to $3.000 \mathrm{~min}$.

Spectra averaged over upper half of peaks.

Noise Cutoff: 1000 counts.

Reportable Ion Abundance: > 10\%.

LC Signal: DAD1 A, Sig=250,4 Ref=off

Peak matching window: $0.1 \mathrm{~min}$

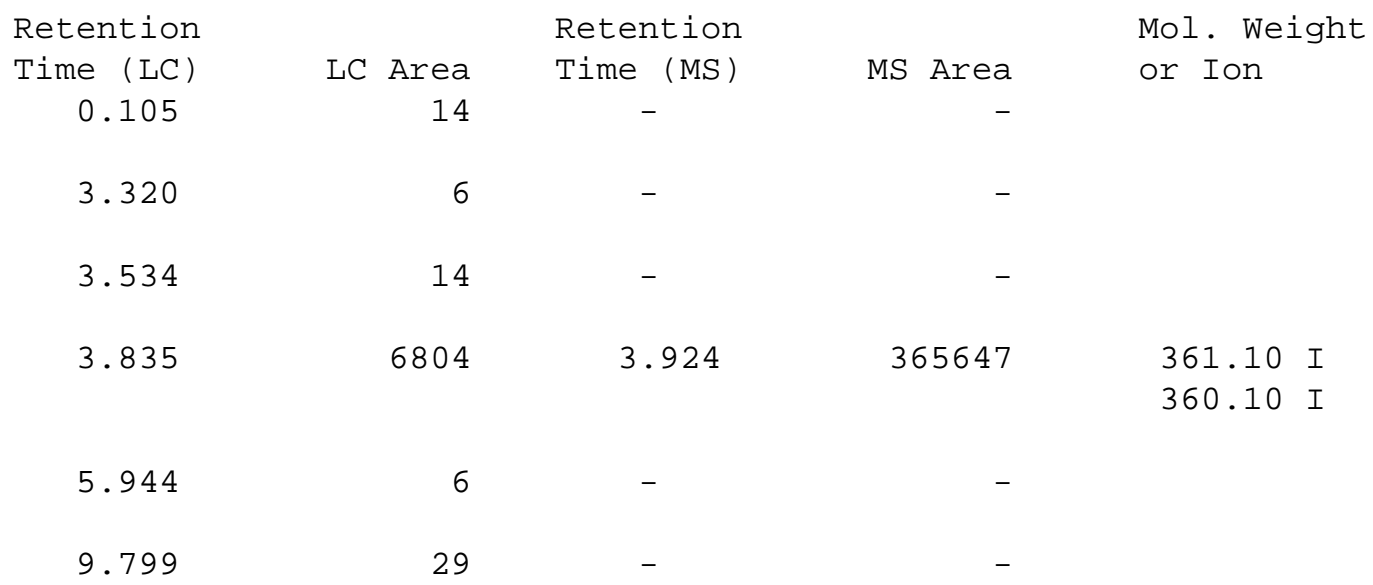

*MSD1 SPC, time=3.897:3.970 of Z:AAGILENT-MS1।gchuangl05-16|GC-0416-0021-160516-06303.D+BSB APCI, Pos, Scan, Frag: 7

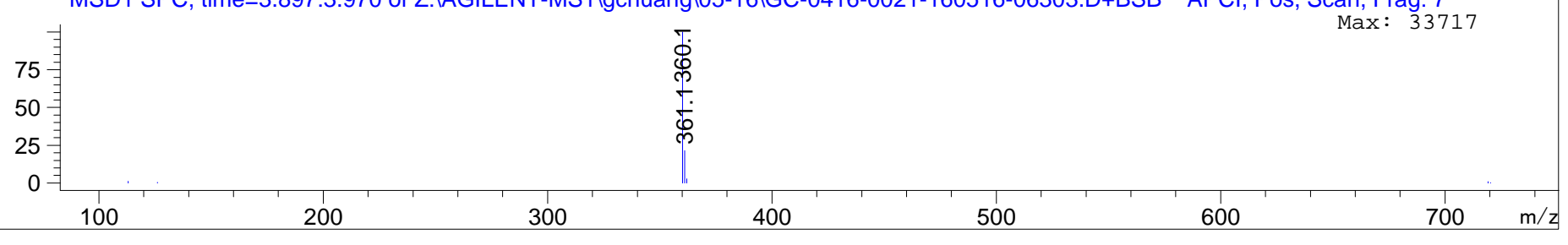




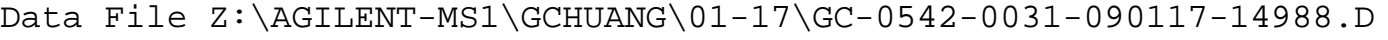

Sample Name: gc-0542-003

Easy-Access Method: '10M_POS'

$=========================$
Acq. Operator : chemequip

Sample Operator : chemequip

Acq. Instrument : AGILENTMS1

Injection Date : 1/9/2017 1:59:11 PM

Location : Vial 61

$\operatorname{Inj}: \quad 1$

Method : C : \CHEM32 $2 \backslash$ METHODS $\backslash 10$ M_POS.M

Last changed : 1/9/2017 1:58:08 PM by chemequip

(modified after loading)

Sample Info : Easy-Access Method: '10M_PoS'
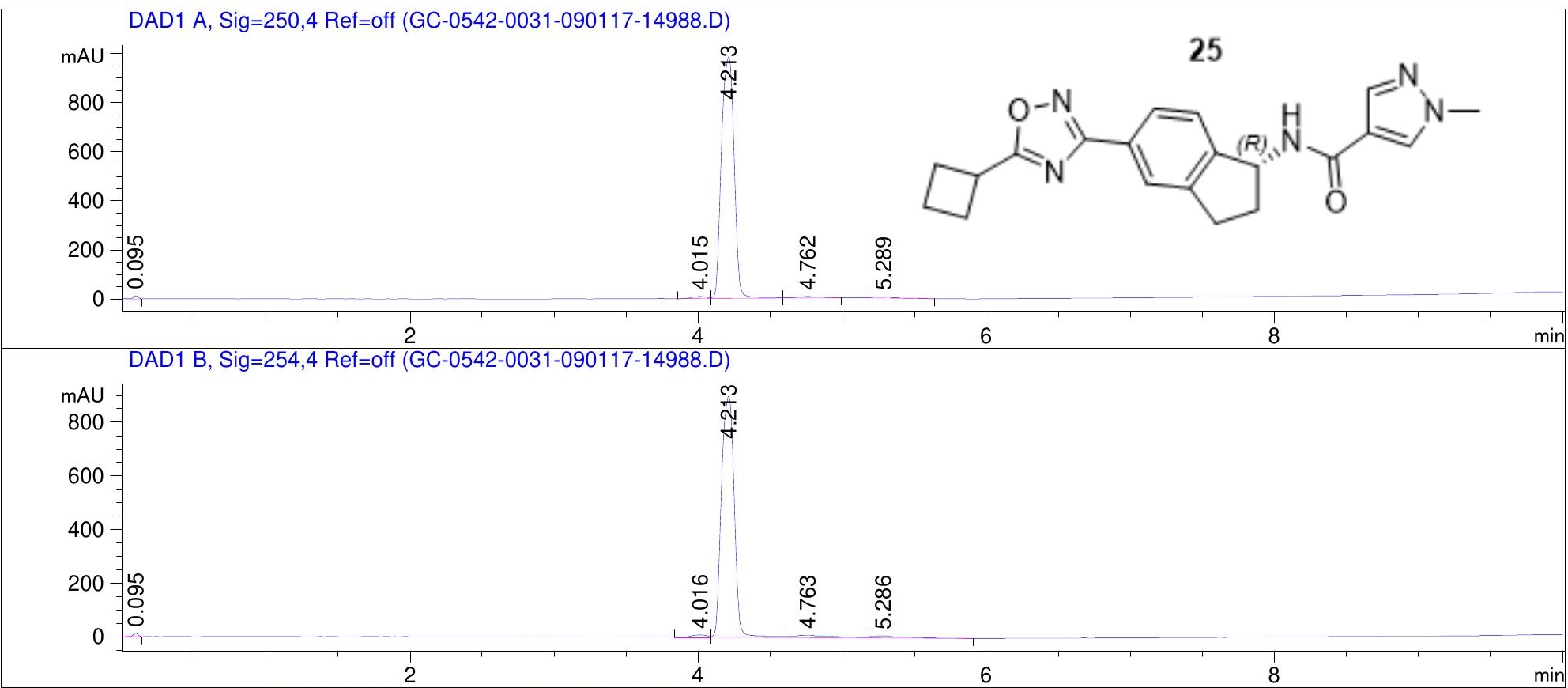

DAD1 C, Sig=210,4 Ref=off (GC-0542-0031-090117-14988.D)
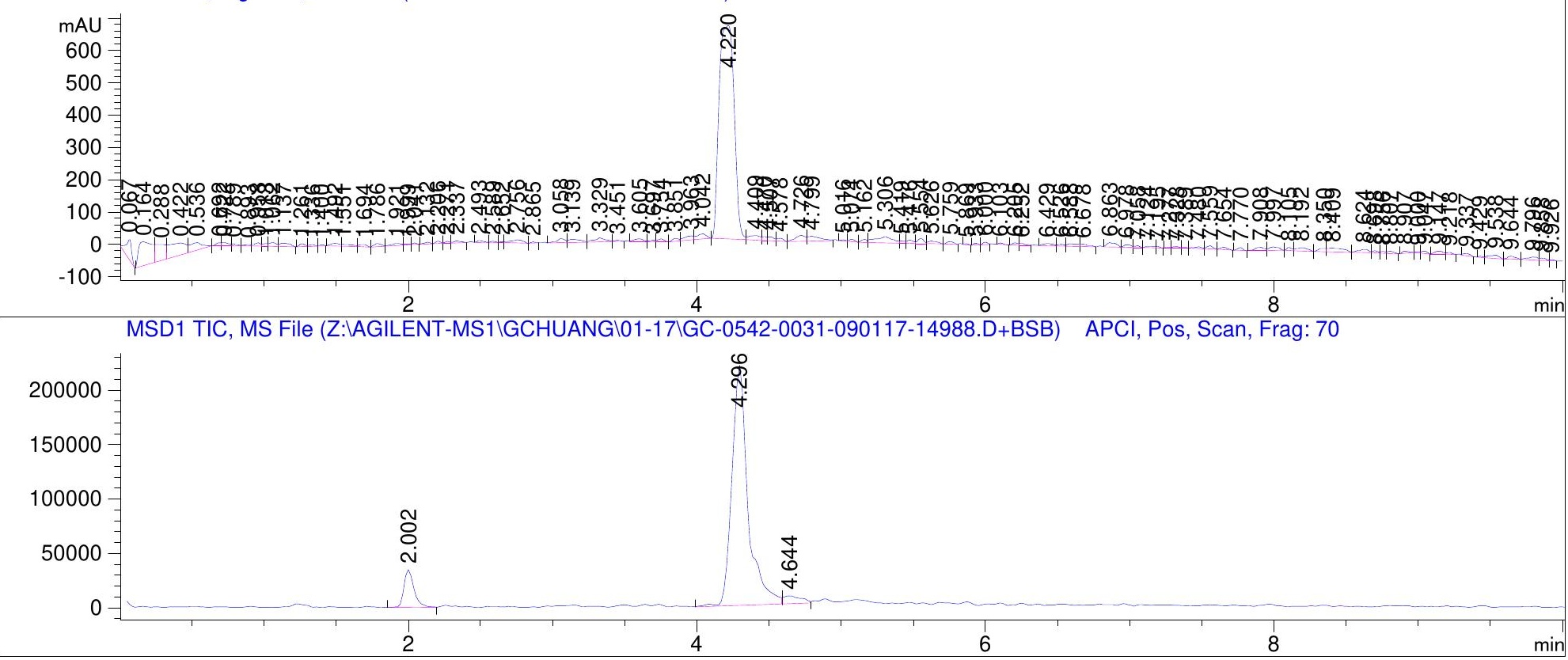


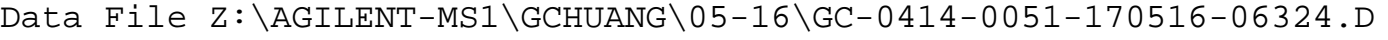

Sample Name: gc-0414-005

Easy-Access Method: '10M_POS'

$========================$
Acq. Operator : chemequip

Sample Operator : chemequip

Acq. Instrument : AGILENTMS1

Injection Date : 5/17/2016 8:46:43 AM

Location : Vial 88

Inj : 1

Method : C : \CHEM32 $2 \backslash$ METHODS $\backslash 10 M \_P O S . M$

Last changed : 5/17/2016 8:45:47 AM by chemequip

(modified after loading)

Sample Info : Easy-Access Method: '10M_Pos'
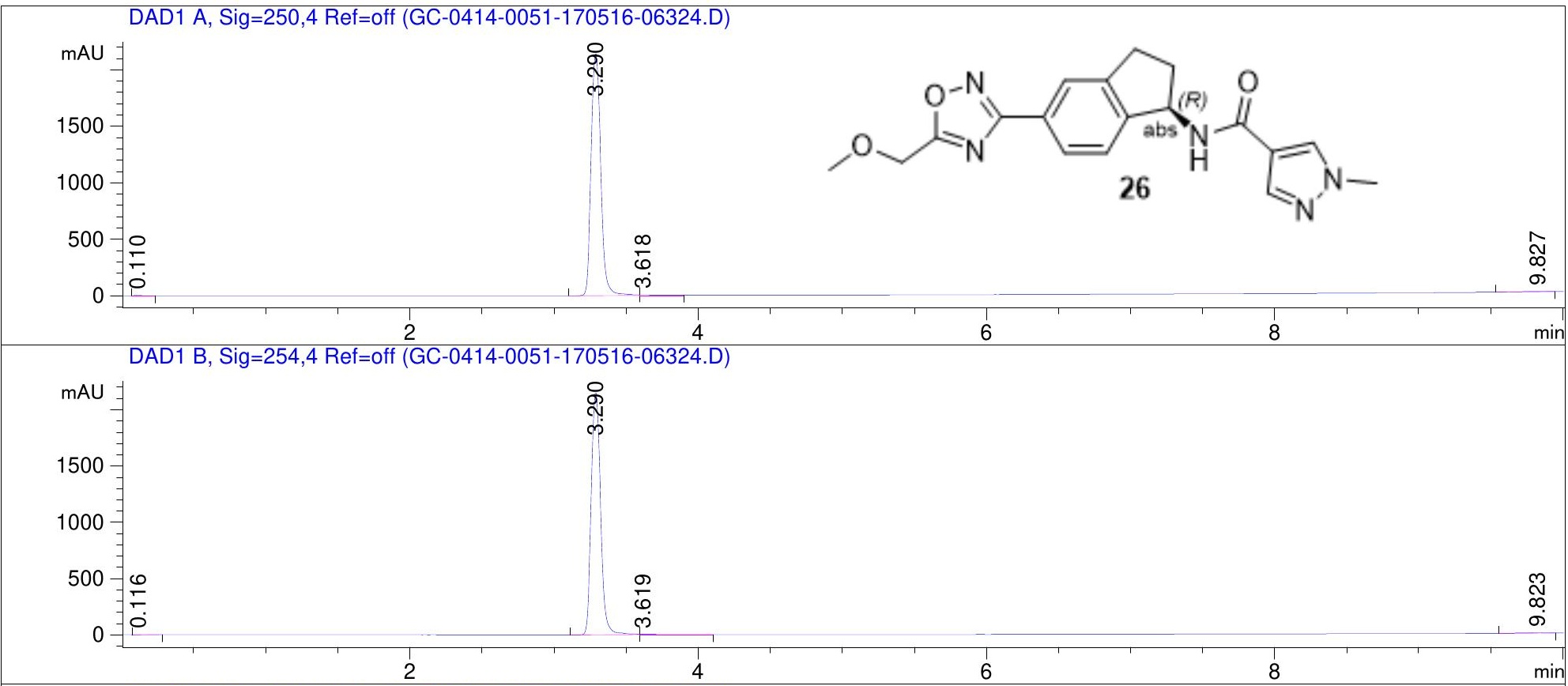

DAD1 C, Sig=210,4 Ref=off (GC-0414-0051-170516-06324.D)

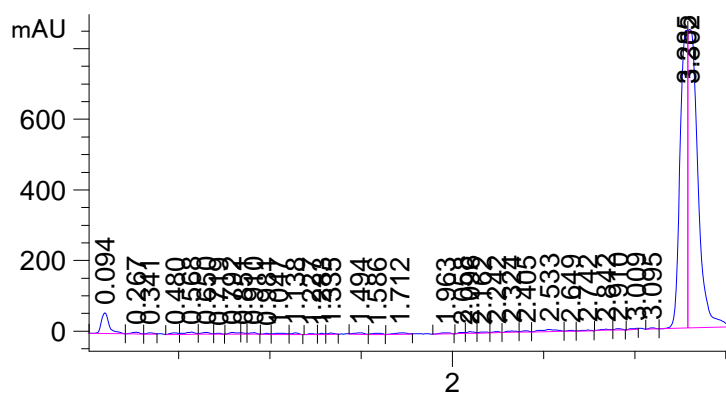

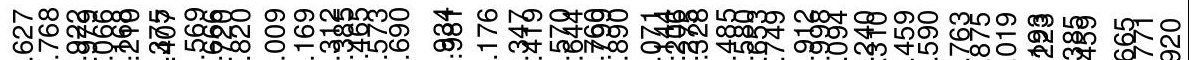

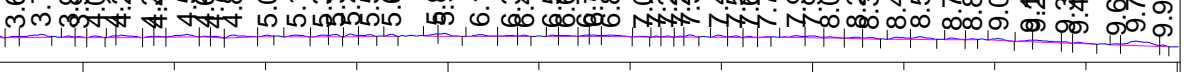

4

6

8 $\min$

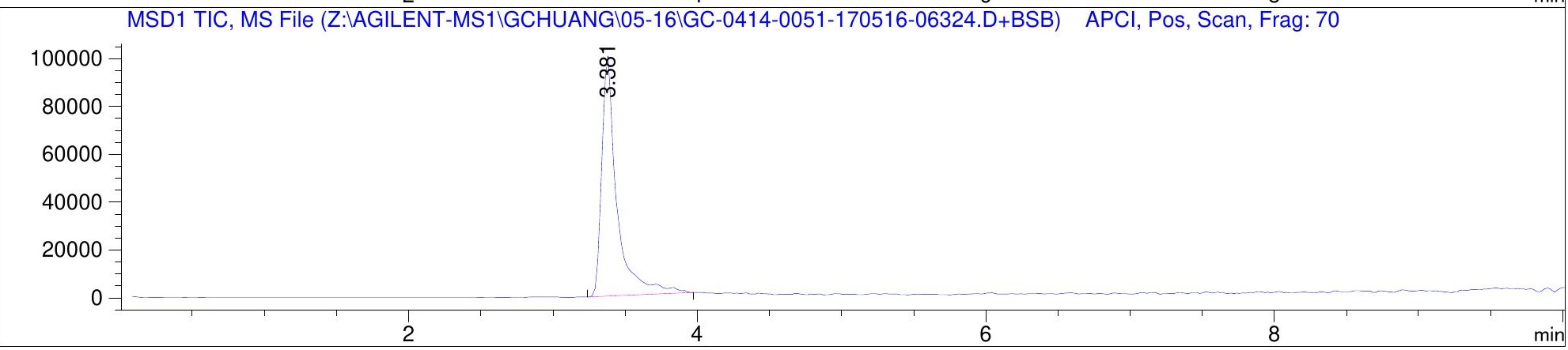




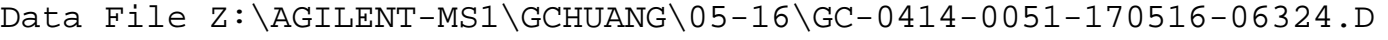

Sample Name: gc-0414-005

MS Signal: MSD1 TIC, MS File, APCI, Pos, Scan, Frag: 70

Subtracted background from 0.000 to $3.000 \mathrm{~min}$.

Spectra averaged over upper half of peaks.

Noise Cutoff: 1000 counts.

Reportable Ion Abundance: > 10\%.

LC Signal: DAD1 A, Sig=250,4 Ref=off

Peak matching window: $0.1 \mathrm{~min}$

$\begin{array}{crrrr}\begin{array}{c}\text { Retention } \\ \text { Time (LC) } \\ 0.110\end{array} & \text { LC Area } & \begin{array}{r}\text { Retention } \\ \text { Time (MS) }\end{array} & \text { MS Area } & \text { Mol. Weight } \\ 3.290 & 9939 & 3.381 & 734138 & 355.10 \text { I } \\ & & - & & 354.10 \text { I } \\ 3.618 & 48 & - & -\end{array}$

${ }^{*}$ MSD1 SPC, time=3.347:3.420 of Z:IAGILENT-MS1/gchuangl05-16/GC-0414-0051-170516-06324.D+BSB APCI, Pos, Scan, Frag: 7

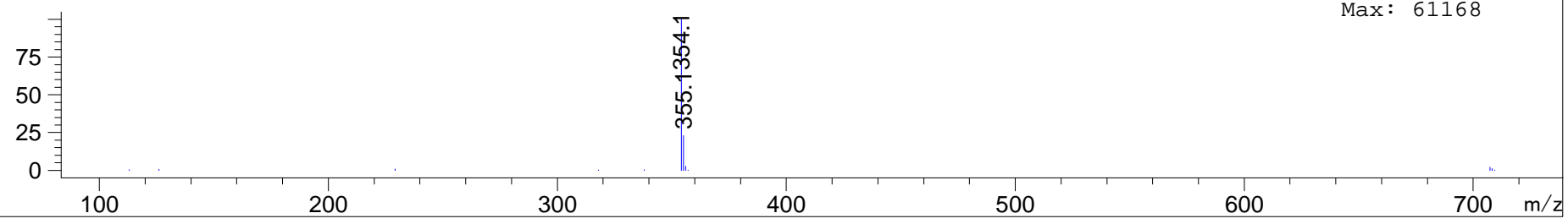


Data File D: \MassHunter\gchuang \2017-10-30\10M_POS_gC-0763-001_8846.D

Sample Name: gc-0763-001

Walkup method: '10MIN POS'

\begin{tabular}{|c|c|c|c|}
\hline $\begin{array}{l}\text { Acq. Operator } \\
\text { Sample Operator }\end{array}$ & $\begin{array}{l}: \text { chemequip } \\
: \text { Grace }\end{array}$ & & \\
\hline Acq. Instrument & : LCMS & Location & $\mathrm{P} 1-\mathrm{D} 1$ \\
\hline Injection Date & $: 10 / 30 / 2017 \quad 10: 27: 07 \mathrm{AM}$ & $\operatorname{Inj}$ & $: \quad 1$ \\
\hline Method & $: C: \backslash$ Chem32\1\Methods \10M_POS.M & In] volume & \\
\hline Last changed & : 2/6/2017 3:06:20 PM by SYSTEM & & \\
\hline Sample Info & : Walkup method: '10MIN POS' & & \\
\hline
\end{tabular}

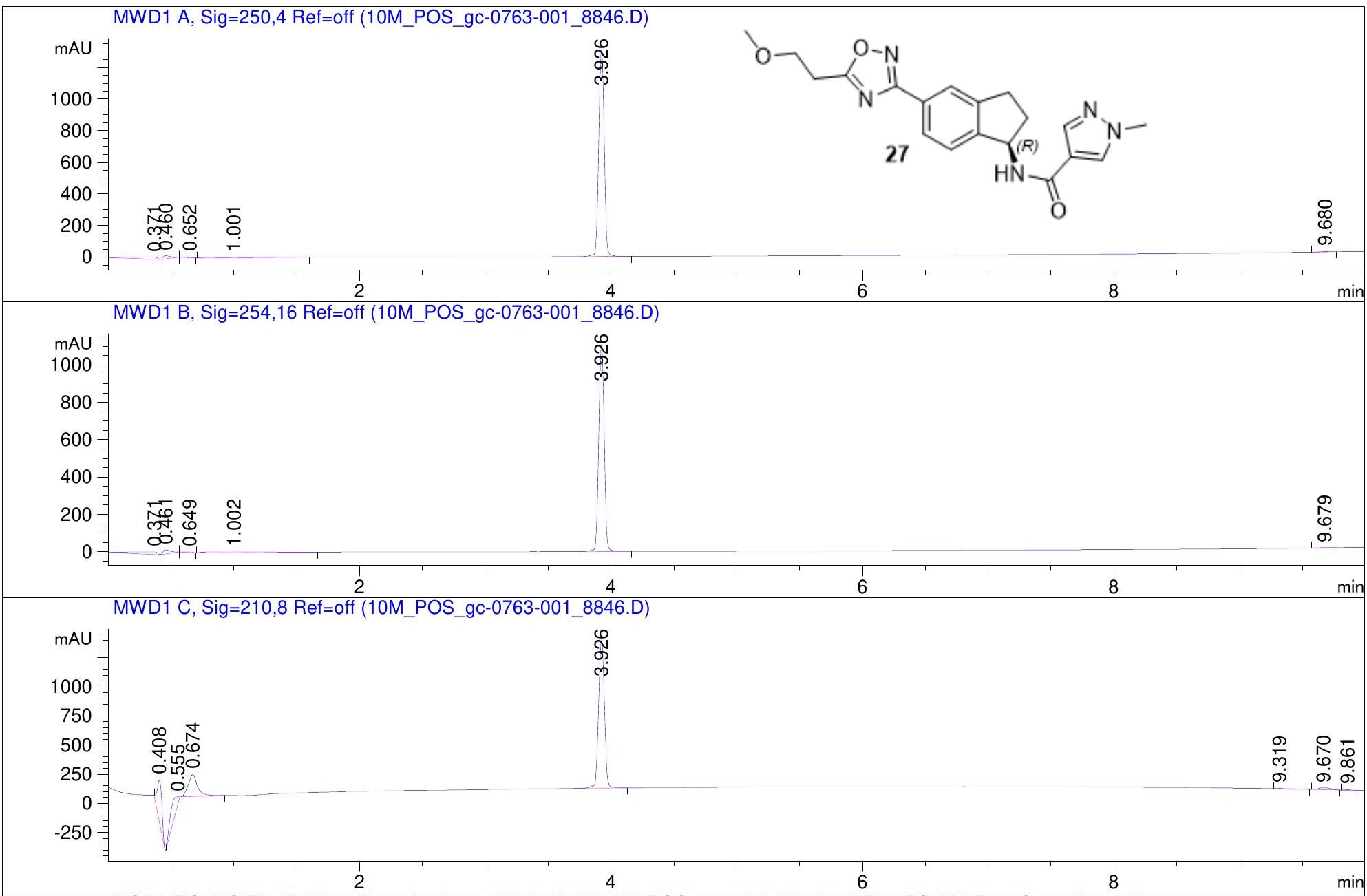

MSD1 TIC, MS File (D:MassHunterlgchuangl2017-10-30110M_POS_gc-0763-001_8846.D) ES-API, Pos, Scan, Frag: 70

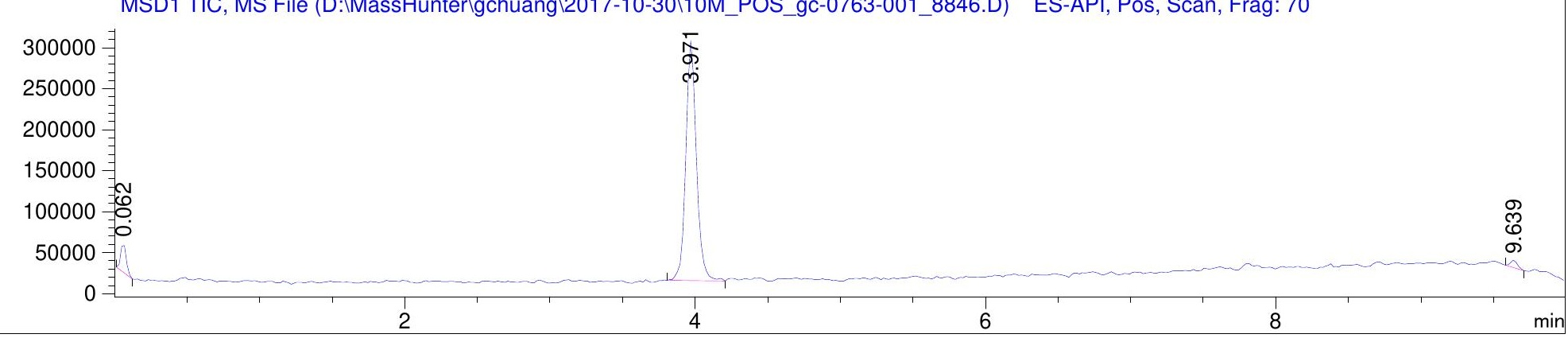


Data File D: \MassHunter\gchuang \2017-10-30\10M_POS_gC-0763-001_8846.D

Sample Name: gc-0763-001

MS Signal: MSD1 TIC, MS File, ES-API, Pos, Scan, Frag: 70

Spectra averaged over upper half of peaks.

Noise Cutoff: 1000 counts.

Reportable Ion Abundance: > 10\%.

Retention

Time (MS)

0.062

1481568

9.639

30031
Mol. Weight

or Ion

$$
\begin{aligned}
& 130.30 \mathrm{I} \\
& 130.10 \mathrm{I} \\
& 129.90 \mathrm{I} \\
& 128.30 \mathrm{I} \\
& 123.00 \mathrm{I} \\
& 111.20 \mathrm{I}
\end{aligned}
$$

$736.30 \mathrm{I}$

$735.30 \mathrm{I}$

$369.20 \mathrm{I}$

$368.15 \mathrm{I}$

$146.95 \mathrm{I}$

$145.00 \mathrm{I}$

$133.95 \mathrm{I}$

$132.00 \mathrm{I}$

*MSD1 SPC, time=0.052:0.071 of D:MassHunterlgchuangl2017-10-30\10M_POS_gc-0763-001_8846.D ES-API, Pos, Scan, Frag:
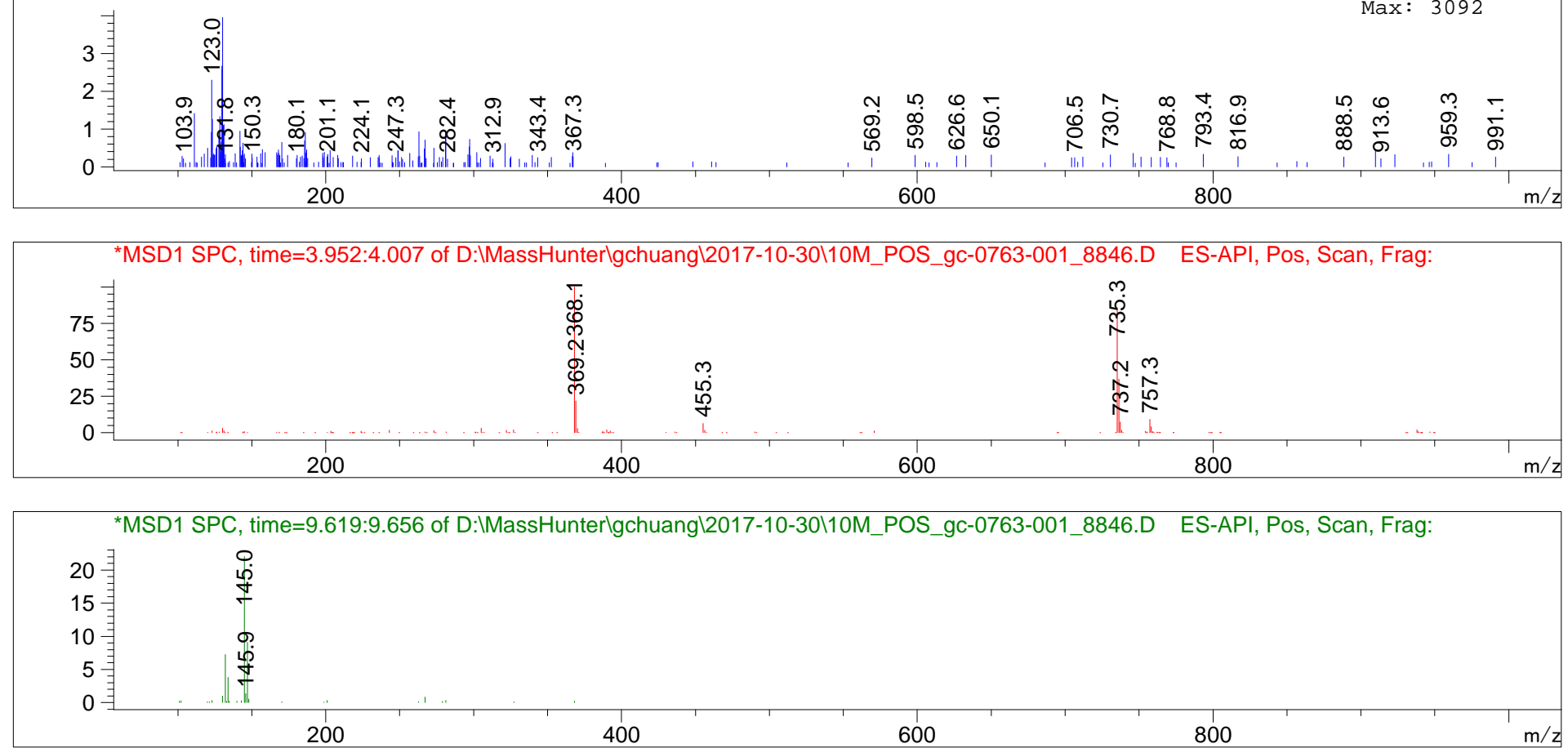
Data File D: \MassHunter\gchuang \2017-10-31\10M_POS_gc-0764-005_8758.D

Sample Name: gc-0764-005

Walkup method: '10MIN POS'
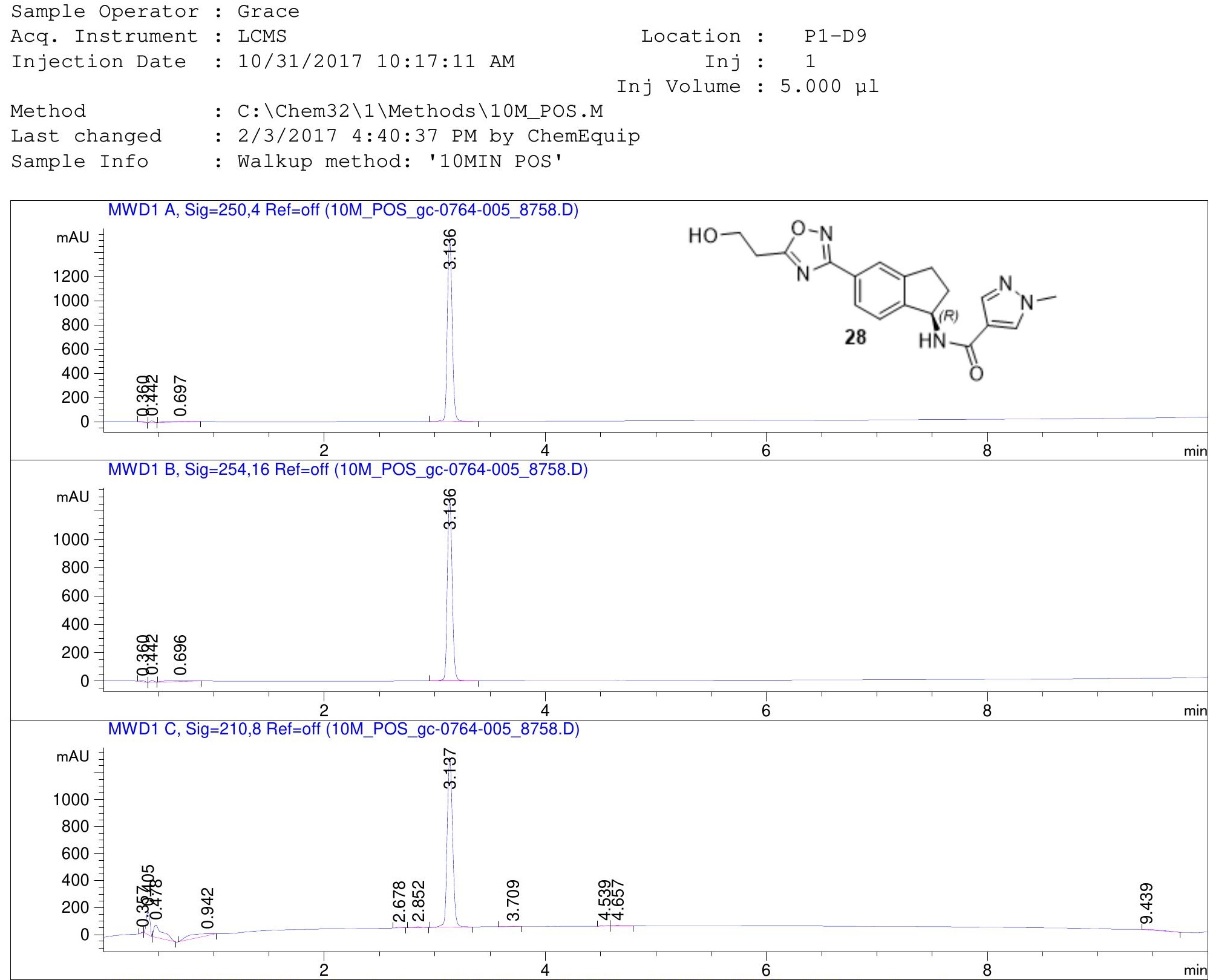

MSD1 TIC, MS File (D:IMassHunterlgchuangl2017-10-31110M_POS_gc-0764-005_8758.D) APCI, Pos, Scan, Frag: 70

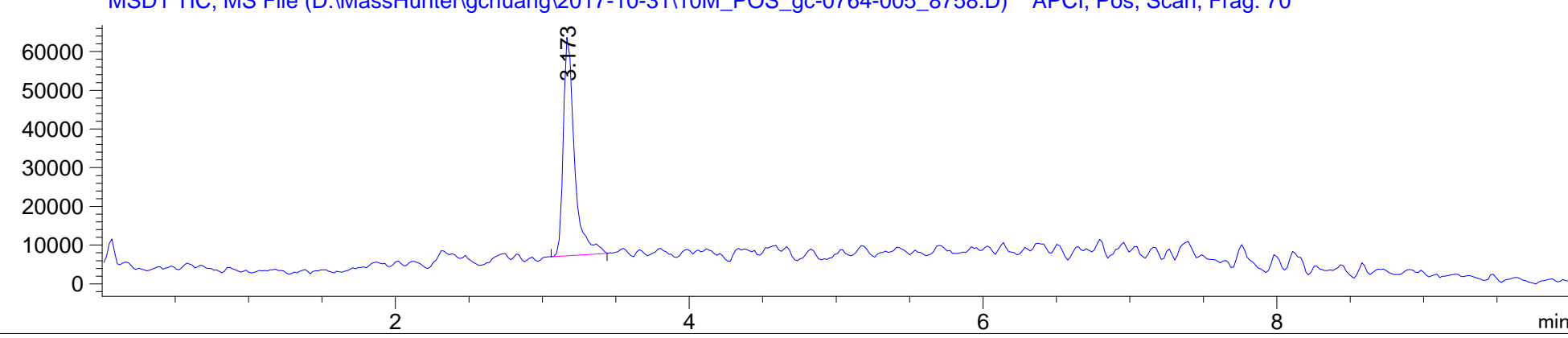


Data File D: \MassHunter\gchuang \2017-10-31\10M_POS_gC-0764-005_8758.D

Sample Name: gc-0764-005

MS Signal: MSD1 TIC, MS File, APCI, Pos, Scan, Frag: 70 Spectra averaged over upper half of peaks.

Noise Cutoff: 1000 counts.

Reportable Ion Abundance: > 10\%.

Retention

Time (MS)

3.173
MS Area

297607
Mol. Weight

or Ion

$355.10 \mathrm{I}$ $354.10 \mathrm{I}$

*MSD1 SPC, time=3.150:3.205 of D:IMassHunterlgchuangl2017-10-31110M_POS_gc-0764-005_8758.D APCI, Pos, Scan, Frag: 70

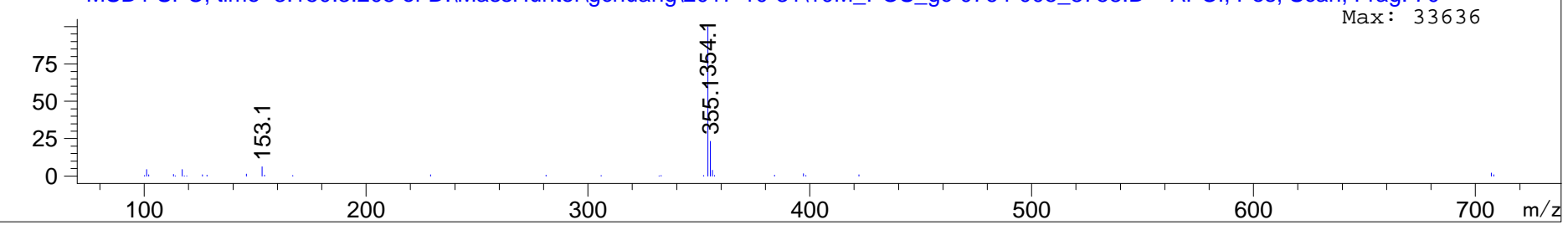




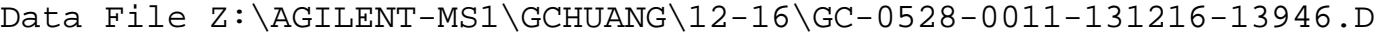

Sample Name: gc-0528-001

Easy-Access Method: '10M_POS'

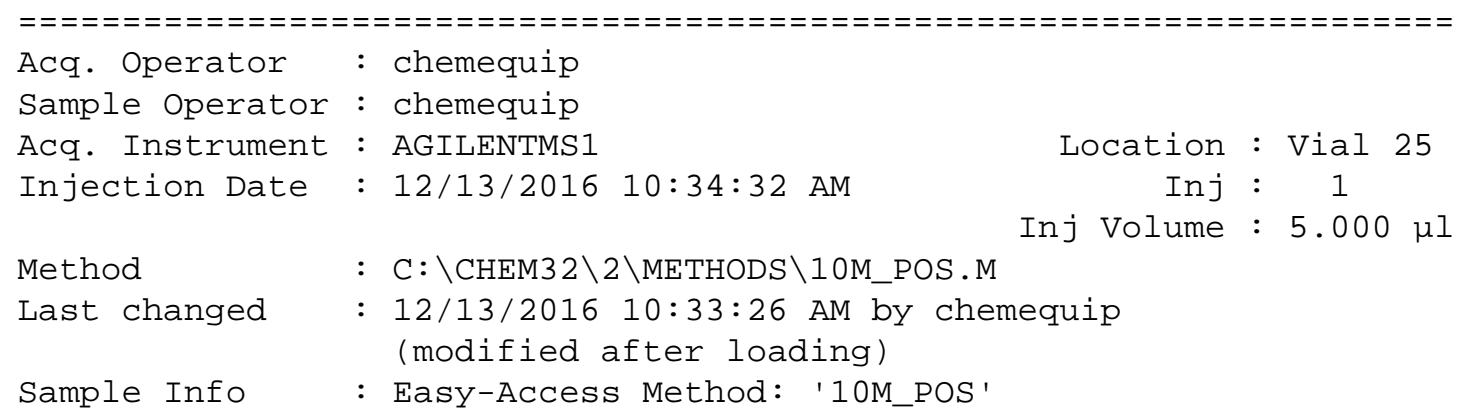
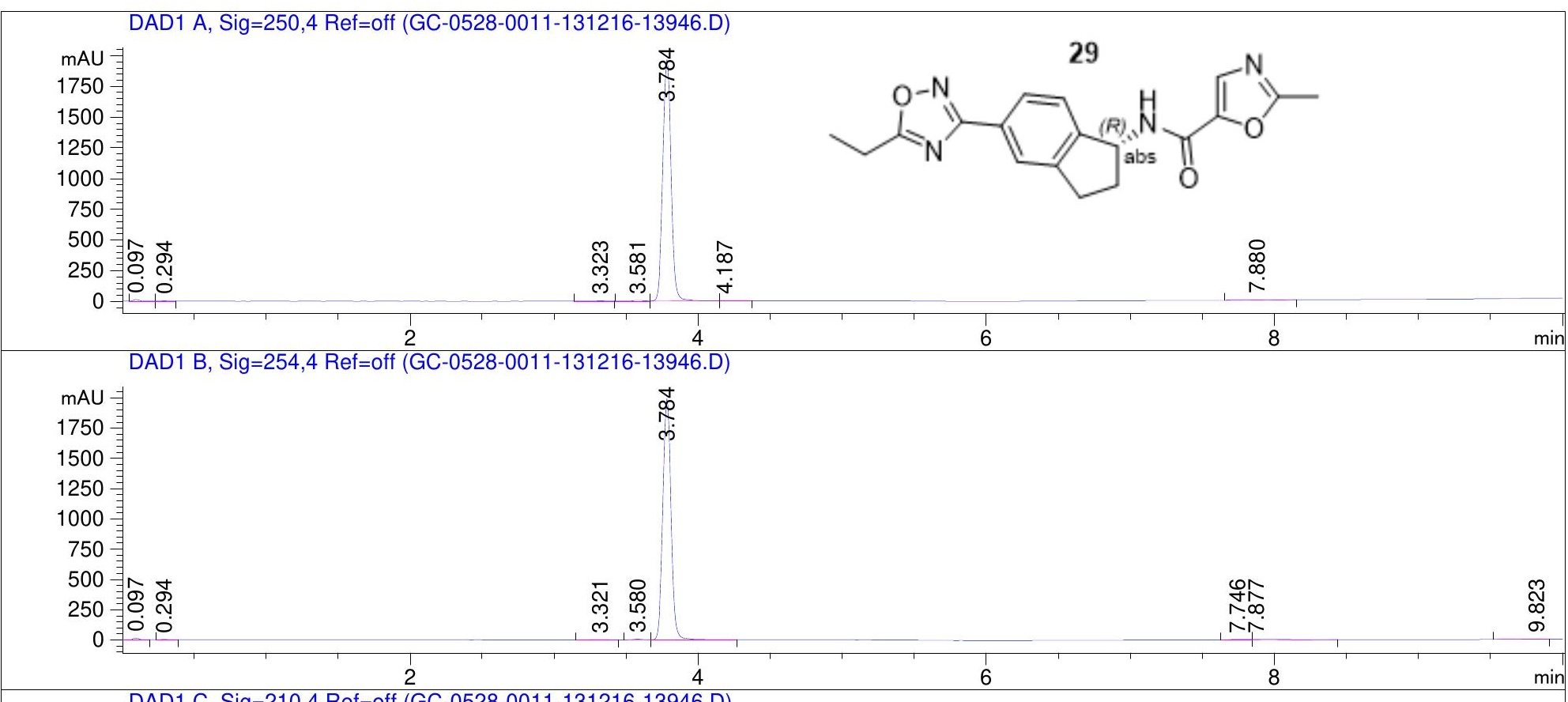

DAD1 C, Sig=210,4 Ref=off (GC-0528-0011-131216-13946.D)

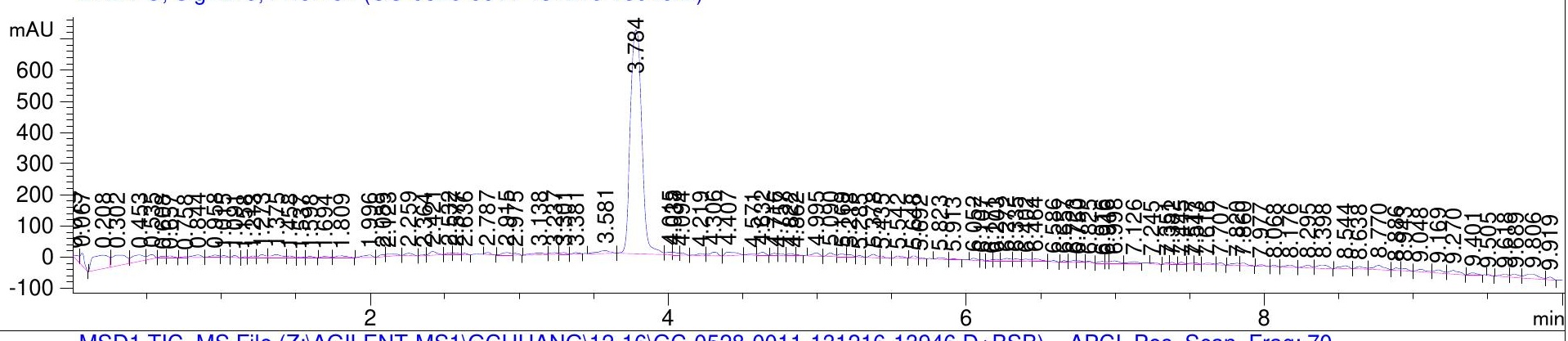
MSD1 TIC, MS File (Z:IAGILENT-MS1|GCHUANG|12-16|GC-0528-0011-131216-13946.D+BSB) APCI, Pos, Scan, Frag: 70

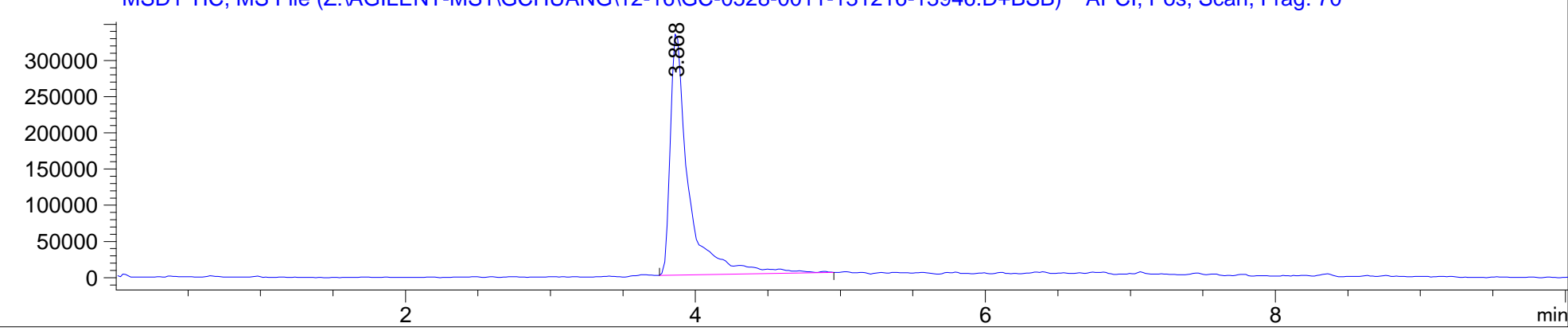




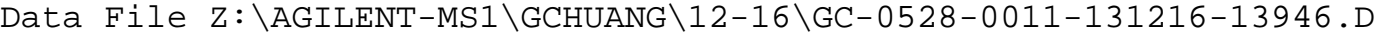

Sample Name: gc-0528-001

MS Signal: MSD1 TIC, MS File, APCI, Pos, Scan, Frag: 70

Subtracted background from 0.000 to $3.000 \mathrm{~min}$.

Spectra averaged over upper half of peaks.

Noise Cutoff: 1000 counts.

Reportable Ion Abundance: > 10\%.

LC Signal: DAD1 A, Sig=250,4 Ref=off

Peak matching window: $0.1 \mathrm{~min}$

$\begin{array}{crrrr}\begin{array}{c}\text { Retention } \\ \text { Time (LC) } \\ 0.097\end{array} & \text { LC Area } & \begin{array}{r}\text { Retention } \\ \text { Time (MS) }\end{array} & \text { MS Area } & \text { Mol. Weight } \\ 0.294 & 10 & - & - & - \\ 3.323 & 15 & - & - & \\ 3.581 & 29 & - & 2955047 & 340.10 \text { I } \\ 3.784 & 7893 & 3.868 & & 339.10 \text { I } \\ 4.187 & & & & \end{array}$

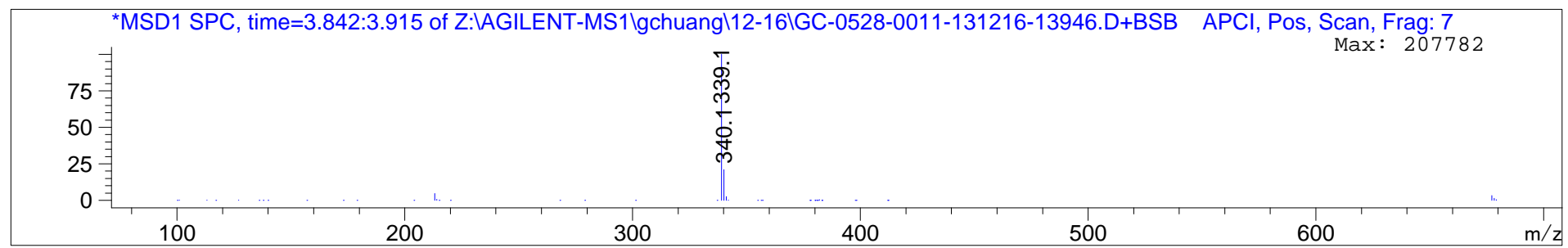




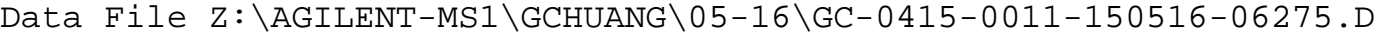

Sample Name: gc-0415-001

Easy-Access Method: '10M_POS'

$========================$
Acq. Operator : chemequip

Sample Operator : chemequip

Acq. Instrument : AGILENTMS1

Injection Date : 5/15/2016 7:15:58 AM

Location : Vial 39

Inj : 1

Method : C : \CHEM32 $2 \backslash$ METHODS $\backslash 10 M \_P O S . M$

Last changed : 5/15/2016 7:14:57 AM by chemequip

(modified after loading)

Sample Info : Easy-Access Method: '10M_Pos'
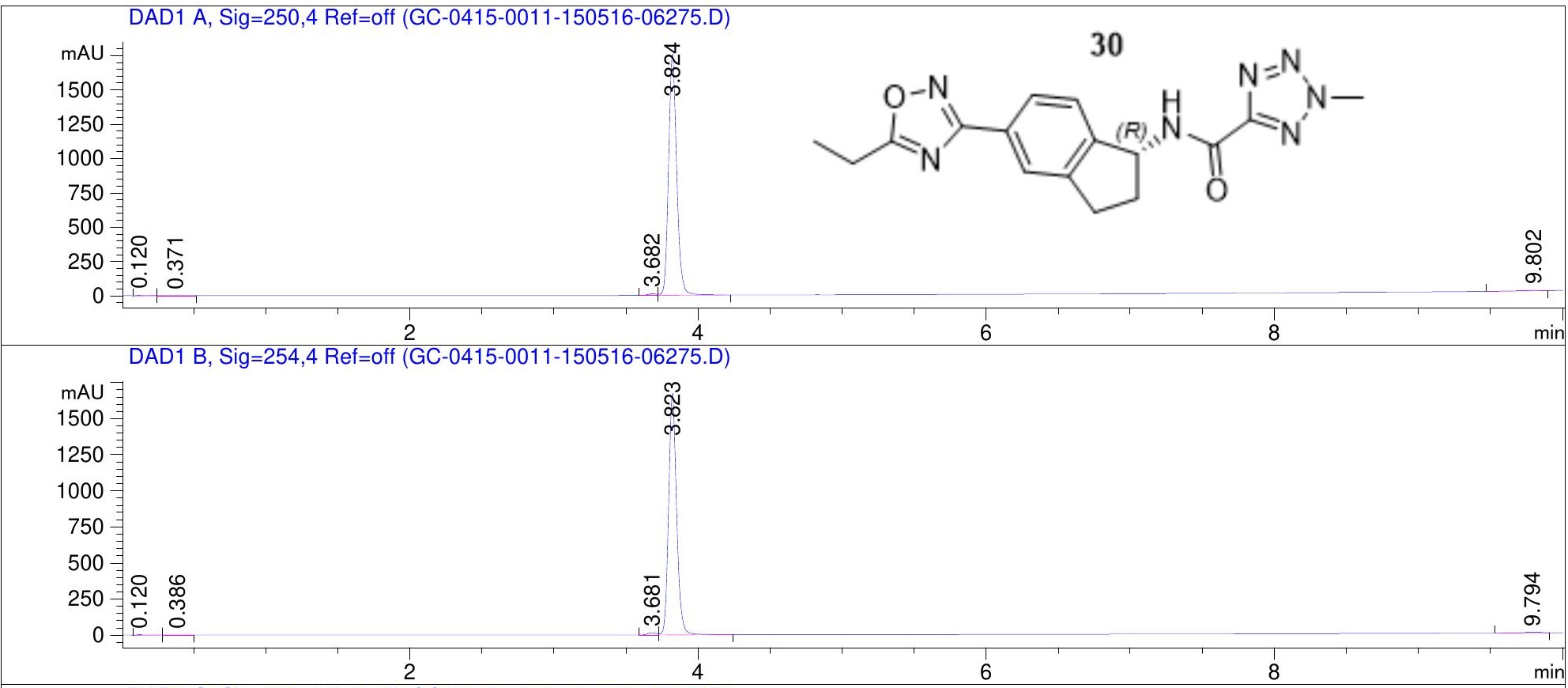

DAD1 C, Sig=210,4 Ref=off (GC-0415-0011-150516-06275.D)

Inj Volume : $5.000 \mu l$
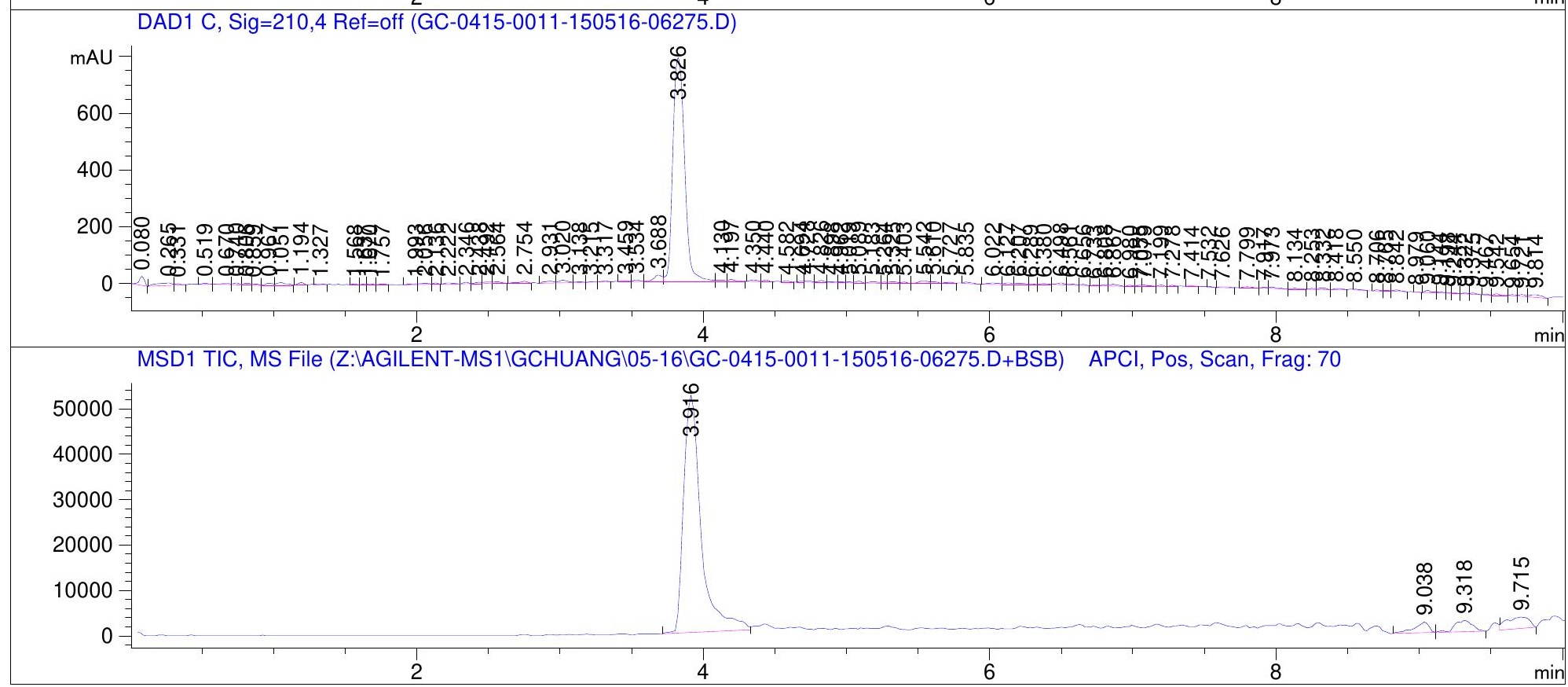


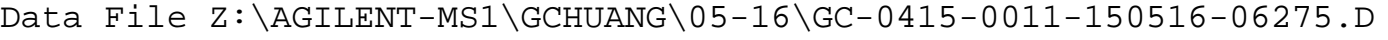

Sample Name: gc-0415-001

MS Signal: MSD1 TIC, MS File, APCI, Pos, Scan, Frag: 70

Subtracted background from 0.000 to $3.000 \mathrm{~min}$.

Spectra averaged over upper half of peaks.

Noise Cutoff: 1000 counts.

Reportable Ion Abundance: > 10\%.

LC Signal: DAD1 A, Sig=250,4 Ref=off

Peak matching window: $0.1 \mathrm{~min}$

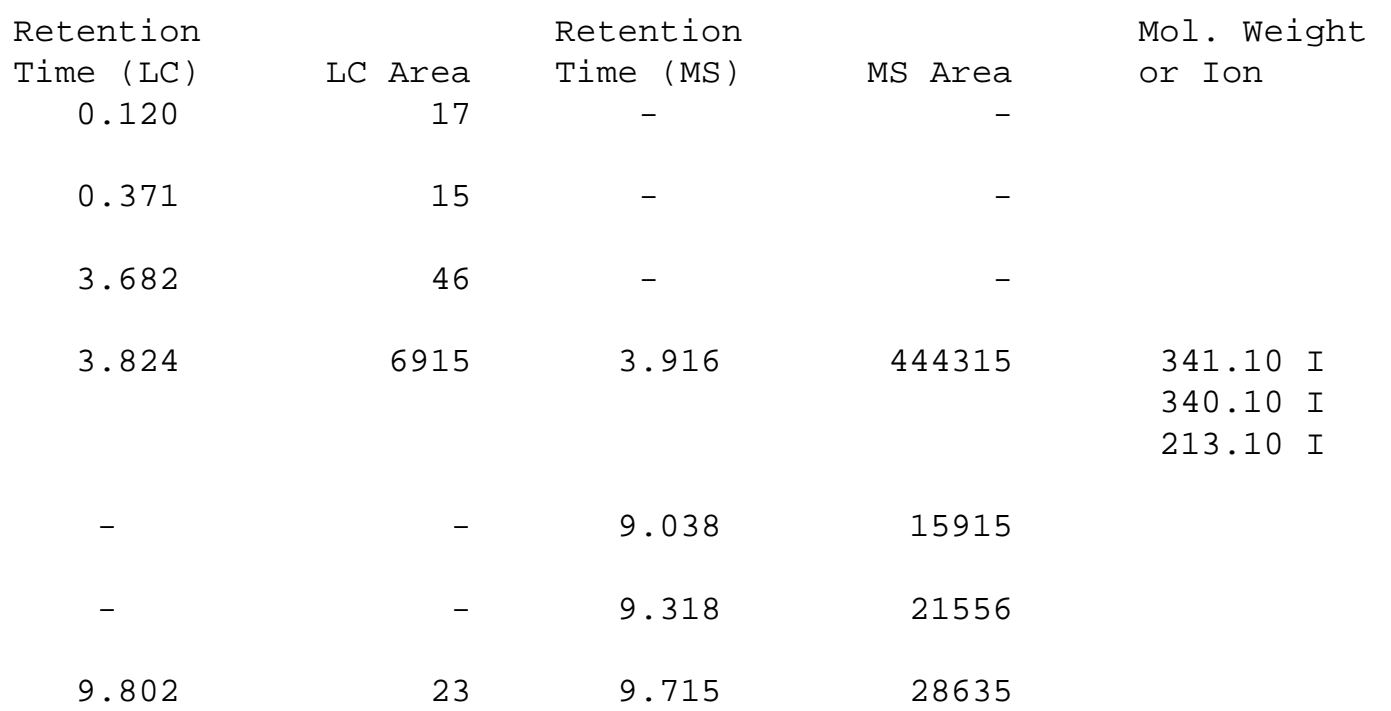
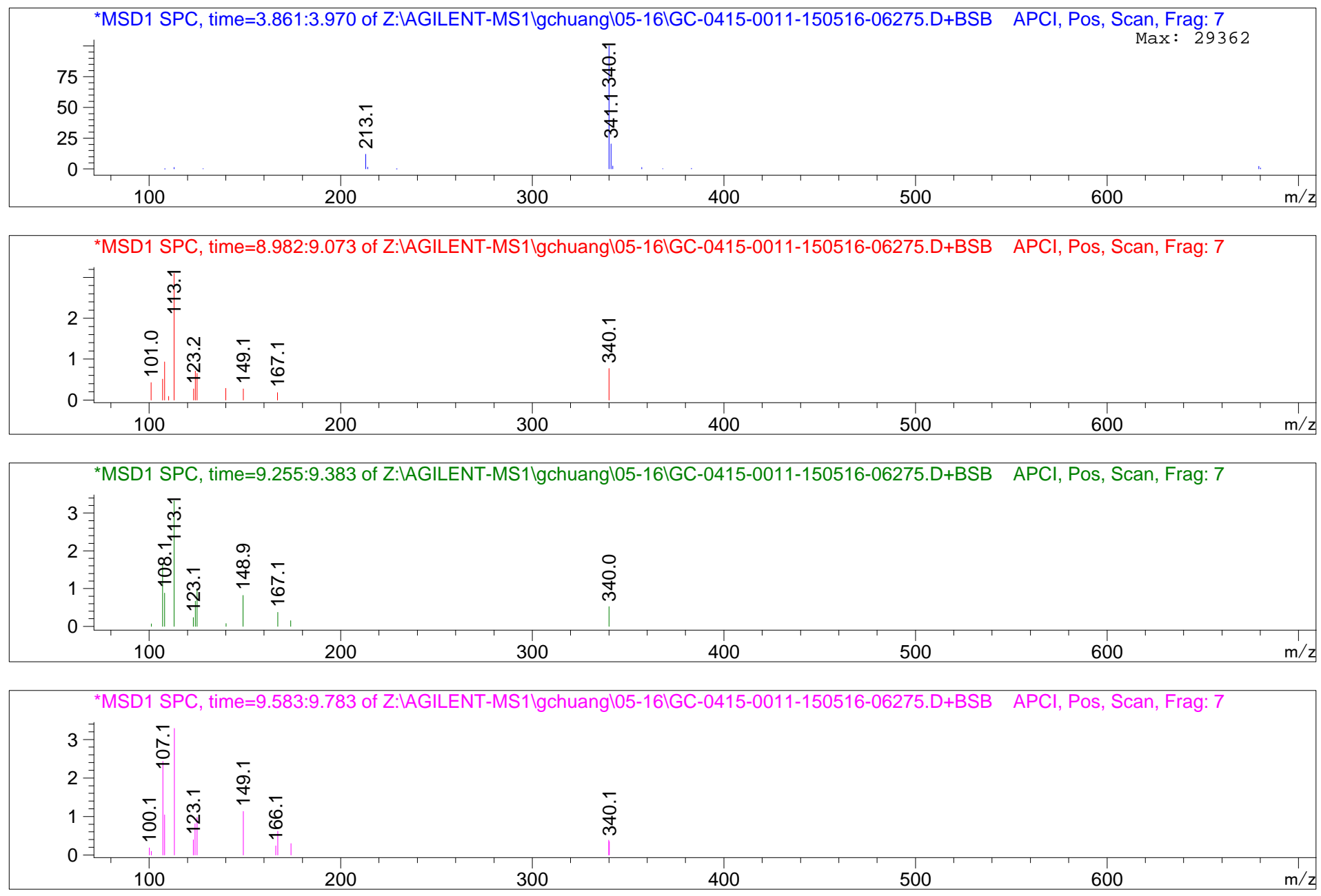


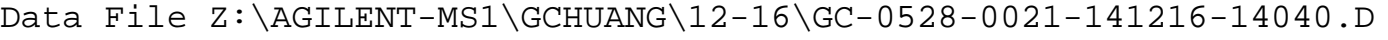

Sample Name: gc-0528-002

Easy-Access Method: '10M_POS'

$========================$
Acq. Operator : chemequip

Sample Operator : chemequip

Acq. Instrument : AGILENTMS1

Injection Date : 12/14/2016 1:29:00 PM

Location : Vial 24

Inj : 1

Method : C: \CHEM32\2\METHODS \10M_POS.M

Last changed : 12/14/2016 1:27:56 PM by chemequip

(modified after loading)

Sample Info : Easy-Access Method: '10M_Pos'
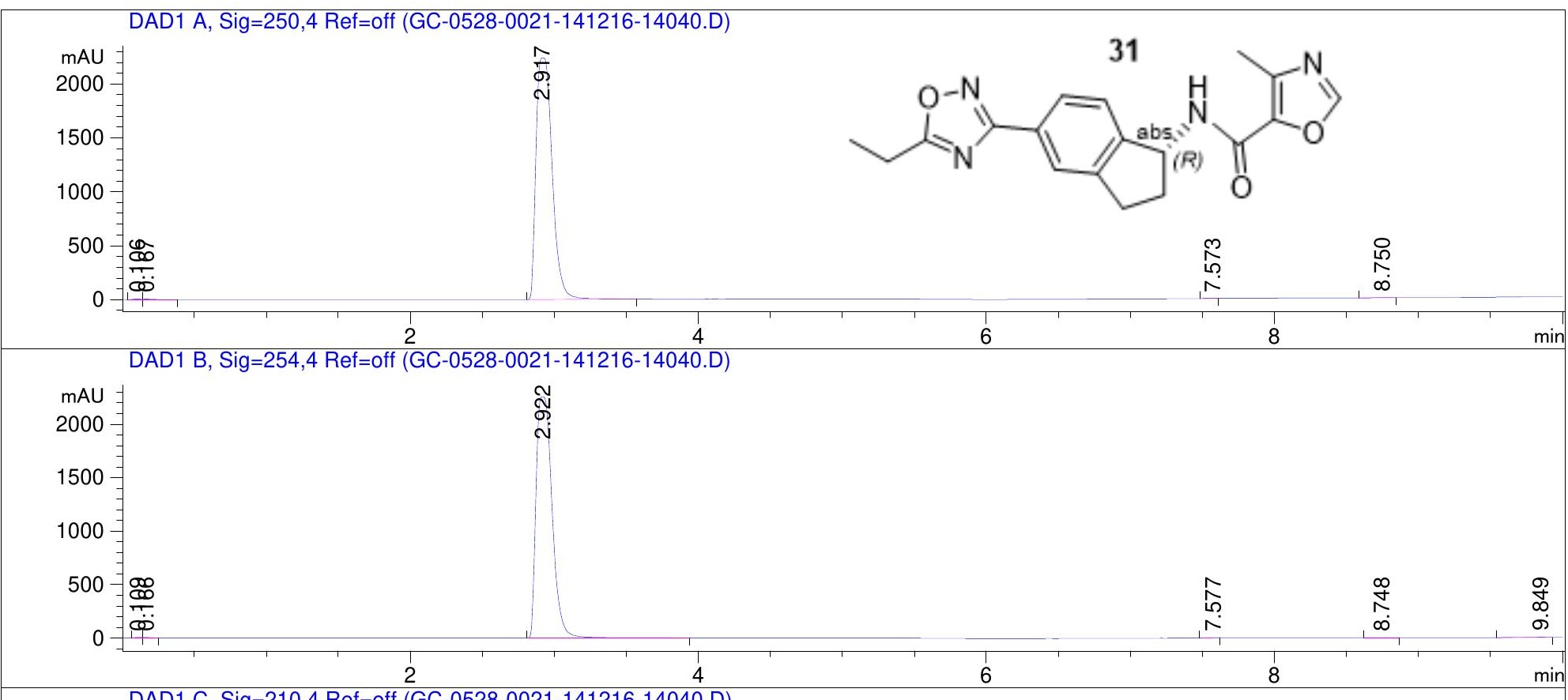

DAD1 C, Sig=210,4 Ref=off (GC-0528-0021-141216-14040.D)

Inj Volume : $5.000 \mu l$
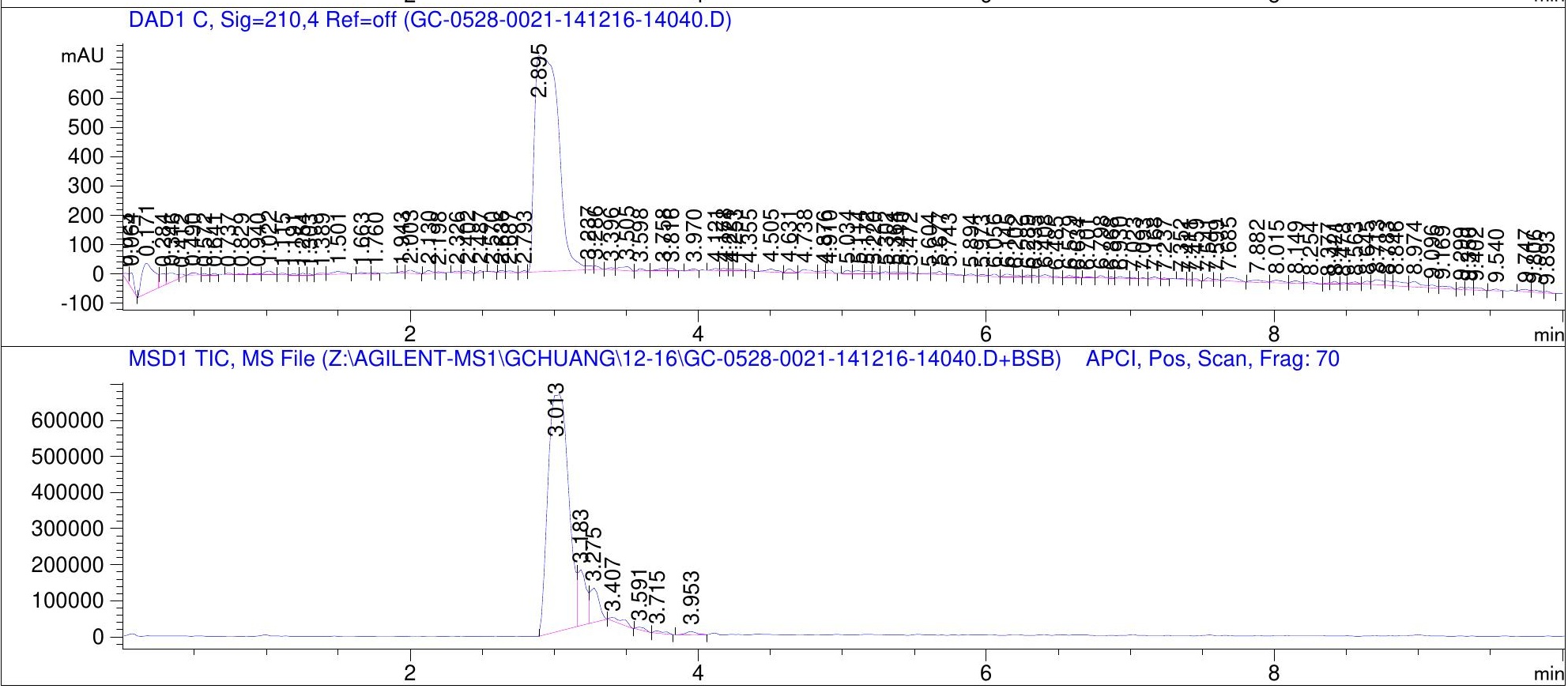
Data File Z: \AGILENT-MS1\GCHUANG \12-16\GC-0528-0021-141216-14040.D

Sample Name: gc-0528-002

MS Signal: MSD1 TIC, MS File, APCI, Pos, Scan, Frag: 70

Subtracted background from 0.000 to $3.000 \mathrm{~min}$.

Spectra averaged over upper half of peaks.

Noise Cutoff: 1000 counts.

Reportable Ion Abundance: > 10\%.

LC Signal: DAD1 A, Sig=250,4 Ref=off

Peak matching window: $0.1 \mathrm{~min}$

\begin{tabular}{|c|c|c|c|c|c|}
\hline \multirow{2}{*}{$\begin{array}{c}\text { Retention } \\
\text { Time (LC) } \\
0.106\end{array}$} & \multicolumn{3}{|c|}{ Retention } & \multicolumn{2}{|c|}{$\begin{array}{l}\text { Mol. Weight } \\
\text { or Ion }\end{array}$} \\
\hline & 16 & - & - & & \\
\hline 0.167 & 17 & - & - & & \\
\hline 2.917 & 17282 & 3.013 & 6481986 & $\begin{array}{l}350.10 \\
349.10\end{array}$ & $\begin{array}{l}I \\
I\end{array}$ \\
\hline- & - & 3.183 & 622413 & $\begin{array}{l}350.10 \\
349.10\end{array}$ & $\begin{array}{l}I \\
I\end{array}$ \\
\hline- & - & 3.275 & 422861 & $\begin{array}{l}350.10 \\
349.10\end{array}$ & $\begin{array}{l}I \\
I\end{array}$ \\
\hline - & - & 3.407 & 95760 & $\begin{array}{l}350.10 \\
349.10 \\
278.05\end{array}$ & $\begin{array}{l}\text { I } \\
\text { I } \\
\text { I }\end{array}$ \\
\hline - & - & 3.591 & 31962 & $\begin{array}{l}350.10 \\
349.10 \\
278.10\end{array}$ & $\begin{array}{l}I \\
I \\
I\end{array}$ \\
\hline - & - & 3.715 & 36475 & $\begin{array}{l}350.10 \\
349.10 \\
295.05 \\
278.10\end{array}$ & $\begin{array}{l}\text { I } \\
\text { I } \\
\text { I } \\
\text { I }\end{array}$ \\
\hline- & - & 3.953 & 46365 & $\begin{array}{l}349.10 \\
295.10 \\
278.10\end{array}$ & $\begin{array}{l}I \\
I \\
I\end{array}$ \\
\hline
\end{tabular}

7.573

8

8.750

19

*MSD1 SPC, time=2.968:3.095 of Z:IAGILENT-MS1/gchuangl12-16\GC-0528-0021-141216-14040.D+BSB APCI, Pos, Scan, Frag: 7
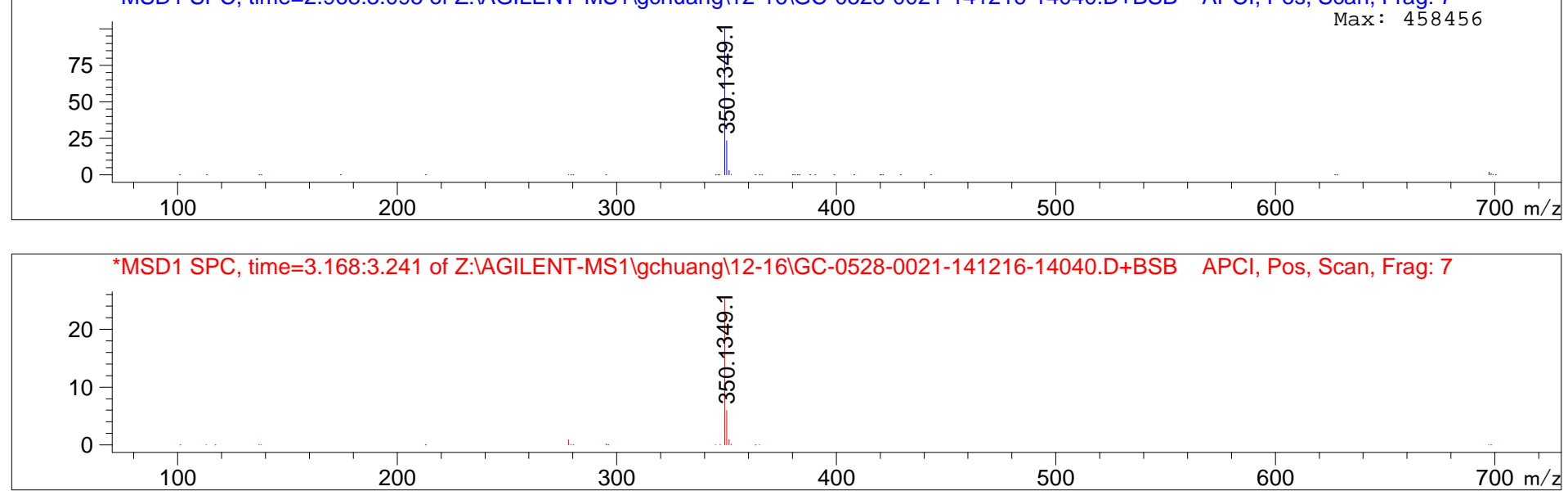
Data File Z: \AGILENT-MS1\GCHUANG \12-16\GC-0528-0021-141216-14040.D

Sample Name: gc-0528-002
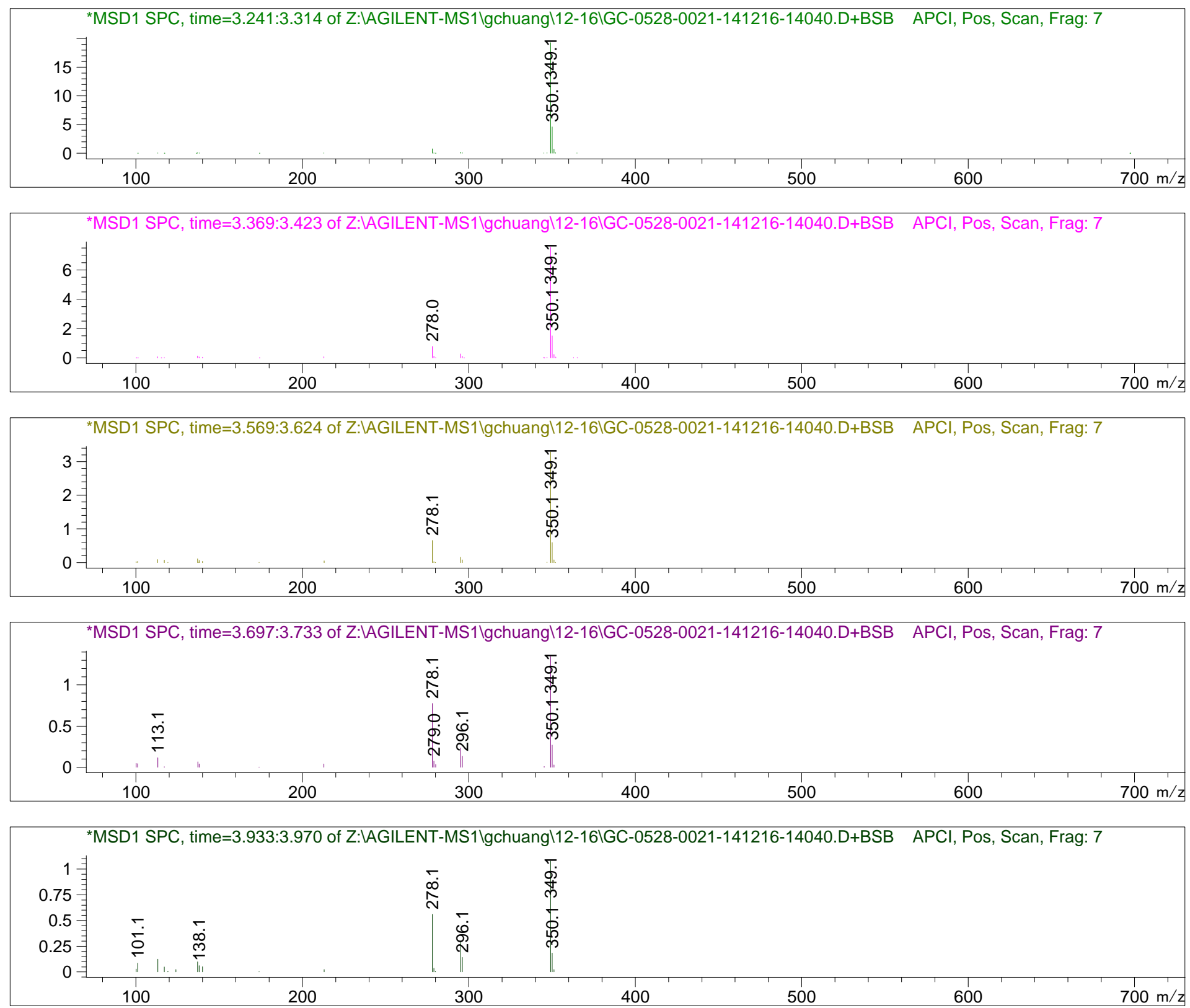
Data File D: \MassHunter\gchuang \2017-04-28\10M_POS_gC-0646-001_2549.D

Sample Name: gc-0646-001

Walkup method: '10MIN POS'
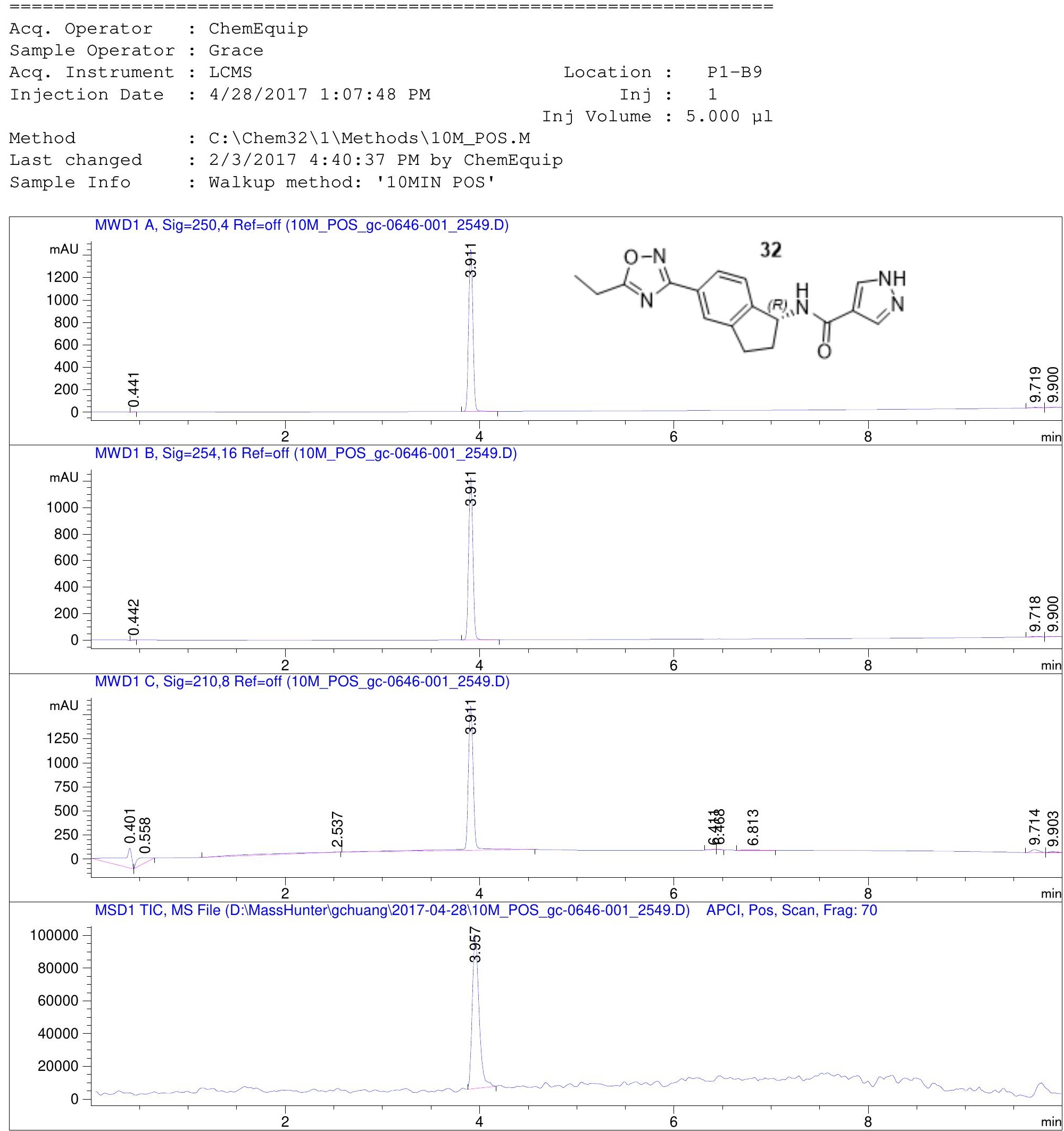
Data File D: \MassHunter\gchuang \2017-04-28\10M_POS_gC-0646-001_2549.D

Sample Name: gc-0646-001

MS Signal: MSD1 TIC, MS File, APCI, Pos, Scan, Frag: 70 Spectra averaged over upper half of peaks.

Noise Cutoff: 1000 counts.

Reportable Ion Abundance: > 10\%.

Retention

Time (MS)

3.957

454323
Mol. Weight

or Ion

$325.10 \mathrm{I}$

$324.10 \mathrm{I}$

*MSD1 SPC, time=3.934:3.988 of D:IMassHunterlgchuangl2017-04-28110M_POS_gc-0646-001_2549.D APCI, Pos, Scan, Frag: 70

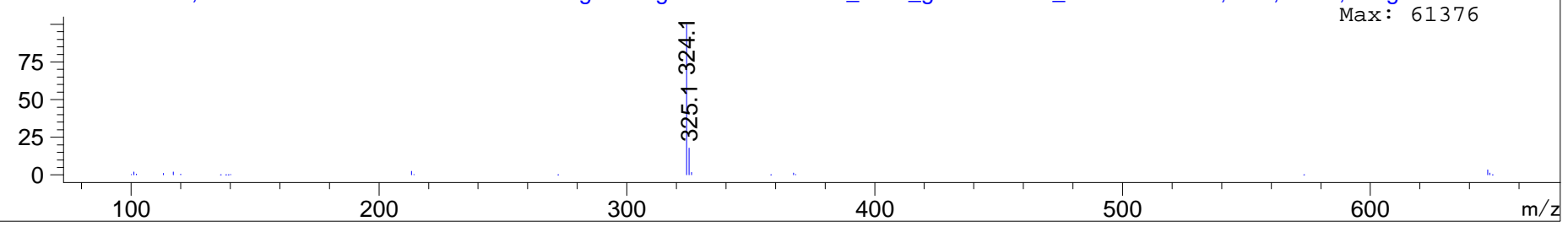




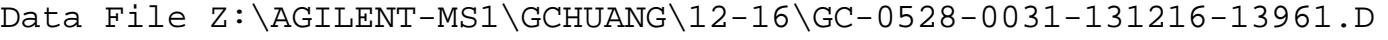

Sample Name: gc-0528-003

Easy-Access Method: '10M_POS'

$========================$
Acq. Operator : chemequip

Sample Operator : chemequip

Acq. Instrument : AGILENTMS1

Injection Date : 12/13/2016 1:18:45 PM

Location : Vial 40

Inj : 1

Method : C: \CHEM32\2\METHODS \10M_POS.M

Last changed : 12/13/2016 1:17:38 PM by chemequip

(modified after loading)

Sample Info : Easy-Access Method: '10M_PoS'
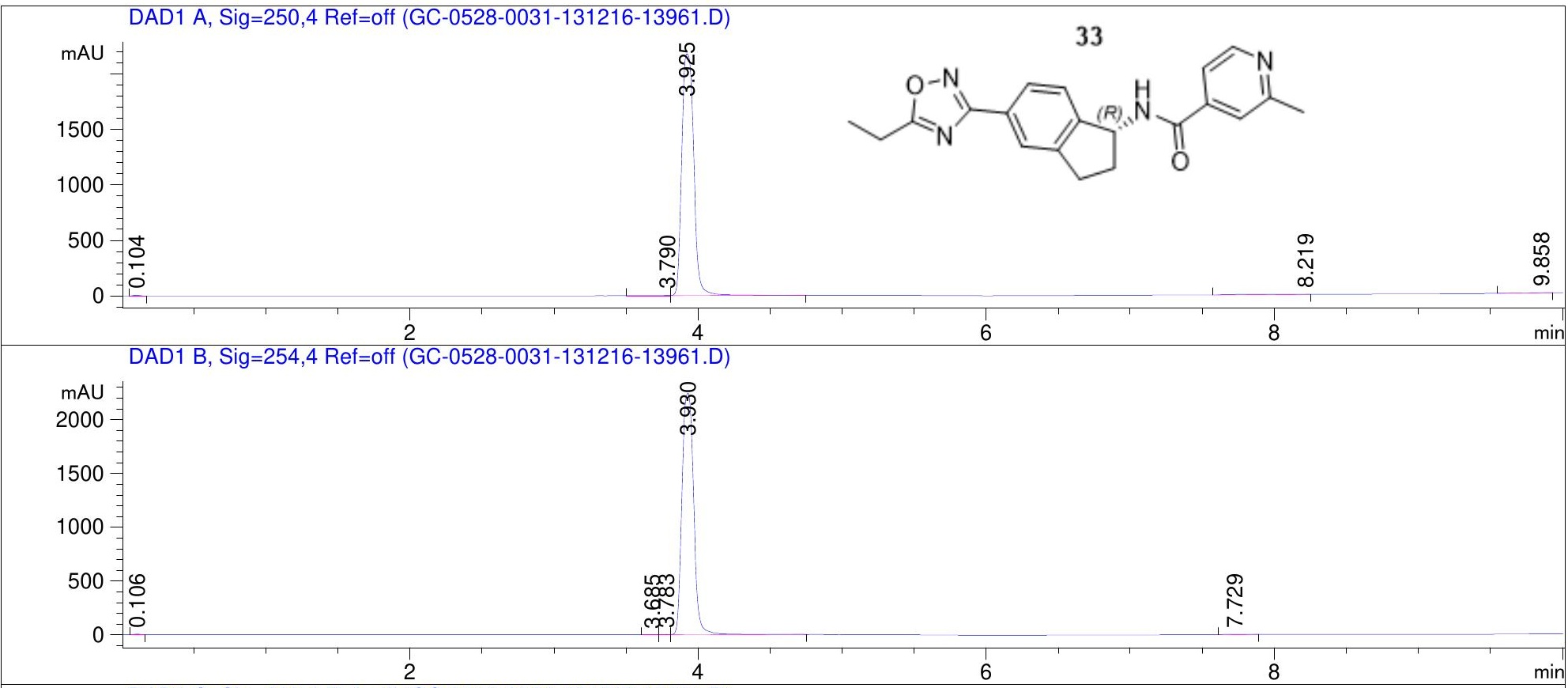

DAD1 C, Sig=210,4 Ref=off (GC-0528-0031-131216-13961.D)

Inj Volume : $5.000 \mu l$
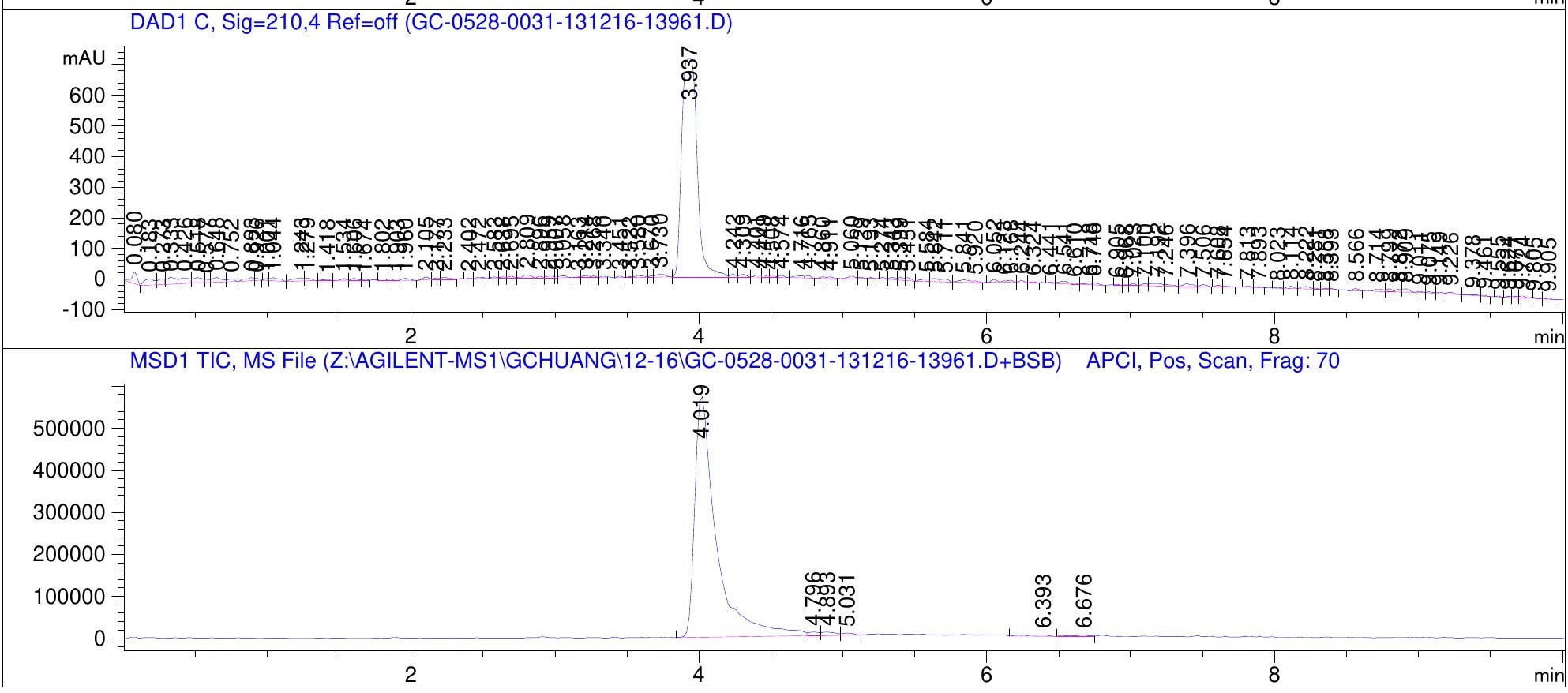


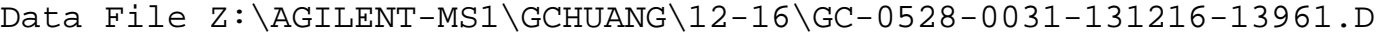

Sample Name: gc-0528-003

MS Signal: MSD1 TIC, MS File, APCI, Pos, Scan, Frag: 70

Subtracted background from 0.000 to $3.000 \mathrm{~min}$.

Spectra averaged over upper half of peaks.

Noise Cutoff: 1000 counts.

Reportable Ion Abundance: > 10\%.

LC Signal: DAD1 A, Sig=250,4 Ref=off

Peak matching window: $0.1 \mathrm{~min}$

\begin{tabular}{|c|c|c|c|c|c|}
\hline Retention & & Retention & & Mol. Wei & $g h$ \\
\hline Time (LC) & LC Area & Time (MS) & MS Area & or Ion & \\
\hline 0.104 & 15 & - & - & & \\
\hline 3.790 & 16 & - & - & & \\
\hline 3.925 & 12566 & 4.019 & 5957233 & 340.10 & I \\
\hline & & & & 339.10 & 1 \\
\hline - & - & 4.796 & 42777 & 340.10 & I \\
\hline & & & & 339.10 & I \\
\hline - & - & 4.893 & 56525 & 340.05 & I \\
\hline & & & & 339.10 & I \\
\hline - & - & 5.031 & 30138 & 340.10 & I \\
\hline & & & & 339.10 & I \\
\hline - & - & 6.393 & 29104 & 339.10 & I \\
\hline & & & & 113.10 & I \\
\hline- & - & 6.676 & 27680 & 339.00 & I \\
\hline & & & & 113.10 & I \\
\hline & & & & 101.00 & I \\
\hline
\end{tabular}
8.219
78
9.858
18
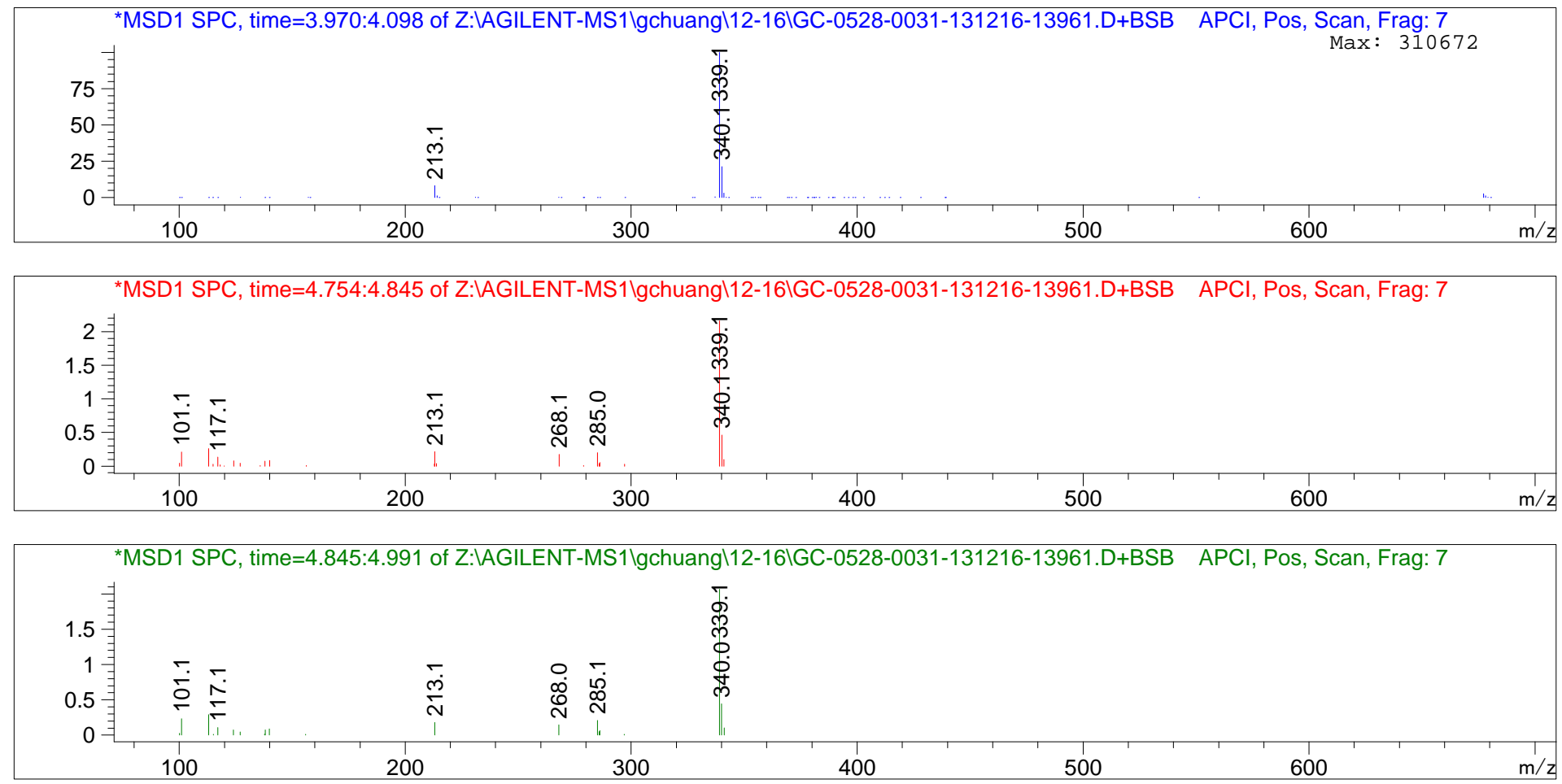
Data File Z: \AGILENT-MS1 \GCHUANG \12-16\GC-0528-0031-131216-13961.D

Sample Name: gc-0528-003
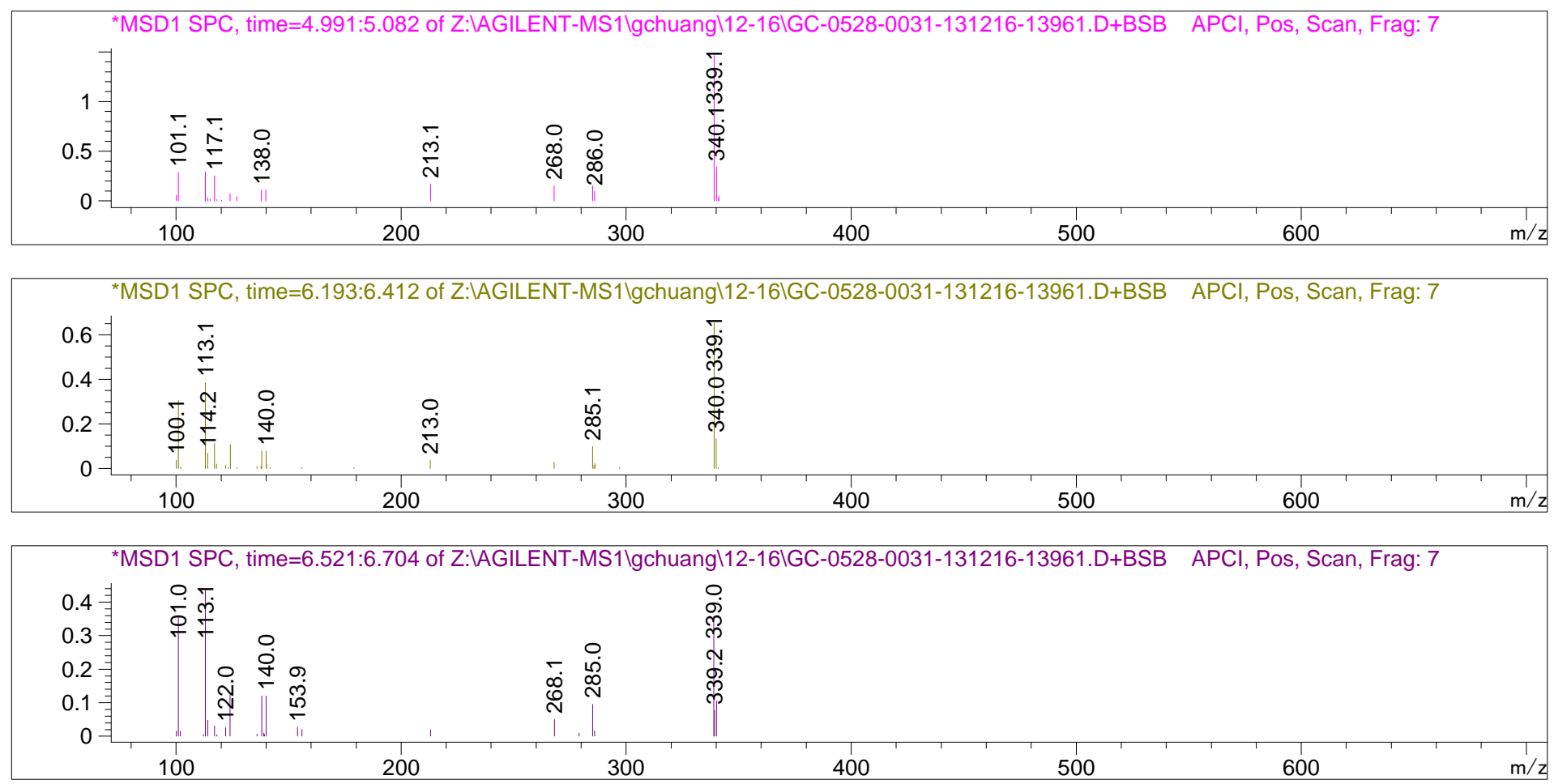
Data File D: \MassHunter\wwang \2018-02-16\3M_POS_ww-0433-1000_2485.D

Sample Name: ww-0433-1000

Walkup method: '3MIN POS'

\begin{tabular}{|c|c|c|c|}
\hline $\begin{array}{l}\text { Acq. Operator } \\
\text { Sample Operator }\end{array}$ & $\begin{array}{l}: \text { chemequip } \\
: \text { Wenyue }\end{array}$ & & \\
\hline Acq. Instrument & : LCMS & Location & $\mathrm{P} 1-\mathrm{E} 8$ \\
\hline Injection Date & : 2/16/2018 4:22:24 PM & $\begin{array}{r}\operatorname{Inj} \\
\text { Inj Volume }\end{array}$ & $\begin{array}{l}: \quad 1 \\
: \quad 5.000 \mu 1\end{array}$ \\
\hline Method & $: C: \backslash$ Chem32 $\backslash 1 \backslash$ Methods $\backslash 3$ M_POS.M & & \\
\hline Last changed & : 2/6/2017 3:07:51 PM by SYSTEM & & \\
\hline Sample Info & : Walkup method: '3MIN POS' & & \\
\hline
\end{tabular}
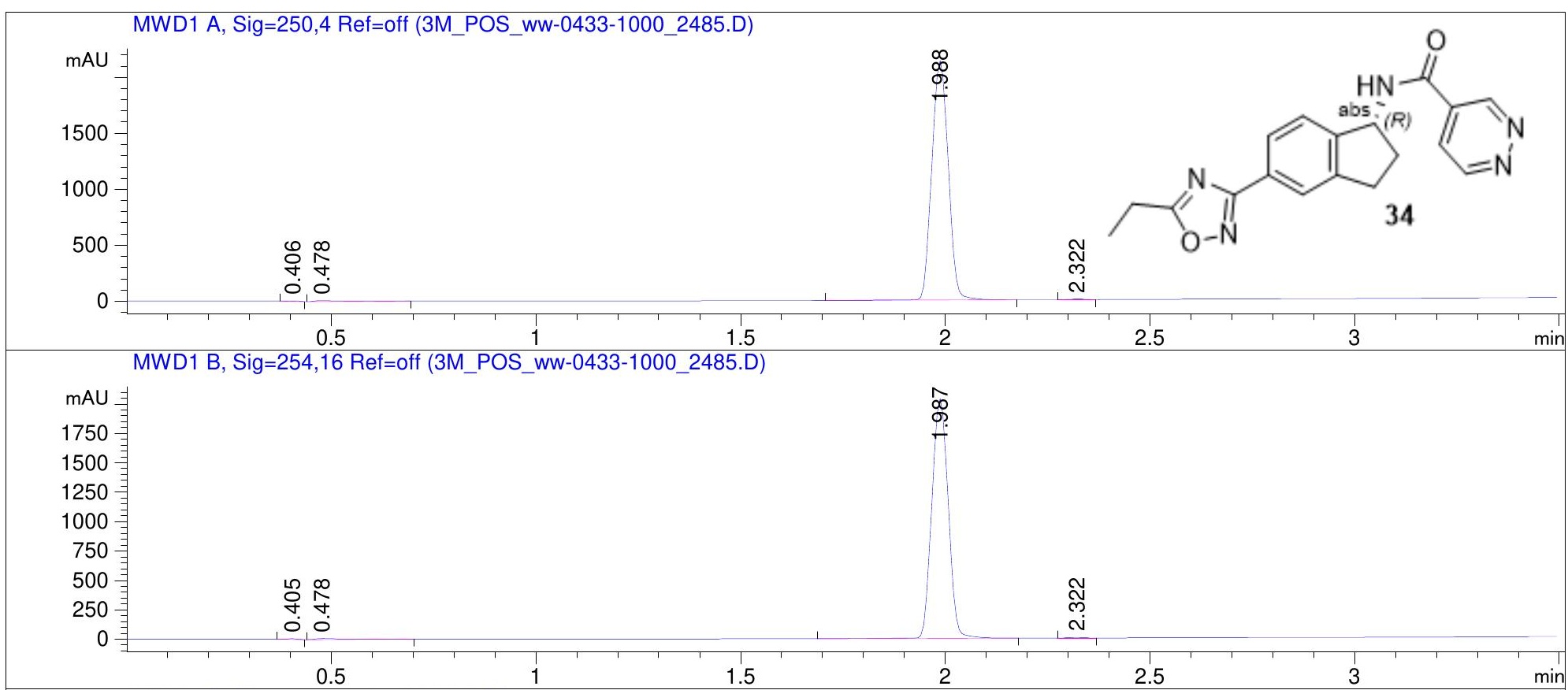

MWD1 C, Sig=210,8 Ref=off (3M_POS_ww-0433-1000_2485.D)

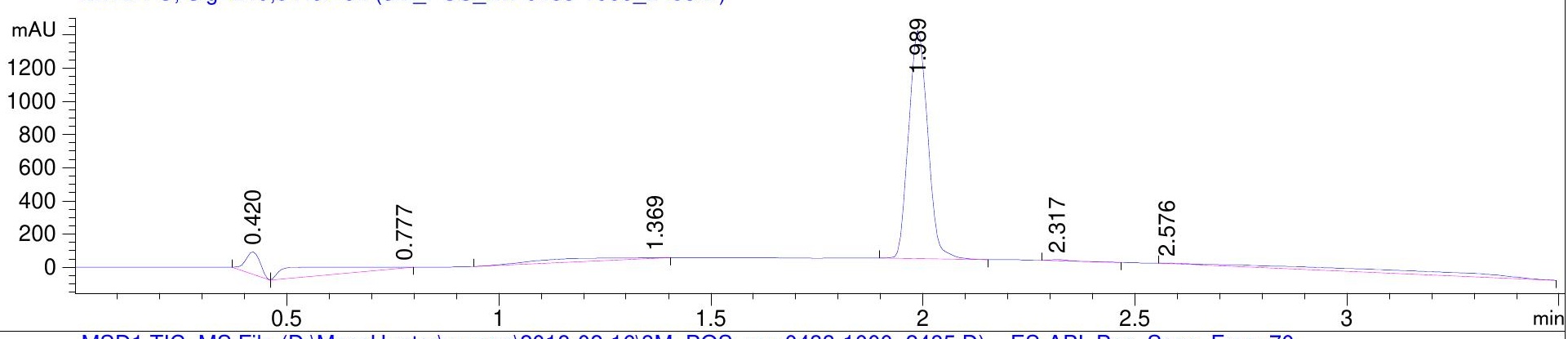

MSD1 TIC, MS File (D:IMassHunterlwwangl2018-02-1613M_POS_ww-0433-1000_2485.D) ES-API, Pos, Scan, Frag: 70

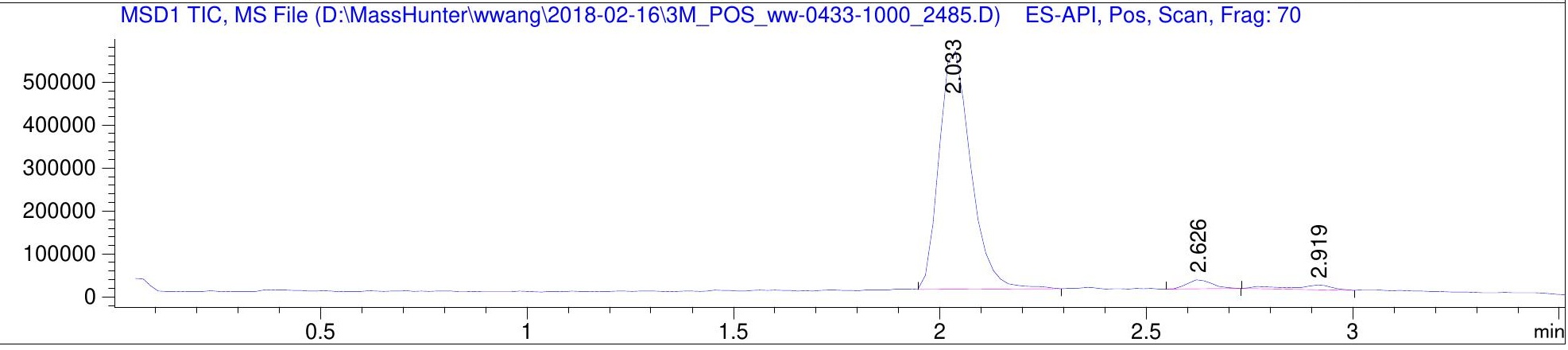


Data File D: \MassHunter\wwang \2018-02-16\3M_POS_ww-0433-1000_2485.D

Sample Name: ww-0433-1000

MS Signal: MSD1 TIC, MS File, ES-API, Pos, Scan, Frag: 70

Spectra averaged over upper half of peaks.

Noise Cutoff: 1000 counts.

Reportable Ion Abundance: > 10\%.

Retention

Time (MS)

2.033

2.626

2.919
MS Area

2962806

87395

73272
Mol. Weight

or Ion

$337.10 \mathrm{I}$

$336.10 \mathrm{I}$

$241.95 \mathrm{I}$

$241.10 \mathrm{I}$

$145.00 \mathrm{I}$

$130.10 \mathrm{I}$

$241.00 \mathrm{I}$

$145.00 \mathrm{I}$

$130.15 \mathrm{I}$

$120.05 \mathrm{I}$

$102.20 \mathrm{I}$
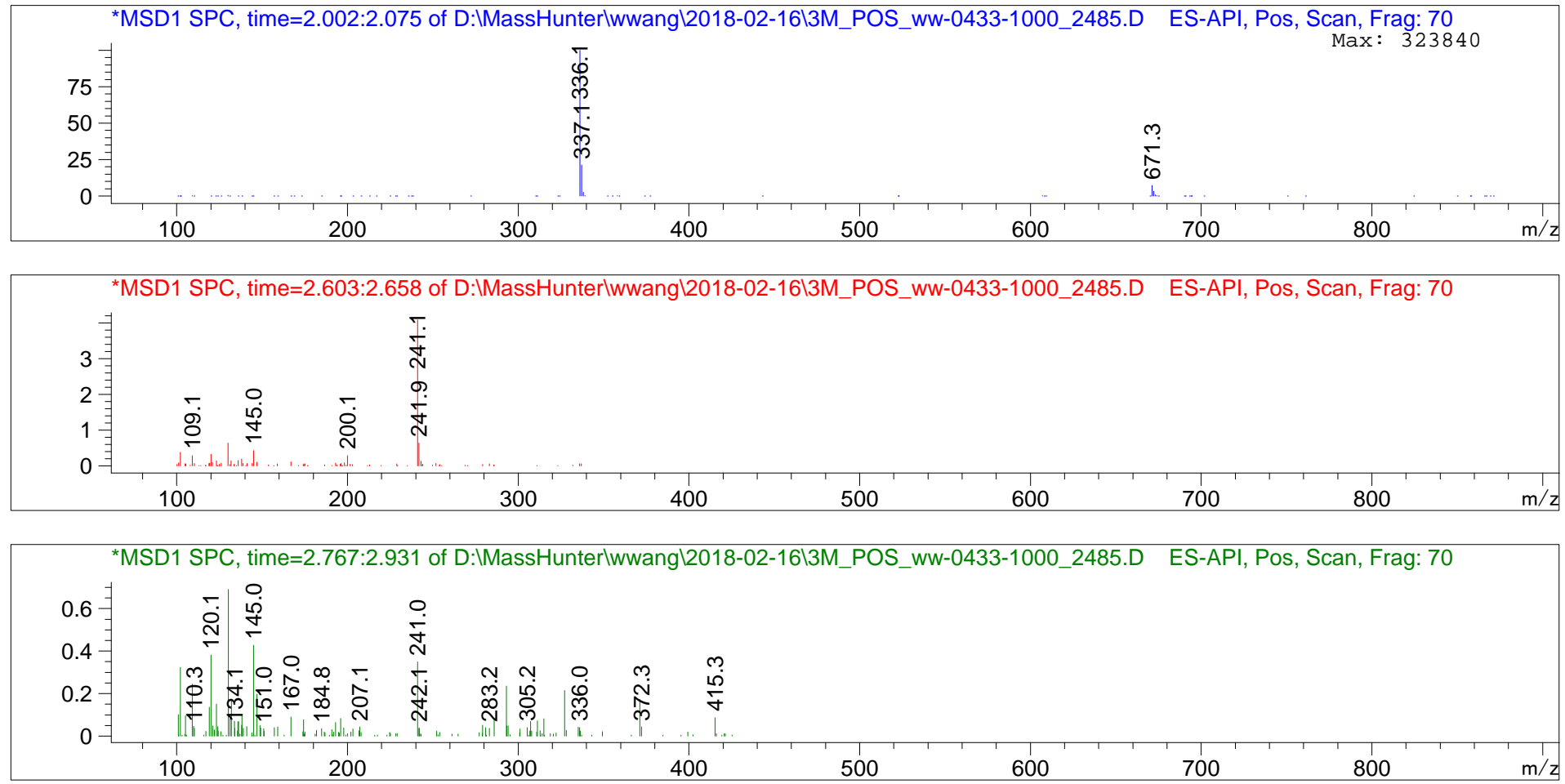
Data File D: \MassHunter\wwang \2018-02-16\3M_POS_ww-0433-900_2484.D

Sample Name: ww-0433-900

Walkup method: '3MIN POS'

\begin{tabular}{|c|c|c|c|}
\hline $\begin{array}{l}\text { Acq. Operator } \\
\text { Sample Operator }\end{array}$ & $\begin{array}{l}\text { : chemequip } \\
: \text { Wenyue }\end{array}$ & & \\
\hline Acq. Instrument & : LCMS & Location & $\mathrm{P} 1-\mathrm{E} 7$ \\
\hline Injection Date & : 2/16/2018 4:09:19 PM & $\begin{array}{r}\operatorname{Inj} \\
\text { Inj Volume }\end{array}$ & $\begin{array}{l}: \quad 1 \\
: \quad 5.000 \mu 1\end{array}$ \\
\hline Method & : C: $\backslash$ Chem32 $\backslash 1 \backslash$ Methods $\backslash 3$ M_POS.M & & \\
\hline Last changed & : 2/6/2017 3:07:51 PM by SYSTEM & & \\
\hline Sample Info & : Walkup method: '3MIN POS' & & \\
\hline
\end{tabular}

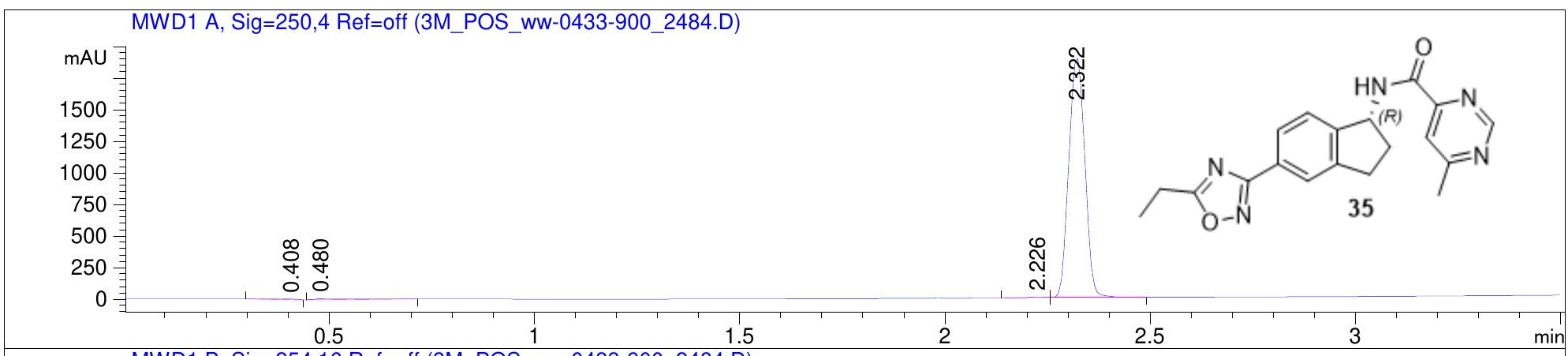

MWD1 B, Sig=254,16 Ref=off (3M_POS_ww-0433-900_2484.D)

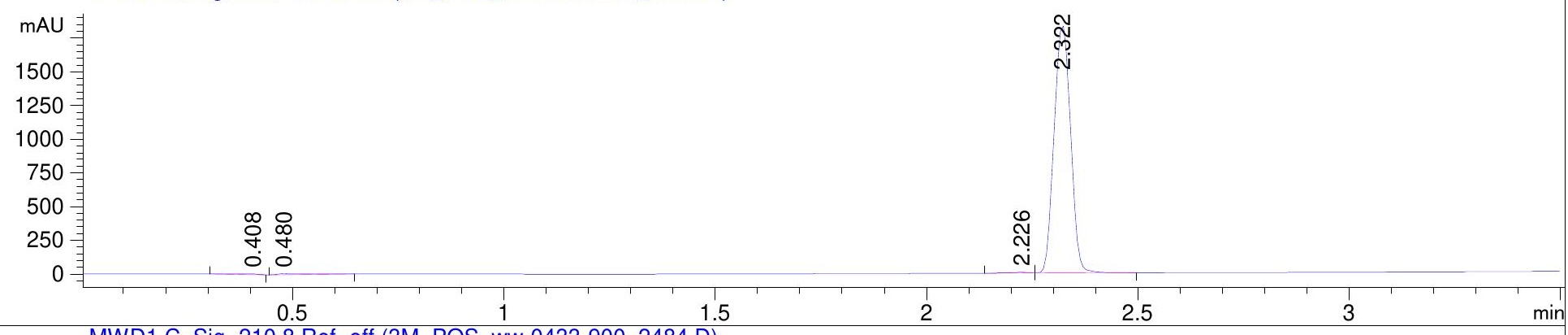

MWD1 C, Sig=210,8 Ref=off (3M_POS_ww-0433-900_2484.D)

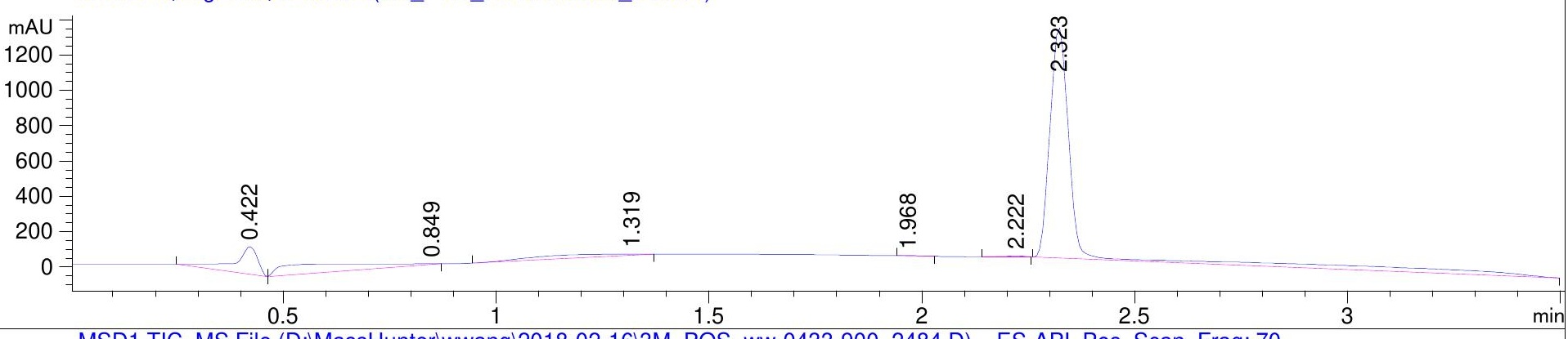

MSD1 TIC, MS File (D:MassHunterlwwangl2018-02-1613M POS ww-0433-900 2484.D) ES-API, Pos, Scan, Frag: 70

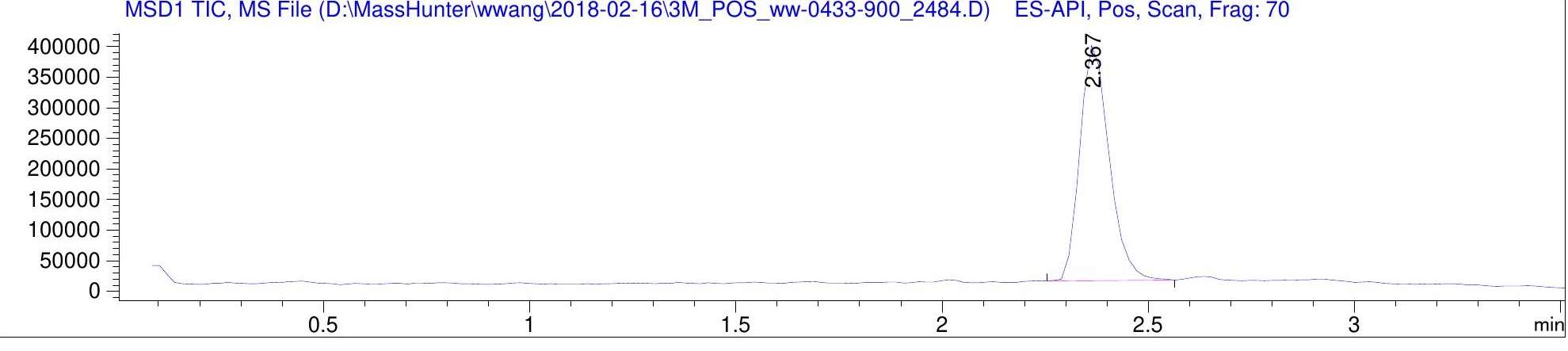


Data File D: \MassHunter\wwang \2018-02-16\3M_POS_ww-0433-900_2484.D

Sample Name: ww-0433-900

MS Signal: MSD1 TIC, MS File, ES-API, Pos, Scan, Frag: 70

Spectra averaged over upper half of peaks.

Noise Cutoff: 1000 counts.

Reportable Ion Abundance: > 10\%.

Retention

Time (MS)

2.367
MS Area

1896493
Mol. Weight

or Ion

$$
\begin{array}{ll}
699.30 & I \\
351.10 & I \\
350.10 & I \\
214.10 & I \\
213.10 & I \\
138.10 & I
\end{array}
$$

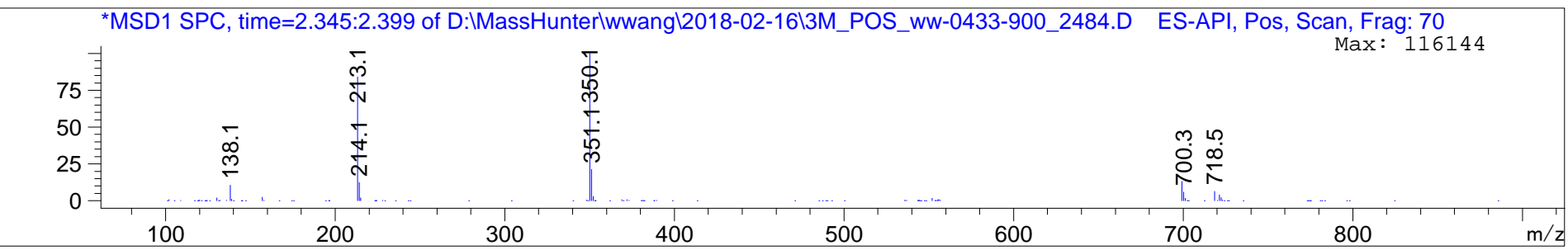




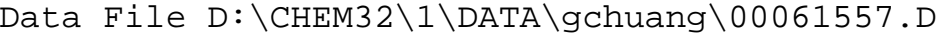

Sample Name: gc-0362-001
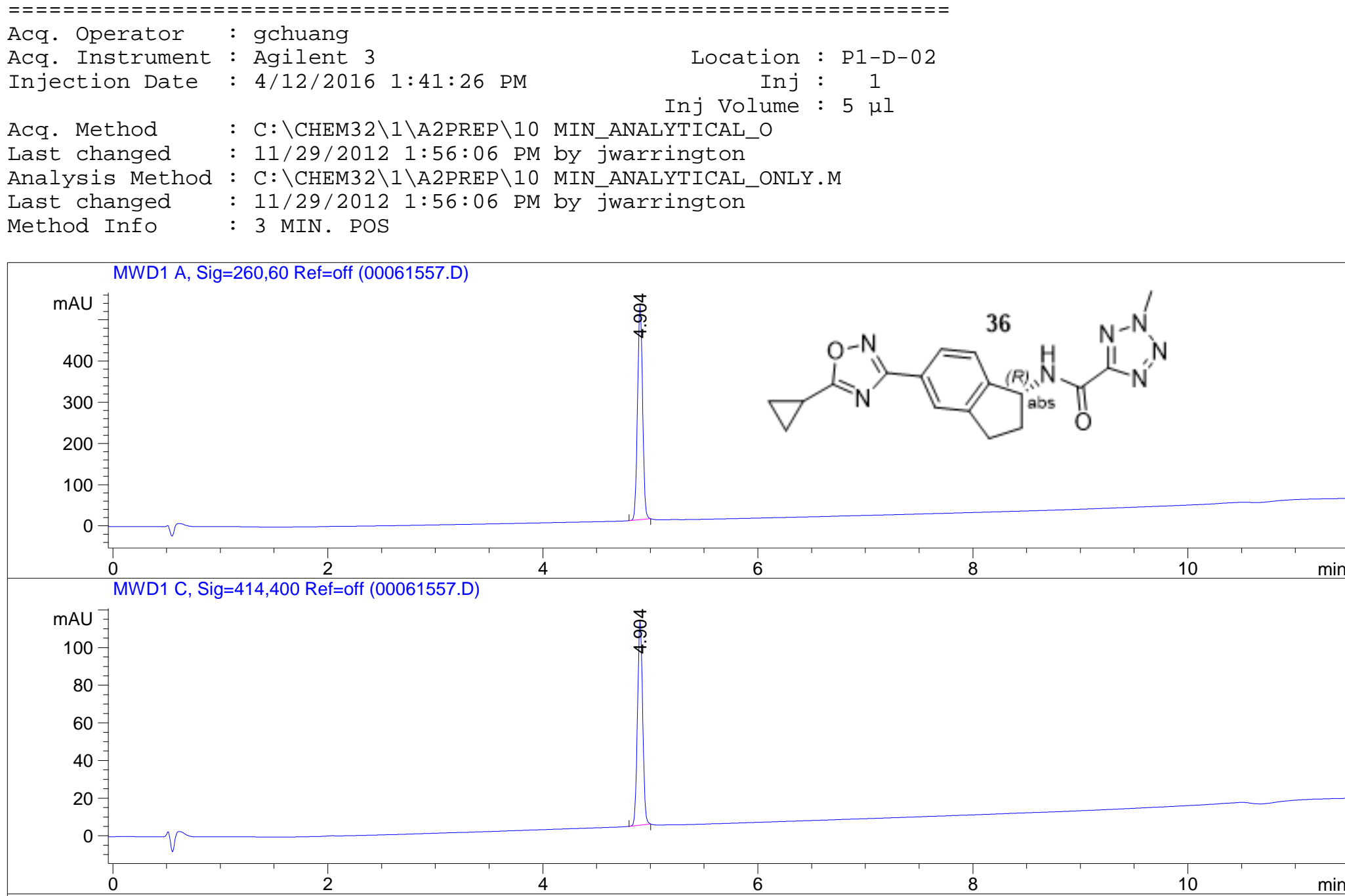
MWD1 B, Sig=254,16 Ref=360,100 (00061557.D)

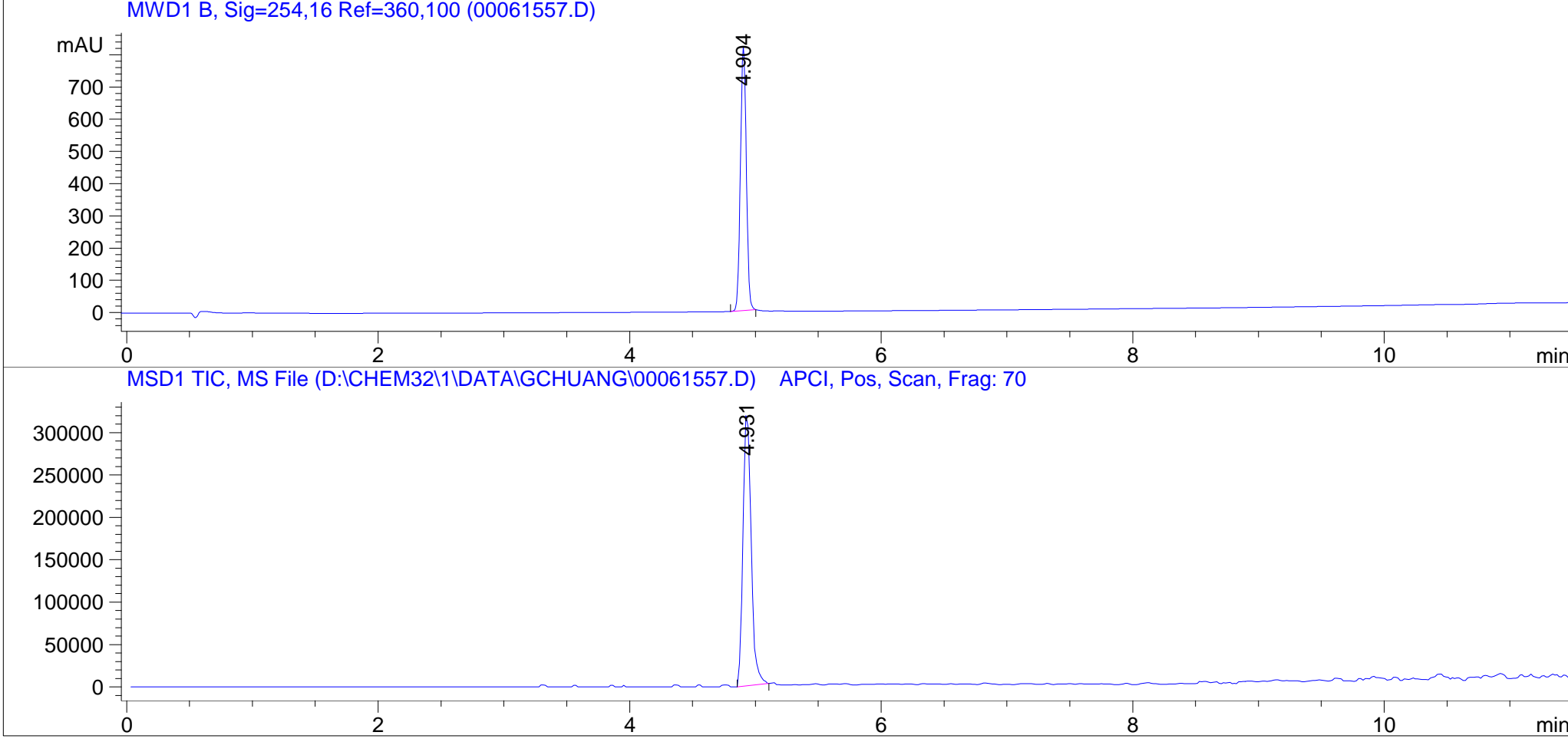




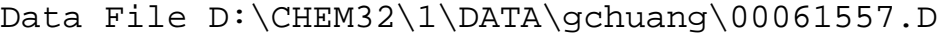

Sample Name: gc-0362-001

MS Signal: MSD1 TIC, MS File, APCI, Pos, Scan, Frag: 70 Spectra averaged over upper half of peaks. Noise Cutoff: 1000 counts.

Reportable Ion Abundance: > 10\%.

Retention

Time (MS)

4.931
MS Area

1451166
Mol. Weight

or Ion

353.10
352.20
352.10
225.10

$352.20 I$

$225.10 \mathrm{I}$

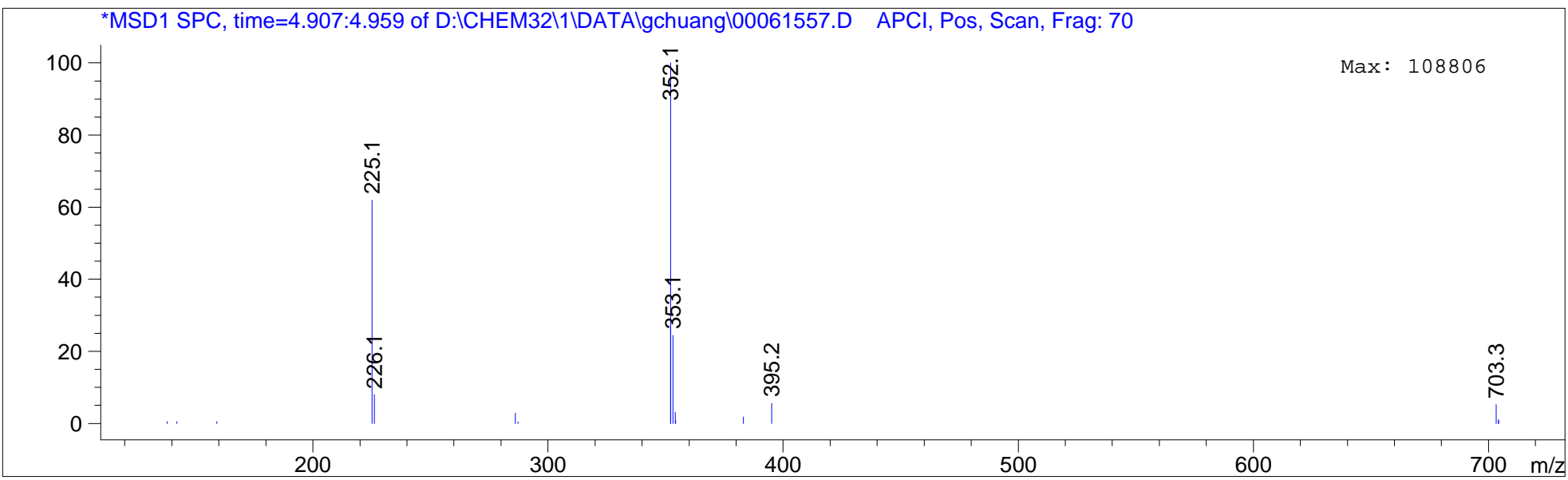




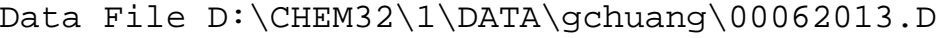

Sample Name: gc-0383-001
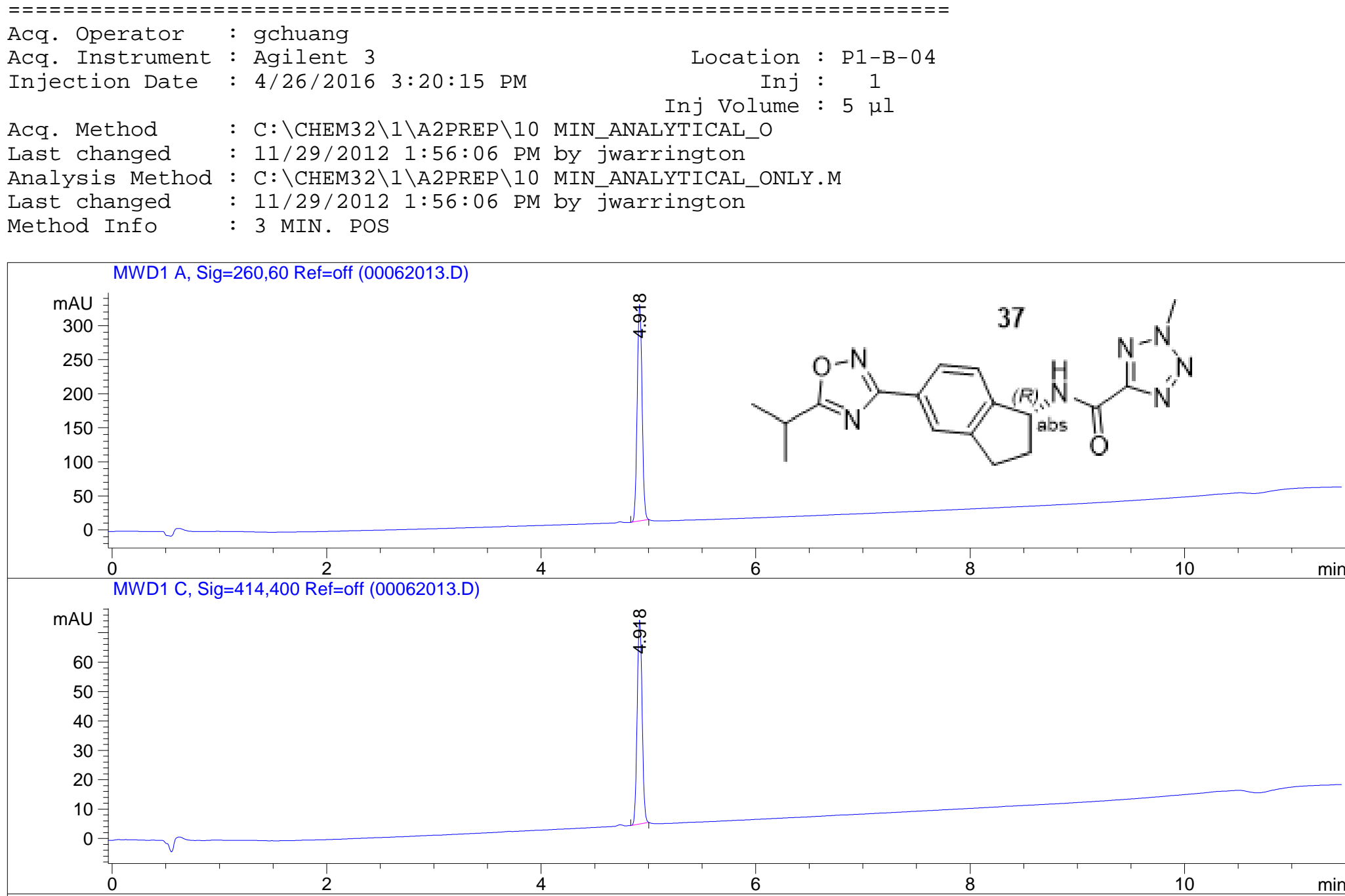
MWD1 B, Sig=254,16 Ref=360,100 (00062013.D)

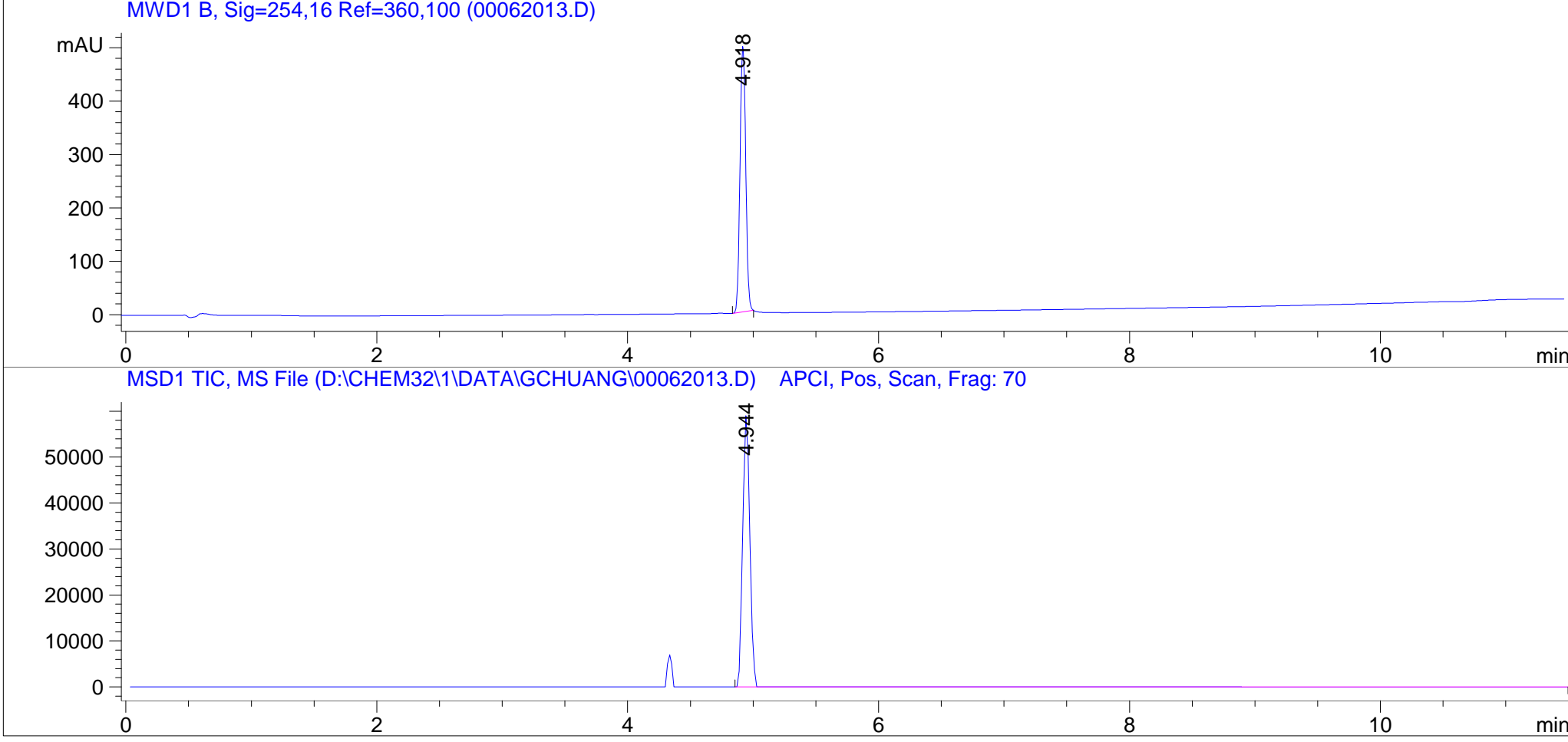


Data File Z:\AGILENT-MS1\GCHUANG\12-16\GC-0528-0051-141216-14023.D

Sample Name: gc-0528-005

Easy-Access Method: '10M_POS'

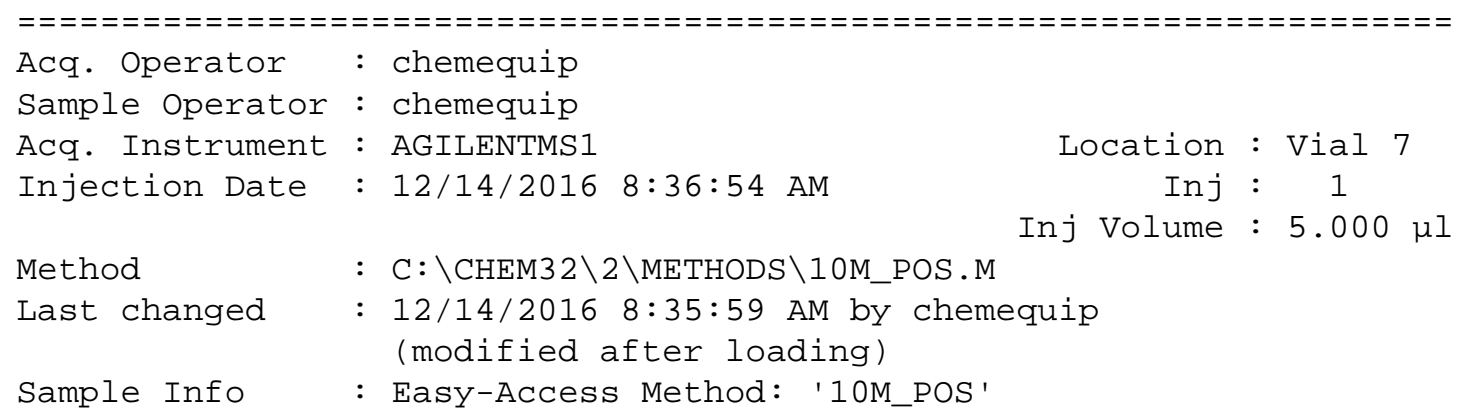
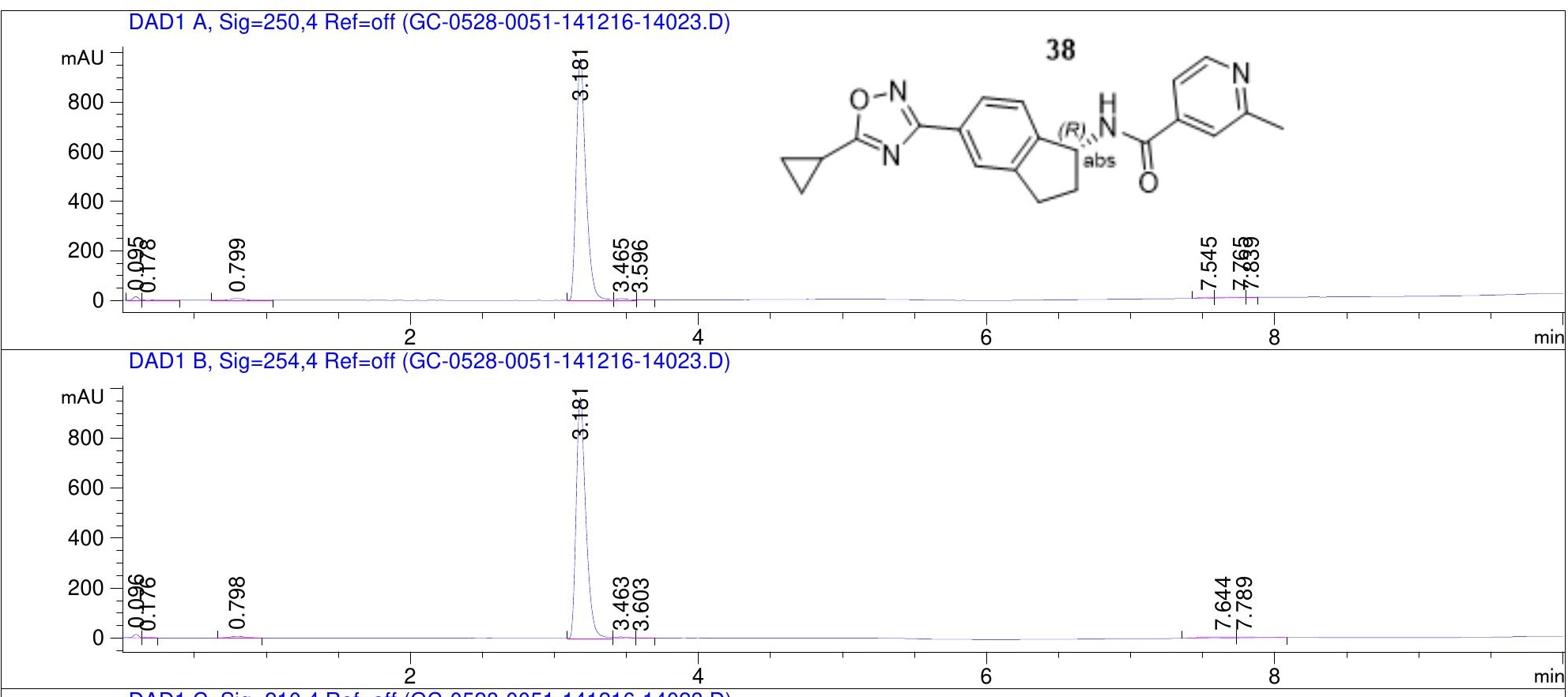

DAD1 C, Sig=210,4 Ref=off (GC-0528-0051-141216-14023.D)
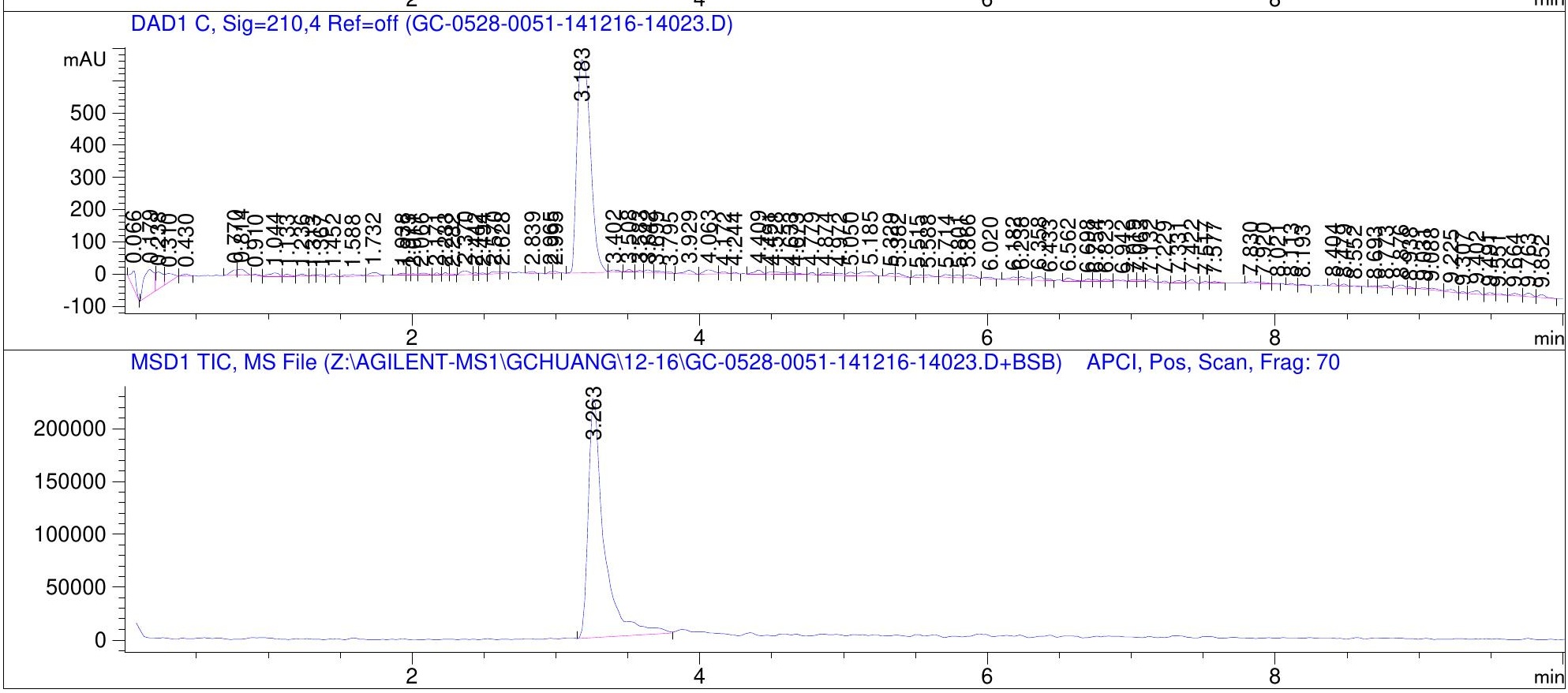
Data File Z: \AGILENT-MS1\GCHUANG \12-16\GC-0528-0051-141216-14023.D

Sample Name: gc-0528-005

MS Signal: MSD1 TIC, MS File, APCI, Pos, Scan, Frag: 70

Subtracted background from 0.000 to $3.000 \mathrm{~min}$.

Spectra averaged over upper half of peaks.

Noise Cutoff: 1000 counts.

Reportable Ion Abundance: > 10\%.

LC Signal: DAD1 A, Sig=250,4 Ref=off

Peak matching window: $0.1 \mathrm{~min}$

\begin{tabular}{|c|c|c|c|c|}
\hline $\begin{array}{l}\text { Retention } \\
\text { Time (LC) }\end{array}$ & LC Area & $\begin{array}{l}\text { Retention } \\
\text { Time (MS) }\end{array}$ & MS Area & $\begin{array}{l}\text { Mol. Weight } \\
\text { or Ion }\end{array}$ \\
\hline 0.095 & 35 & - & - & \\
\hline 0.178 & 10 & - & - & \\
\hline 0.799 & 52 & - & - & \\
\hline 3.181 & 4508 & 3.263 & 1732001 & $\begin{array}{l}362.10 \mathrm{I} \\
361.10 \mathrm{I}\end{array}$ \\
\hline 3.465 & 32 & - & - & \\
\hline 3.596 & 5 & - & - & \\
\hline 7.545 & 25 & - & - & \\
\hline 7.765 & 33 & - & - & \\
\hline 7.839 & 6 & - & - & \\
\hline
\end{tabular}

${ }^{*}$ MSD1 SPC, time=3.238:3.311 of Z:|AGILENT-MS1।gchuangl12-16|GC-0528-0051-141216-14023.D+BSB APCI, Pos, Scan, Frag: 7

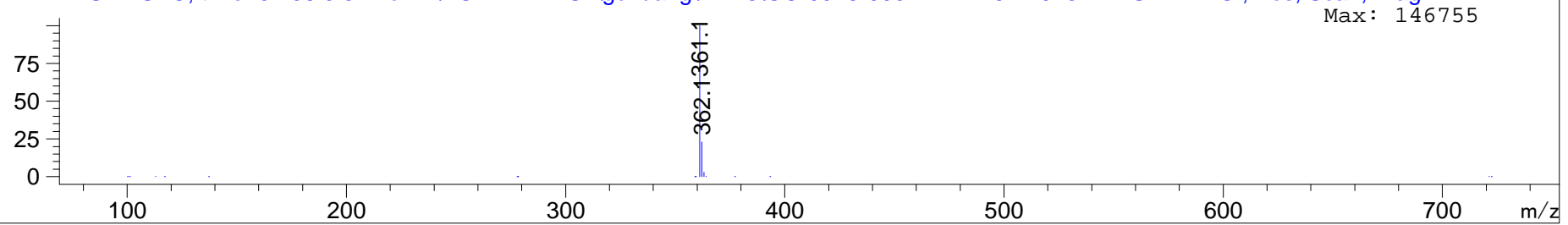


Data File Z:\AGILENT-MS1\GCHUANG\12-16\GC-0528-0081-151216-14097.D

Sample Name: gc-0528-008

Easy-Access Method: '10M_POS'

$========================$
Acq. Operator : chemequip

Sample Operator : chemequip

Acq. Instrument : AGILENTMS1

Injection Date : 12/15/2016 11:20:57 AM

Location : Vial 24

Inj : 1

Method : C : \CHEM32 $2 \backslash$ METHODS $\backslash 10 M \_P O S . M$

Last changed : 12/15/2016 11:20:00 AM by chemequip

(modified after loading)

Sample Info : Easy-Access Method: '10M_PoS'
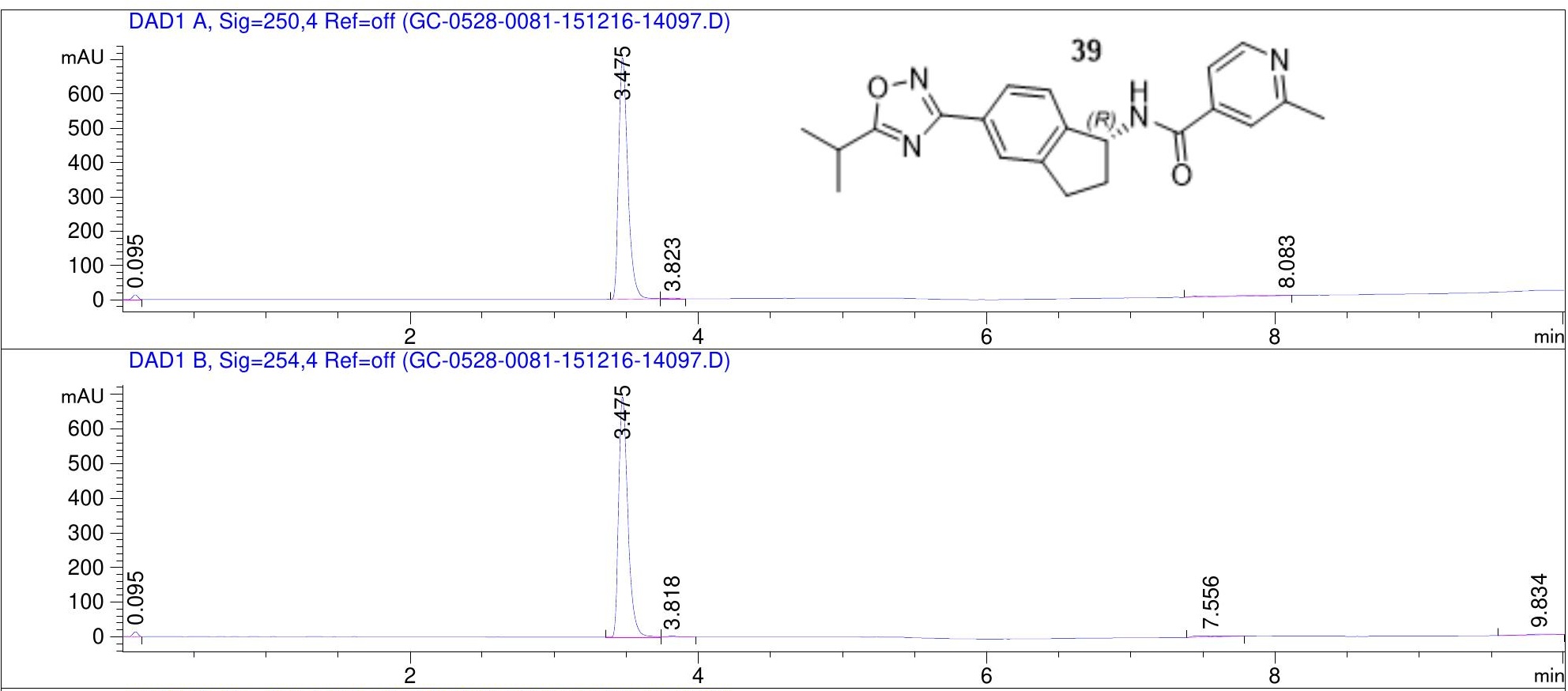

DAD1 C, Sig=210,4 Ref=off (GC-0528-0081-151216-14097.D)
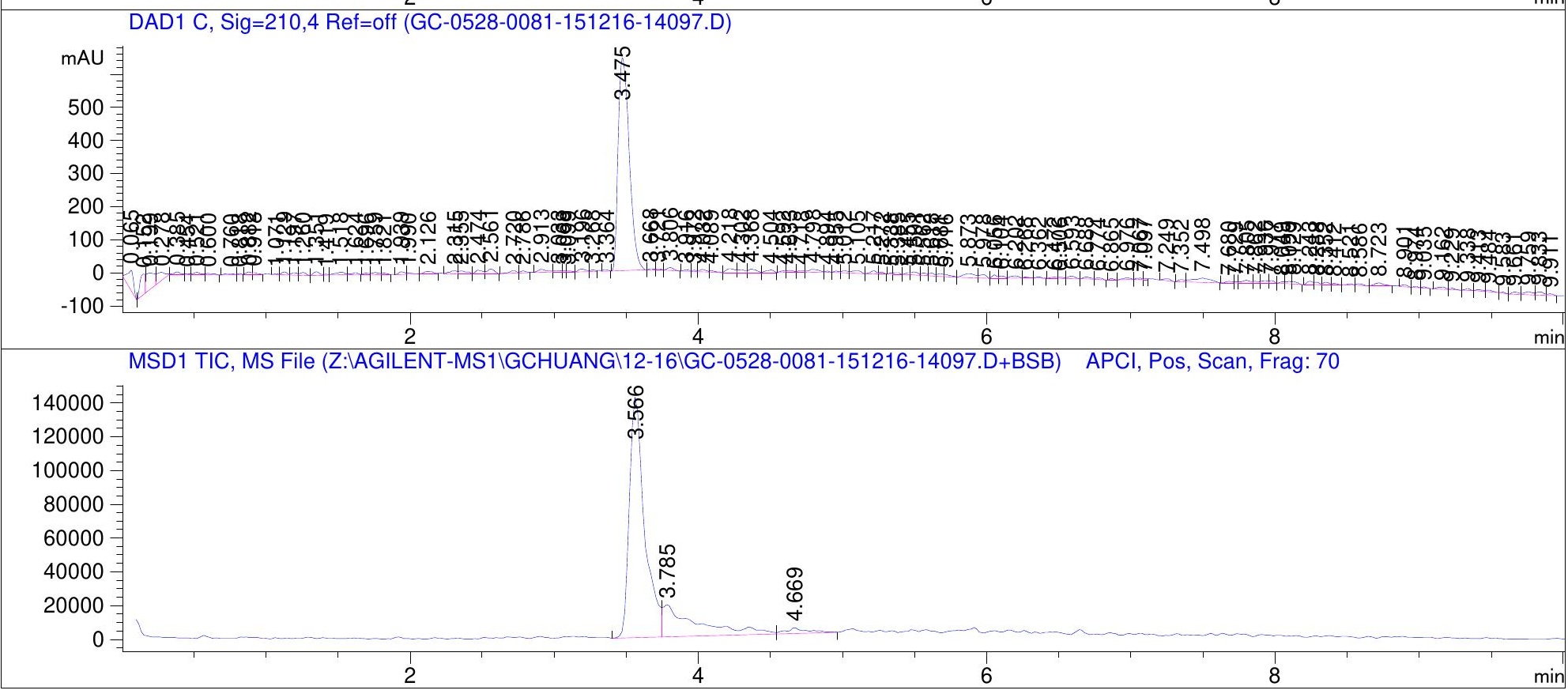
Data File Z:\AGILENT-MS1\GCHUANG\12-16\GC-0528-0081-151216-14097.D

Sample Name: gc-0528-008

MS Signal: MSD1 TIC, MS File, APCI, Pos, Scan, Frag: 70

Subtracted background from 0.000 to $3.000 \mathrm{~min}$.

Spectra averaged over upper half of peaks.

Noise Cutoff: 1000 counts.

Reportable Ion Abundance: > 10\%.

LC Signal: DAD1 A, Sig=250,4 Ref=off

Peak matching window: $0.1 \mathrm{~min}$

\begin{tabular}{|c|c|c|c|c|c|}
\hline \multirow{3}{*}{$\begin{array}{c}\text { Retention } \\
\text { Time (LC) } \\
0.095\end{array}$} & & \multicolumn{2}{|l|}{ Retention } & \multirow{2}{*}{\multicolumn{2}{|c|}{$\begin{array}{l}\text { Mol. Weight } \\
\text { or Ion }\end{array}$}} \\
\hline & LC Area & Time (MS) & MS Area & & \\
\hline & 35 & - & - & & \\
\hline 3.475 & 3102 & 3.566 & 1044294 & 364.10 & I \\
\hline & & & & 363.10 & I \\
\hline 3.823 & 13 & 3.785 & 310852 & 364.10 & I \\
\hline & & & & 363.10 & I \\
\hline- & - & 4.669 & 33988 & 363.10 & I \\
\hline
\end{tabular}

8.083

95
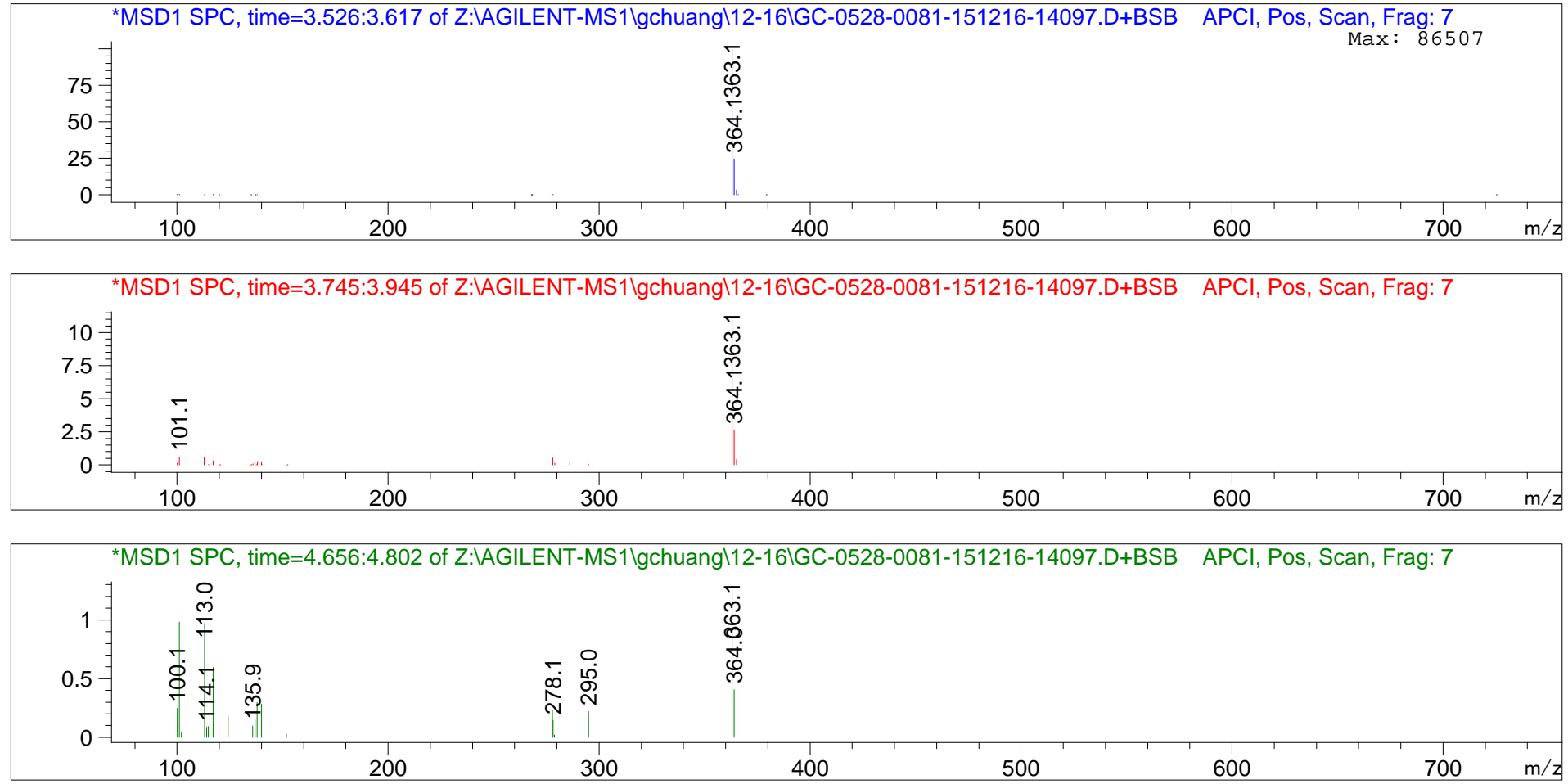